USE OF LOW RATES OF FUNGICIDE

TO SUPPRESS INITIAL WAVES OF PLANT DISEASE

AND TO MANAGE EPIDEMICS

By

CARLOS ALBERTO FORCELINI

A DISSERTATION PRESENTED TO THE GRADUATE SCHOOL OF THE UNIVERSITY OF FLORIDA IN PARTIAL FULFILLMENT OF THE REQUIREMENTS FOR THE DEGREE OF DOCTOR OF PHILOSOPHY

UNIVERSITY OF FLORIDA 
To Eveli, Bruna, and Bernardo, for their love, dreams, and lives. 


\section{ACKNOWLEDGMENTS}

I wish to thank the Universidade de Passo Fundo, which gave permission for this study leave and provided financial support, and the Fundação Capes, which granted the scholarship for this graduate program. My gratitude also goes to the University of Florida, whose human and physical resources provided excellent conditions for my training.

I would like to express my appreciation to Dr. Richard Berger, chairman of my supervisory committee and a very special professor. His guidance and encouragement during this doctoral program will always be remembered. Sincere appreciation is also extended to the other professors who served on my supervisory committee, Dr. James W.

Kimbroug, Dr. Thomas Kucharek, and Dr. Kenneth M. Portier, for their kind support and valuable suggestions throughout this study.

I wish to thank all professors and staff of the Plant Pathology department, especially Terry Davoli and Ron Hoover, for their friendship and technical assistance during this doctoral program.

I gratefully acknowledge the graduate students and all other friends, whose delightful company made our journey a lot more enjoyable. Special recognition goes to the Brazilian students, who have finely represented our country at the University of Florida.

Finally, I thank God, whose power made everything possible. 


\section{TABLE OF CONTENTS}

ACKNOWLEDGMENTS .......................................................................

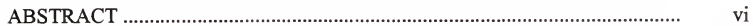

\section{CHAPTERS}

I INTRODUCTION ……..............................................................

II $\quad$ LITERATURE REVIEW .............................................................

Epidemic progress $\ldots \ldots \ldots \ldots \ldots \ldots \ldots \ldots \ldots \ldots \ldots \ldots \ldots \ldots \ldots \ldots \ldots \ldots \ldots, \quad 4$

Epidemic waves ................................................ 5

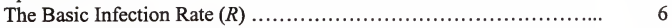

Strategies to Manage Epidemics .................................... 7

Management of Epidemics with Fungicides ......................... 8

Assessment of Fungicidal Effects ................................. 15

Fitting Models and Statistical Analysis of Disease Progress Curves ..... 17

III MANAGEMENT OF EPIDEMICS OF CROWN RUST ON OATS ..... 22

Introduction ................................................................................. 22

Material and Methods ..................................................................... 23

Results ......................................................................................... 34

Discussion .................................................................................. 99

IV MANAGEMENT OF EPIDEMICS OF HELMINTHOSPORIUM LEAF SPOT ON RYE ................................................................... 104

Introduction ..................................................................................... 104

Material and Methods .................................................................... 105

Results ........................................................................................... 107

Discussion .................................................................................. 137 
V MANAGEMENT OF EPIDEMICS OF RUST AND LEAF SPOTS ON PEANUT …................................................................................. 140

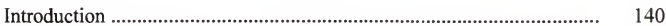

Material and Methods ........................................................................... 144

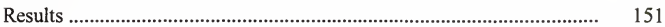

Discussion ........................................................................................... 229

VI MANAGEMENT OF EPIDEMICS OF BLACK SPOT ON ROSES .... 237

Introduction ....................................................................................... 237

Material and Methods .......................................................................... 238

Results ............................................................................................ 241

Discussion ......................................................................................... 274

VII SUMMARY AND CONCLUSIONS .................................................... 278

\section{APPENDICES}

A SCHEDULES OF FUNGICIDAL SPRAYS .......................... 283

B NONLINEAR REGRESSION PARAMETERS .................................... 291

C WEATHER DATA ….......................................................................... 303

D COST-BENEFIT ANALYSIS OF FUNGICIDAL SPRAYS FOR PEANUTS ....................................................................................... 307

LIST OF REFERENCES .................................................................................. 308

BIOGRAPHICAL SKETCH ......................................................................... 319 


\begin{abstract}
Dissertation Presented to the Graduate School of the University of Florida in Partial Fulfillment of the Requirements for the Degree of Doctor of Philosophy
\end{abstract}

\title{
USE OF LOW RATES OF FUNGICIDES \\ TO SUPPRESS INITIAL WAVES OF PLANT DISEASE AND TO MANAGE EPIDEMICS
}

By

\section{Carlos Alberto Forcelini}

December, 1997

Chairman: Dr. Richard D. Berger

Major Department: Plant Pathology

The potential of low rates of protectant fungicides to suppress and delay initial waves of plant disease and to manage epidemics was evaluated in 18 experiments conducted from 1994-1997. Crown rust of oats, the early and late leaf spots of peanuts, peanut rust, black spot of roses, and helminthosporium leaf spot on rye were used as model pathosystems in this research. The average spray intervals were $3.5,6$, and 11 days for the $0.25 \times, 0.5 \times$, and $1 \times$ fungicidal rates, respectively. Compared to standard (1x) spray schedules, the low rates of fungicide delayed epidemic onset $\left(t_{0}\right)$ 2-6 days, decreased the initial disease $\left(y_{0}\right) 40-48 \%$, slowed the epidemic rate $\left(r_{G}\right) 15-19 \%$, reduced the area under the disease progress curve (AUDPC) 29-49\%, and increased yield 13-17\%. Sprays with low rates also provided better control of disease (21\%) and higher yields (18\%) than applications of the full rate of fungicide according to a weather-based schedule (AU-Pnuts). Sanitation by crop rotation or fallow delayed epidemics of peanut leaf spots an additional 9 days and improved the suppression of the disease waves by the 
low fungicidal rates. In contrast, monoculture precluded fungicides to delay $t_{0}$ and to reduce $y_{0}$ for some pathosystems. Initiation of fungicidal treatments based on action thresholds $(0.0001$ to 0.05$)$ resulted in faster epidemic rates $(25 \%)$, higher AUDPCs (199\%), and lower yields (17\%) than those obtained with preventive sprays. Inclusion of early sprays of low rates in standard $(1 \times)$ calendar schedules improved the control of disease $48 \%$ and increased yield $18 \%$. The use of a partially resistant cultivar allowed the lengthening of spray intervals from 3.5 to 7 days $(0.25 \times$ rate $)$ and from 6 to 10 days $(0.5 \times$ rate) without affecting the control of disease significantly. Although the low fungicidal rates were applied on a more frequent basis than the standard $(1 \times)$ treatment, the total amount of fungicide used for each spray season was $8-25 \%$ less for the low rates than for the full rate. For some pathosystems, preventive applications of low rates of fungicide at short intervals may be a better approach to manage epidemics than calendar-, weather-, or threshold-based spray schedules. 


\section{CHAPTER I \\ INTRODUCTION}

Directness and practicality have made the reduction of the rate of disease progress by chemicals one of the most, if not the most, widely used method of disease control (Skylakakis, 1983). Because they were very effective, inexpensive, and readily available, fungicides became essential for the profitable production of many crops. With the increased costs and regulations associated with fungicide use, there is now a need for a more comprehensive understanding of how these compounds can be used more efficiently (De Waard et al., 1993). Additionally, the increasing concern about the environmental impact of fungicides has produced a need for more efficient approaches to maintain plant protection.

The sprays of protectant fungicides at long intervals of time fail to maintain an effective amount of fungicide on the leaf surface (Bruhn and Fry, 1982; Neely, 1970) and to protect the young plant parts that develop after each spray (Berger, 1973; Elliot and Spurr, 1993). Protectant fungicides are often applied late in the course of an epidemic, so that the chemical control has no effect on the established infections (Berger, 1973; Fry et al., 1979, van der Plank, 1963). Such situations seem very common for the traditional fungicidal spray programs, especially when timing of sprays is determined by action thresholds based on given amounts of disease. 
To improve chemical control of plant diseases, a new approach for management of epidemics with fungicides has been proposed (R. D. Berger, personal communication). This approach is based on the control of the initial waves of the epidemic by means of reduced rates of fungicides. The average speed of an epidemic is set by the intensity of the initial waves of disease, which, in turn, are determined by the basic infection rate $(R)$ (Berger, 1989; Oort, 1968). The basic infection rate is maximum in the early epidemic stages and then decreases over time (Berger, 1989; Campbell and Madden, 1990; Jeger, 1984; van der Plank, 1963). Control measures, such as fungicidal sprays, that reduce $R$ when it is normally maximum should result in delayed onset of disease, lower disease waves, and less severe epidemics.

Because of the small amount of inoculum available in the early stages of the disease, significant reduction of both the number $(N)$ and the efficiency $(e)$ of the fungal spores can be achieved with lower rates of fungicide. Reduced rates, applied repeatedly at short time intervals, can also protect the developing new tissues and decrease the epidemic rate over a long period. Additionally, if a broad-spectrum fungicide is used, the preventive application of reduced rates can prevent plant infection from multiple fungal species, which is not possible when action thresholds or forecast systems for specific diseases are used.

Control of initial waves of disease with reduced rates of fungicide has been recommended to manage epidemics of cercospora leaf blight on celery (Lacy et al., 1996). During the time when weather is very favorable for sporulation of, and infection by, the pathogen, the epidemics can be suppressed with frequent sprays of $0.25 \times$ rate of efficacious fungicides. Although this epidemiological principle of disease control should 
be effective for many other pathosystems, little information exists concerning the use of minimum spray intervals with low fungicidal rates to manage epidemics.

In this investigation, the potential of lower rates of fungicide to suppress and delay the initial waves of disease and to manage epidemics was tested in 18 experiments in the greenhouse or in the field. Black spot of roses, crown rust of oats, helminthosporium leaf spot of rye, peanut rust, and the early and late leaf spots of peanuts were used as disease models to test the lower-rate approach. The main objectives of this research were $i$, to test the potential of reduced rates of fungicide to control disease, $i i$, to determine how lower rates affect the epidemic waves and the disease-progress curve, iii, to compare lower-rate spray programs to calendar-, threshold-, and weather-based fungicidal schedules, $i v$, to evaluate the efficacy of mixed-rate spray programs to control disease, and $v$, to study the relationship of disease to yield for each of the above mentioned pathosystems. 


\section{CHAPTER II \\ LITERATURE REVIEW}

\section{Epidemic Progress}

An epidemic can be described as the progress of disease in time and space (Kranz, 1990). The progress in time may be expressed as $d_{y} / d_{t}=r y(1-y)$, where $d_{y} / d_{t}$ is the instantaneous logistic rate of disease increase at a specific time, $y$ is the proportion of diseased tissue, $r$ is the rate at which new disease appears, and (1-y) is the proportion of tissue without disease. For polycyclic pathogens, $y$ is variable, that is, new propagules produced on diseased tissues initiate new cycles of disease and increase the epidemic during the season (Campbell and Madden, 1990; Fry, 1982). At low levels of disease, the epidemic progress is exponential, since most plant tissues are healthy and available for infection. At higher levels, however, the epidemic progress is limited by the diminishing supply of non-infected tissue. Thus, the rate $\left(d_{y} / d_{t}\right)$ of disease increase declines, and the progress curve approaches some upper limit asymptotically.

The plotting of disease intensity versus time produces a disease progress curve which represents an integration of host, pathogen, and environmental effects that occurred during the epidemic (Campbell and Madden, 1990; Madden, 1986; Waggoner, 1986). Disease progress curves can be mathematically represented by means of nonlinear growth functions, or linearized according to transformation equations such as logit and gompit (Berger,1981a and 1981b; Madden, 1980; Waggoner, 1977). Several epidemiological parameters such as time of onset $\left(t_{0}\right)$; initial $\left(y_{0}\right)$, final $\left(y_{f}\right)$, and maximum $\left(y_{\max }\right)$ disease 
intensities; epidemic rate $(r)$; duration of the epidemic $(t)$; and area under the disease progress curve (AUDPC) are then determined and used for statistical analysis (Berger, 1988; Campbell and Madden, 1990; Reynolds and Neher, 1997). The interpretation of disease progress curves enables one to understand and predict the dynamic process of disease increase, compare epidemics, evaluate the effect of control measures on epidemics, and choose the best approaches for disease control (Berger, 1988; Madden and Campbell, 1990).

\section{Epidemic Waves}

The average speed of an epidemic seems to be set by the intensity of its initial waves (cycles of disease). For instance, high initial waves of disease on a few leaves or plants may indicate a subsequent, fast, and severe epidemic in the entire field (R. D. Berger, personal communication). Initial waves of disease have been observed either by simulation of epidemics (Zadoks, 1971) or by observation of natural epidemics of leaf diseases in alfalfa (Berger and Roberts, 1992).

Oort (1968) and Zadoks (1971), simulated the effects of the basic infection rate $(R)$, latent period $(p)$, and infectious period $(i)$ on the shape of the epidemic waves. When $p$ is long and $i$ is short, the successive waves are well established and do not overlap each other on a log scale. In the long run, however, the waves are damped and the disease increase approximates a straight line. If $p$ is short, the waves overlap each other at the outset and the increase results in a straight line from the beginning. In either case, the height of the waves is proportional to $R$. Combinations of high $R(\mathrm{~s})$ and short $p(\mathrm{~s})$ result in fast epidemics. 
Berger (1989) hypothesized that the shape of the epidemic waves depends on the shape of the curve describing the variable latency, in which individual infections flow through a series of substages, whose number and developmental rates determine the shape and duration of the curve. Thus, the higher the number of substages, the more flattened the waves. Additionally, Berger (1989) observed that the height of the waves is proportional to a true infection rate (analogous but not equal to $R$ ), the breadth of the waves is affected by the average latent period, and the rapidness of wave damping is dependent on the level of the initial disease and the length of each variable latency period.

\section{The Basic Infection Rate $(R)$}

The basic infection rate $(R)$ of van der Plank (1963) can be defined as the number of daughter lesions produced from one infectious parent lesion in one time unit (usually, one day) (Oort, 1968; Zadoks and Schein, 1979). The basic infection rate is a function of two factors: $i$, the infectiousness of the infectious tissue and $i i$, the susceptibility and vulnerability of the healthy tissue to infection (Van der Plank, 1967). These factors can be represented as $N$, the number of spores produced per unit area of sporulating leaf tissue, and $e$, the proportion of spores which germinate and start lesions after falling on healthy leaves (Zadoks and Schein, 1979). Both $N$ and $e$ are sensitive to environmental conditions, host resistance, and control measures (Berger, 1989; Van der Plank, 1967).

The basic infection rate decreases in the course of an epidemic as the proportion of infected tissue increases (Berger, 1989; van der Plank, 1963). According to Berger (1989), there are three main reasons for this behavior: $i$, the efficiency of each propagule is greatly reduced at late epidemic stages because many spores compete for few susceptible sites, $i i$, most susceptible sites are already infected, those sites that remain are 
more resistant to infection, and iii, in many pathosystems the sporulation is reduced per unit of diseased area because of competition for host nutrients. The modification of the microclimate within the plant canopy during the epidemic can be an additional reason for a decreasing $R$ (Bergamin Filho and Amorim, 1996; Campbell and Madden, 1990). In growing plants, the average value of $R$ remains high because of the new susceptible tissue that becomes available during the course of the epidemic.

\section{Strategies to Manage Epidemics}

Since the development of an epidemic depends on the amount of initial disease, the epidemic rate, and the time interval during which host and pathogen interact, Berger (1977) proposed three epidemiological strategies to minimize losses induced by plant diseases: $i$, eliminate or reduce initial inoculum $\left(y_{0}\right)$ or delay its appearance $\left(t_{0}\right), i i$, slow the rate of disease increase $(r)$, and $i i i$, shorten the time of exposure $(t)$ of the crop to the pathogen.

The elimination or reduction of the initial inoculum, termed sanitation, aims at decreasing the number of propagules of a pathogen before a crop is planted. Sanitation has been used primarily to manage monocyclic diseases, but examples of the effectiveness of sanitation for polycyclic diseases are also available (references given in Berger, 1977).

Slower rates of disease increase can be obtained by management or control practices directed to reduce the pathogen's inoculum during development of the epidemic, reduce inoculum transport and arrival, interrupt the pathogen life cycle, or manage the environment in such a way to make it less favorable to disease development (Berger, 1977). Chemicals and host resistance are the most used and effective means to slow the epidemic rate (Berger, 1977 and 1988; Fry, 1977 and 1982; Skylakakis, 1983; Van der Plank, 1967). 
Shortening of the time of exposure of the crop to the pathogen can be accomplished by managing the date of sowing or transplanting, using short-season cultivars, and maintaining adequate soil fertility and moisture. Superior control of the epidemic is obtained by combining control measures that reduce $y_{0}, r$, and $t$ simultaneously (Berger, 1977).

\section{Management of Epidemics with Fungicides}

Fungicides have commonly been applied to slow the rate of progress of an epidemic (Berger, 1977 and 1988). This occurs in direct proportion to their effect in reducing both the number $(N)$ and the efficiency $(e)$ of the fungal spores (Berger, 1977, 1988; Van der Plank, 1967). The effects exerted by fungicides on epidemics depend on how often they are used. If the chemical is applied only once or just a few times at the beginning of the epidemic, the major effect is to delay the onset of epidemic development. In this case, only the amount of initial disease is suppressed, but it may be sufficient to prevent economically important losses in some crops. If the treatment is repeated during the season, however, both the amount of initial disease and the rate of epidemic development are reduced. Since the efficacy of the secondary inoculum is affected, the pathogen population increases at a slower rate (Fry, 1977, 1982).

\section{Interpretation of Fungicidal Effects on Epidemics}

Zadoks (1971) described the theoretical interpretation of the effect of a protective fungicide on the epidemic of a leaf-spotting pathogen. According to his interpretation, the swing of disease into the effective period is a sharp process, followed by a zerodisease increase during the time of fungicidal activity, after which disease progress 
parallels that of non-treated plants. As pointed out by Berger (1977 and 1988), however, the actual disease response that follows the epidemic interruption does not usually resemble Zadoks' interpretation. Indeed, the disease has more of a gradual swing into the period of fungicidal effects and the rate of disease progress during the effective period may decrease on a growing crop. Also, the following upward swing of increasing disease is usually greater than that described by Zadoks, thus reducing the beneficial effect of the control treatment (Berger, 1975a).

The differences between the theoretical and the actual disease response to fungicides are influenced by factors such as plant growth, presence of latent infections, and the amount of host tissue available for infection (Berger, 1977 and 1988). The increase in healthy tissue associated with plant growth dilutes the proportion of diseased area. Latent infections are not affected by sprays of protective fungicides. Therefore, a minimum of one latent period is required to observe fungicidal effects on disease progress. Also, when the amount of healthy tissue is large, the number of sites available to the pathogen is great after the action of the fungicide has ceased. Thus, it is reasonable to expect higher rates of disease progress in these situations. These points have not been studied in detail for most pathosystems.

\section{Duration of Fungicidal Effects}

The period of crop protection that results from a single application of fungicide depends on how quickly the fungicidal deposit decays, how sensitive the fungal population is to the active ingredient, and the rate at which new unprotected foliage is produced (Elliot and Spurr, 1993). The fungicide may be removed by rain, wind, or mechanical abrasion, or it may be degraded by various chemical, physical, or biological 
agents (Neely, 1970). For instance, the residues of maneb did not prevent conidial germination of some phytopathogenic fungi one week after application (Neely, 1970). The half-retention of chlorothalonil on passion fruit leaves was as short as 2.6 days and 1.2 days under average daily rainfalls of $0.4 \mathrm{~cm}$ and $0.8 \mathrm{~cm}$, respectively (Ko et al., 1975). On potato leaves, the average half-life (6.6 days) of chlorothalonil was reduced to 1.23 days depending upon the environment (Bruhn and Fry, 1982). On peanut plants, the mean half-life of chlorothalonil averaged $3.8,4.8$, and 4.8 days in the top, middle, and bottom canopy layers, respectively (Brenneman et al., 1990). Also on peanut, Elliot and Spurr (1993) demonstrated the influence of new leaf emergence on the persistence of chlorothalonil on leaves. The residue half-life decreased from 13.6 days to 6.4 days on actively growing plants. Berger (1973) reported that maneb applied to sweet corn the day before spore deposition was ineffective to control Exserohilum turcicum, because infection took place on unprotected leaf tissue that emerged from the whorl in the meantime.

\section{Timing of Fungicidal Sprays}

In addition to the problem of residue decay, fungicides are frequently used too late in the course of the epidemic. They are often applied when symptoms of disease are first detected. At this time, there are many additional infections not yet visible, and these will be unaffected by protectant fungicides. For instance, Berger (1973) demonstrated that aerial sprays of maneb effectively controlled northern leaf blight (caused by E. turcicum) on sweet corn when applied on days of peak spore counts. Sprays applied $24 \mathrm{hr}$ after spore deposition were ineffective in controlling the same disease. In potato, maneb suppressed disease development of Phytophthora infestans when regular applications were begun before epidemics were established. If applications were made after the epidemic 
was in progress, retardation of the epidemic was detected only after 8 to 10 days (Fry et al., 1979). Thus, in a rapid epidemic, the first application of a protectant fungicide after symptoms become apparent is unlikely to be effective for commercial practice (van der Plank, 1963).

Protectant fungicides sprayed at long intervals of time, or even weekly intervals, may not be able to prevent infection and to slow disease development. Spores produced at the end of each latent period generate new infections and promote subsequent disease progress (van der Plank, 1963). For many diseases, especially those with rapid progress, the latent period is usually short, less than one week. Thus, many spores germinate and infect the plant before fungicides are reapplied. In such situations, fungicides should be sprayed at shorter time intervals, especially during periods of intense plant growth and weather favorable to disease development.

\section{Action thresholds to time fungicidal sprays}

Fungicides are often applied to plants based on the amount of disease observed. For example, an action threshold to manage sunflower rust was defined as the occurrence of $3 \%$ disease severity prior to the $27^{\text {th }}$ day after planting (Shtienberg, 1995). In Canada, fungicidal applications to control cercospora blight in carrot were recommended when the disease incidence reached 80 or $100 \%$ of plants with at least one lesion on the intermediate (middle) leaf for early varieties, and $50 \%$ for late varieties (Abraham et al., 1996). Campbell and Madden (1990) provide several other examples of disease control programs based on action thresholds.

Determination of action thresholds, however, may be problematic because of the various aspects it involves. For example, Mudita and Kushalappa (1993) reported that a 
disease threshold to initiate fungicidal applications to control septoria blight on celery could not be established because of the rapid disease progress and high yield loss associated with very low disease levels. Jong and Hasper (1996) proposed a tolerable leafinjury level to time fungicidal sprays to control powdery mildew on Brussels sprouts, but the mentioned injury level was not clearly determined. Additionally, disease thresholds do not take into account the numerous latent infections which increase the disease level before any control measure is taken. For instance, if the fungicidal sprays to control bean rust are delayed until the disease is about $1 \%$, enough latent disease is already present to advance the disease to nearly $50 \%$ within ten days (Berger, 1975b). More importantly, if the action threshold is higher than the amount of disease near the end of the first wave for a given epidemic, then the tempo of the epidemic has already been established (R. D. Berger, personal communication).

Weather-based fungicide schedules

Another important approach to time sprays relies on fungicidal applications when weather (temperature, rainfall, or duration of leaf wetness) is conducive to disease. Several advisory or forecast systems have been developed for this purpose. Examples are available for diseases on apple (Brown and Sutton, 1995), carrot (Abraham et al., 1996), celery (Lacy, 1994), grapes (Broome et al., 1995), lettuce (Scherm et al., 1995), peanut (Bailey et al., 1994; Brenneman and Culbreath, 1994; Davis et al., 1993; Jacobi et al., 1995; Linvill and Drye, 1995; Wu et al., 1996), potato (Johnson et al., 1996), tomato (Fulling et al., 1995; Gleason et al., 1995), and many other crops (examples given in Campbell and Madden, 1990). Some of these advisory systems combine weather information with the availability of pathogen propagules, the amount of initial disease, or 
the level of disease resistance (Fullin et al., 1995; Jacobi and Backman, 1995) to forecast disease.

The benefits of both threshold- and weather-based spray programs may be affected by the presence of multiple infections, occurrence of rapid weather changes, low efficiency of the available fungicide, and time required to implement the control measure. For example, Fulling et al. (1995) observed that weather-based fungicide schedules designed to control Colletotrichum coccodes in tomatoes do not account for other foliar diseases which can affect plants significantly. Similarly, the tolerable leaf-injury threshold for powdery mildew on Brussels sprouts (Jong and Hasper, 1996) should take into account the damage caused by other fungi such as Alternaria, Albugo, and Mycosphaerella. Abraham et al., (1996) pointed out that high-risk thresholds for control of cercospora blight of carrot should be recommended with caution because of the potential for a rapid increase in disease resulting from one, long, wet period or any delay in fungicide spray. Shtienberg (1995) observed that maneb, fenbuconazole, difenoconazole, and promoconazole were only partially effective to control sunflower rust once the $3 \%$ action threshold was reached. The same author mentioned that initiation of spraying at higher disease severities (e.g., 10\%) resulted in inadequate disease suppression. Wilks and Shen (1991) pointed out that most disease advisory systems are not predictive, so by the time disease-favorable weather conditions trigger a spray recommendation, the disease process is already under way. Also, the logistics of applying fungicides may further delay action for several more days. 


\section{Use of Reduced Rates of Fungicides to Control Disease}

The basic concept underlying chemical control of fungal pathogens is that the pathogen population must be kept at an economically acceptable low level but that it does not necessarily have to be eliminated (Zadoks, 1977). Adequate disease suppression can be achieved despite incomplete efficacy of a chemical application if the infection rate of a polycyclic-pathogen population is maintained at a very low level (Fry, 1982).

The efficiency of the chemical control of fungal pathogens is not linearly related to the fungicide dosage (Diamond et al., 1941; Fry, 1975, 1982; Miller, 1967), and an increase of the fungicidal rate over a certain level does not necessarily improve the control of the target disease. For instance, Labrinos and Nutter (1993) reported that $0.13 \times, 0.25 \times$, and $0.5 \times$ rates of chlorothalonil completely inhibited spore germination of Cercosporidium personatum in the laboratory, and there was no response to increased fungicidal concentrations when used to prevent plant infection. Bowen et ab (1994) observed no differences on the control efficiency of Entomosporium leaf spot on photinia by $0.25 \times, 0.5 \times$, and $1 \times$ rates of myclobutanil, tebuconazole, or diniconazole. Schein et al. (1984) reported that only $5 \mathrm{mg} \mathrm{l}^{-1}$ of triadimenol, whose suggested field dosage is 187.5 $\mathrm{mg} \mathrm{l}^{-1}$, reduced the colonies of Erysiphe graminis f. sp. tritici on wheat leaves to about $10 \%$ of the controls.

Reduced rates of fungicides do not control disease adequately unless sprayed at short intervals of time. For instance, Culbreath and Brenneman (1994) reported lower control of early leafspot and lower peanut yield when $0.25 \times$ and $0.5 \times$ chlorothalonil were applied to peanuts at 14-day intervals, which is actually the time interval for the full rate. Similar results were obtained by Aquino et al. (1992), who used lower rates of 
chlorothalonil at 14-day intervals to generate different intensities of late leafspot on peanuts.

Lower rates of fungicide can be combined with other strategies for disease control. For example, Fry (1975 and 1978) studied the integration of reduced rates of protectant fungicides with polygenic resistance of potato to Phytophthora infestans. In the less susceptible varieties, the disease was adequately controlled by an amount of fungicide about $40 \%$ lower. Sandler (1995) evaluated the efficacy of reduced rate combinations of chlorothalonil and an antitranspirant to control fruit rot in cranberries and concluded that several combinations provided control comparable to that with the fungicide used alone at suggested label rates. Lower fungicidal rates have also been used in mixtures of fungicides with different modes of action to delay or prevent control failures caused by fungicide resistance in pathogen populations (Sanders et al., 1985).

\section{Assessment of Fungicidal Effects}

The correct determination of the disease response to fungicides demands frequent, accurate, and precise disease assessments. These should start at the first sign of disease to provide information on the date of onset and features of the early epidemic stages, be made at time intervals no longer than one-fourth to one-third latent period for the initial disease cycles, and then continued at one-half to one latent-period intervals until no significant disease increase is observed (Berger, 1980 and 1988). Disease intensity should be expressed as percentage or proportion of host area diseased. A Horsfall-Barratt rating scale, modified by within class categories to obtain more accurate intraclass ratings (Berger, 1980), can be used to assess disease. For disease severities of less than $1 \%$, however, it is advisable to determine the actual number of lesions and their average size, 
and then divide the product of lesion number $\times$ lesion size by the total leaf area (Berger, 1980).

Data obtained from periodic disease assessments are used to generate the disease progress curve and to calculate the epidemic rate $(r)$. Berger (1988) suggested three ways to calculate $r: i$, the two-point method, where the first and last disease assessments are used to calculate the rate of disease progress either by the logistic $\left(r_{L}=\left[\operatorname{logit}\left(y_{\max }\right)-\operatorname{logit}\right.\right.$ $\left.\left.\left(y_{\min }\right)\right] /\left(t_{\max }-t_{\min }\right)\right)$ or Gompertz $\left(r_{G}=\left[\operatorname{gompit}\left(y_{\max }\right)-\operatorname{gompit}\left(y_{\min }\right)\right] /\left(t_{\max }-t_{\min }\right)\right)$ transformation equation, where logit $=\ln [y /(1-y)]$, gompit $=-\ln [-\ln (y)]$, and $t_{\max }-t_{\min }=$ differential time between the first and the last disease assessments, $i i$, the linear regression of the disease values transformed to either logit or gompit, and iii, the nonlinear fitting of growth functions to disease values.

Treatment comparisons based only on epidemic rates may be meaningless when the same rates change frequently during a short time, because of the epidemic interruption by fungicide spraying or unsuitable environment. For this reason, parameters such as the time of delays, the AUDPC, and the plotting of the derivative $d_{y} / d_{t}$ may be more appropriate to compare the effects of fungicide treatments (Berger, 1988). Fry (1978) found that AUDPC was more reliable than the apparent infection rate or final disease rating for the quantification of effects of fungicide on infection by $P$. infestans in potatoes. The AUDPC is an integration of disease intensities measured during the plant growth and its application seems to be adequate for diseases that reach high intensity in a short period of time (van der Plank, 1963). To account for epidemics of different duration, the AUDPC can be standardized by dividing the AUDPC value by the total time during which the disease was integrated (Campbell and Madden, 1990). 


\section{Fitting Models and Statistical Analysis of Disease Progress Curves}

The temporal analysis of epidemics relies on the characterization of the disease progress curves by mathematical models, whose parameters are then used for statistical analysis and comparison of epidemics (Campbell and Madden, 1990). Several growth functions have been used for this purpose (Berger, 1981a; Madden, 1980; Waggoner, 1977). Two of the most popular models are the logistic (van der Plank, 1963) and the Gompertz (Analytis, 1973; Berger, 1981a, 1981b), which have been used to describe epidemics of polycyclic diseases (van der Plank, 1963; Waggoner, 1986). Additional models less frequently used are the monomolecular (van der Plank, 1963), the Richards (Campbell and Madden, 1990), and the Weibull (Pennypacker et al., 1980). Polynomial regression has not been used to a great extent in epidemiology because the biological interpretation of the parameters is often not possible (Hau and Kranz, 1990; Madden, 1986)

\section{Growth Functions}

Application of the logistic model in epidemiology was proposed by van der Plank (1963). The logistic curve is defined by three epidemic components (Waggoner, 1986): $i$, the initial proportion of disease, $i i$, the rate at which the epidemic progresses, and $i i i$, the maximum attainable disease. The integrated logistic equation (Campbell and Madden, 1990) is $y=y_{\max } /\left[1+B \exp \left(-r_{L} t\right)\right]$, where $y$ is disease proportion, $y_{\max }$ is the asymptote or theoretical maximum value for $y, r_{L}$ is the epidemic rate, $t$ is time, and $B$ is a constant of integration written as $\left(1-y_{0}\right) / y_{0}$, where $y_{0}$ is the disease proportion at $t_{0}$ (first disease assessment). A logistic curve is sigmoid and symmetric at its inflection point $(y=0.5)$ 
(Waggoner, 1986). The logistic equation can be linearized to logit $(y)=\operatorname{logit}\left(y_{0}\right)+r_{L} t$, where logit $=\ln [y /(1-y)]($ Campbell and Madden, 1990).

The Gompertz model was first used in epidemiology by Analytis (1973). Later, Berger (1981a) reiterated the appropriateness of this model to describe disease progress curves. The Gompertz model is defined by the same epidemic components previously mentioned for logistic. The integrated Gompertz equation is written as $y=y_{\max }$ \{exp [-B $\left.\left.\exp \left(-r_{G} t\right)\right]\right\}$, where $y, y_{\max }, r_{G}$, and $t$ are as described above, and $B=-\ln \left(y_{0}\right)$. This model is also sigmoid but not symmetric at its inflection point $(y=0.37)$. The plot of the derivative is skewed to the right. The Gompertz linearized equation is as gompit $(y)=$ gompit $\left(y_{0}\right)+r_{G} t$, where gompit $=-\ln [-\ln (y)]($ Berger, 1981a, 1981b).

\section{Estimation of Model Parameters}

Parameters for both linear and nonlinear models can be estimated by means of least-squares regression analysis (Berger, 1988; Campbell and Madden, 1990; Draper and Smith, 1981; Montgomery, 1991; Neter et al., 1985). In this procedure, the values for the estimates are chosen in such way to minimize the sum of squares for error (SSE), that is, the squares of the differences between the observed and the predicted response variable (y). The number of observations for $y$ must be at least equal to the number of parameters in the model (Montgomery, 1991). Although the logistic and Gompertz models have only three parameters, many values $(>10)$ are required to obtain more realistic estimates of epidemic rates (Berger, 1988). Standard software packages such as PlotIT (Scientific Programming Enterprises, Haslett, MI) and SAS (Statistical Analysis Systems, SAS Institute Inc., Cary NC) provide the means to estimate parameters for both linear and nonlinear models. 


\section{Model Evaluation}

A valuable measure of the goodness-of-fit of a regression model is given by the coefficient of determination $\left(R^{2}\right)$, which represents the proportion of the total variation on the response variable $y$ associated with the use of the regressor variable $x$ (Montgomery, 1991; Neter et al., 1985). For both linear and nonlinear models, $R^{2}$ can be calculated as $R^{2}=1-(\mathrm{SSE} / \mathrm{SST})$, where SSE $=$ sum of squares for the error term and SST $=$ sum of squares for totals (Cornell and Berger, 1987; Madden and Campbell, 1990; Neter et al., 1985).

Another important approach to evaluate models is to analyze the distribution of the residuals for the response variable $y$ plotted versus $x$ or predicted $y$. If the residuals are randomly distributed, then the model adequately fits the relationship between $y$ and $x$. In contrast, the occurrence of systematic patterns such as U- or S-shape scatter of points indicates that the model does not fit the data properly (Campbell and Madden, 1990; Hau and Kranz, 1990; Madden, 1986; Montgomery, 1991). Additionally, a low sum of squares for the residuals indicates close prediction of the response variable $y$ by the model being used.

The goodness-of-fit of a regression model should also be tested by means of a lack-of-fit test. In this case, the sum of squares for the error term (SSE) is partitioned into the sum of squares attributable to pure experimental error (SSPE) and the sum of squares due to lack of fit (SSLOF) of the model. If the $F$-test for lack-of-fit is not significant, then there is no apparent reason to doubt the adequacy of the regression model (Montgomery, 1991). To run a lack-of-fit test, repeated observations of $y$ at each $x$ are required. Finally, it should be pointed out that regression models should never be used 
for extrapolation, since the regression relationships are valid only for values of the regressor variable $(x)$ within the range of the original data (Montgomery, 1991).

\section{Comparison of Model Parameters}

Epidemic parameters or model estimates can be examined for statistical differences between treatments or naturally occurring epidemics. Confidence intervals for the difference between two estimates can be approximated based on the standard error $(s)$ of the difference and on a critical $t$ value obtained from reference $t$-tables (Montgomery, 1991; Neter et al., 1985). If the confidence interval does not include zero, then the hypothesis of equality can be rejected. This approach can be expanded to compare three or more values in a pairwise fashion. This procedure, however, increases the overall significance level of the test, thus reducing its overall precision (Madden, 1986).

Analysis of variance (ANOVA) is an alternative approach to analyze data from experiments divided into blocks or replications. In this case, the levels of $y$ for all replicates at each time are used in a regression analysis and the mean values for each estimate are compared using a pooled estimate of the underlying variance. Alternatively, a regression analysis can be performed for each replication separately, and an ANOVA is calculated, from which a test of the significance of the estimated parameters can be made (Madden, 1986).

\section{Comparison of Treatment Means}

If the analysis of variance for any variable being tested is significant, then there are significant differences between the means of the variables (Montgomery, 1991). For 
a quantitative variable tested at various levels, regression analyses or curve fitting should be performed to determine how the variable changes as a function of the treatments (Johnson and Berger, 1982). Otherwise, contrasts or multiple comparison tests can be applied to identify the differing means. Contrast procedures, e.g., orthogonal contrasts, are usually pre-planned tests chosen prior to running the experiment and examining the data. Multiple-comparison procedures are post-comparison tests such as Scheffe's significant difference, Fisher's least significant difference, or Duncan's multiple range test (Gilligan, 1986; Hau and Kranz, 1990; Johnson and Berger, 1982; Madden et al., 1982; Montgomery, 1991). Among the post-comparison tests, Fisher's has higher correct-decision rates and should be preferred whenever multiple-comparison procedures are applicable (Madden et al., 1982).

When dependent disease data collected over time at discrete, uniform intervals can be arranged in temporal order, a different statistical procedure called time-series analysis can be used (Scherm and Yang, 1997). Time-series analysis is useful to identify patterns (trends, stationary, autocorrelation, or cyclic behavior) in time-series data. It also can be used for disease prediction, as an alternative or complement to procedures based on regression or simulation (Yang and Zeng, 1992). 


\section{CHAPTER III \\ MANAGEMENT OF EPIDEMICS OF CROWN RUST ON OATS}

\section{Introduction}

Crown rust, caused by Puccinia coronata f.sp. avenae, is one of the most important diseases of oats (Avena sativa L.) worldwide (Clifford, 1995; Simons, 1970 and 1986). This disease significantly decreases the amount of grain produced by a plant. For instance, each percent increase in crown rust from 0 to $30 \%$ can reduce the kernel yield of susceptible varieties $40-50 \mathrm{~kg} \mathrm{ha}^{-1}$ (Bissonnette et al., 1994, Forcelini et al., 1993). Like other cereal rusts, crown rust may also affect the quality of the kemels, thus reducing their market value (Roelfs, 1986).

In the United States, oat crown rust is controlled by genetic resistance and only in a few cases are fungicides needed (Shaner, 1991). In Florida, a foliar spray program to control oat crown rust is usually not advised (Kucharek, 1988; Kucharek, 1994a). In some oat-producing areas of the world, however, the chemical control of crown rust is essential to obtain high yields from susceptible, high-quality-kernel varieties (Martinelli et al., 1984; Picinini and Fernandes, 1989). For instance, in Brazil, two sprays of a systemic fungicide increased the average kernel yield of 17 oat varieties from 2061 to $3293 \mathrm{~kg} \mathrm{ha}^{-1}$ (Severo et al., 1992 and 1993). This gain in yield can be even higher if the chemical control is improved by application of alternative epidemiological approaches.

In addition to the importance of crown rust itself, studies of this pathosystem provide insights on the management of rust epidemics on other cereals. Puccinia 
coronata $\mathrm{f}$. sp. avenae requires little moisture (as low as 5 hours of dew at $21^{\circ} \mathrm{C}$ ) to germinate and infect the plants (Politowski and Browning, 1975). This fungus has a latent period as short as 5 days at $25^{\circ} \mathrm{C}$ (Kucharek, 1988; Tollenaar, 1985), and it sporulates profusely for a long period. Van der Plank (1967) pointed out that the long $i$ (infectious period), the great $R$, and the resulting great $i R$ of the cereal rusts make their control very difficult on susceptible varieties and during weather favorable to disease. Therefore, the crown rust-oat pathosystem represents a useful model to study new approaches to manage epidemics.

The main objective of this research was to evaluate spray programs based on application of low fungicidal rates to control the initial waves of disease and to manage oat crown rust. Additionally, preventive applications of low fungicidal rates were compared to threshold-based schedules, periods of fungicidal treatment required to achieve satisfactory control of crown rust were assessed, and alternative spray programs were proposed.

\section{Material and Methods}

A total of seven experiments were conducted on the oat-crown rust pathosystem to test the viability of low fungicidal rates to control initial disease waves and to manage crown rust epidemics. Two of these experiments were conducted in greenhouse conditions in 1994-95 and the five others were developed in the field during the cropping seasons of 1995, 1996, and 1997.

\section{Experiments in the Greenhouse}

The experiments in the greenhouse were designed to evaluate the potential of low rates of fungicide to protect oat plants from increased inoculum densities of $P$. c. avenae 
and to assess the control of crown rust provided by applications of a full and a reduced rate of fungicide under daily, controlled inoculations of the crown rust fungus.

\section{Fungicidal rate vs. inoculum density}

Oat seeds were planted in plastic pots $(12 \mathrm{~cm}$ diameter $\times 15 \mathrm{~cm}$ high $)$ containing growing medium (Metro-Mix 300 - Scotts-Sierra Horticultural Products Co., Marysville, $\mathrm{OH})$ on 22 December 1994. After germination, the seedlings were thinned to allow 30 plants equally spaced in each pot. For each combination of fungicide concentration and inoculum density, a set of four pots was prepared, each pot being a replication. The pots were maintained in the greenhouse and watered as needed. Twice a week, the irrigation water was supplemented with a $15-30-15$ (N-P-K) fertilizer at the ratio of $0.1 \%$. Additional plants were grown under the same conditions and used for leaf area measurements by means of a leaf area meter (model LI-3000, LI-COR Inc., Lincoln, NE 68504).

About 10 days after seeding, the unfolded leaves were marked with a permanent color pen at the base of the unfolded area for future disease assessment. The plants were then sprayed with $0 \times, 0.125 \times, 0.25 \times, 0.5 \times, 0.75 \times$, and $1 \times$ the label rate of mancozeb (Manzate 200DF, Du Pont Agricultural Products, Wilmington, DE) and left to dry for at least 2 hours. In the meantime, suspensions of fresh uredospores were prepared by successive dilution in sterile water to achieve inoculum densities of ca. $5 \times 10^{3}, 1 \times 10^{4}, 2 \times$ $10^{4}$, and $4 \times 10^{4}$ uredospores $\mathrm{ml}^{-1}$. A 100 -ml-sample of each suspension was sprayed onto plants previously treated or not with fungicide. Immediately after inoculation, two leaves per pot were carefully cut, slightly dried, and used to estimate the number of spores applied to each square centimeter of the leaf surface. This was done by counting the 
uredospores attached to the adaxial surface of each leaf in a $0.5 \mathrm{~cm} \times 2 \mathrm{~cm}$ microscopic field at the mid-point of the leaf lengthwise. Inoculated plants were covered with plastic bags for 18 hours ( 12 hours in the dark and 6 hours in sunlight) and incubated at $21-25^{\circ} \mathrm{C}$ (Luke et al., 1984) in the greenhouse. The pots were arranged according to a completely randomized design. Ten days after inoculation, the number of pustules per marked leaf was quantified using a measuring magnifier (Bausch \& Lomb, Inc., Rochester, NY) with $0.1 \mathrm{~mm}$-scale intervals. The average leaf area was also determined by passing the leaves through a leaf area meter. The disease efficiency (\%) for $P$. c. avenae was then calculated based on the ratio of number of lesions per $\mathrm{cm}^{2}$ of leaf $\times 100$ to the number of uredospores inoculated per $\mathrm{cm}^{2}$ of leaf.

\section{Full vs. low rates of fungicide}

Oat seeds were sown in pots on 9 January 1995 and grown as described above. Three rates of the fungicide mancozeb $(0 \times, 0.25 \times$, and $1 \times)$ were sprayed onto plants for the first time at 10 days after planting. The $0.25 \times$ rate was then reapplied on days 12,14 , $17,19,21$, and 24 , whereas the $1 \times$ rate was repeated on days 17 and 24 only. Plants of the $0 \times$ rate treatment were sprayed with water on days $12,14,17,19,21$, and 24 . All fungicidal sprays were carried out at least 2 hours before inoculation.

A suspension of 20,000 spores per $\mathrm{ml}$ was prepared in sterile water plus two drops of Tween-20 for each $100 \mathrm{ml}$ of suspension. A volume of $360 \mathrm{ml}$, sufficient for 3 days of inoculation, was standardized and stored in the refrigerator. Each day, a 40-ml inoculum sample was taken and applied to 120 plants (four pots with 30 plants each) of each treatment. Plants in each pot were inoculated only once. New spore suspensions were prepared every 4 days. The inoculations were done in late afternoon and the plants were 
incubated as described above. The plants not inoculated were misted with water only. The subsequent procedures were similar to those described in the inoculum density experiment.

Since the viability of the inoculum could be affected by storing the spore suspensions in the refrigerator, this experiment was also conducted according to a different protocol. In this latter protocol, oat plants were sown on 9 January 1995 and sprayed with fungicides or water (controls) on $10,11,12,13,14,15,16$, or 17 days after planting. Several combinations of time intervals between fungicidal spray and inoculation were then achieved by day 17 , when all plants were inoculated. The fungicide was reapplied to plants in the $0.25 \times$ treatment every $2-3$ days to fulfill the protocol. Each treatment was a set of four pots with 30 plants each. Both incubation and evaluation followed the steps described previously for the other tests.

\section{Experiments in the Field}

Experiments on oats were conducted at the Green Acres Experiment Farm of the University of Florida, in Alachua County, FL, in 1995, and at a field area located southwest of Lake Alice, in Gainesville, FL, in 1996 and 1997. The cultivar Fulghum, susceptible to crown rust (Berger and Luke, 1979; Luke and Berger, 1982), was planted in all 3 years. In 1997, two additional experiments were conducted with Red Rustproof, a slow-rusting variety of oats (Berger and Luke, 1979), but not assessed because of low levels of disease. The top soil layer was plowed to a $0.20 \mathrm{~m}$ depth, $450 \mathrm{~kg} \mathrm{ha}^{-1}$ of a 10 10-10 (N-P-K) fertilizer was spread, and the soil was disked again to a $0.15 \mathrm{~m}$ depth. A supplement of ammonium nitrate $\left(118 \mathrm{~kg} \mathrm{ha}^{-1}\right)$ was spread on soil surface about 40 days after sowing. Plots $(1 \times 1 \mathrm{~m})$ were composed of five rows $(0.20 \mathrm{~m}$ apart $)$ and separated 
by an uncultivated border of $1 \mathrm{~m}$. For the 1995 experiment, a 2 m-wide border of rye (Secale cereale L.) around each experiment was established 2 weeks before planting. Plots were arranged in a complete randomized block design with four (1995) or five replicates (1996 and 1997). Oats were planted manually in 3-5 cm deep furrows with enough seeds to provide 300 plants per plot. Excess plants were thinned 30 days after planting. Weeds were removed manually every week whereas insects were controlled chemically as needed. Oat plants in these plots were inoculated at growth stage 26 (Zadoks et al., 1974) (3 in the Feekes scale; Large, 1954) either by a pot with 30 diseased plants (about 1000 pustules per pot) placed at the center of each plot (1995) or by direct spray of spore suspensions containing at least $4 \times 10^{4}$ uredospores $\mathrm{ml}^{-1}$ (1996 and 1997). The fungicide was delivered by means of a backpack sprayer (Spraying Systems Co., Wheaton, $\mathrm{IL}$ ), propelled by $\mathrm{CO}_{2}$, and connected to a 2-meter spray boom with six Conejet (hollow cone spray tip) nozzles. The spray pressure $\left(2.8\right.$ to $\left.3.5 \mathrm{~kg} \mathrm{~cm}^{-2}\right)$ and the operator's speed were adjusted to deliver a volume of $1501 \mathrm{ha}^{-1}$ (Dr. Tom Kucharek, personal communication). The fungicide mancozeb was used in all experiments on oats. The fungicidal rates ranged from $0 \times$ (no fungicide) to $0.25 \times\left(0.45 \mathrm{~kg}\right.$ a.i. ha $\left.{ }^{-1}\right)$, to $0.5 \times$ $\left(0.9 \mathrm{~kg}\right.$ a.i. ha $\left.{ }^{-1}\right)$, or to $1 \times\left(1.8 \mathrm{~kg}\right.$ a.i. ha $\left.{ }^{-1}\right)$ depending upon the purpose of each test. Disease severity was assessed twice a week or even daily as described for each experiment. In the early stages of the epidemics the diseased leaf area was estimated based on the number and size of lesions. The average pustule diameter measured in ten pustules at each disease assessment date was used to calculate the pustule area by means of the formula $\pi\left(d^{2} / 4\right)$, where $d=$ pustule diameter (mm) (Kochman and Brown, 1975). At the same time, ten plants were collected at random and their leaves passed through a 
leaf area meter to determine the total leaf area per plant. The diseased area was then divided by the total leaf area to determine the proportion of disease severity. In the later stages of disease development, the disease severity was assessed by visual evaluation of four leaves (flag, $\mathrm{f}-1, \mathrm{f}-2$, and $\mathrm{f}-3$ ) in each plant, and five plants per plot. Disease levels were rated according to the Horsfall-Barratt scale with the modifications suggested by Berger (1980). Plant growth was evaluated weekly based on plant height (cm) to the top leaf as well as the decimal code scale for plant growth (Zadoks et al., 1974). At maturity, plants were harvested, the grains cleaned, and the yield determined $\left(\mathrm{kg} \mathrm{ha}^{-1}\right)$.

Preventive spray vs. action thresholds

Although the effect of a fungicide on disease control is greatly affected by the amount of disease at the time of the first spray (Berger, 1973; Fry et al., 1979; van der Plank, 1963), fungicides are frequently applied to plants according to action thresholds based on the amount of disease observed (Abraham, 1995; Jong, 1996; Shtienberg, 1995). The detection of such thresholds is often problematic, since epidemics progress fast in their early stages and the amount of latent infections is unknown (Berger, 1975a and 1975b). Additionally, protectant fungicides do not control the pre-established infections. For such reasons, the use of action thresholds for decision making in disease management is sometimes risky.

Two experiments were conducted to evaluate the control of oat crown rust under preventive applications of low fungicidal rates and threshold-based schedules. Fungicidal spray programs, using a full $(1 \times)$ or a reduced $(0.25 \times)$ rate of mancozeb, were initiated at various levels of disease severity. These were compared to a zero-disease threshold, which represented the preventive application of either rate. The first experiment was 
established on 19 November 1994 and inoculated on 10 February 1995. Fungicidal treatments were initiated at the 0 (at inoculation), $0.0001,0.001,0.01$, and 0.05 disease severities and repeated once $(1 \times$ rate $)$ or three times $(0.25 \times$ rate) a week. The various spray programs tested in this experiment were discontinued 3 weeks prior to harvest ( 20 April 1995). The second experiment was planted on 4 December 1996 and inoculated on 15 February 1997. This trial did not include the 0.05 action threshold. Also, the frequency of application of the lower fungicidal rate was reduced from three to two sprays a week. Spray programs were discontinued at 35 to 38 days after inoculation. The oat plants were harvested on 23 April 1997.

\section{Period of fungicidal protection required for effective control of crown rust}

The period during which fungicidal protection is required to prevent disease depends on the host susceptibility, pathogen virulence, and weather conditions (Elliot and Spurr, 1993). This period may be short or long, but it rarely is over the entire plant cycle. After fungicide sprays have ceased, however, the amount of healthy and unprotected tissue is large, and this tissue presents numerous sites available for infection. In some situations, the rapid disease increase that takes place on the formerly protected tissue may reduce the beneficial effect of the control treatment (Berger, 1975, 1977, and 1988; Keinath, 1995, van Eeckhout et al., 1991).

An experiment was designed to determine the period of fungicidal treatment required to achieve satisfactory control of the disease. The experimental plots were planted on 3 December 1994 and inoculated on 10 February 1995. All fungicide treatments were initiated on the day of inoculation, repeated weekly (1× rate) or three 
times a week $(0.25 \times$ rate) for $3,4,5,6$, or 7 weeks, and then discontinued. The oat plants were harvested on 20 April 1995.

\section{Mixed spray programs}

The information obtained in the previous tests in greenhouse and in the field was used to design experiments on mixed spray programs. The usual fungicidal spray schedule, based on applications of the $1 \times$ rate at 10- to 12-day intervals from growth stage 32 ( 7 in the Feekes scale), was modified by replacing the first spray with preventive applications of the $0.25 \times$ rate twice a week. The objective of this combined program was to improve the control of the initial waves of disease and to optimize the management of the epidemics.

Experiments on mixed spray programs were conducted in the 1996 and 1997 cropping seasons. In the 1996 experiment, the planting and inoculation dates were 2 December 1995 and 20 February 1996, respectively. Because of the low temperatures that followed the first inoculation, a second inoculation was done on 13 March 1996. Several fungicidal spray programs were then initiated and evaluated for the control of oat crown rust. The Florida disease control program for wheat (Kucharek and Luke, 1989, Kucharek, 1994c) was adapted to oat and used as the standard program for chemical control of crown rust. The standard program is based on three applications of mancozeb (Manzate $200 \mathrm{DF})$, at $1.8 \mathrm{~kg}$ a.i. $\mathrm{ha}^{-1}$, one at the plant growth stage 32 (7 in the Feekes scale) and two others at 10- to 14-day intervals. In the 1996 experiment, the first fungicidal spray was omitted since no disease was detected at the plant growth stage 32 and the mean air temperature was still low. Thus, the standard treatment was restricted to two sprays of fungicide carried out at 109 (20 March 1996) and 120 days (31 March 
1996) after planting. The standard spray program was then compared to a $0.5 \times$ rate treatment, which was initiated 100 days (11 March 1996) after planting and repeated four times at weekly intervals, and to several fungicidal schedules based on applications of the $0.25 \times$ rate singly or combined with other rates as follows: $i$, a treatment based on seven sprays of the $0.25 \times$ rate between 100 days (11 March 1996) (second inoculation) and 126 days (6 April 1996) after planting, $i i$, a delay treatment (0.25×-delay) applied three times between 116 (27 March 1996) and 126 days (6 April 1996) after planting, iii, two shortterm treatments applied four $(0.25 \times, 4)$ or six times $(0.25 \times, 6)$ at semiweekly intervals after inoculation, and $i v$, three mixed treatments, called mixed I (four sprays of $0.25 \times$ plus two sprays of $0.5 \times$ ), mixed II (four sprays of $0.25 \times$ plus one spray of $1 \times$ ), and mixed III (six sprays of $0.25 \times$ plus one spray of $0.5 \times$ ), initiated 100 days (11 March 1996) after planting. This experiment was harvested on 22 April 1996.

The 1997 experiment was planted on 4 December 1996 and inoculated on 15 February 1997. The five treatments evaluated in this test were $i$, a nonsprayed control, $i i$, a standard schedule with three sprays of the $1 \times$ rate initiated at the growth stage 32 ( 7 in the Feekes scale), $i i i$, a single-rate treatment, which corresponded to 12 sprays of the $0.25 \times$ rate, $i v$, a mixed rate program (mixed I), which included four sprays of the $0.25 \times$ rate plus two sprays of the $1 \times$ rate, and $\nu$, another mixed rate program based on six sprays of $0.25 \times$ mancozeb plus two sprays of the full rate. All spray programs that included the lower fungicidal rate were initiated at inoculation. The frequency of application was twice a week for the $0.25 \times$ rate and $10-12$ days for the $1 \times$ rate. The oat plants were harvested on 23 April 1997. 


\section{$\underline{\text { Relationship between AUDPC and yield }}$}

Because of the various combinations of fungicidal rates, time intervals between sprays, and action thresholds tested in the experiments on the oat-crown rust pathosystem, several epidemics were generated for the same disease. Yields of oats from these epidemics were obtained and used to study the relationship between disease and crop loss. The procedures used in this study are described under statistical analysis of crop yield.

\section{$\underline{\text { Statistical Analyses }}$}

\section{Epidemic variables}

In each experiment, the treatments were analyzed in terms of time of onset $\left(t_{0}\right)$ of the epidemics, proportion of initial $\left(y_{0}\right)$ and final disease severities $\left(y_{f}\right)$, calculated $\left(r_{G}\right)$ or estimated epidemic rates $\left(r_{G N L} \mathrm{~s}\right)$, and areas under the disease-progress curves (AUDPC). The $t_{0}$ was the day after planting or after the inoculation on which the rust pustules were first detected. The amount of disease on $t_{0}$ was regarded as $y_{0}$. The $y_{f}$ was the amount of disease at the end of the season or at the last disease assessment. The epidemic rate $\left(r_{G N L}\right)$ was estimated by nonlinear regression of disease proportions against time using the Gompertz model. In the 1995 experiments, however, nonlinear regression analyses did not provide good estimate of the rate parameter, which was then calculated according to the two-point method described by Berger (1988). Epidemic rates calculated by the latter method are denoted by $r_{G}$. The AUDPC was calculated using the formula $\Sigma\left[\left(y_{i}+y_{i+1}\right) /\right.$ 2] $\times d_{t i}$, where $d_{t i}$ is the time interval between the observations $y_{i}$ and $y_{i+1}$ (Berger, 1988). The individual replicate AUDPC values were subjected to analysis of variance and regression analysis or multiple comparison procedures. The effects of action thresholds 
and duration of fungicidal programs on $r_{G N L}, y_{\beta}$ AUDPC, and yield were analyzed using multiple linear regression as implemented in Plot IT (Scientific Programming Enterprises, 1993). The general linear model $\mathrm{Y}=\mathrm{A} \mathrm{B}$, where $\mathrm{A}=$ treatment effect and $\mathrm{B}=$ block effect, was used to estimate and test the effects of spray programs on epidemic parameters. Computations were done in SAS-PC (SAS Institute, Inc., 1985) using PROC GLM. The treatment means were compared by the Duncan's Multiple Range Test at the $P=0.05$ type I error rate (Montgomery, 1991).

\section{Disease progress curves}

The disease progress curves were analyzed using the logistic or Gompertz nonlinear models, whichever model provided better curve fitting. The logistic model is $y_{t}$ $=y_{\max } /\left[1+B \exp \left(-r_{L} t\right)\right]$, whereas the Gompertz model is $y_{t}=y_{\max }\left\{\exp \left[-B \exp \left(-r_{G} t\right)\right]\right\}$. In each model, $y_{t}$ is disease proportion, $t$ is time, and $y_{\max }$ is the asymptote or theoretical maximum value for $y$. The constant of integration, $\left(1-y_{0}\right) / y_{0}$ for logistic or $-\ln \left(y_{0}\right)$ for Gompertz, is denoted by the parameter $B$, whereas $r$ denotes the epidemic rate $\left(r_{L}\right.$ for logistic or $r_{G}$ for Gompertz). The parameters $y_{\max }, B$, and $r$ were estimated using the procedure PROC NLIN METHOD=DUD in SAS-PC. The goodness of fit for the nonlinear models was evaluated based on the coefficient of determination $\left(R^{2}\right)$, calculated as $R^{2}=1$ - (SSE / SST), where SST is sum of squares total (Cornell and Berger, 1987), as well as the distribution of residuals and the sum of squares for error (SSE) (Campbell and Madden, 1990; Hau and Kranz, 1990).

\section{Relationship of disease to crop yield}

The relationship of disease, expressed as AUDPC, to oat yield was analyzed by multiple linear regression in Plot IT. The AUDPC was the independent variable $\mathrm{X}$ and 
crop yield was the dependent variable $\mathrm{Y}$. Oat yields were also submitted to analysis of variance for statistical effects of the qualitative spray programs. This was run in SAS-PC using PROC GLM, model $\mathrm{Y}=\mathrm{A} \mathrm{B}$, where $\mathrm{A}=$ treatment effect and $\mathrm{B}=$ block effect. Upon significance of the ANOVA $F$ test, the treatment means were further separated using the Duncan's Multiple Range Test at the $P=0.05$ type I error rate.

\section{Disease efficiency}

The disease efficiency of $P . c$. avenae on oats was regressed against inoculum density. Computations were done in Plot IT.

\section{$\underline{\text { Results }}$}

\section{Disease Efficiency of Puccinia coronata}

The disease efficiency $(e)$ of $P$. coronata to oats increased proportionally to the number of uredospores $(N)$ inoculated onto plants (Fig. 3.1). On unsprayed plants, $e$ ranged from 0.41 to $4.42 \%$ for $N$ between $5.5 \times 10^{3}$ and $4.4 \times 10^{4}$ spores $\mathrm{ml}^{-1}$. The relationship of $e$ to $N$ was represented by the quadratic equation $Y=73.47-38.88 X+$ $5.171 X^{2}, R^{2}=0.99$, in which $Y$ is percent efficiency $(e)$ and $X$ is the natural logarithm of $N$.

Applications of fungicides prior to inoculation reduced $e$ substantially (Fig. 3.1). Fungicidal treatments were more effective as high rates were used to control low inoculum densities. Fungicidal rates as low as $0.125 \times$ and $0.25 \times$ completely prevented infection of oats by the crown rust fungus at inoculum densities up to $1.1 \times 10^{4}$ uredospores $\mathrm{ml}^{-1}$. 


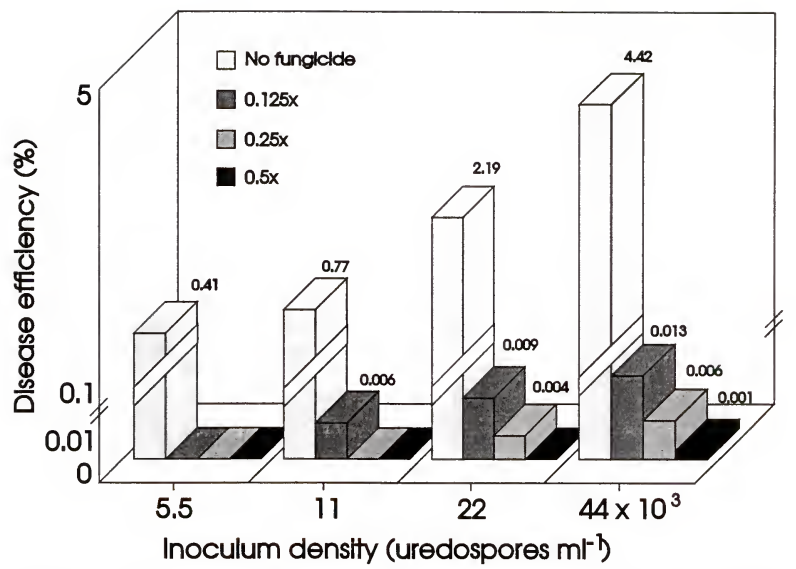

Figure 3.1. Disease efficiency of Puccinia coronata f.sp. avenae on oats under various inoculum densities and rates of fungicide. The $0.125 \times, 0.25 \times$, and $0.5 \times$ rates of mancozeb were applied to oat plants two hours prior to inoculation. Experiment developed in the greenhouse in 1995. 
The disease efficiency of $P$. coronata on plants subjected to single applications of fungicide ( $1 \times$ rate) increased as the fungicidal deposit on the leaf surface decayed (Fig. 3.2). For instance, $e$ increased from zero to $0.06 \%$ after the first spray, and from zero to $0.04 \%$ following the second spray of fungicide. Any increase in disease efficiency of $P$. coronata was prevented by applications of the lower rate of fungicide $(0.25 \times)$ at shorter spray intervals, which kept $e$ between zero and $0.01 \%$ all of the time.

In the experiment in which plants were sprayed with $1 \times$ or $0.25 \times$ rates at various days prior to a single inoculation of the crown rust fungus, the disease efficiency of $P$. coronata on unsprayed plants was $4.05 \%$. On plants sprayed with the $1 \times$ rate once a week, $e$ increased from zero to $0.1 \%$ as the time interval between fungicidal spray and inoculation increased from one to six days (Fig.3.3). Sprays of the $0.25 \times$ rate three times a week resulted in disease efficiencies in the range of zero to $0.01 \%$.

\section{Progress of Oat Crown Rust in the Field}

In the 1994/95 growing season, two experiments (1995-A and 1995-B) on the oatcrown rust pathosystem were conducted side-by-side in the field. In both experiments, the first pustules of rust were detected 9 days after inoculation. Therefore, the latent period $(p)$ for crown rust was referred to as $p=9$.

The daily increments of disease severity $\left(d_{y} / d_{t}\right)$, estimated by dividing the difference of disease proportion by the time interval between two disease assessments, were high at the end of each latent period (Fig. 3.4). Peaks of disease increase were observed around $18,29,36,44$, and 52 days after inoculation. The average time interval between peaks was about 8 days. In both experiments, the maximum increment in crown 


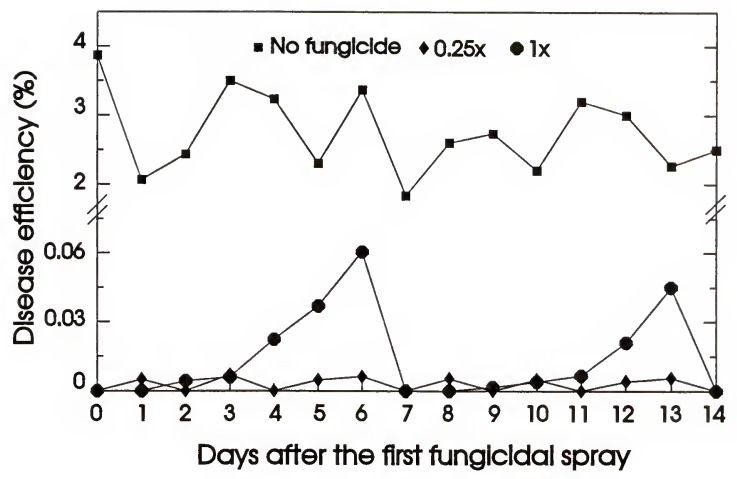

Figure 3.2. Disease efficiency of Puccinia coronata f.sp. avenae on oats plants subjected to two programs of fungicidal sprays in the greenhouse in 1995 . The $0.25 \times$ rate of mancozeb was applied on days $0,2,4,7,9,11$, and 14 . The standard $(1 \times)$ fungicidal rate was sprayed on days 0,7 , and 14 . Plants were inoculated daily with $2 \times 10^{4}$ uredospores $\mathrm{ml}^{-1}$. 


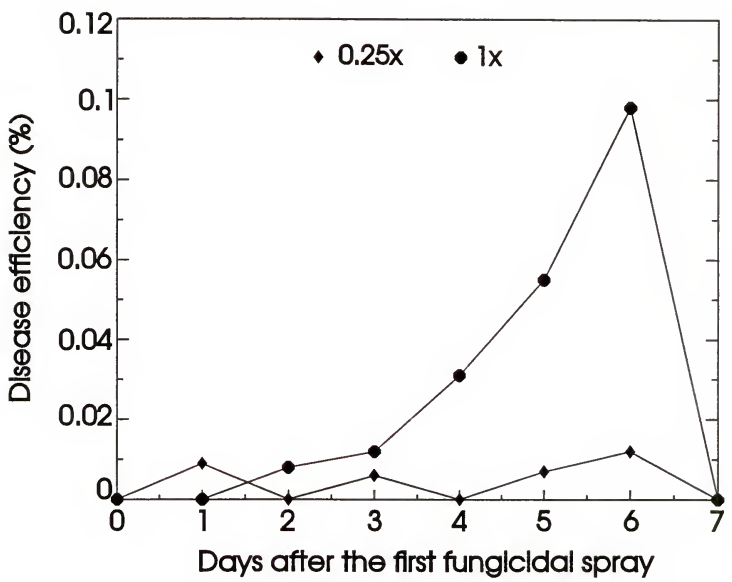

Figure 3.3. Disease efficiency of Puccinia coronata f.sp. avenae on oats plants sprayed with $0.25 \times$ and $1 \times$ the label rate of mancozeb in the greenhouse in 1995. Fungicidal rates were applied to independent sets of plants at different times prior to inoculation. Oat plants were inoculated once with $2 \times 10^{4}$ uredospores $\mathrm{ml}^{-1}$. The disease efficiency of the crown rust fungus was $4.05 \%$ on nonsprayed plants. 


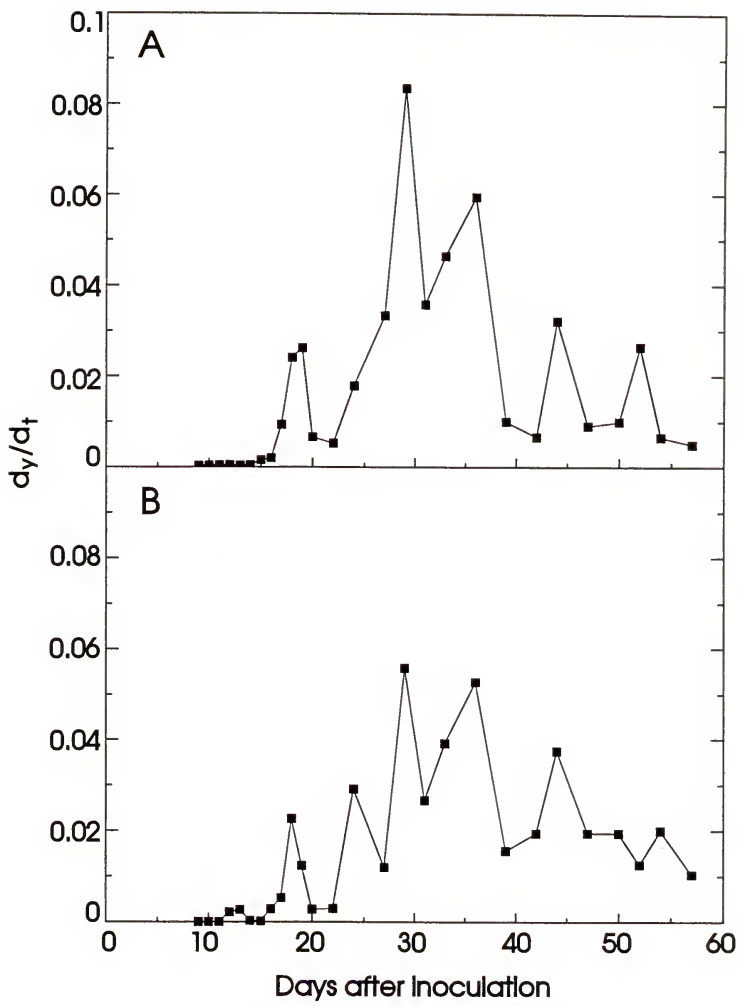

Figure 3.4. Estimated daily increment $\left(d_{y} / d_{t}\right)$ of crown rust in nonsprayed oats in the field in 1995. Experiments were planted on 19 November 1994 (A) and 3 December 1994 (B) and inoculated on 10 February 1995. 
rust severity occurred around day 29 , that is, about three latent periods after inoculation. Only at this time was $d_{y} / d_{t}$ higher in experiment A (0.083) than in B (0.056).

On nonsprayed plots, crown rust completely destroyed the plants in 2 months. In 1995-A, the disease progressed from $0.000152\left(y_{0}\right)$ to $0.981\left(y_{f}\right)$ in 48 days (Fig. 3.5-A). The epidemic rate $\left(r_{G}\right)$, calculated by the two-point method (Berger, 1988), was 0.127 gompits day ${ }^{-1}$. In 1995-B, $y_{0}, y_{f}$ and $r_{G}$ were $0.000187,0.946$, and 0.105 gompits day ${ }^{-1}$, respectively (Fig. 3.5-B). The area under the disease progress curve (AUDPC) was 23.54 in $\mathrm{A}$ and 20.03 in B.

Transformation of disease proportions into gompits allowed the differentiation of two disease waves for each epidemic of crown rust on oats (Fig. 3.6-A and 3.6-B). The first wave occurred from 9 to 15 days and the second epidemic wave from 16 to 22 days after inoculation of the rust fungus. In 1995-A, crown rust increased 26-fold during the first and 21-fold during the second epidemic wave. The disease increase in 1995-B was higher (30-fold) during the first but lower (11-fold) during the second wave. After the second epidemic wave, the increase in crown rust in 1995-B (Fig. 3.6-B) was nearly a straight line. In 1995-A (Fig. 3.6-A), however, some wave-like disease progress was observed to the end of the epidemic.

In the experiment conducted in 1996, low temperatures recorded in February and March restricted the development of crown rust epidemics to only 21 days. The initial and final disease proportions observed on nonsprayed plants were 0.001 and 0.15 , which resulted in an estimated epidemic rate $\left(r_{G N L}\right)$ of 0.048 gompits day ${ }^{-1}$. Only one epidemic wave, whose breadth was about 14 days, was observed for nonsprayed plants and most fungicidal spray programs (Fig. 3.7). 


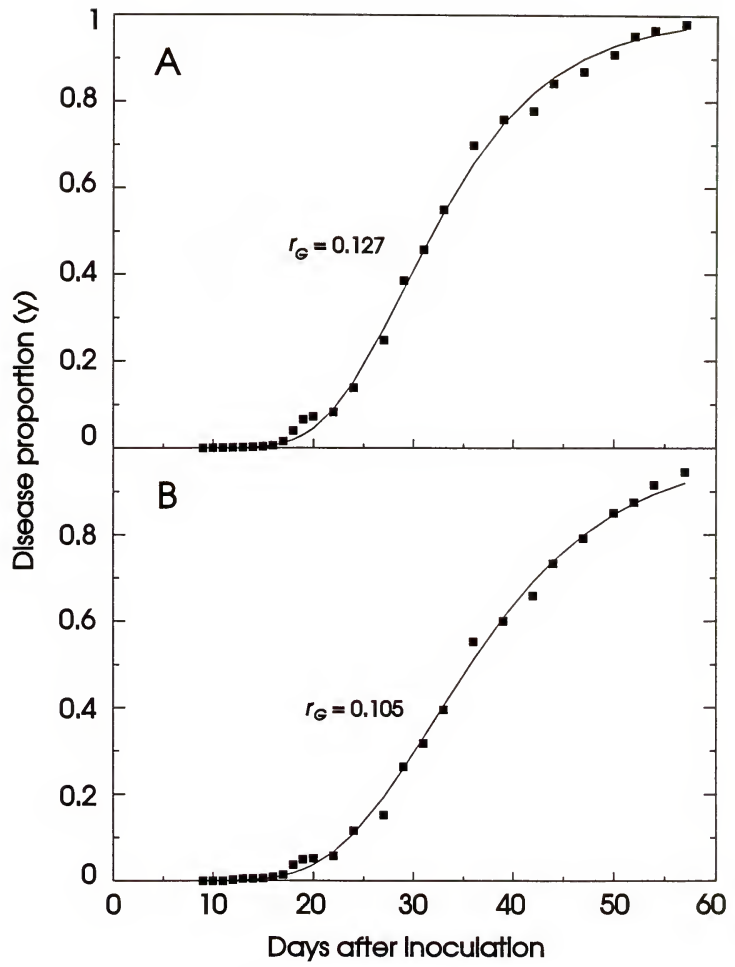

Figure 3.5. Progress of crown rust in nonsprayed oats in the field in 1995. Experiments were planted on 19 November 1994 (A) and 3 December 1994 (B) and inoculated on 10 February 1995. The epidemic rates $\left(r_{G}\right)$ were calculated as [gompit $\left(y_{f}\right)$ - gompit $\left(y_{0}\right)$ ] / $\Delta t$, where gompit $=-\ln [-\ln (y)], y_{f}=$ final disease proportion, $y_{0}=$ initial disease proportion, and $\Delta t=$ time interval between $t_{0}$ and $t_{f}$. Points on the graph are original data. The line was generated by nonlinear regression according to the Gompertz model. 


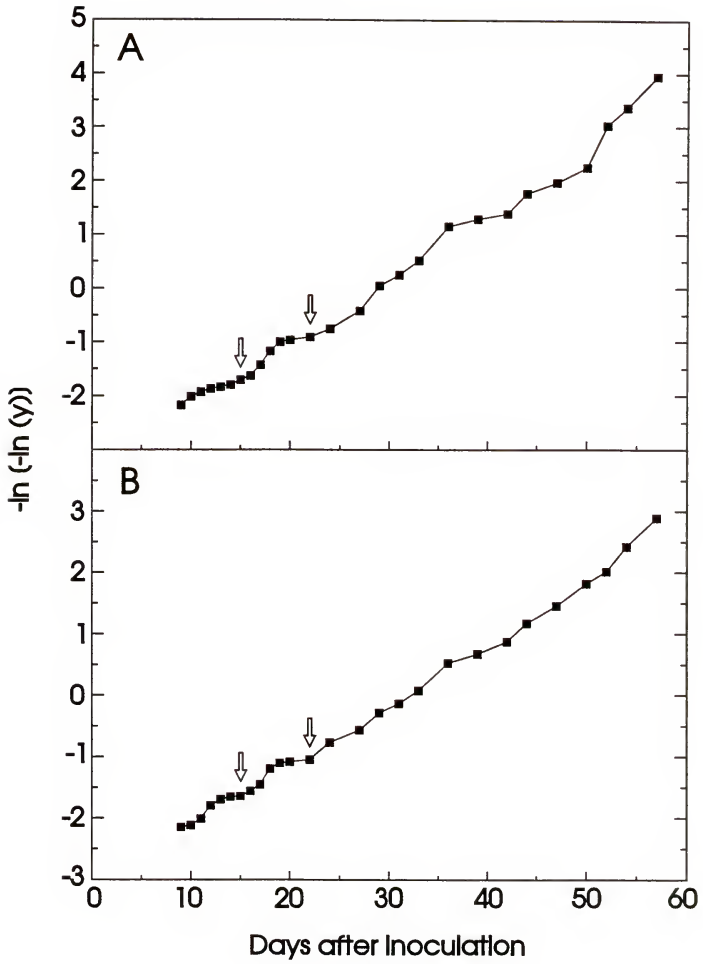

Figure 3.6. Epidemic waves for crown rust in nonsprayed oats in the field in 1995 . Experiments were planted on 19 November 1994 (A) and 3 December 1994 (B) and inoculated on 10 February 1995 . The disease proportions $(y)$ were transformed by $-\ln [-\ln$ $(y)]$ to show the epidemic waves, whose ends are indicated by arrows. 
Figure 3.7. Progress of crown rust in nonsprayed oats in the field in 1996. The epidemic rate $\left(r_{G N L}\right)$ was estimated by nonlinear regression of disease proportions against time using the Gompertz model. The disease proportions $(y)(\mathrm{A})$ were transformed by $-\ln [-\ln$ $(y)]$ to show the epidemic waves (B), whose ends are indicated by arrows. This experiment was planted on 2 December 1995. 


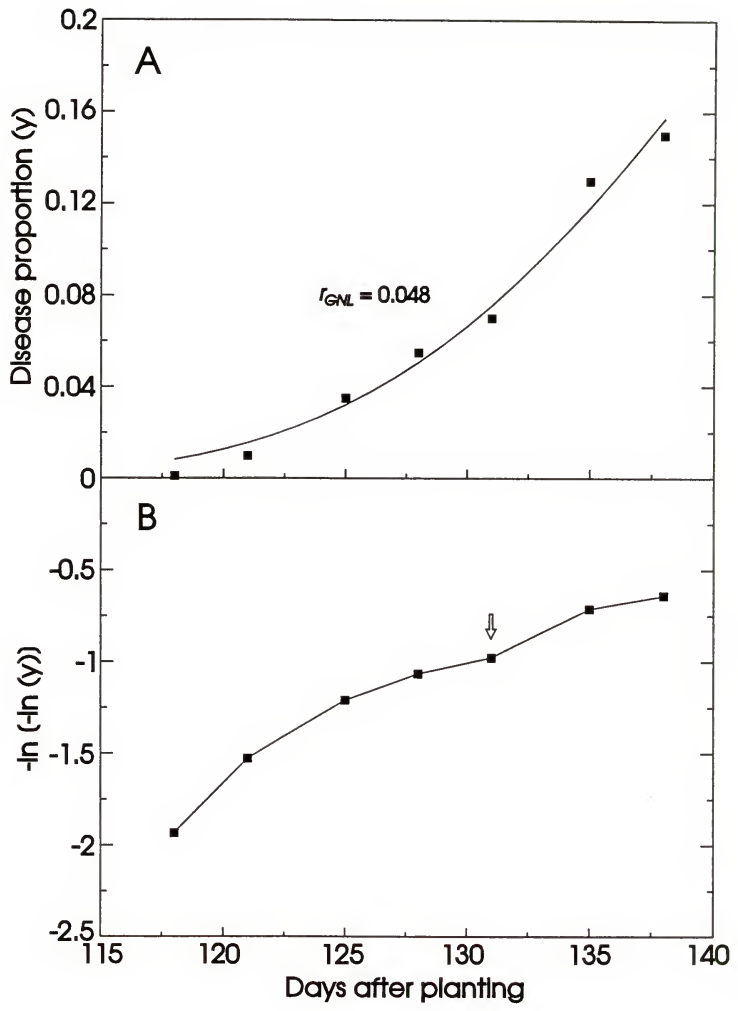


In 1997, the crown rust epidemic on nonsprayed plants was as severe as those assessed in the 1995 experiments. The first rust pustules were observed on 25 February 1997, that is, 10 days after artificial inoculation of the oat plants. Crown rust progressed from $0.0001\left(y_{0}\right)$ to $0.96\left(y_{f}\right)$ at an estimated epidemic rate $\left(r_{G N L}\right)$ of 0.134 gompits day ${ }^{-1}$ (Fig. 3.8). The daily increments of disease severity $\left(d_{y} / d_{t}\right)$ (Fig. 3.8) increased to a maximum of 0.055 on day 34 and declined afterward. Again in 1997, two epidemic waves were distinguished when disease values transformed by gompits were plotted over time (Fig. 3.9). Both epidemic waves had similar breadth, which was 7-8 days long. Oat crown rust increased 30 -fold during the first wave but only four-fold during the second epidemic wave.

\section{Effect of Reduced Rates of Fungicide on Epidemics of Crown Rust of Oats}

Applications of the fungicide mancozeb at either $1 \times$ or $0.25 \times$ of its label rate reduced the initial amount of crown rust significantly $(P=0.05)$ (Table 3.1). In the 1995A experiment, $y_{0}$ decreased from 0.000152 on nonsprayed plants to 0.00001 on the $1 \times$ treatment and to 0.000005 on the $0.25 \times$ treatment. In $1995-\mathrm{B}, y_{0}$ ranged from 0.000003 ( $1 \times$ and $0.25 \times$ ) to 0.000187 (control). No significant differences in $y_{0}$ were observed between $1 \times$ and $0.25 \times$ rates in both experiments. The $y_{0}$ measured in the sprayed plots $(0.25 \times$ and $1 \times)$ referred to the amount of disease associated with the inoculation carried out 9 days before.

In addition to the reduction on the amount of initial disease, sprays of the lower fungicidal rate (0.25x) delayed epidemic onset (Fig. 3.10). In both 1995-A and 1995-B experiments, sprays of $0.25 \times$ rate maintained disease severity constant for 7 days, that is, 


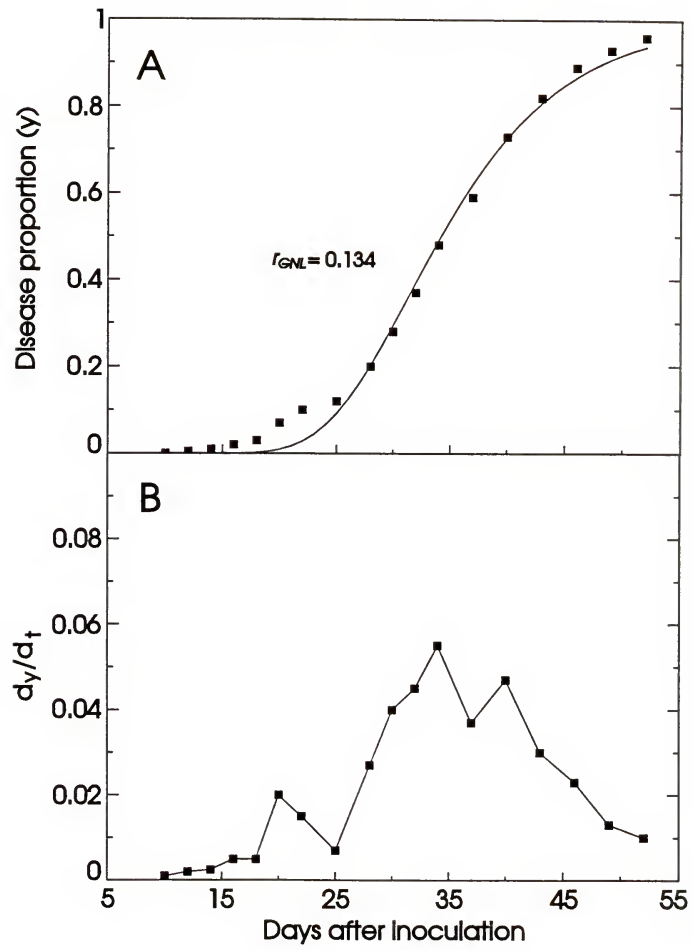

Figure 3.8. Disease progress curve (A) and estimated daily increment of disease severity $\left(d_{y} / d_{t}\right)$ (B) for crown rust in nonsprayed oats in 1997. The epidemic rate $\left(r_{G N L}\right)$ was estimated by nonlinear regression of disease proportions against time using the Gompertz model. The oat plants were inoculated on 15 February 1997. 


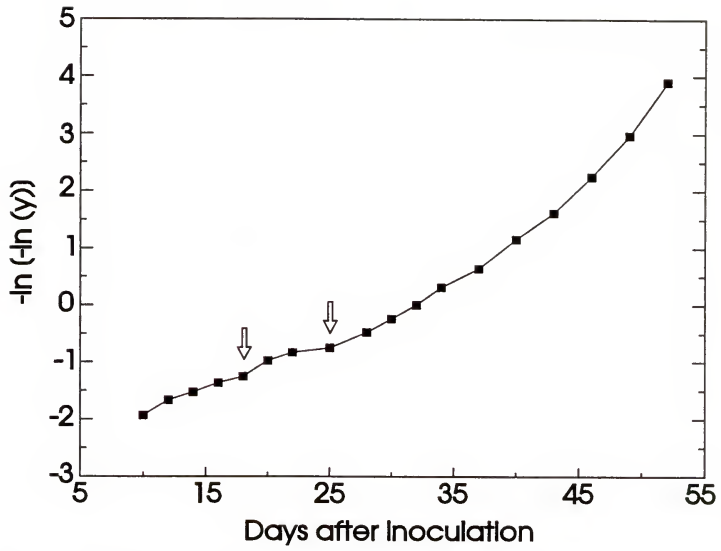

Figure 3.9. Epidemic waves for crown rust in nonsprayed oats in 1997. The disease proportions $(y)$ were transformed by $-\ln [-\ln (y)]$ to show the epidemic waves, whose ends are indicated by arrows. The oat plants were inoculated on 15 February 1997. 
Figure 3.10. Epidemic waves for oat crown rust under reduced- and full-rate programs of fungicidal sprays in 1995 . The reduced $(0.25 \times)$ and the full $(1 \times)$ rates of mancozeb were applied 22 (three times a week) and seven times (once a week) after inoculation. The experiments were planted on 19 November 1994 (A) and 3 December 1994 (B) and inoculated on 10 February 1995 . The disease proportions $(y)$ were transformed by $-\ln [-\ln$ $(y)]$ to show the epidemic waves, whose ends are indicated by arrows. 


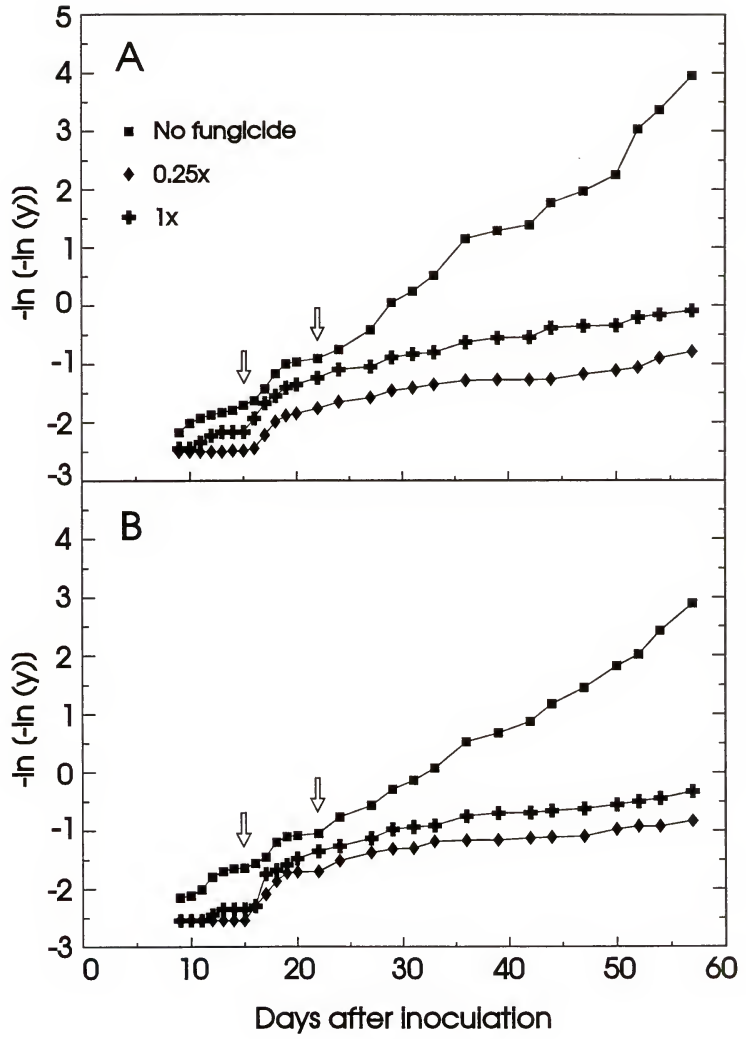


no infection of the test plants occurred during that period. Under the same conditions, the full rate $(1 \times)$ delayed epidemic onset only $2-3$ days.

Table 3.1: Initial $\left(y_{0}\right)$ and final $\left(y_{f}\right)$ proportions of disease, epidemic rates $\left(r_{G}\right)$, and areas under the disease progress curves (AUDPC) for epidemics of oat crown rust under sprays of $1 \times$ (weekly) or $0.25 \times$ (three times a week) the label rate of mancozeb. The proportions of disease severity were assessed on flag, $\mathrm{f}-1, \mathrm{f}-2$, and $\mathrm{f}-3$ leaves. Experiments conducted in 1995

\begin{tabular}{|c|c|c|c|c|}
\hline \multirow{2}{*}{$\begin{array}{l}\text { Fungicidal } \\
\text { spray program }\end{array}$} & \multicolumn{4}{|c|}{ Epidemic parameter } \\
\hline & $y_{0}$ & $y_{f}$ & $\boldsymbol{r}_{G}^{\mathrm{y}}$ & AUDPC \\
\hline \multicolumn{5}{|l|}{ Experiment A } \\
\hline $0.25 x$ & $0.000005 \mathrm{a}^{\mathrm{z}}$ & $0.11 \mathrm{a}$ & $0.036 \mathrm{a}$ & $1.18 \mathrm{a}$ \\
\hline $1 \times$ & $0.00001 \mathrm{a}$ & $0.335 \mathrm{~b}$ & $0.047 \mathrm{~b}$ & $6.28 \mathrm{~b}$ \\
\hline Nonsprayed & $0.000152 \mathrm{~b}$ & $0.981 \quad \mathrm{c}$ & $0.127 \quad \mathrm{c}$ & $23.54 \mathrm{c}$ \\
\hline \multicolumn{5}{|l|}{ Experiment B } \\
\hline $0.25 x$ & $0.0000003 \mathrm{a}$ & $0.1 \quad \mathrm{a}$ & $0.039 \mathrm{a}$ & $1.57 \mathrm{a}$ \\
\hline $1 \times$ & $0.0000003 \mathrm{a}$ & $0.25 \quad \mathrm{~b}$ & $0.05 \quad \mathrm{~b}$ & $4.4 \quad \mathrm{~b}$ \\
\hline Nonsprayed & $0.000187 \quad b$ & $0.946 \mathrm{c}$ & $0.105 \mathrm{c}$ & $20.03 \mathrm{c}$ \\
\hline $\mathrm{P}>\mathrm{F}($ Exp. A) & 0.001 & 0.0001 & 0.001 & 0.0001 \\
\hline $\mathrm{P}>\mathrm{F}(\operatorname{Exp} . \mathrm{B})$ & 0.0001 & 0.0001 & 0.002 & 0.0001 \\
\hline
\end{tabular}

${ }^{y}$ Epidemic rates were calculated as [gompit $\left(y_{f}\right)$ - gompit $\left.\left(y_{0}\right)\right] / \Delta t$, where gompit $=-\ln [-\ln (v)], y_{f}$ $=$ final disease proportion, $y_{0}=$ initial disease proportion, and $\Delta t=$ time interval between $t_{0}$ and $t_{\text {s. }}$

${ }^{2}$ Treatment means within columns followed by the same letter do not differ significantly $(P<$ 0.05 ) by Duncan's Multiple Range Test, using the variability pooled among replicates. Even though rate of fungicide is a quantitative factor, the individual rates were applied at different intervals and therefore the treatments were considered unique.

Applications of fungicide also slowed the epidemic rate of crown rust and reduced the amount of disease at the end of the epidemic (Table 3.1 and Fig. 3.11). For example, the epidemic rate in 1995 -A was 0.127 for nonsprayed plants, 0.047 for the $1 \times$ rate, and 0.036 gompits day ${ }^{-1}$ for the $0.25 \times$ rate. The final proportion of disease severity was reduced from 0.98 on unsprayed plants to as low as 0.11 on the $0.25 \times$-spray treatment. The AUDPC measured on plants sprayed with the $0.25 \times$ rate $(1.18)$ was very low 
Figure 3.11. Disease progress curves for epidemics of crown rust subjected to reducedand full-rate programs of fungicidal sprays in 1995 . The reduced $(0.25 \times)$ and the full $(1 \times)$ rates of mancozeb were applied 22 (three times a week) and seven times (once a week) after inoculation. Experiments were planted on 19 November 1994 (A) and 3 December 1994 (B) and inoculated on 10 February 1995. The epidemic rates $\left(r_{G}\right)$ were calculated as [gompit $\left(y_{f}\right)$ - gompit $\left.\left(y_{0}\right)\right] / \Delta t$, where gompit $=-\ln [-\ln (y)], y_{f}=$ final disease proportion, $y_{0}=$ initial disease proportion, and $\Delta t=$ time interval between $t_{0}$ and $t_{f}$. Points on the graph are original data. The lines were generated by nonlinear regression according to the Gompertz model. 


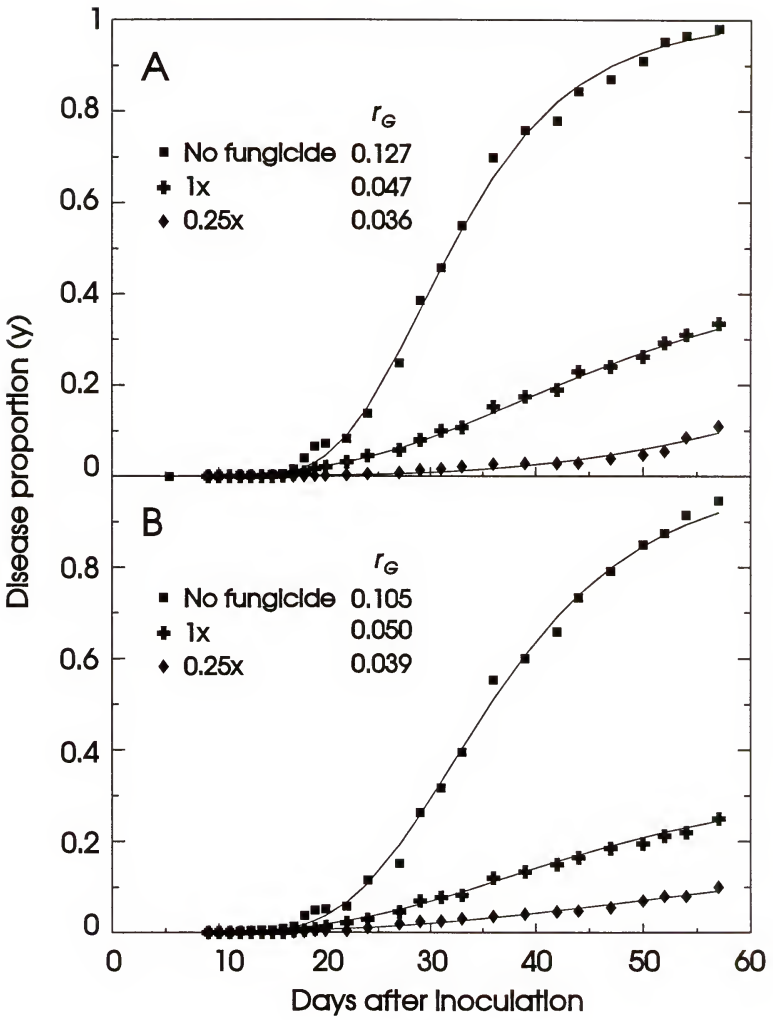


compared to the AUDPC of unsprayed plants (23.54). Overall, the $0.25 \times$-fungicidal schedule always had lower $r_{G}, y_{f}$, and AUDPC than the $1 \times$-spray treatment.

The kernel yield obtained from plants sprayed with fungicide was $94 \%$ ( $1 \times$ rate) to $110 \%(0.25 \times$ rate $)$ higher than that from nonsprayed controls (Table 3.2$)$. Fungicidal applications also improved the thousand-kernel weight significantly $(P=0.05)$ over the nonsprayed plants. Differences in kemel yield between the $0.25 \times$ and $1 \times$ rates were observed in the experiment $\mathrm{A}$, in which the epidemic of crown rust was more severe than in the experiment $\mathrm{B}$.

Table 3.2: Kernel yields of oats subjected to sprays of $0.25 \times$ (three times a week) or $1 \times$ mancozeb (once a week) to control crown rust in two experiments (A and B) on oats in 1995

\begin{tabular}{|c|c|c|c|c|c|c|}
\hline \multirow{2}{*}{$\begin{array}{l}\text { Fungicidal } \\
\text { spray program }\end{array}$} & \multicolumn{3}{|c|}{ Kernel yield $\left(\mathrm{kg} \mathrm{ha}^{-1}\right)$} & \multicolumn{3}{|c|}{ Thousand-kernel weight (g) } \\
\hline & $\mathbf{A}$ & B & Mean & $\mathbf{A}$ & B & Mean \\
\hline $0.25 x$ & $5975 \mathrm{a}^{2}$ & $5550 \mathrm{a}$ & 5762.5 & $33.6 \mathrm{a}$ & $33.8 \mathrm{a}$ & 33.7 \\
\hline $1 \times$ & $5250 \mathrm{~b}$ & $5380 \mathrm{a}$ & 5315.0 & $34.0 \mathrm{a}$ & $32.2 \mathrm{a}$ & 33.1 \\
\hline Nonsprayed & $2323 \mathrm{c}$ & $3160 \mathrm{~b}$ & 2741.5 & $28.7 \mathrm{~b}$ & $27.4 \mathrm{~b}$ & 28.1 \\
\hline $\mathrm{P}>\mathrm{F}$ & 0.0001 & 0.0001 & & 0.0002 & 0.0002 & \\
\hline
\end{tabular}

${ }^{\mathrm{z}}$ Treatment means within columns followed by the same letter do not differ significantly $(P<$ 0.05 ) by Duncan's Multiple Range Test. Even though rate of fungicide is a quantitative factor, the individual rates were applied at different intervals and therefore the treatments were considered unique.

In 1996, although there was an overall low amount of disease, significant differences in $y_{0}, y_{f}$, and AUDPC were observed among spray programs (Table 3.3 and Fig. 3.12). The $1 \times$ rate of mancozeb reduced $y_{0}$ from 0.001 to 0.0001 , but only the $0.5 \times$ and $0.25 \times$ rates delayed epidemic onset 7 and 10 days, respectively. The reduced fungicidal rates had also $y_{f}$ and AUDPC lower than the $1 \times$ rate. The $0.5 \times$ and $0.25 \times$ rates 
Figure 3.12. Disease progress curves (A) and epidemic waves (B) for crown rust of oats under reduced- and full-rate programs of fungicidal sprays in 1996 . The $0.25 \times$ and $0.5 \times$ rates of mancozeb were applied at twice-weekly (seven sprays) and weekly intervals (four sprays) after inoculation. The standard $1 \times$ treatment was applied twice at 109 and 120 days after planting. The epidemic rates $\left(r_{G N L}\right)$ were estimated by nonlinear regression of disease proportions against time using the Gompertz model. The disease proportions $(y)$ were transformed by $-\ln [-\ln (y)]$ to show the epidemic waves, whose ends are indicated by arrows. This experiment was planted on 2 December 1995. 


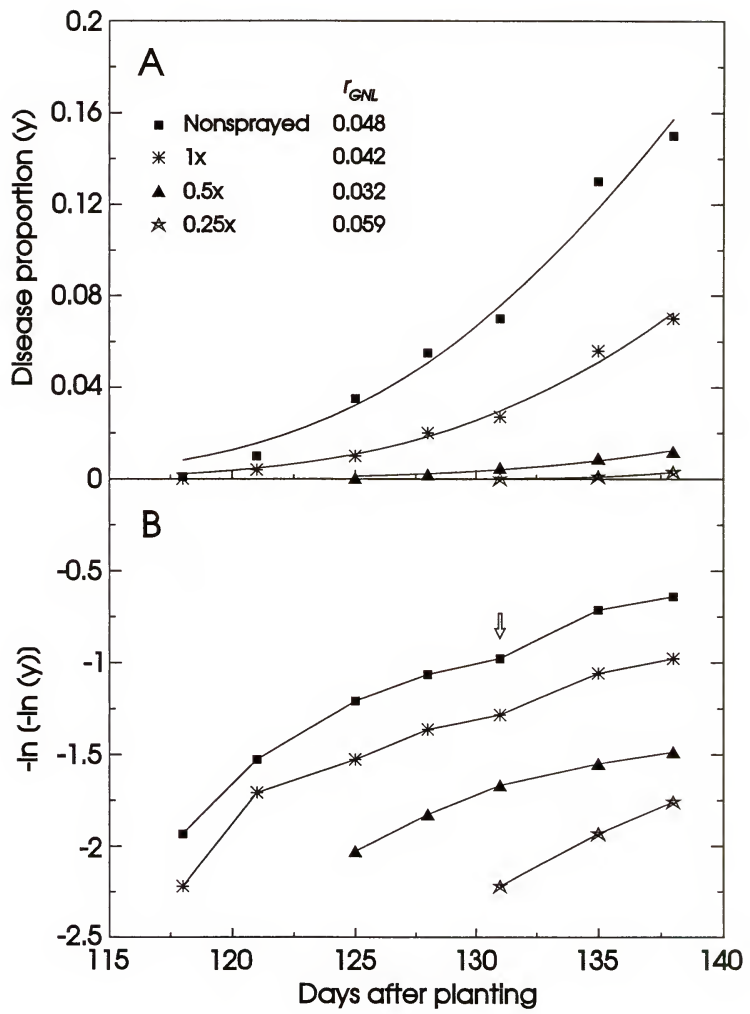


did not differ from each other except on epidemic onset. The low and late occurrence of crown rust in this experiment did not affect oat yield significantly $(P=0.96)$.

Table 3.3: Initial $\left(y_{0}\right)$ and final $\left(y_{f}\right)$ proportions of disease, estimated epidemic rates $\left(r_{G N L}\right)$, areas under the disease progress curves (AUDPC), and oat yields associated with epidemics of crown rust subjected to biweekly $(1 \times)$, weekly $(0.5 \times)$, or twice-weekly ( $0.25 \times$ rate) sprays of mancozeb in 1996 . The proportions of disease severity were assessed on flag, $f-1, f-2$, and $f-3$ leaves

\begin{tabular}{|c|c|c|c|c|c|}
\hline \multirow{2}{*}{$\begin{array}{l}\text { Fungicidal } \\
\text { spray program }\end{array}$} & \multicolumn{4}{|c|}{ Epidemic parameter } & \multirow{2}{*}{$\begin{array}{l}\text { Yield } \\
\left(\mathrm{kg} \mathrm{ha}^{-1}\right)\end{array}$} \\
\hline & $y_{0}{ }^{x}$ & $y_{f}$ & $\boldsymbol{r}_{G N L}^{\mathrm{y}}$ & AUDPC & \\
\hline $0.25 x$ & 0.0001 & $0.003 \mathrm{a}^{\mathrm{z}}$ & 0.059 & $0.01 \mathrm{a}$ & 5328 \\
\hline $0.5 x$ & 0.0005 & $0.012 \mathrm{a}$ & 0.032 & $0.07 \mathrm{a}$ & 5331 \\
\hline $1 \times$ & 0.0001 & $0.070 \mathrm{~b}$ & 0.042 & $0.50 \mathrm{~b}$ & 5251 \\
\hline Nonsprayed & 0.0010 & $0.150 \quad \mathrm{c}$ & 0.048 & $1.25 \mathrm{c}$ & 5113 \\
\hline $\mathrm{P}>\mathrm{F}$ & & 0.0001 & 0.125 & 0.0001 & 0.96 \\
\hline
\end{tabular}

${ }^{x}$ Due to epidemic delays, $y_{0}$ was measured at 118 (control and $\left.1 \times\right), 125(0.5 x)$, and $131(0.25 x)$ days after planting. Data for $y_{0}$ were not submitted to statistical analysis.

${ }^{y}$ Epidemic rates were estimated by nonlinear regression of disease proportions against time using the Gompertz model.

z Treatment means within columns followed by the same letter do not differ significantly $(P<$ 0.05 ) by Duncan's Multiple Range Test, using the variability pooled among replicates. Even though rate of fungicide is a quantitative factor, the individual rates were applied at different intervals and therefore the treatments were considered unique.

Applications of mancozeb according to various spray programs in 1997 resulted in differential effects on the amount of initial disease $\left(y_{0}\right)$ (Table 3.4 and Fig. 3.13). The standard treatment, based on three applications of $1 \times$ mancozeb at 10- to 12-day intervals from growth stage 32 ( 7 in the Feekes scale), did not reduce $y_{0}$ because the fungicide was initially applied to plants already diseased (0.001). This resulted in similar epidemic waves (Fig. 3.13) between the standard treatment and the unsprayed control. Reduction of the fungicidal rate to $0.5 \times$, anticipation of the first spray to the day of inoculation, and shortening of the spray interval to 1 week decreased $y_{0} 10$-fold, but it had no effect on 


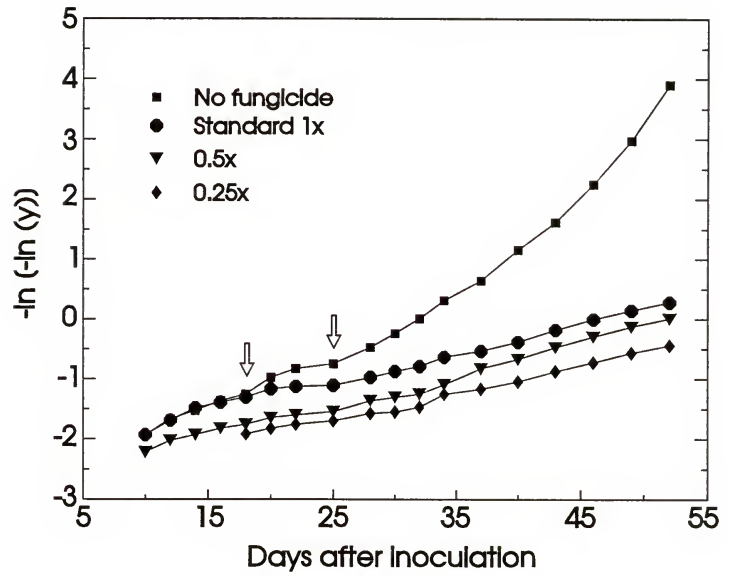

Figure 3.13. Epidemic waves for crown rust of oats under reduced- and full-rate programs of fungicidal sprays in 1997 . The fungicide mancozeb was applied at semi-weekly (12 sprays at the $0.25 \times$ rate) or weekly intervals (six sprays at the $0.5 \times$ rate) after inoculation. The standard $1 \times$ treatment was sprayed three times at 14,25 , and 37 days after inoculation. The disease proportions $(y)$ were transformed by $-\ln [-\ln (y)]$ to show the epidemic waves, whose ends are indicated by arrows. The oat plants were inoculated on 15 February 1997. 
epidemic onset. Applications of $0.25 \times$ mancozeb twice a week from the inoculation (note: in 1995 , the $0.25 \times$ rate was applied three times a week) provided complete control of crown rust and delayed epidemic onset one latent period (about 8 days).

All fungicidal spray programs slowed the estimated epidemic rate $\left(r_{G N L}\right)$ of crown rust significantly $(P=0.05)$ (Table 3.4). However, no differences in $r_{G N L}$ were observed among the $0.25 \times, 0.5 \times$, and $1 \times$ rates of the fungicide mancozeb.

Table 3.4: Initial $\left(y_{0}\right)$ and final $\left(y_{f}\right)$ proportions of disease, estimated epidemic rates $\left(r_{G N L}\right)$, and areas under the disease progress curves (AUDPC) for epidemics of oat crown rust under various programs of fungicidal sprays in 1997. The $0.25 \times, 0.5 \times$, and $1 \times$ rates of the fungicide mancozeb were applied at 3-4-, 7-, and 10-day intervals, in a total of 12 , seven, and three sprays, respectively. The proportions of disease severity were assessed on flag, $f-1, f-2$, and $f-3$ leaves

\begin{tabular}{|c|c|c|c|c|}
\hline \multirow{2}{*}{$\begin{array}{l}\text { Fungicidal } \\
\text { spray program }\end{array}$} & \multicolumn{4}{|c|}{ Epidemic parameter } \\
\hline & $y_{0}$ & $y_{f}$ & $r_{G N L}{ }^{x}$ & AUDPC \\
\hline $0.25 x$ & $0.0011^{y}$ & $0.21 \mathrm{a}^{\mathrm{z}}$ & $0.042 \mathrm{a}$ & $1.93 \mathrm{a}$ \\
\hline $0.5 x$ & $0.0001 \mathrm{a}$ & $0.37 \mathrm{~b}$ & $0.051 \mathrm{a}$ & $3.97 \mathrm{~b}$ \\
\hline Standard $1 \times$ & 0.001 & $0.47 \mathrm{c}$ & $0.052 \mathrm{a}$ & 6.6 \\
\hline Nonsprayed & 0.001 & 0.96 & $0.134 \mathrm{~b}$ & 17.2 \\
\hline$P>F$ & 0.003 & 0.0005 & 0.01 & 0.0001 \\
\hline \multicolumn{5}{|c|}{$\begin{array}{l}{ }^{\text {}} \text { Epidemic rates were estimated by nonlinear regression of disease proportions against time using } \\
\text { the Gompertz model. } \\
\text { y In the } 0.25 \times \text { treatment, the crown rust epidemic initiated } 7 \text { days later than in the other spray } \\
\text { programs. Therefore, this treatment was not included in the statistical analysis of } y_{0} \text {. } \\
\text { a Treatment means within columns followed by the same letter do not differ significantly }(P<< \\
0.05 \text { ) by Duncan's Multiple Range Test, using the variability pooled among replicates. Even } \\
\text { though rate of fungicide is a quantitative factor, the individual rates were applied at different } \\
\text { intervals and therefore the treatments were considered unique. }\end{array}$} \\
\hline
\end{tabular}

The final amount of disease $\left(y_{f}\right)$ and the AUDPC were greatly reduced by all three fungicidal spray programs (Table 3.4 and Fig. 3.14). The control of crown rust was greater as the fungicidal rates were reduced and applied earlier and more often. For example, plants sprayed with the $0.25 \times$ rate had the lowest $y_{f}$ and AUDPC. Although the 


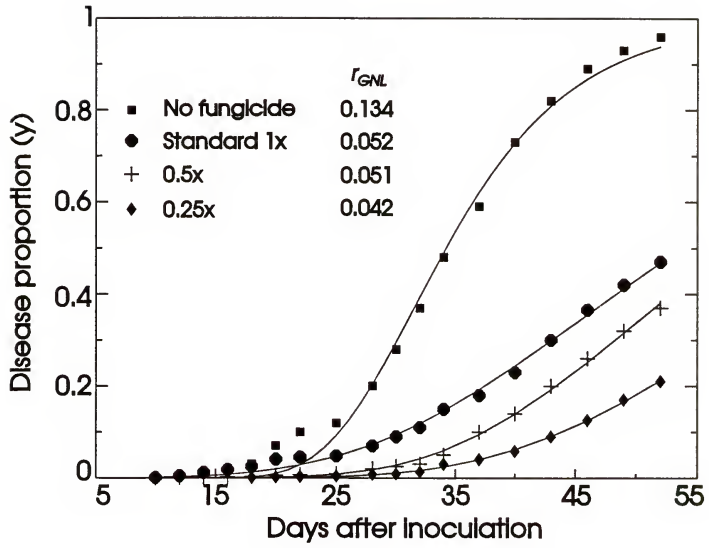

Figure 3.14. Disease progress curves for crown rust of oats under reduced- and full-rate programs of fungicidal sprays in 1997. The fungicide mancozeb was applied at semiweekly (12 sprays at the $0.25 \times$ rate) or weekly intervals (six sprays at the $0.5 \times$ rate) after inoculation. The standard $1 \times$ treatment was sprayed three times at 14,25 , and 37 days after inoculation. The epidemic rates $\left(r_{G N L}\right)$ were estimated by nonlinear regression of disease proportions against time using the Gompertz model. The oat plants were inoculated on 15 February 1997. 
epidemic rates for the $1 \times-, 0.5 \times-$, and $0.25 \times$-spray programs did not differ, the low-rate schedules resulted in significantly $(P=0.05)$ less disease. This better disease control was attributed to the reduction on $y_{0}$ provided by the $0.5 \times$ schedule and the delay on epidemic onset associated with preventive applications of the $0.25 \times$ rate of fungicide.

Crown rust of oats severely affected the yield and the quality of oat kernels in 1997 (Table 3.5). The kernel yield of non-sprayed plants $\left(1532 \mathrm{~kg} \mathrm{ha}^{-1}\right)$ was less than half that quantified for the standard $1 \times$ treatment $\left(3213 \mathrm{~kg} \mathrm{ha}^{-1}\right)$. Application of the $0.5 \times$ and $0.25 \times$ fungicidal rates at short spray intervals increased yield significantly $(P=0.05)$ compared to the $1 \times$ rate. The thousand-kernel weight obtained from plants sprayed with $0.25 \times$ mancozeb twice a week was the highest among all treatments.

Table 3.5: Kernel yields and thousand-kernel weights of oat plants subjected to various fungicidal spray programs to control crown rust in 1997 . The $0.25 \times, 0.5 \times$, and $1 \times$ rates of the fungicide mancozeb were applied at 3-4-, 7-, and 10-day intervals, in a total of 12 , seven, and three fungicidal sprays, respectively

\begin{tabular}{lcc}
\hline Fungicidal spray program & Yield $\left(\mathbf{k g ~ h a}^{-\mathbf{1}}\right)$ & Thousand-kernel weight (g) \\
\hline $0.25 \times$ & $4139 \mathrm{a}^{\mathrm{z}}$ & $28.6 \mathrm{a}$ \\
$0.5 \times$ & $3771 \mathrm{a}$ & $24.8 \mathrm{~b}$ \\
Standard 1× & $3213 \mathrm{~b}$ & $24.2 \mathrm{~b}$ \\
Nonsprayed & $1532 \mathrm{c}$ & $20.8 \mathrm{c}$ \\
\hline P $>$ F & 0.0001 & 0.0001 \\
\hline
\end{tabular}

${ }^{z}$ Treatment means within columns followed by the same letter do not differ significantly $(P<$ 0.05 ) by Duncan's Multiple Range Test. Even though rate of fungicide is a quantitative factor, the individual rates were applied at different intervals and therefore the treatments were considered unique.

\section{Effect of Action Thresholds on the Management of Crown Rust of Oats with Fungicide}

In 1995 , the $0.0001,0.001,0.01$, and 0.05 action thresholds were detected at 9 , 12,17 , and 19 days after inoculation of the field plots. The first and the second action 
thresholds ( 0.0001 and 0.001$)$ occurred at the beginning and middle of the first epidemic wave, respectively. The third and fourth thresholds $(0.01$ and 0.05$)$ were observed during the second epidemic wave. Although crown rust was assessed daily in the early stages of the epidemic, precise determination of action thresholds was complicated by the rapid progress of the epidemic. For instance, the disease severity increased 11-fold (from 0.006 to 0.066$)$ between days 16 and 19 , thus comprising two thresholds $(0.01$ and 0.05$)$ in a 3day period.

Applications of fungicide according to action thresholds did not affect the epidemic waves of crown rust. No delay on epidemic onset nor reduction on the amount of initial disease was observed among the various threshold treatments. Indeed, all threshold treatments, no matter what the fungicidal rate, had epidemic waves with similar shape and intensity to that of unsprayed plants (Fig. 3.15-A and 3.15-B).

Initiation of fungicidal sprays at higher disease severities resulted in increased epidemic rates (Table 3.6 and Fig. 3.16). Among the $0.25 \times$-fungicidal schedules, the epidemic rate $\left(r_{G}\right)$ for action thresholds between 0 and 0.01 increased from 0.036 to 0.058 gompits day ${ }^{-1}$. The relationship of $r_{G}$ to the amount of disease at the time fungicidal sprays were initiated $\left(y_{t l d}\right)$ (Fig. 3.17) was represented by the quadratic equation $Y_{0.25 x}=$ $0.034-0.0357 X-0.0134 X^{2}, R^{2}=0.99$, in which $Y=r_{G}$ and $X=$ gompit of $y_{t d d^{*}}$ In the $1 \times-$ spray schedules, $r_{G}$ ranged from 0.049 to 0.063 gompits day $^{-1}$ for $y_{t l d}$ between 0.0001 and 0.01. No effect on $r_{G}$ was observed for $y_{t d}$ between the thresholds of 0 and 0.0001 , and between 0.01 and 0.05 . The relationship of $r_{G}$ to $y_{t d d}$ was represented by the cubic equation $Y_{1 \times}=-0.039-0.198 X-0.119 X^{2}-0.021 X^{3}, R^{2}=1.00$, where $Y$ and $X$ were as defined above. 
Figure 3.15. Disease waves for epidemics of oat crown rust subjected to fungicidal spray programs initiated at the $0,0.0001,0.001,0.01$, and 0.05 action thresholds in 1995. The $0.25 \times$ rate of mancozeb was applied three times a week in a total of $22(0), 18(0.0001)$, $17(0.001), 15(0.01)$, or 14 sprays $(0.05)$. The $1 \times$ fungicidal rate was sprayed weekly for seven (0), six (0.0001, and 0.001$)$, or five times $(0.01$ and 0.05$)$. The disease proportions (y) were transformed by $-\ln [-\ln (y)]$ to show the epidemic waves, whose ends are indicated by arrows. The oat plants were inoculated on 10 February 1995. 


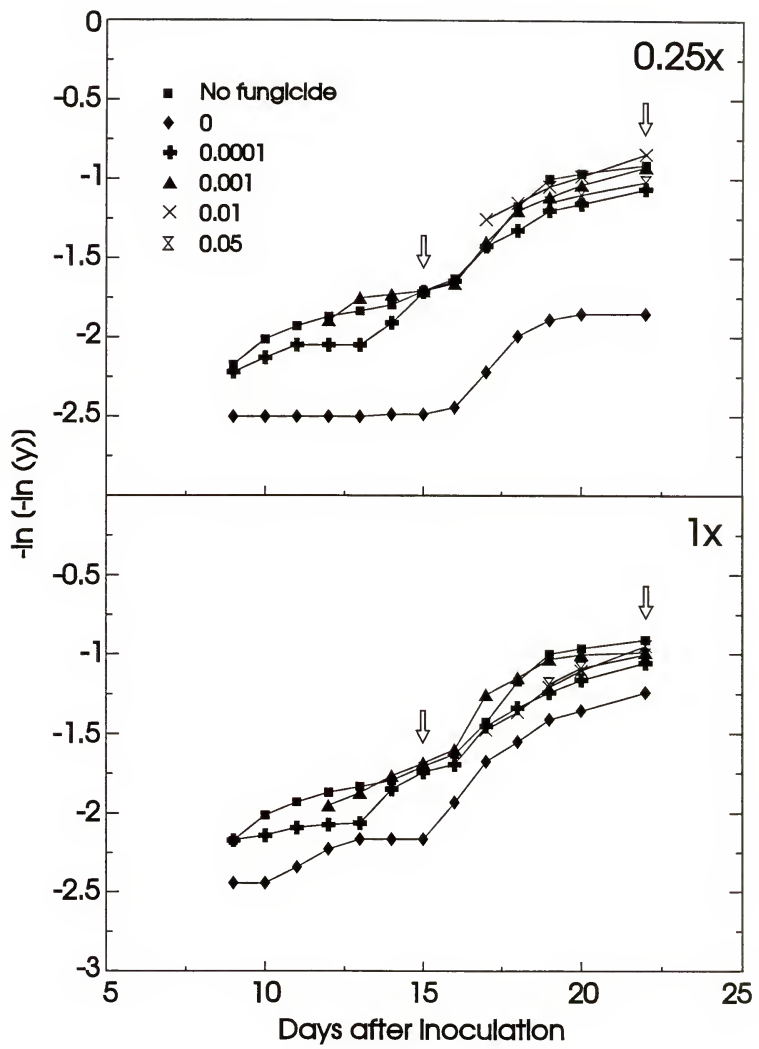


Figure 3.16. Disease progress curves for crown rust epidemics subjected to fungicidal spray programs initiated at the $0,0.0001,0.001,0.01$, and 0.05 action thresholds in 1995 . The $0.25 \times$ rate of mancozeb was applied three times a week in a total of $22(0), 18$ $(0.0001), 17(0.001), 15(0.01)$, or 14 sprays $(0.05)$. The $1 \times$ fungicidal rate was sprayed weekly for seven $(0)$, six $(0.0001$, and 0.001$)$, or five times $(0.01$ and 0.05$)$. The epidemic rates $\left(r_{G}\right)$ were calculated as [gompit $\left(y_{f}\right)$ - gompit $\left.\left(y_{0}\right)\right] / \Delta t$, where gompit $=-\ln$ $[-\ln (y)], y_{f}=$ final disease proportion, $y_{0}=$ initial disease proportion, and $\Delta t=$ time interval between $t_{0}$ and $t_{f}$. Points on the graph are original data. The lines were generated by nonlinear regression according to the Gompertz model. The oat plants were inoculated on 10 February 1995. 


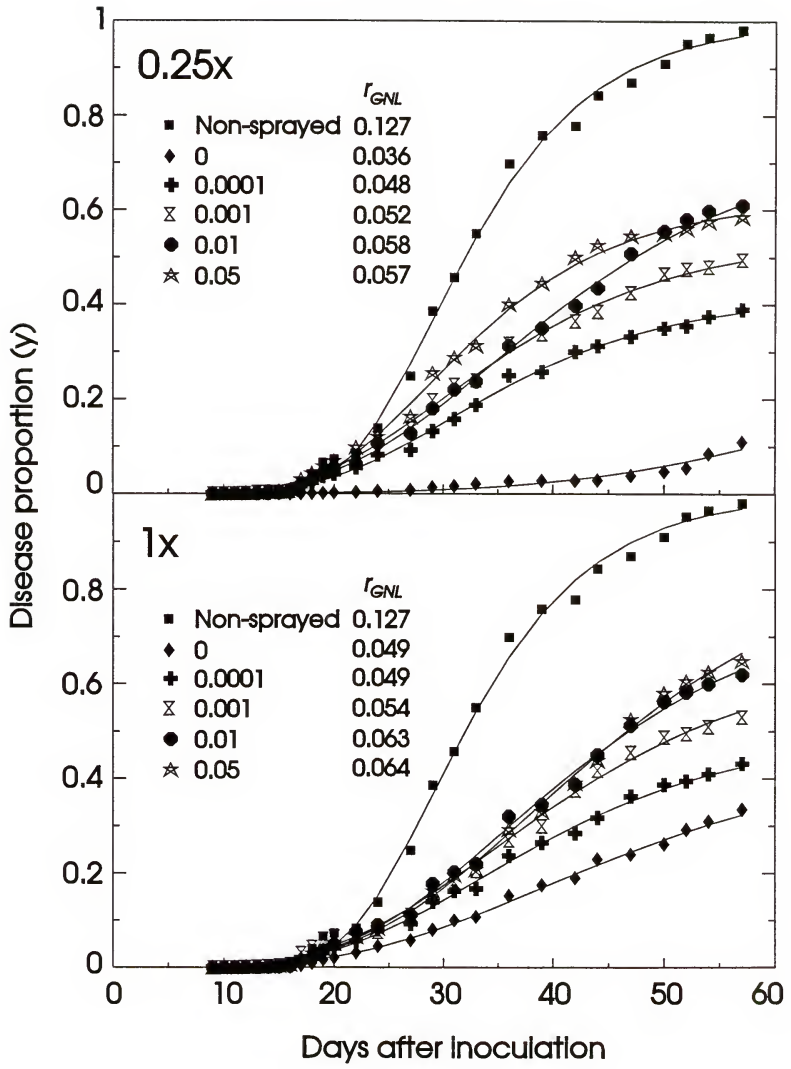




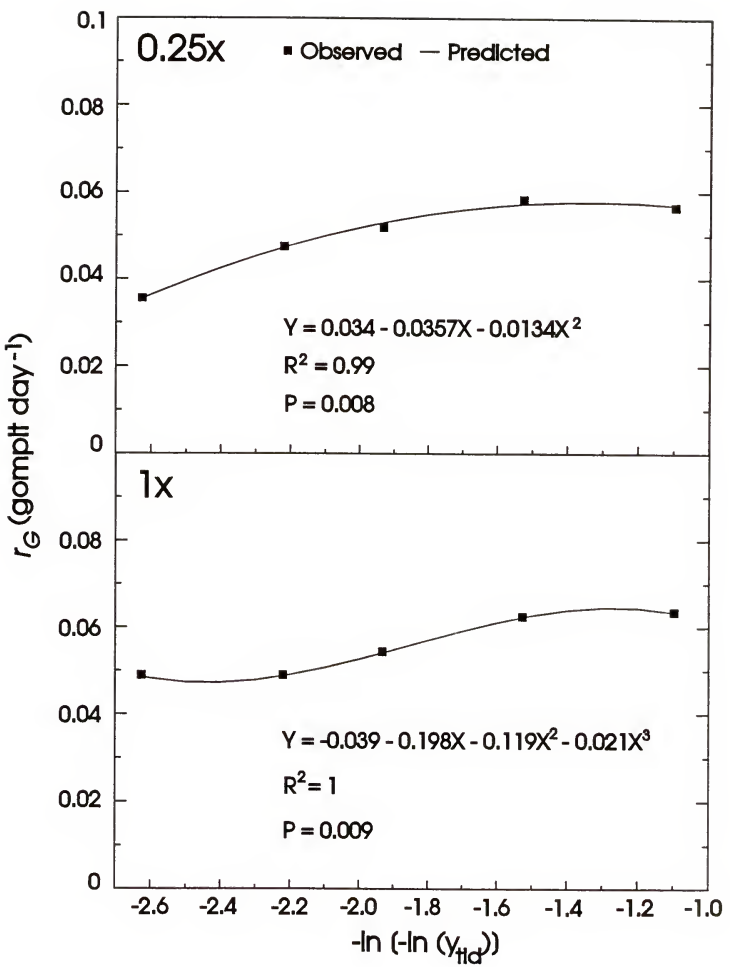

Figure 3.17. Relationship of action thresholds for application of fungicides to epidemic rate $\left(r_{G}\right)$ for crown rust of oats under two fungicidal spray programs in 1995. Applications of mancozeb, at $0.25 \times$ (three times a week) or $1 \times$ (once a week) its label rate, were initiated at $0,0.0001,0.001,0.01$, and 0.05 disease levels. The epidemic rate was plotted against $-\ln \left[-\ln \left(y_{t d d}\right)\right]$, in which $y_{t d d}$ refers to action thresholds. The 0 action threshold was entered as 0.000001 to allow for the transformation above. 
The final amount of disease $\left(y_{f}\right)$ increased as fungicidal sprays began at higher action thresholds (Fig. 3.18). The increase in $y_{f}$ was greater among the $0.25 \times-\left(0.11<y_{f}<\right.$ $0.61)$ than among the $1 x$-spray programs $\left(0.33<y_{f}<0.65\right)$. In both the $0.25 x-$ and $1 x$ spray programs, however, $y_{f}$ increased little or even stabilized for action thresholds between 0.01 and 0.05 . The variation of $y_{f}$ as a function of $y_{t l d}$ was represented by the equations $Y_{0.25 \times}=0.023-0.859 X-0.314 X^{2}, R^{2}=0.99$, and $Y_{1 \times}=0.639-0.111 X-0.088 X^{2}$, $R^{2}=0.98$, where $Y=y_{f}$ and $X=$ gompit $\left(y_{t l d}\right)$.

Table 3.6. Epidemic rates $\left(r_{G}\right)$, final proportions of disease $\left(y_{f}\right)$, areas under the disease progress curves (AUDPC), and oat yields associated with epidemics of crown rust under fungicidal spray programs initiated at various action thresholds in 1995 . The fungicide mancozeb was applied once $(1 \times$ rate $)$ or three times a week $(0.25 \times$ rate). The proportions of disease severity were assessed on flag, $f-1, f-2$, and $f-3$ leaves

\begin{tabular}{|c|c|c|c|c|c|}
\hline \multirow{2}{*}{$\begin{array}{l}\text { Spray program } \\
\text { Action threshold }\end{array}$} & \multicolumn{3}{|c|}{ Epidemic parameter } & \multirow{2}{*}{$\underset{\left(\mathrm{kg} \mathrm{ha}^{-1}\right)}{\text { Yield }}$} & \multirow{2}{*}{$\begin{array}{c}\% \text { Yield } \\
\text { loss }\end{array}$} \\
\hline & $r_{G}^{2}$ & $y_{f}$ & AUDPC & & \\
\hline \multicolumn{6}{|l|}{$\underline{0.25 x}$} \\
\hline 0 & 0.036 & 0.11 & 1.18 & 5975 & - \\
\hline 0.0001 & 0.048 & 0.39 & 8.91 & 5165 & 13.6 \\
\hline 0.001 & 0.052 & 0.49 & 11.63 & 5005 & 16.2 \\
\hline 0.01 & 0.058 & 0.61 & 13.5 & 4745 & 20.6 \\
\hline 0.05 & 0.057 & 0.59 & 14.56 & 4275 & 28.5 \\
\hline Nonsprayed & 0.127 & 0.98 & 23.54 & 2323 & 61.1 \\
\hline \multicolumn{6}{|l|}{$\underline{1 x}$} \\
\hline 0 & 0.049 & 0.33 & 6.28 & 5250 & - \\
\hline 0.0001 & 0.049 & 0.43 & 9.24 & 4990 & 5.0 \\
\hline 0.001 & 0.054 & 0.53 & 11.38 & 4855 & 7.5 \\
\hline 0.01 & 0.063 & 0.62 & 12.74 & 4800 & 8.6 \\
\hline 0.05 & 0.064 & 0.65 & 13.19 & 4745 & 9.6 \\
\hline Nonsprayed & 0.127 & 0.98 & 23.54 & 2323 & 55.8 \\
\hline
\end{tabular}

${ }^{z}$ Epidemic rates were calculated as [gompit $\left(y_{f}\right)$ - gompit $\left.\left(y_{0}\right)\right] / \Delta t$, where gompit $=-\ln [-\ln (y)], y_{f}$ $=$ final disease proportion, $y_{0}=$ initial disease proportion, and $\Delta t=$ time interval between $t_{0}$ and $t_{f}$ 


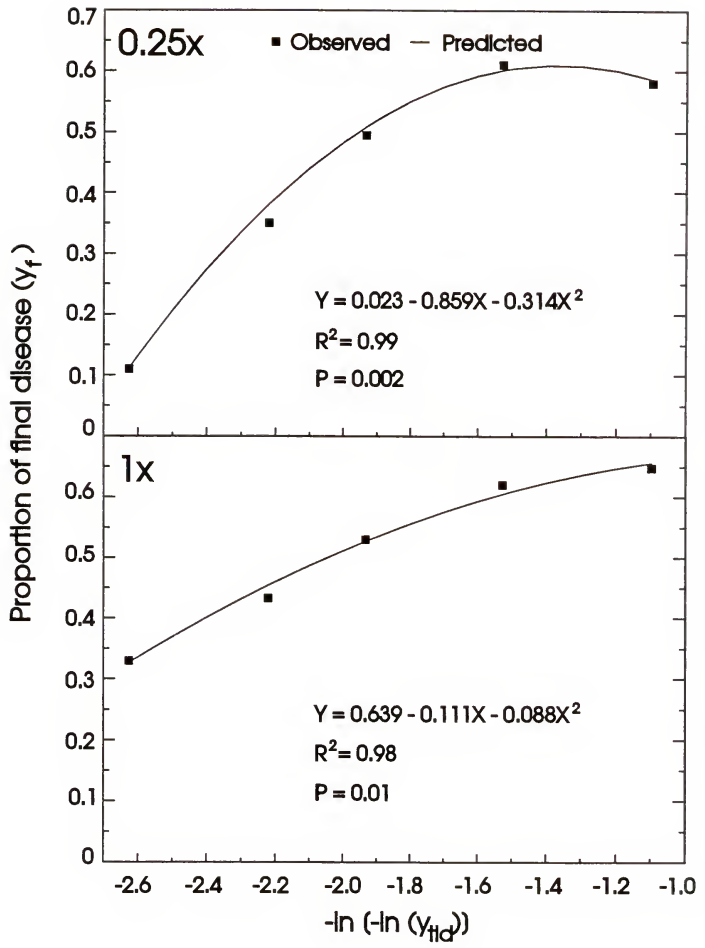

Figure 3.18. Relationship of action thresholds for application of fungicides to proportion of final disease $\left(y_{f}\right)$ for crown rust of oats under two fungicidal spray programs in 1995. Applications of mancozeb, at $0.25 \times$ (three times a week) or $1 \times$ (once a week) its label rate, were initiated at $0,0.0001,0.001,0.01$, and 0.05 disease levels. The proportion of final disease was plotted against $-\ln \left[-\ln \left(y_{t d d}\right)\right]$, in which $y_{t d}$ refers to action thresholds. The 0 action threshold was entered as 0.000001 to allow for the transformation above. 
Application of fungicide according to action thresholds also resulted in higher AUDPCs than those measured in plants sprayed preventively (Fig. 3.19). The equations $Y_{0.25 x}=2.04-19.29 X-7.43 X^{2}, R^{2}=0.99$, and $Y_{1 \times}=9.21-7.09 X-3.14 X^{2}, R^{2}=0.99$, in which $Y$ is AUDPC and $X$ is defined as before, represented the variation in the AUDPC associated with the amount of disease at the time fungicidal sprays were initiated. In both the $0.25 x$ - and $1 \times$ - spray programs, high increase in the AUDPC occurred when fungicidal sprays were postponed until disease severity was 0.0001 . Lower values in AUDPC were observed for action thresholds in the 0.0001-0.05 range.

The less effective control of oat crown rust that resulted from delayed fungicidal sprays affected oat yield negatively. The relationship of kernel yield to the various action thresholds (Fig. 3.20) was as $Y_{0.25 \times}=4233+294.1 X+354.3 X^{2}, R^{2}=0.96$, or $Y_{1 \times}=5147+$ $638.8 X+257.3 X^{2}, R^{2}=0.99$, in which $Y=$ kernel yield and $X=$ gompit of action threshold. The reduction in kernel yield between threshold zero and threshold 0.05 was $505 \mathrm{~kg} \mathrm{ha}^{-1}$ for the $1 \times$ rate and as high as $1700 \mathrm{~kg} \mathrm{ha}^{-1}$ for the $0.25 \times$-spray programs. While little yield reduction was observed for the $1 \times$ rate from threshold 0.01 to threshold 0.05 , a $500 \mathrm{~kg} \mathrm{ha}^{-1}$ yield loss occurred among $0.25 \times$-spray schedules under the same conditions.

In 1996, initiation of fungicidal sprays after the appearance of the first pustules $y_{0}$ $=0.0001)$ resulted in disease severity much higher than that of preventive sprays. The $y_{f}$ and AUDPC were 0.003 and 0.01 for the $0.25 \times$ rate, and 0.08 and 0.58 for the $0.25 \times-$ delay treatment. The delay treatment had an epidemic wave and disease progress curve similar to the standard $1 \times$ treatment (Fig. 3.21). 


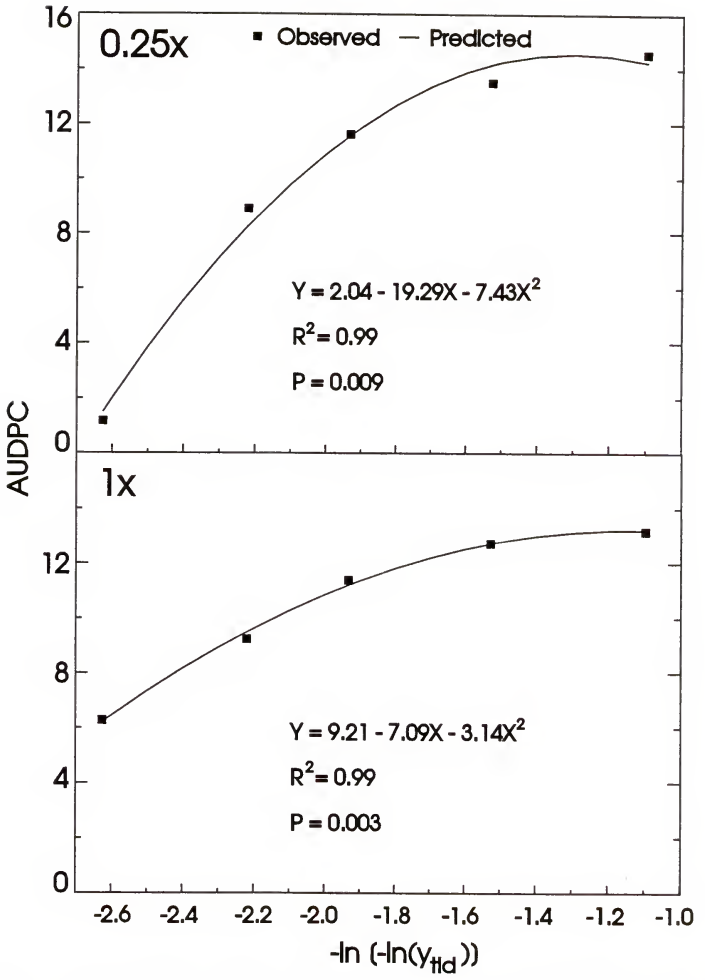

Figure 3.19. Relationship of action thresholds for application of fungicides to area under the disease progress curve (AUDPC) for crown rust of oats under two fungicidal spray programs in 1995. Applications of mancozeb, at $0.25 \times$ (three times a week) or $1 \times$ (once a week) its label rate, were initiated at $0,0.0001,0.001,0.01$, and 0.05 disease levels. The AUDPC was plotted against $-\ln \left[-\ln \left(y_{t d d}\right)\right]$, in which $y_{t d d}$ refers to action thresholds. The 0 action threshold was entered as 0.000001 to allow for the transformation above. 


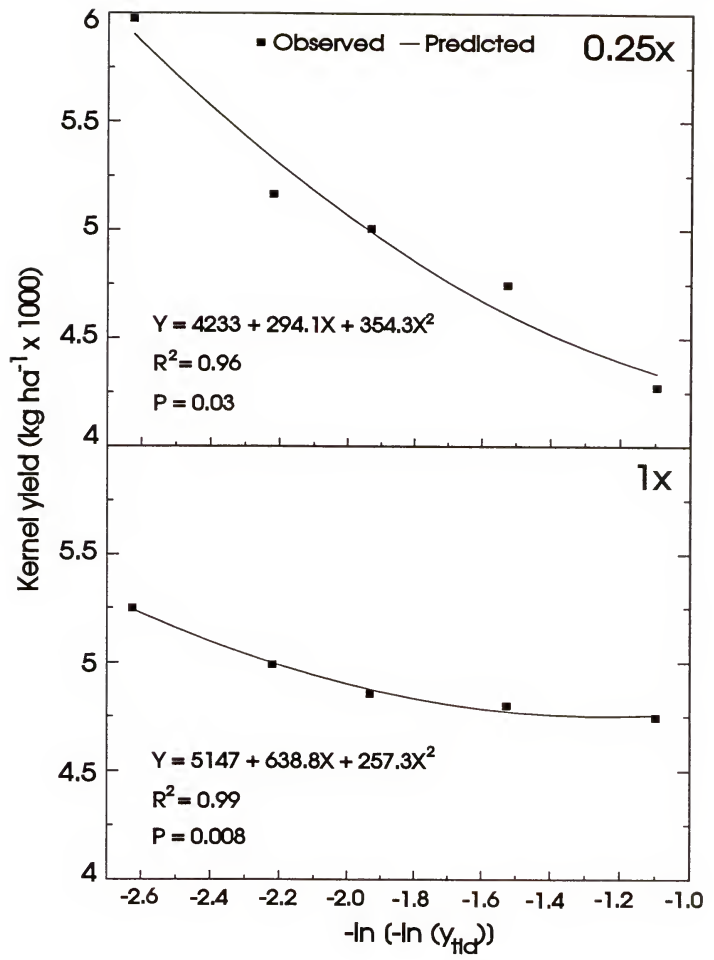

Figure 3.20. Relationship of action thresholds for application of fungicides to kernel yield of oat plants associated with the control of crown rust by two fungicidal spray programs in 1995. Applications of mancozeb, at $0.25 \times$ (three times a week) or $1 \times$ (once a week) its label rate, were initiated at $0,0.0001,0.001,0.01$, and 0.05 disease levels. Kernel yield was plotted against $-\ln \left[-\ln \left(y_{t t d}\right)\right]$, in which $y_{t d d}$ refers to action thresholds. The 0 action threshold was entered as 0.000001 to allow for the transformation above. 
Figure 3.21. Disease progress curves (A) and epidemic waves (B) for crown rust of oats under fungicidal spray programs initiated at different levels of disease severity in 1996. The $0.25 \times$ rate of mancozeb was applied at inoculation (day 100) and repeated twice a week for seven times. The delay treatment $(0.25 x$-delay) was applied three times from the 0.0001 disease level. The standard $1 \times$ treatment was applied twice on days 109 and 120. The epidemic rates $\left(r_{G N L}\right)$ were estimated by nonlinear regression of disease proportions against time using the Gompertz model. The disease proportions $(y)$ were transformed by $-\ln [-\ln (y)]$ to show the epidemic waves, whose ends are indicated by arrows. This experiment was planted on 2 December 1995. 


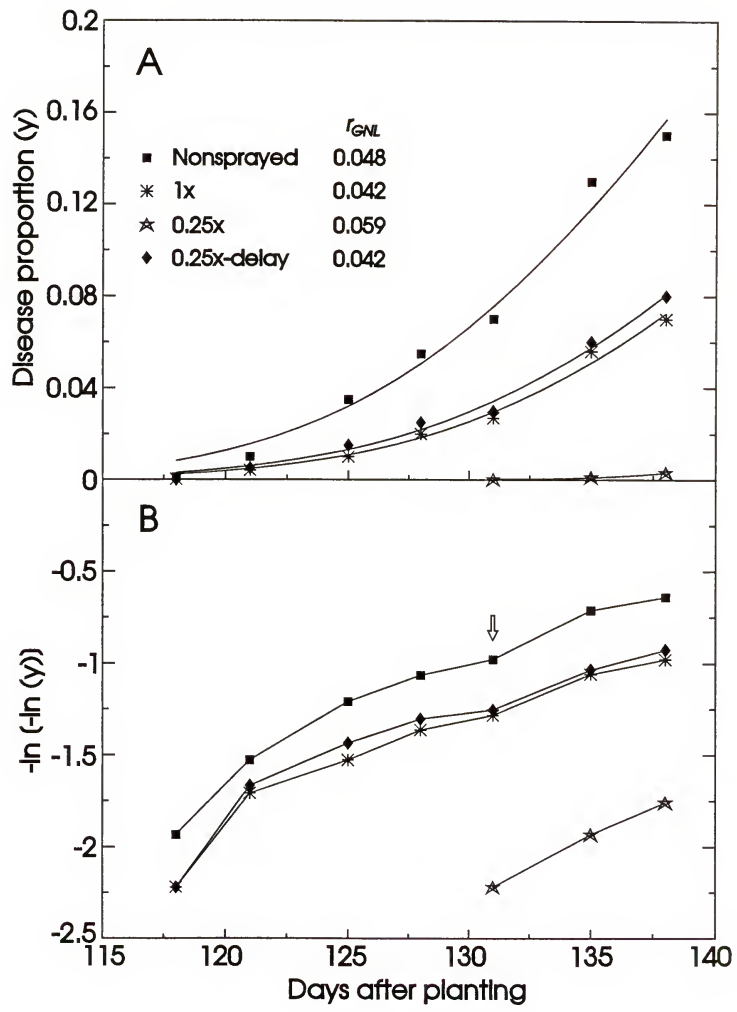


Significant variation in disease intensity associated with action thresholds was also observed in the experiment conducted in 1997 (Table 3.7 and Fig. 3.22). In this experiment, the $0.0001,0.001$, and 0.01 action thresholds were detected at 10,14 , and 16 days after inoculation, that is, all thresholds occurred during the first epidemic wave. Although fungicidal treatments were initiated the same day their respective action thresholds were detected, the presence of latent infections precluded fungicidal sprays of having major impact on the epidemic waves (Fig. 3.23). These waves were very similar to that of nonsprayed plants.

Table 3.7. Estimated epidemic rates $\left(r_{G N L}\right)$, final proportions of disease $\left(y_{f}\right)$, areas under the disease progress curves (AUDPC), and oat yields associated with epidemics of crown rust under fungicidal spray programs initiated at various action thresholds in 1997. The fungicide mancozeb was applied at weekly $(1 \times)$ or twice-weekly intervals $(0.25 \times)$. The proportions of disease severity were assessed on flag, $f-1, f-2$, and $f-3$ leaves

\begin{tabular}{|c|c|c|c|c|c|c|}
\hline \multirow{2}{*}{$\frac{\text { Spray program }}{\text { Action threshold }}$} & \multicolumn{3}{|c|}{ Epidemic parameter } & \multirow{2}{*}{$\begin{array}{c}\text { Yield } \\
\left(\mathrm{kg} \mathrm{ha}^{-1}\right)\end{array}$} & \multirow{2}{*}{$\begin{array}{c}\% \\
\text { Yield } \\
\text { loss } \\
\end{array}$} & \multirow{2}{*}{$\begin{array}{c}\text { Thousand } \\
\text {-kernel } \\
\text { weight (g) }\end{array}$} \\
\hline & $r_{G N L}{ }^{2}$ & $y_{f}$ & AUDPC & & & \\
\hline \multicolumn{7}{|l|}{$\underline{0.25 x}$} \\
\hline 0 & 0.042 & 0.21 & 0.93 & 4139 & - & 28.6 \\
\hline 0.0001 & 0.05 & 0.5 & 7.49 & 3057 & 26.1 & 26.8 \\
\hline 0.001 & 0.062 & 0.62 & 9.61 & 2651 & 36.8 & 24.1 \\
\hline 0.01 & 0.069 & 0.72 & 11.36 & 2367 & 42.8 & 22.7 \\
\hline Nonsprayed & 0.134 & 0.96 & 17.2 & 1532 & 63.0 & 20.8 \\
\hline \multicolumn{7}{|l|}{$\underline{1 x}$} \\
\hline 0 & 0.048 & 0.33 & 3.4 & 3961 & - & 28.8 \\
\hline 0.0001 & 0.049 & 0.47 & 7.12 & 3396 & 14.3 & 26.3 \\
\hline 0.001 & 0.058 & 0.59 & 9.07 & 2860 & 27.8 & 25.5 \\
\hline 0.01 & 0.074 & 0.78 & 11.51 & 2700 & 31.8 & 24.3 \\
\hline Nonsprayed & 0.134 & 0.96 & 17.2 & 1532 & 61.3 & 20.8 \\
\hline
\end{tabular}

${ }^{2}$ Epidemic rates were estimated by nonlinear regression of disease proportions against time using the Gompertz model.

The estimated epidemic rate $\left(r_{G N L}\right)$, the final amount of disease $\left(y_{f}\right)$, and the AUDPC increased proportionally to the amount of disease at the time each fungicidal 
Figure 3.22. Disease progress curves for crown rust epidemics subjected to fungicidal spray programs initiated at the $0,0.0001,0.001$, and 0.01 action thresholds in 1997 . The $0.25 \times$ rate of mancozeb was applied twice a week in a total of $12(0)$, nine $(0.0001)$, or eight sprays ( 0.001 and 0.01$)$. The $1 \times$ fungicidal rate was sprayed weekly for six $(0)$, five $(0.0001)$, or four times $(0.001$ and 0.01$)$. The epidemic rates $\left(r_{G N L}\right)$ were estimated by nonlinear regression of disease proportions against time using the Gompertz model. The oat plants were inoculated on 15 February 1997. 


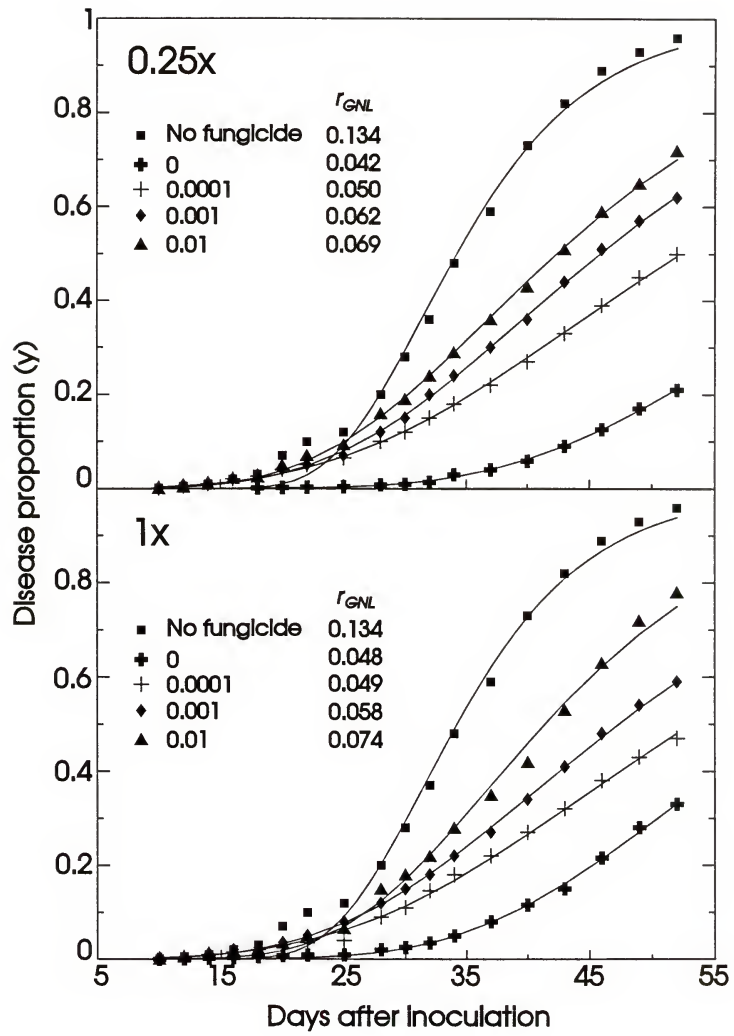


Figure 3.23. Epidemic waves for crown rust epidemics subjected to fungicidal spray programs initiated at the $0,0.0001,0.001$, and 0.01 action thresholds in 1997 . The $0.25 \times$ rate of mancozeb was applied twice a week in a total of $12(0)$, nine $(0.0001)$, or eight sprays $(0.001$ and 0.01$)$. The $1 \times$ fungicidal rate was sprayed weekly for six $(0)$, five $(0.0001)$, or four times $(0.001$ and 0.01$)$. The disease proportions $(y)$ were transformed by $-\ln [-\ln (y)]$ to show the epidemic waves, whose ends are indicated by arrows. The oat plants were inoculated on 15 February 1997. 


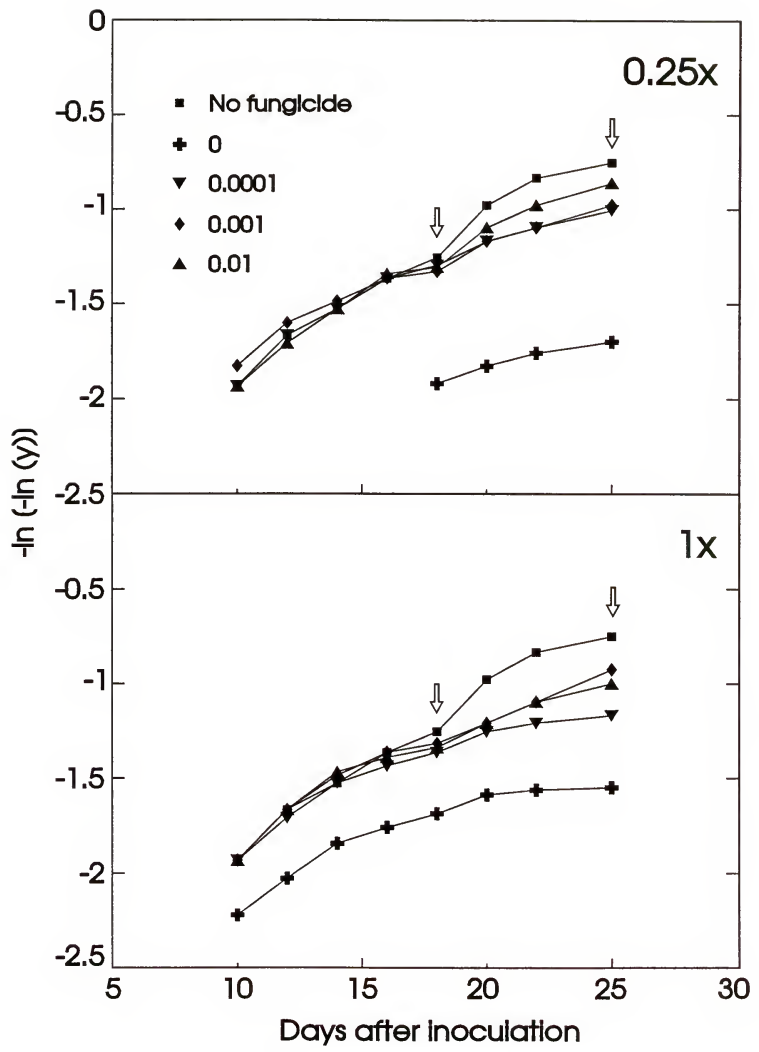


spray program was initiated. This effect was always greater among the $0.25 \times$ - than among the $1 \times$-spray schedules (Table 3.7). The relationships of action thresholds, expressed as $-\ln \left[-\ln \left(y_{t d}\right)\right]$, to the epidemic parameters $\left(r_{G N L}, y_{\beta}\right.$ and AUDPC) were represented by quadratic equations (Figs. 3.24-3.26), in which crown rust increased as spray applications were delayed. The effect of action thresholds on the epidemic rate among the low-rate treatments was a straight line (Fig. 3.24). The yield and quality of oat kernels significantly $(P=0.03)$ decreased as fungicidal treatments were initiated at higher levels of disease severity (Fig. 3.27 and 3.28).

\section{Duration of Fungicidal Schedules on the Management of Crown Rust}

Various spray programs initiated at inoculation were discontinued $21\left(T_{21}\right), 28$ $\left(T_{28}\right), 35\left(T_{35}\right), 42\left(T_{42}\right)$, and 49 days $\left(T_{49}\right)$ later. The disease severities at the time each spray program was ceased were $0.023\left(T_{21}\right), 0.043\left(T_{28}\right), 0.064\left(T_{35}\right), 0.076\left(T_{42}\right)$, and 0.09 $\left(T_{49}\right)$ for the $0.25 \times$-rate treatments, and $0.042\left(T_{21}\right), 0.071\left(T_{28}\right), 0.126\left(T_{35}\right), 0.185\left(T_{42}\right)$, and $0.235\left(T_{49}\right)$ for $1 \times$-rate treatments. The epidemic parameters and disease progress curves for the various spray programs tested in this experiment are shown in Table 3.8 and Figure 3.29.

Cessation of fungicidal sprays shortly after inoculation resulted in high epidemic rates. For instance, $r_{G}$ was as high as $0.064(0.25 \times)$ and 0.069 gompits day $^{-1}(1 \times)$ in spray programs discontinued at 21 days after inoculation. For both the $0.25 \times$ - and the $1 \times$-spray programs, $r_{G}$ decreased as fungicidal sprays were extended to 28,35 , or 42 days (Fig. 3.30). Fungicidal sprays discontinued at 42 or 49 days after inoculation had similar $r_{G} \mathrm{~s}$. The relationship of $r_{G}$ to duration of spray program was represented by the equations $Y_{0.25 \times}$ 


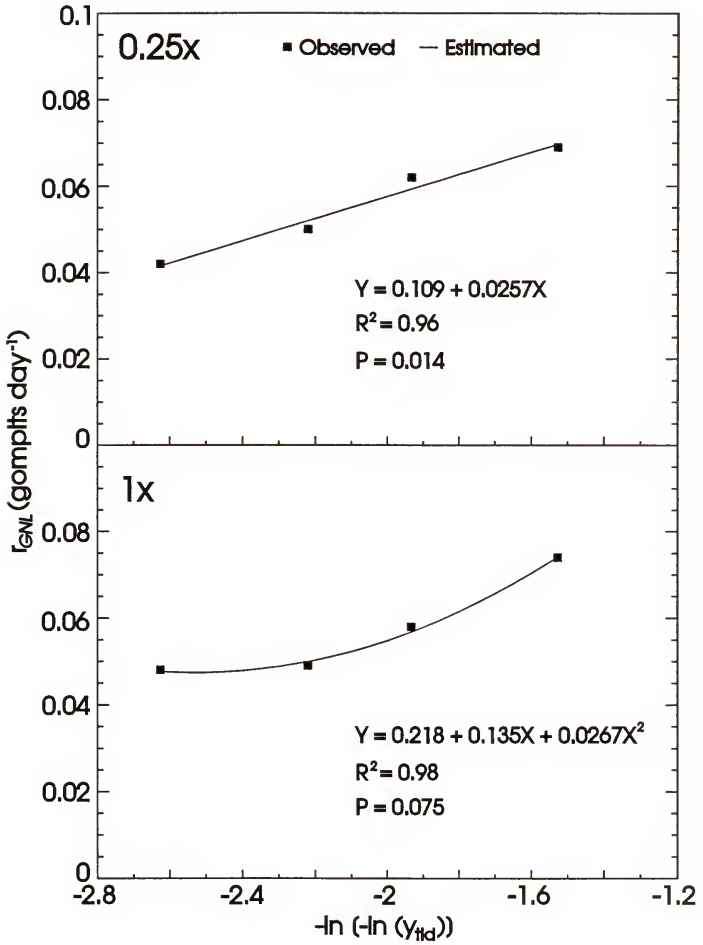

Figure 3.24. Relationship of action thresholds for application of fungicides to estimated epidemic rate $\left(r_{G N L}\right)$ for crown rust of oats under two fungicidal spray programs in 1997. Applications of mancozeb, at $0.25 \times$ (twice a week) or $1 \times$ (once a week) its label rate, were initiated at $0,0.0001,0.001$, and 0.01 disease levels. The epidemic rate was plotted against $-\ln \left[-\ln \left(y_{t d d}\right)\right]$, in which $y_{\text {tld }}$ refers to action thresholds. The 0 action threshold was entered as 0.000001 to allow for the transformation above. 


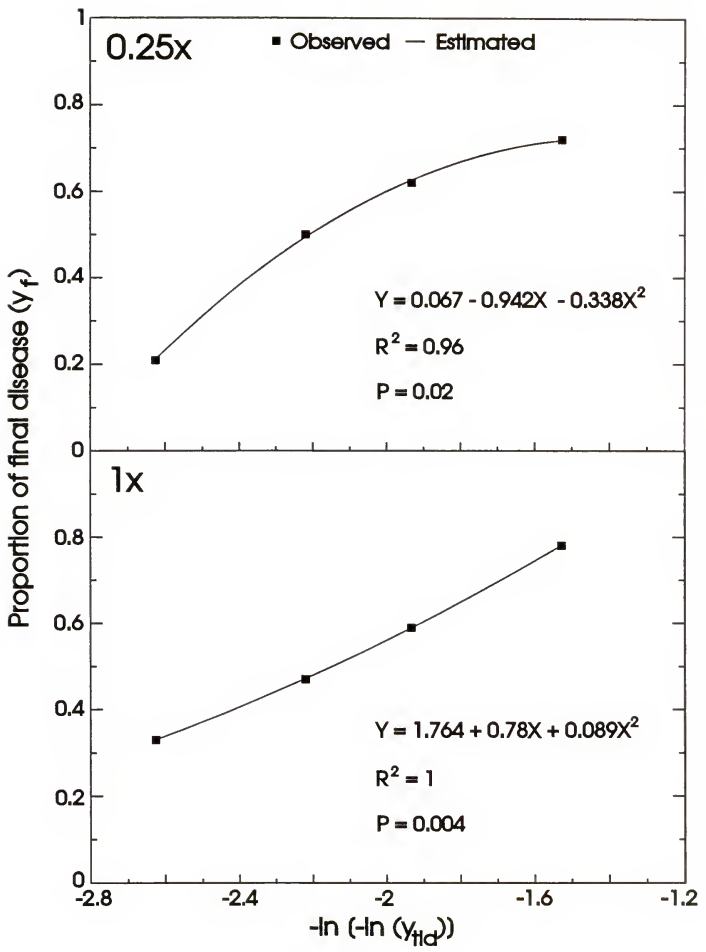

Figure 3.25. Relationship of action thresholds for application of fungicides to proportion of final disease $\left(y_{f}\right)$ for crown rust of oats under two fungicidal spray programs in 1997. Applications of mancozeb, at $0.25 \times$ (twice a week) or $1 \times$ (once a week) its label rate, were initiated at $0,0.0001,0.001$, and 0.01 disease levels. The proportion of final disease was plotted against $-\ln \left[-\ln \left(y_{t d d}\right)\right]$, in which $y_{t l d}$ refers to action thresholds. The 0 action threshold was entered as 0.000001 to allow for the transformation above. 


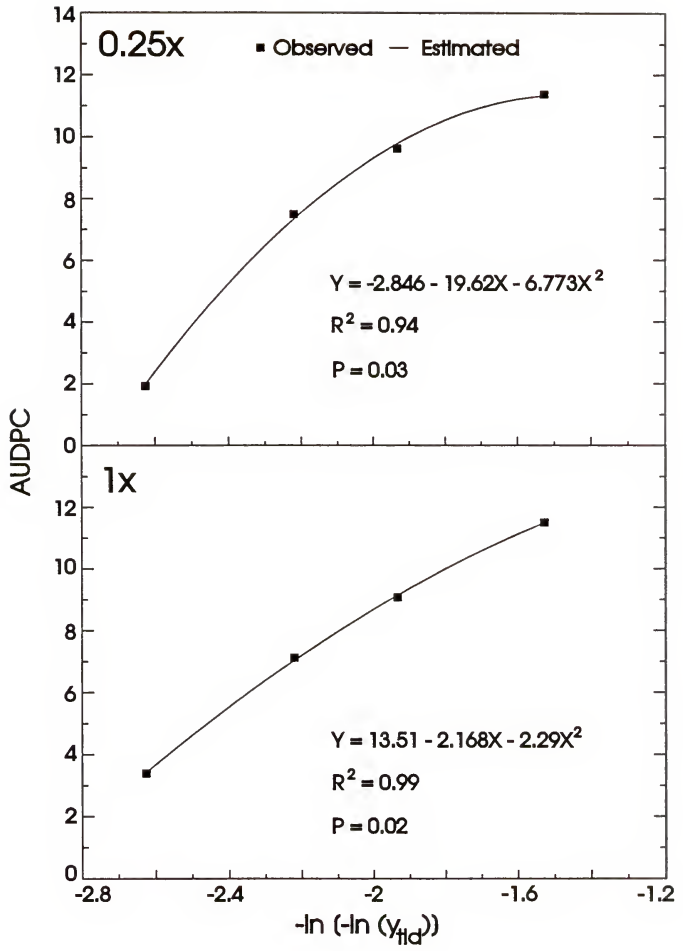

Figure 3.26. Relationship of action thresholds for application of fungicides to area under the disease progress curve (AUDPC) for crown rust of oats under two fungicidal spray programs in 1997. Applications of mancozeb, at $0.25 \times$ (twice a week) or $1 \times$ (once a week) its label rate, were initiated at $0,0.0001,0.001$, and 0.01 disease levels. The AUDPC was plotted against $-\ln \left[-\ln \left(y_{t t}\right)\right]$, in which $y_{t d}$ refers to action thresholds. The 0 action threshold was entered as 0.000001 to allow for the transformation above. 


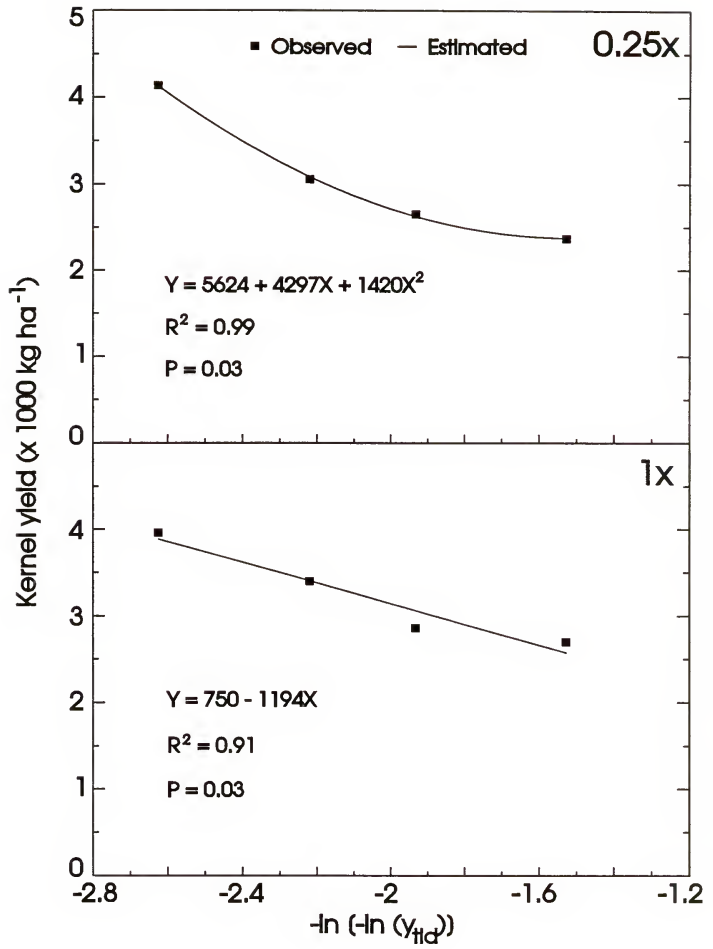

Figure 3.27. Relationship of action thresholds for application of fungicides to kernel yield of oat plants associated with the control of crown rust by two fungicidal spray programs in 1997. Applications of mancozeb, at $0.25 \times$ (twice a week) or $1 \times$ (once a week) its label rate, were initiated at $0,0.0001,0.001$, and 0.01 disease levels. Kernel yield was plotted against $-\ln \left[-\ln \left(y_{t d d}\right)\right]$, in which $y_{t d}$ refers to action thresholds. The 0 action threshold was entered as 0.000001 to allow for the transformation above. 


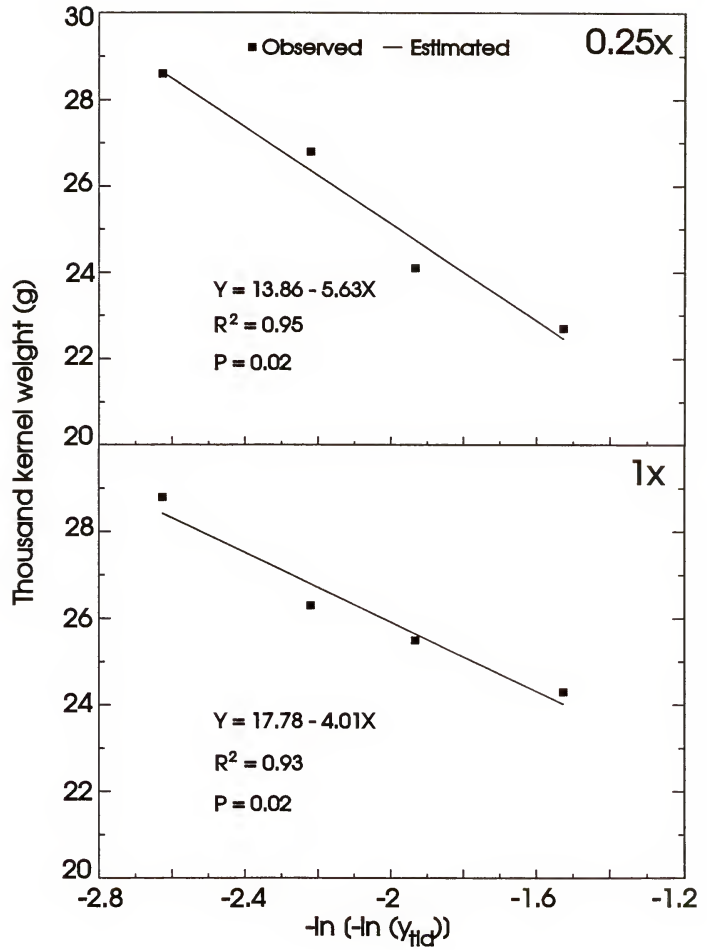

Figure 3.28. Relationship of action thresholds to thousand kernel weight (TKW) of oat plants associated with the control of crown rust by two fungicidal spray programs in 1997. The fungicide mancozeb was applied at $0.25 \times$ (twice a week) or at $1 \times$ (once a week) its label rate. The TKW was plotted against $-\ln \left[-\ln \left(y_{t l d}\right)\right]$, in which $y_{t l d}$ refers to the $0,0.0001,0.001$, and 0.01 action thresholds. The 0 action threshold was entered as 0.000001 to allow for the transformation above. 
Figure 3.29. Disease progress curves for epidemics of crown rust of oats under fungicidal spray programs discontinued at $21,28,35,42$, and 49 days after inoculation in 1995 . The fungicide mancozeb was applied at $0.25 \times$ (three times a week) or $1 \times$ (once a week) its label rate. The epidemic rates $\left(r_{G}\right)$ were calculated as [gompit $\left(y_{f}\right)$ - gompit $\left.\left(y_{0}\right)\right] / \Delta t$, where gompit $=-\ln [-\ln (y)], y_{f}=$ final disease proportion, $y_{0}=$ initial disease proportion, and $\Delta t=$ time interval between $t_{0}$ and $t_{f}$. Points on the graph are original data. The lines were generated by nonlinear regression according to the Gompertz model. This experiment was planted on 3 December 1994 and inoculated on 10 February 1995. 


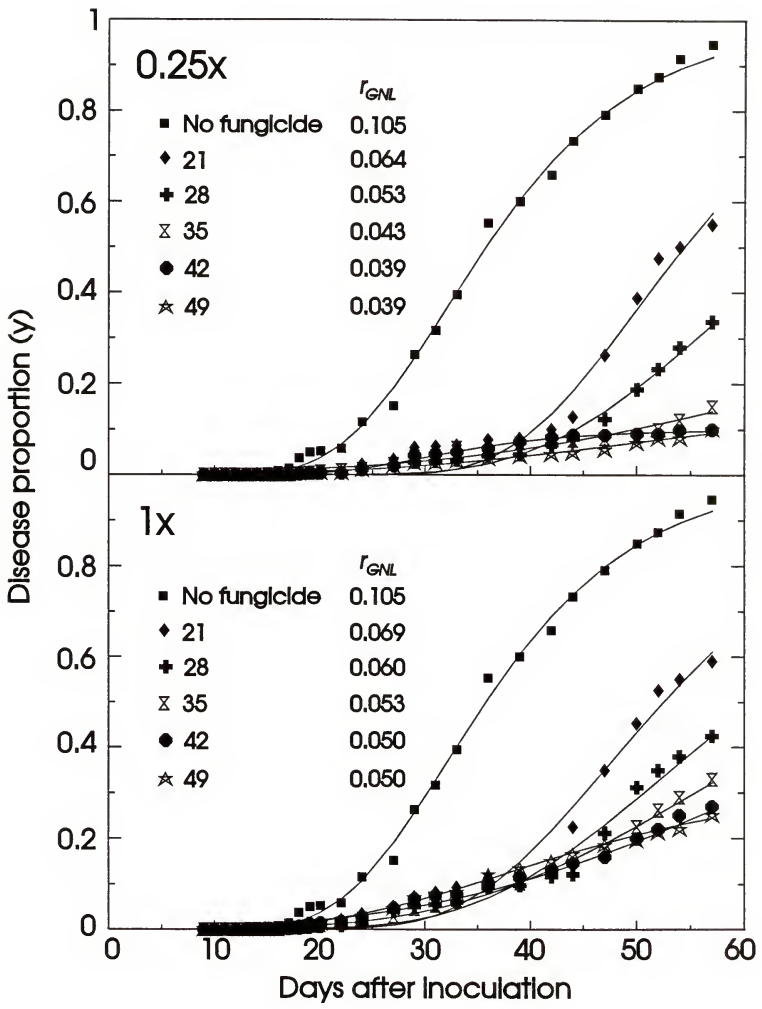


$=0.1256-0.0038 X+0.00004 X^{2}, R^{2}=0.99$, and $Y_{1 \times}=0.1165-0.0029 X+0.00003 X^{2}, R^{2}=$

1 , in which $Y$ is $r_{G}$ and $X$ is duration of spray program in days.

The final amount of crown rust was also affected by duration of spray programs.

The $y_{f}$ was highest on treatments discontinued 21 days after inoculation $\left(T_{21}\right)$. As fungicidal sprays were extended to $28,35,42$, and 49 days, $y_{f}$ decreased curvilinearly

(Fig. 3.31). The equations $Y_{0.25 \times}=1.74-0.074 X+0.0008 X^{2}, R^{2}=0.99$, and $Y_{1 \times}=1.324-$ $0.045 X+0.00047 X^{2}, R^{2}=0.99$, represented the effects of duration of fungicidal sprays $(X)$ on $y_{f}(Y)$. In both $1 \times-$ and $0.25 \times$ - spray programs, $y_{f}$ s between spray schedules discontinued at 42 and 49 days after inoculation were very similar to each other.

Table 3.8. Epidemic rates $\left(r_{G}\right)$, final proportions of disease $\left(y_{f}\right)$, areas under the disease progress curves (AUDPC), and oat yields associated with epidemics of crown rust subjected to fungicidal spray programs discontinued at various periods after inoculation. The fungicide mancozeb was applied once a week $(1 \times)$ or three times a week $(0.25 \times)$. The proportions of disease severity were assessed on flag, $f-1, f-2$, and $f-3$ leaves. Experiment developed in 1995

\begin{tabular}{|c|c|c|c|c|c|}
\hline \multirow{2}{*}{$\frac{\text { Spray program }}{\text { Duration (days) }}$} & \multicolumn{3}{|c|}{ Epidemic parameter } & \multirow{2}{*}{$\begin{array}{c}\text { Yield } \\
\left(\mathrm{kg} \mathrm{ha}^{-1}\right)\end{array}$} & \multirow{2}{*}{$\begin{array}{c}\% \text { Yield } \\
\text { loss }\end{array}$} \\
\hline & $r_{G}^{2}$ & $\boldsymbol{y}_{f}$ & AUDPC & & \\
\hline \multicolumn{6}{|l|}{$\underline{0.25 x}$} \\
\hline 21 & 0.086 & 0.55 & 6.39 & 5025 & 9.5 \\
\hline 28 & 0.058 & 0.34 & 3.43 & 5410 & 2.5 \\
\hline 35 & 0.021 & 0.15 & 2.39 & 5520 & 0.5 \\
\hline 42 & 0.123 & 0.1 & 1.88 & 5565 & +0.3 \\
\hline 49 & 0.038 & 0.1 & 1.57 & 5550 & - \\
\hline Nonsprayed & 0.099 & 0.95 & 20.03 & 3160 & 43.1 \\
\hline \multicolumn{6}{|l|}{$\underline{1 \times}$} \\
\hline 21 & 0.075 & 0.59 & 7.89 & 4890 & 9.1 \\
\hline 28 & 0.054 & 0.43 & 5.40 & 5070 & 5.8 \\
\hline 35 & 0.039 & 0.33 & 4.62 & 5210 & 3.2 \\
\hline 42 & 0.029 & 0.27 & 4.53 & 5315 & 1.2 \\
\hline 49 & 0.062 & 0.25 & 4.41 & 5380 & - \\
\hline Nonsprayed & 0.099 & 0.95 & 20.03 & 3160 & 41.3 \\
\hline
\end{tabular}




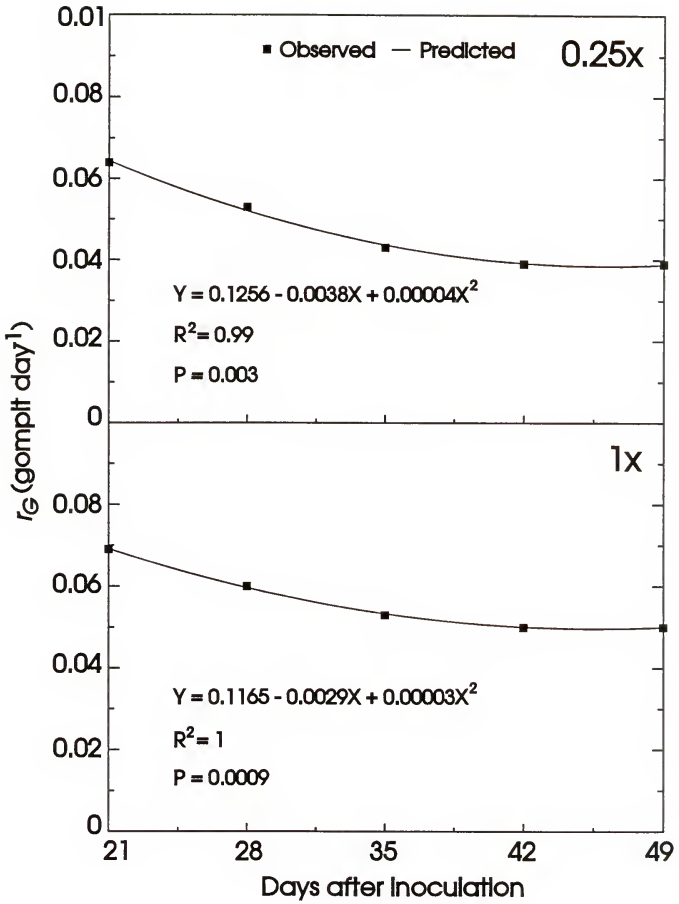

Figure 3.30. Relationship of duration of fungicidal treatments to epidemic rate $\left(r_{G}\right)$ for crown rust of oats under two spray programs in 1995. Applications of the fungicide mancozeb, at $0.25 \times$ (three times a week) or $1 \times$ (once a week), were initiated at inoculation and discontinued $21,28,35,42$, and 49 days later. The epidemic rates $\left(r_{G}\right)$ were calculated as [gompit $\left(y_{f}\right)$ - gompit $\left.\left(y_{0}\right)\right] / \Delta t$, where gompit $=-\ln [-\ln (y)], y_{f}=$ final disease proportion, $y_{0}=$ initial disease proportion, and $\Delta t=$ time interval between $t_{0}$ and $t_{f}$ This experiment was planted on 3 December 1994 and inoculated on 10 February 1995. 


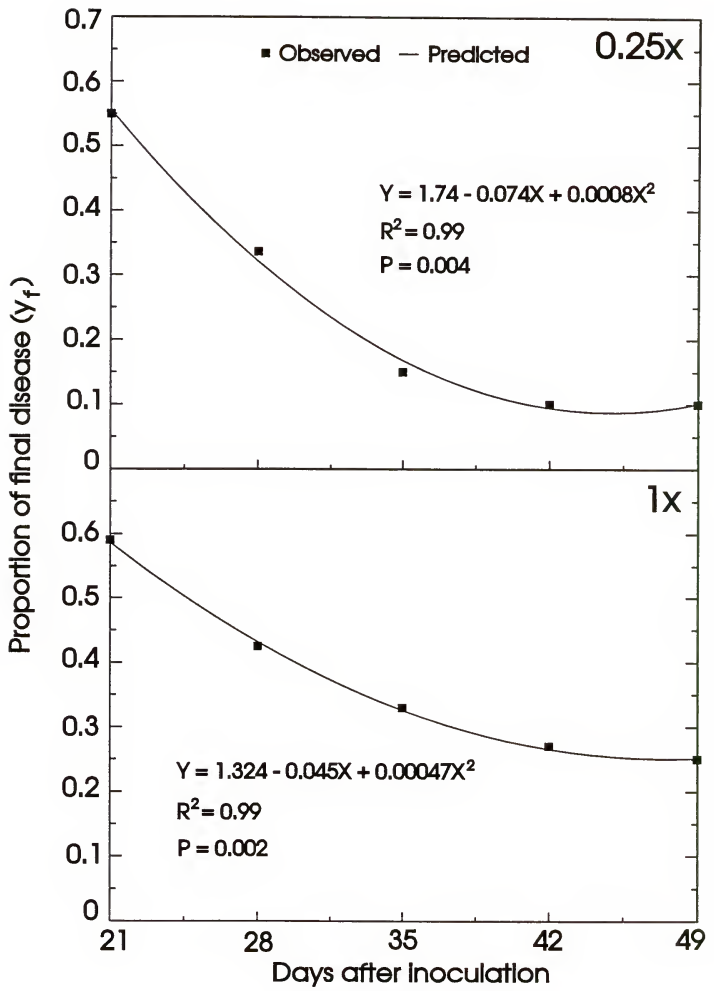

Figure 3.31. Relationship of duration of fungicidal treatments to proportion of final disease $\left(y_{f}\right)$ for crown rust of oats under two spray programs in 1995. Applications of the fungicide mancozeb, at $0.25 \times$ (three times a week) or $1 \times$ (once a week), were initiated at inoculation and discontinued $21,28,35,42$, and 49 days later. This experiment was planted on 3 December 1994 and inoculated on 10 February 1995. 
Fungicidal schedules discontinued earlier accumulated more disease along the epidemic than long-term spray programs. For example, the AUDPCs for $T_{21}$ were as high as $6.39(0.25 \times)$ and $7.89(1 \times)$. Although the lowest AUDPCs were observed for $T_{49}$, most of the reduction in AUDPC occurred between $T_{21}$ and $T_{35}$ (Fig. 3.32). The variation in AUDPC associated with duration of fungicidal treatments was higher in $0.25 x-$ than in 1x-fungicidal schedules. The equations $Y_{0.25 x}=18.3-0.758 X+0.0085 X^{2}, R^{2}=0.98$, and $Y_{1 \mathrm{x}}=18.2-0.666 X+0.0079 X^{2}, R^{2}=0.96$, represented the effect of duration of fungicidal treatments on AUDPC.

The oat kernel yield was correlated with duration of fungicidal treatments, that is, higher yields were obtained from plants subjected to longer spray programs (Fig. 3.33). The difference in yield between $T_{21}$ and $T_{49}$ was $490 \mathrm{~kg} \mathrm{ha}^{-1}$ for the $1 \times$ rate and $525 \mathrm{~kg}$ $\mathrm{ha}^{-1}$ for the $0.25 \times$ rate. The oat yield tended to stabilize after $T_{35}$, especially for spray programs based on the reduced $(0.25 \times)$ fungicidal rate. The equations $Y_{0.25 \times}=3390+$ $105.5 X-1.26 X^{2}, R^{2}=0.96$, and $Y_{1 \times}=4125+44.54 X-0.386 X^{2}, R^{2}=1$, fit the relationship of yield $(Y)$ to duration of fungicidal programs $(X)$.

\section{Single- and Mixed-Rate Fungicidal Schedules for Management of Oat Crown Rust}

The effect of mixed fungicidal schedules on epidemics of crown rust was tested in experiments developed in 1996 and 1997. In 1996, the short epidemics and the low levels of disease severity did not provide enough information to compare the effects of such treatments on disease control and yield. Therefore, data on mixed schedules from the 1996 experiment were not analyzed. In 1997, two multiple-rate treatments, based on early season sprays of $0.25 \times$ mancozeb followed by sprays of the same fungicide at its 


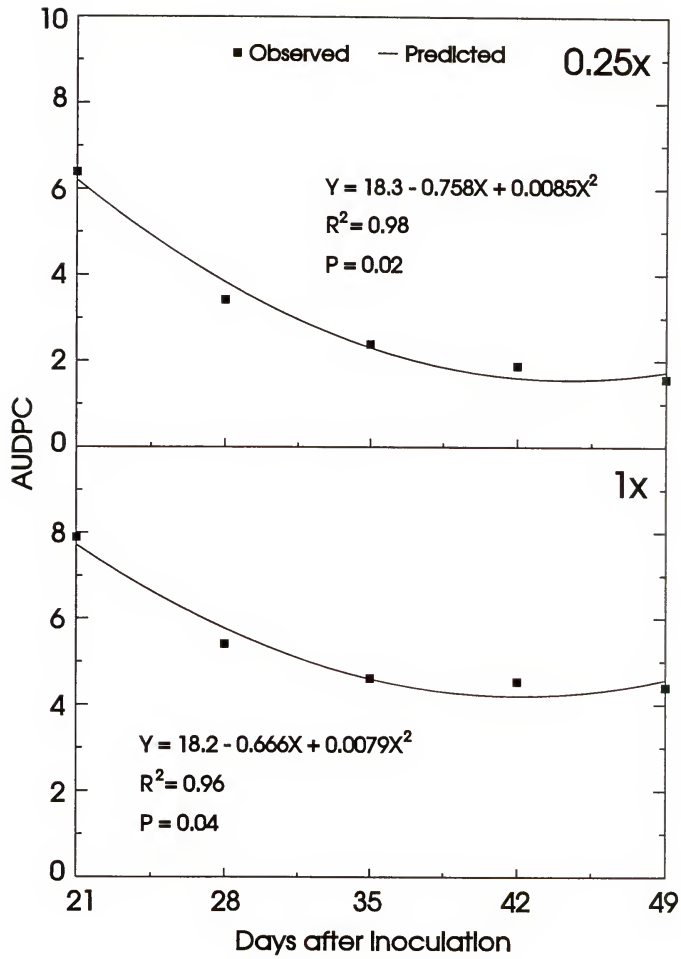

Figure 3.32. Relationship of duration of fungicidal treatments to area under the disease progress curve (AUDPC) for crown rust of oats under two spray programs in 1995. Applications of the fungicide mancozeb, at $0.25 \times$ (three times a week) or $1 \times$ (once a week), were initiated at inoculation and discontinued 21, 28, 35, 42, and 49 days later. This experiment was planted on 3 December 1994 and inoculated on 10 February 1995. 


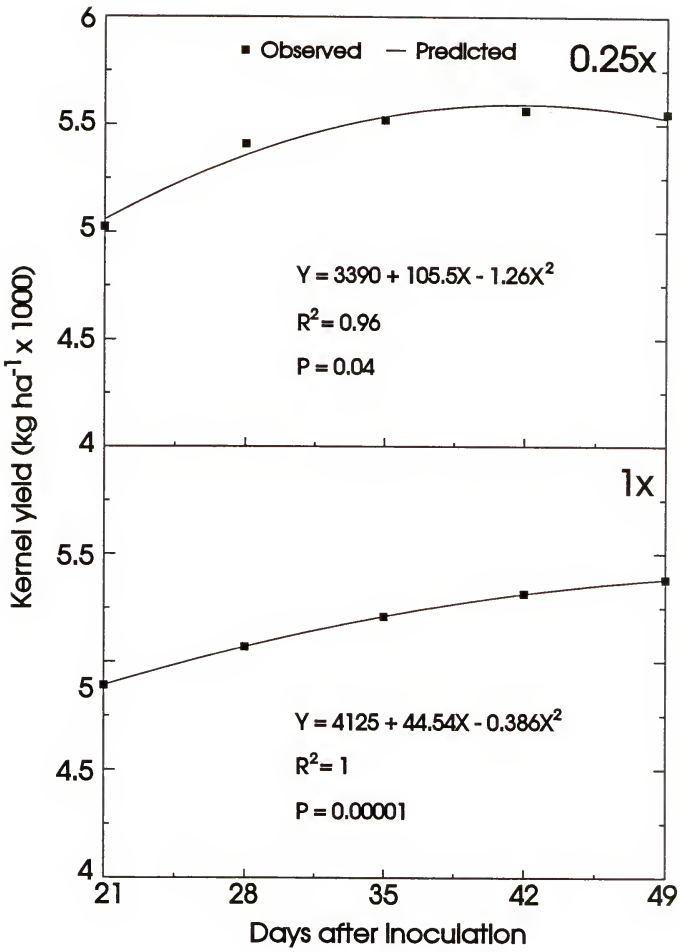

Figure 3.33. Relationship of duration of fungicidal treatments to kernel yield of oat plants subjected to two spray programs to control crown rust in 1995. Applications of the fungicide mancozeb, at $0.25 \times$ (three times a week) or $1 \times$ (once a week), were initiated at inoculation and discontinued $21,28,35,42$, and 49 days later. This experiment was planted on 3 December 1994 and inoculated on 10 February 1995. 
full rate, were compared to single-rate spray schedules of $0.25 \times$ or $1 \times$ mancozeb. The mixed schedule I comprised four sprays of the $0.25 \times$ rate plus two sprays of the $1 \times$ rate. The mixed schedule II had six sprays of $0.25 \times$ mancozeb and two sprays of $1 \times$ mancozeb. The single-rate spray programs involved 12 sprays of the $0.25 \times$ rate or three sprays of the full rate. The spray intervals were always 3-4 days for the lower rate and 10-12 days for the full rate.

Table 3.9. Estimated epidemic rates $\left(r_{G N L}\right)$, final proportions of disease $\left(y_{f}\right)$, and areas under the disease progress curve (AUDPC) associated with epidemics of crown rust under single- and multiple-rate spray schedules of the fungicide mancozeb in 1997. The proportions of disease severity were assessed on flag, $f-1, f-2$, and $f-3$ leaves

\begin{tabular}{lcccc}
\hline \multirow{2}{*}{$\begin{array}{l}\text { Fungicidal } \\
\text { spray program }\end{array}$} & $\begin{array}{c}\text { Number of } \\
\text { sprays }\end{array}$ & \multicolumn{4}{c}{ Epidemic parameter } \\
\cline { 3 - 5 } & & $\boldsymbol{r}_{G N L}{ }^{\mathrm{y}}$ & $\boldsymbol{y}_{f}$ & AUDPC \\
\hline $0.25 \times$ & 12 & $0.042 \mathrm{a}^{\mathrm{z}}$ & $0.21 \mathrm{a}$ & $1.93 \mathrm{a}$ \\
Mixed II & $6+2$ & $0.044 \mathrm{ab}$ & $0.25 \mathrm{a}$ & $2.15 \mathrm{a}$ \\
Mixed I & $4+2$ & $0.048 \mathrm{bc}$ & $0.29 \mathrm{a}$ & $2.41 \mathrm{a}$ \\
Standard 1x & 3 & $0.052 \mathrm{c}$ & $0.47 \mathrm{~b}$ & $6.6 \mathrm{~b}$ \\
Nonsprayed & - & $0.134 \quad \mathrm{~d}$ & $0.96 \mathrm{c}$ & $17.2 \quad \mathrm{c}$ \\
\hline P $>$ F & & 0.001 & 0.0005 & 0.0001 \\
\hline
\end{tabular}

${ }^{y}$ Epidemic rates were estimated by nonlinear regression of disease proportions against time using the Gompertz model.

${ }^{z}$ Treatment means within columns followed by the same letter do not differ significantly $(P<$ 0.05 ) by Duncan's Multiple Range Test, using the variability pooled among replicates.

All three fungicidal programs that included applications of the lower fungicidal rate resulted in less disease than the standard $1 \times$ treatment (Table 3.9). This was achieved because the early season applications of $0.25 \times$ mancozeb delayed epidemic onset (Fig. 3.34), reduced the height of the epidemic waves, and also slowed the epidemic rate significantly $(P=0.05)$ (Table 3.9). Delay of epidemic onset and reduction of the initial waves of disease lowered $y_{f}$ and AUDPC. This occurred even in the mixed schedule I, in 
Figure 3.34. Disease progress curves (A) and epidemic waves (B) for crown rust of oats under single- and multiple-rate schedules of fungicidal sprays in 1997. The standard (1x) rate of mancozeb was applied on days 14,25 , and 37 . The reduced $(0.25 \times)$ rate was applied 12 times (three times a week) after inoculation. The mixed schedule I [0.25×(4) $+1 \times(2)]$ included four sprays of the $0.25 \times$ rate followed by two sprays of the $1 \times$ rate. The mixed schedule II $[0.25 \times(6)+1 \times(2)]$ had six sprays of the $0.25 \times$ rate plus two sprays of the $1 \times$ rate. Arrows indicate the end of epidemic waves. The oat plants were inoculated on 15 February 1997. 


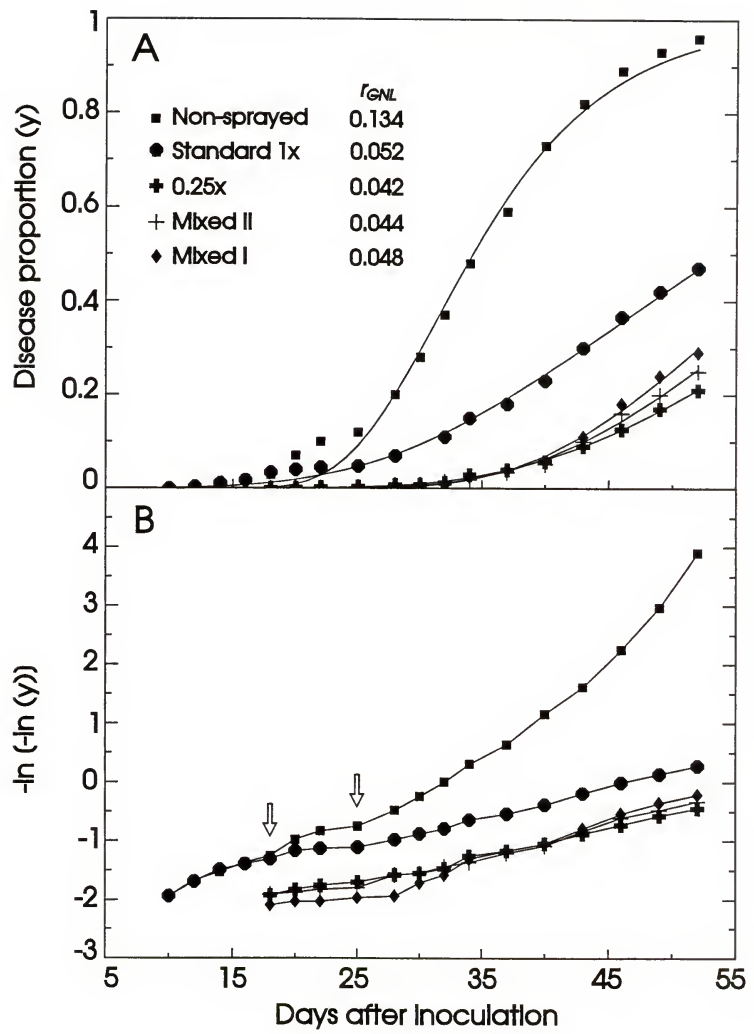


which the epidemic rate did not differ from that of the standard treatment. Although the mixed schedule I had a higher epidemic rate for crown rust than the $0.25 \times$ treatment, the total disease (AUDPC) did not differ significantly $(P=0.05)$ between the mixed-rate treatment and applications of $0.25 \times$ mancozeb alone.

Modification of the standard fungicidal schedule for oats to allow for applications of the $0.25 \times$ rate early in the season also led to higher kernel yield and better kernel quality (Table 3.10). The mixed schedule II differed $(P=0.05)$ from the standard treatment in both yield and thousand-kernel weight. The mixed schedule I had better kernel quality only. Overall, the multiple-rate programs equaled the kernel yield and kernel quality provided by season-long sprays of the $0.25 \times$ fungicidal rate.

Table 3.10: Kernel yields and thousand-kernel weights of oat plants subjected to singleand multiple-rate spray schedules of the fungicide mancozeb in 1997

\begin{tabular}{lccc}
\hline $\begin{array}{l}\text { Fungicidal } \\
\text { spray program }\end{array}$ & $\begin{array}{c}\text { Number of } \\
\text { sprays }\end{array}$ & $\begin{array}{c}\text { Yield } \\
\left(\mathbf{k g ~ h a}^{-1}\right)\end{array}$ & $\begin{array}{c}\text { Thousand-kernel } \\
\text { weight } \mathbf{~ ( g ) ~}\end{array}$ \\
\hline 0.25x & 12 & $4139 \mathrm{a}^{\mathrm{z}}$ & $28.6 \mathrm{a}$ \\
Mixed II & $6+2$ & $4411 \mathrm{a}$ & $29.1 \mathrm{a}$ \\
Mixed I & $4+2$ & $3877 \mathrm{a}$ & $27.5 \mathrm{a}$ \\
Standard $1 \times$ & 3 & $3213 \mathrm{~b}$ & $24.8 \mathrm{~b}$ \\
Nonsprayed & - & $1532 \mathrm{c}$ & $20.8 \quad \mathrm{c}$ \\
\hline P P F & & 0.0001 & 0.0001 \\
\hline
\end{tabular}

${ }^{z}$ Treatment means within columns followed by the same letter do not differ significantly $(P<$ 0.05 ) by Duncan's Multiple Range Test.

\section{Relationship of the Area Under the Disease progress Curve to Kernel Yield}

The detrimental effect of crown rust on the kernel yield of oats was studied in the 1995 and 1997 growing seasons. In 1995, two experiments were planted side-by-side and plants were subjected to similar environment and growing conditions. Data on AUDPC and kernel yield from both 1995 experiments were pooled and subjected to regression a 


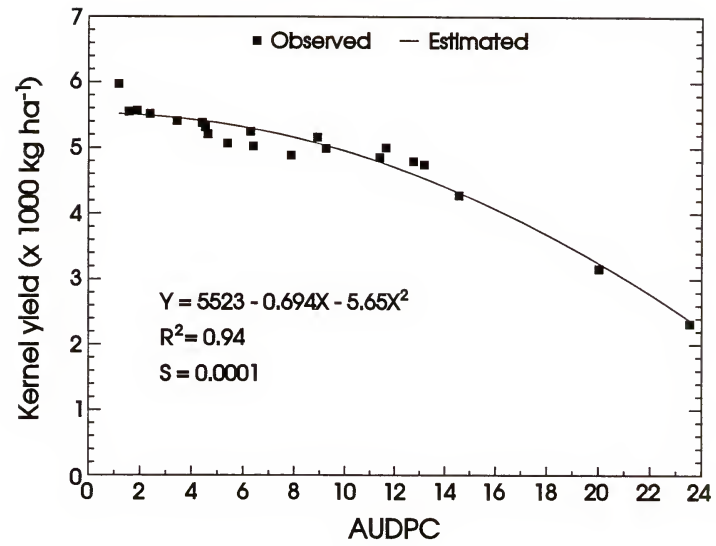

Figure 3.35. Relationship of the area under the disease progress curve (AUDPC) of crown rust to kernel yield of oats, cultivar Fulghum, in 1995. 


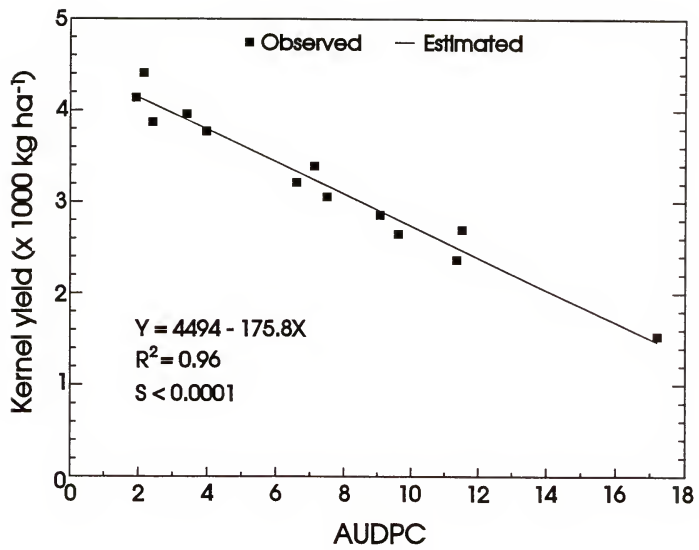

Figure 3.36. Relationship of the area under the disease progress curve (AUDPC) of crown rust to kernel yield of oats, cultivar Fulghum, in 1997. 
nalysis. The relationship of AUDPC to kernel yield (Fig. 3.35) was represented by the quadratic equation $Y=5523-0.694 X-5.65 X^{2}, R^{2}=0.94$, in which $Y$ is kemel yield in $\mathrm{kg}$ $\mathrm{ha}^{-1}$ and $X$ is AUDPC. In 1997, the analysis of the variation in yield associated with crown rust was restricted to one experiment with 13 treatments, which corresponded to several spray programs tested for the control of crown rust. In this experiment, the kemel yield diminished linearly $\left(Y=4494-175.8 X, R^{2}=0.96\right)$ as the AUDPC increased (Fig. 3.36).

\section{Discussion}

The potential of lower rates of fungicide to control epidemics of oat crown rust was demonstrated in both greenhouse and field experiments. Under controlled inoculation of $P$. coronata in the greenhouse, rates of the fungicide mancozeb as low as $0.125 \times$ and $0.25 \times$ avoided or significantly reduced infection of oat plants by the rust fungus. The inoculum density applied to oat plants in the greenhouse was as high as $4 \times 10^{4}$ uredospores $\mathrm{ml}^{-1}$ which is probably much higher than the amount of inoculum that plants are exposed to under natural conditions. Therefore, preventive applications of lower fungicidal rates can safely prevent the establishment of oat crown rust in the field.

Neely (1970) reported that residues of maneb did not prevent conidial germination of some phytopathogenic fungi one week after application. In this investigation on crown rust of oats, the disease efficiency (e) of $P$. coronata gradually increased to $0.06 \% 6$ days after the $1 \times$ rate of mancozeb was applied. This increase in efficiency occurred in direct relation to the decay of fungicidal residue on the leaf surface. In contrast, $e$ was never higher than $0.01 \%$ on plants sprayed with the $0.25 \times$ rate. The applications of the reduced rate at short intervals maintained a sufficient level of fungicide on the leaf surface to 
prevent later increase in disease. Short-spray intervals are essential to protect the developing new tissues during periods of intense plant growth and weather favorable to disease development.

Epidemics of crown rust of oats were very severe in two (1995 and 1997) of three cropping seasons in which field studies were conducted. Despite the severity of such epidemics, spray programs based on preventive applications of $0.25 \times$ or $0.5 \times$ the label rate of mancozeb delayed epidemic onset, reduced initial and total disease, and increased kernel yield significantly compared to standard sprays of the full rate of fungicide. Even when applications of lower rates did not slow the epidemic rate over the standard treatments, the AUDPC was significantly reduced. In these cases, the lower AUDPC associated with the reduced-rate treatments was totally due to fungicidal effects on the initial epidemic waves.

In a rapid epidemic, the first application of a protectant fungicide after symptoms become apparent is unlikely to be effective for commercial practice (van der Plank, 1963). The overall efficacy of the low $(0.25 x)$ and full $(1 x)$ rates of fungicide was markedly affected when spray applications were initiated after establishment of the disease in plants. The epidemic rate, the final disease, and the AUDPC increased as the spray programs were delayed until action thresholds in the range of 0.0001 to 0.05 disease severity. The delayed treatments also yielded less compared to preventive fungicidal sprays.

If the action threshold is higher than the amount of disease near the end of the first wave for a given epidemic, then the tempo of the epidemic has already been established (R. D. Berger, personal communication). In this investigation on crown rust of oats, the first epidemic wave increased the amount of disease 26 - to 30 -fold. The disease severity 
on nonsprayed plants at the end of the first epidemic wave was 0.007 in 1995 and 0.02 in 1997. Therefore, any spray program initiated beyond these disease levels would have low impact on the epidemic. Indeed, as observed in both the 1995 and 1997 experiments, applications of the $0.25 \times$ and the $1 \times$ rates according to the $0.0001-$ to 0.05 -action thresholds had no effect on the epidemic waves of crown rust. Additionally, precise detection of disease levels associated with action thresholds was complicated by latent infections and by fast progress and spread of crown rust in early epidemic stages.

Although the low-rate spray programs provided better control of crown rust and higher yields than the standard schedules, season-long sprays of reduced rates of fungicides may not be economically viable because of high application costs. To reduce the number of fungicidal applications, short-term spray schedules were tested for the control of oat crown rust. Discontinuation of fungicidal treatments at 3 or 4 weeks after inoculation, however, resulted in poorer control of crown rust than spray programs extended for 5 to 7 weeks. The disease proportion at the time fungicidal sprays have ceased in 1995 were less than 0.03 in the 3-week and less than 0.05 in the 4-week treatment. The large amount of healthy and unprotected tissue that remained presented numerous sites available for infection. The availability of healthy tissue allowed a rapid increase in disease, which reduced the benefit of fungicidal treatments. Similar examples of rapid epidemic progress after sprays of fungicide have ceased were reported elsewhere (Berger, 1975, 1977, and 1988; Keinath, 1995; van Eeckhout et al., 1991).

The rapid increase in disease discussed previously was prevented by using mixedrate spray programs. Early-season sprays of the $0.25 \times$ rate for 2 or 3 weeks delayed epidemic onset and reduced the initial amount of crown rust. Two later applications of the full rate of fungicide kept the epidemic rate slow until the end of the cropping season. 
The AUDPC and kernel yield that resulted from six or eight sprays of fungicide according to mixed-rate schedules equaled those provided by 12 applications of the $0.25 \times$ rate. Mixed-rate programs like the ones tested in this research represent an improvement of traditional standard schedules and may be of economical value for the control of plant diseases.

Crown rust of oats caused severe yield reduction in the 1995 and 1997 growing seasons. In 1997, the decrease in kernel yield associated with increase of AUDPC was linear, that is, the detrimental effect of the disease on yield was proportional to the increase in AUDPC. In 1995, the epidemics of crown rust were longer and accumulated more disease than in 1997. The relationship of disease to yield was represented by a quadratic equation, in which each increment of AUDPC caused a differential reduction on yield. Also, in the 1995 study, data from two experiments were pooled and analyzed together. In the action threshold trial, initiation of fungicidal treatments at different disease levels resulted in early accumulation of crown rust. In the experiment on duration of spray schedules, all fungicidal treatments were initiated early in the epidemic but discontinued after 3 to 7 weeks. Therefore, crown rust accumulated most at the end of the growing season and caused slightly less yield loss than early development of crown rust. Although integral models that use AUDPC cannot distinguish between early and late occurring epidemics (Campbell and Madden, 1990; Francl et al., 1997), the AUDPC explained $94 \%\left(R^{2}=0.94\right)$ of the variation in kernel yield in 1995 and $96 \%\left(R^{2}=0.96\right)$ in 1997.

In previous studies (Bissonnette et al., 1994; Forcelini et al., 1993), yield reductions of $40-50 \mathrm{~kg} \mathrm{ha}^{-1}$ for each percent increase in crown rust up to $30 \%$ disease were reported. The above studies were developed using single-point models which took 
into account the amount of disease at the growth stage 75 (11.1 in the Feekes scale) (Bissonnette et al., 1994) or at the end of the epidemic (Forcelini et al., 1993). In our research on crown rust, we used AUDPC-based models to analyze epidemics whose proportions of final disease were over 0.96 . For such reasons, we were unable to compare our results to previous reports on the effects of crown rust on oat yield. 


\section{CHAPTER IV \\ MANAGEMENT OF EPIDEMICS OF \\ HELMINTHOSPORIUM LEAF SPOT ON RYE}

\section{Introduction}

Rye (Secale cereale L.) is a minor crop in the U.S. and little information is available regarding the control of its diseases. In Florida, rye plants may be affected by anthracnose, helminthosporium leaf spot, leaf rust, seedling blights, pythium root rot, and common root rots (Kucharek, 1994b). Among these diseases, helminthosporium leaf spot (HLS), caused by Bipolaris sorokiniana (teleom. Cochliobolus sativus), is the most common in the field. Although this disease can affect the roots and all above-ground plant parts, the football-shaped, reddish-brown lesions on leaves are the predominant symptoms of HLS (Kucharek, 1994b).

Control of HLS in rye is usually obtained using disease-free or treated seeds and crop rotation (Kucharek, 1994b). Although HLS may reach high intensity levels in certain years, the low value of the rye crop has precluded the use of fungicides for disease control. Bipolaris sorokiniana, however, has a wide host range and affects more important cereal crops such as barley and wheat (Kucharek, 1988), for which crops fungicide treatments may be recommended (Kucharek 1994c). Because rye is very susceptible to HLS, this crop was used as a model system to study the management of HLS epidemics on cereal crops with reduced rates of fungicides. 


\section{Material and Methods}

Experiments on the rye-HLS pathosystem were conducted during the 1996 and 1997 cropping seasons in a field area located southwest of Lake Alice, in Gainesville, FL. The cultivar Wrens Abruzzi, susceptible to HLS, was used in both years. The land preparation, fertilization, experimental design, planting and growing procedures, spray equipment, disease assessment, and statistical analyses were similar to those previously described for the experiments on oats. Also, analyses of the disease progress curves and epidemic parameters followed the methodology described in the previous chapter. In both the 1996 and 1997 experiments, the rye plants were not inoculated since the crop was grown in an area planted to rye the previous two years and the seeds used for planting were naturally infected by $B$. sorokiniana at an incidence level of $18 \%$. Seed infection by the fungus was determined after incubation of 400 rye seeds on wet filter paper for 7 days at $23-25^{\circ} \mathrm{C}$.

\section{Experiment}

This experiment was established on 8 December 1995. Ten fungicidal programs, each one replicated five times, were tested for the control of HLS epidemics. The Florida disease-control program for wheat (Kucharek and Luke, 1989; Kucharek, 1994c) was adapted to rye and used as the standard program for chemical control of HLS. This program is based on three applications of mancozeb (Manzate $200 \mathrm{DF}$ ), at $1.8 \mathrm{~kg}$ a.i. ha-1, one at the plant growth stage 32 (Zadoks et al., 1974) (7 in the Feekes scale; Large, 1954) and two others at 10 - to 14 -day intervals. In this experiment, the standard $1 \times$ rate, applied at 91 (8 March 1996) (growth stage 32), 103 (20 March 1996), and 114 days (31 
March 1996) after planting, was compared to six weekly sprays of $0.5 \times$ and twelve semiweekly sprays of $0.25 \times$ rates initiated 84 days (1 March 1996) after planting. The $0.25 \times$ treatment was then compared to several other fungicidal schedules, that is, $i$, a delay treatment (0.25x-delay) initiated 91 days (8 March 1996) after planting and reapplied 10 times at semiweekly intervals, $i i$, two short-term schedules, begun 84 days (1 March 1996) after planting, repeated semiweekly, but discontinued after four (Short I) or six (Short II) sprays, iii, and three mixed schedules, which began on day 84 (1 March 1996) and combined four sprays of the $0.25 \times$ rate plus two applications of the $1 \times$ rate (Mixed I), four sprays of the $0.25 \times$ rate plus four applications of the $0.5 \times$ rate (Mixed II), and six sprays of the $0.25 \times$ rate plus three applications of the $0.5 \times$ rate of mancozeb (Mixed III). The rye plants were harvested and thrashed on 27 April 1996 to determine kernel yield.

\section{Experiment}

This experiment was planted on 11 December 1996 following a complete randomized block design with eight treatments and four replicates each. The fungicidal spray programs tested in the 1996 experiment, excluding the short-term schedules Short I and Short II, were reevaluated in 1997. The number of applications and the spray intervals used in 1996 were maintained in 1997. In this experiment, the standard 1x treatment was applied at 82 (3 March 1997) (growth stage 32), 93 (14 March 1997), and 105 days (26 March 1997) after planting. The $0.25 \times$-delay schedule began on day 82 (3 March 1997). The $0.25 x-$ and $0.5 x$-rate schedules, as well as the mixed spray programs, were initiated 68 days (17 February 1997) after planting. The rye plants were harvested on 7 May 1997. 


\section{$\underline{\text { Results }}$}

Because the field plots were established in an area traditionally planted to the crop, and because $18 \%$ of the rye seeds planted were infected by $B$. sorokiniana, HLS appeared early and evenly distributed in the field in both the 1996 and 1997 growing seasons. The early disease occurrence affected most the old basal leaves, which became diseased before any fungicidal treatment was applied. To account for this problem, disease severity was assessed on the four upper leaves (Flag, F-1, F-2, and F-3 leaves) of the main tiller of the sample plants.

Despite the early occurrence of HLS, the low temperatures recorded in February and March 1996 limited the development of the disease on the upper four leaves until 98 days (16 March 1996) after planting $\left(y_{0}=0.005\right)$ (Fig. 4.1-A). After day 98 (16 March 1996), HLS progressed at an epidemic rate of 0.045 gompits day $^{-1}$ in nonsprayed plants and reached its maximum severity (0.362) 36 (21 April 1996) days later. The AUDPC for this epidemic was 5.03 .

The estimated daily increments of disease severity $\left(d_{y} / d_{t}\right)$ (Fig. 4.1-B) were lower than 0.005 before 112 days (30 March 1996) after planting but as high as 0.016 at days 118 (5 April 1996) and 127 (14 April 1996). The major disease increase on days 118 (5 April 1996) and 127 (14 April 1996) resulted from rainfall and disease-favorable temperatures observed one latent period before, that is, on days 100 (18 March 1996) and 110 (28 March 1996). After day 125 (12 April 1996), $d_{y} / d_{t}$ decreased gradually as the availability of susceptible host tissue had reduced.

Transformation of disease proportions by $-\ln [-\ln (y)]$ resulted in nearly a straight line, with only a flat epidemic wave (Fig. 4.2), which occurred between days 98 (16 


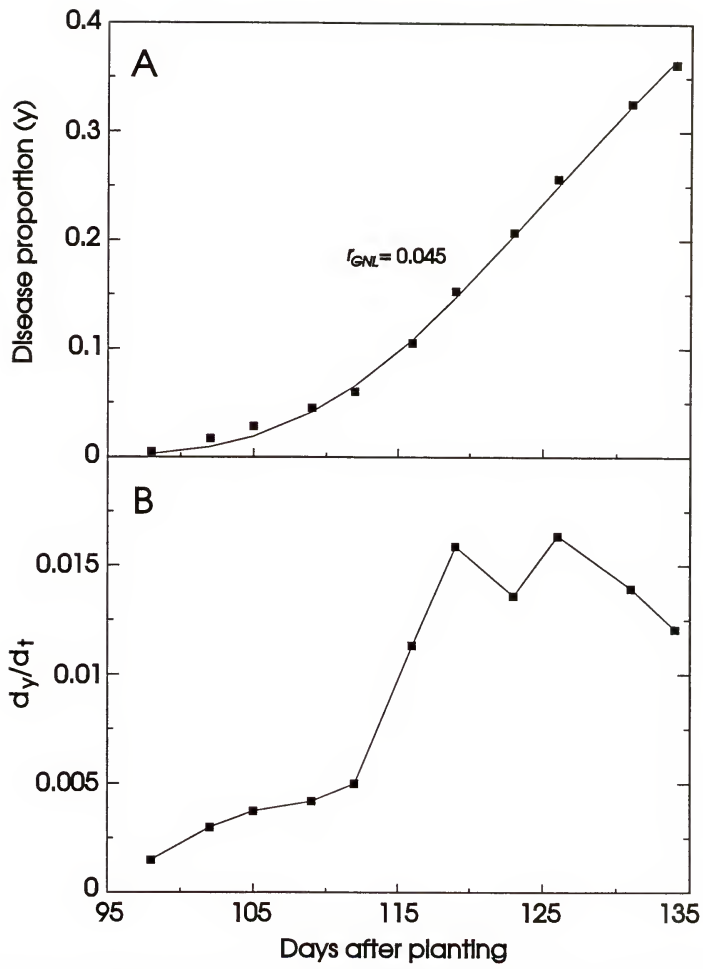

Figure 4.1. Disease-progress curve (A) and estimated daily increment of disease severity $\left(d_{y} / d_{t}\right)$ (B) for helminthosporium leaf spot on nonsprayed rye plants in 1996. The epidemic rate was calculated as $r_{G}=$ [gompit $\left(y_{f}\right)$ - gompit $\left.\left(y_{0}\right)\right] / \Delta t$, where gompit is - $\ln [-$ $\ln (y)], y_{0}$ and $y_{f}$ are the initial and the final disease proportions, and $\Delta t$ is the time interval between $t_{0}$ and $t_{f}$ 


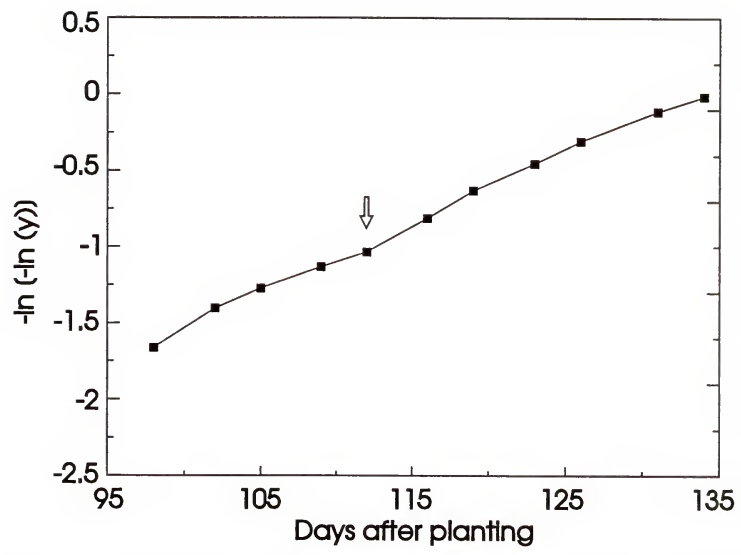

Figure 4.2. Disease wave for an epidemic of helminthosporium leaf spot on nonsprayed rye plants in 1996. The epidemic wave was obtained transforming the disease proportions by $-\ln [-\ln (y)]$. The arrow indicates the end of the epidemic wave. 
March 1996) and 113 (31 March 1996). During this epidemic wave, the disease proportion of HLS increased from 0.005 to 0.06 .

In 1997, the HLS epidemic in nonsprayed plants (Fig. 4.3-A) occurred earlier (day 75,24 February 1997), developed faster $\left(r_{G N L}=0.064\right.$ gompits day $\left.{ }^{-1}\right)$, and reached a final disease severity $\left(y_{f}=0.58\right)$ higher than in 1996. The AUDPC was 8.18. The $d_{y} / d_{t}$ values (Fig. 4.3-B) were maximum (>0.02) between days 98 (19 March 1997) and 109 (30 March 1997). The plot of disease values transformed by $-\ln [-\ln (y)]$ (Fig. 4.4) again had one epidemic wave, which lasted about 13 days and corresponded to an 8-fold (from 0.01 to 0.082 ) disease increase.

\section{Effects of Low Fungicidal Rates on Epidemic Parameters and Rye Yield}

In both the 1996 and 1997 experiments on rye, two reduced-rate programs $(0.25 \times$ and $0.5 \times$ the label rate of mancozeb) of fungicidal sprays were tested for the control of HLS. The lower rates were applied weekly $(0.5 x)$ or twice-weekly $(0.25 x)$ and compared to a standard $(1 \times)$ treatment sprayed three times in each growing season. In both years, the $0.25 \times$ rate was sprayed 12 times and the $0.5 \times$ rate six times. Disease assessments were initiated one latent period (12 to 14 days) after the first fungicidal spray. The disease progress curves and disease waves for the HLS epidemics from these experiments are shown in Figures 4.5 and 4.6.

Since the rye plants were naturally infected prior to the initiation of the spray programs, applications of fungicide were not able to delay epidemic onset. The major effect of the early sprays was to reduce the amount of initial disease $\left(y_{0}\right)$ on the four uppermost leaves. The $y_{0}$ ranged from $0.001(0.25 \times)$ to $0.002(0.5 \times)$, and to 0.005 (nonsprayed plants) (Table 4.1 and Fig. 4.5-B). The standard treatment, initially sprayed 


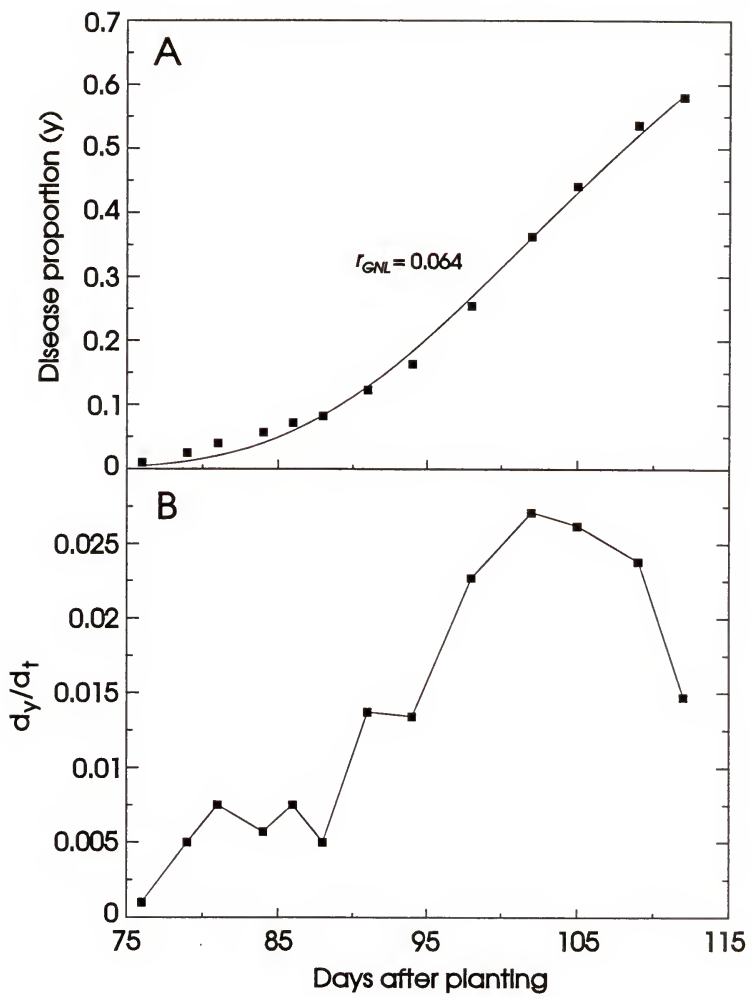

Figure 4.3. Disease-progress curve (A) and estimated daily increment of disease severity $\left(d_{y} / d_{t}\right)$ (B) for helminthosporium leaf spot on nonsprayed rye plants in 1997. The epidemic rate was calculated as $r_{G}=$ [gompit $\left(y_{f}\right)$ - gompit $\left.\left(y_{0}\right)\right] / \Delta t$, where gompit is - $\ln [-$ $\ln (y)], y_{0}$ and $y_{f}$ are the initial and the final disease proportions, and $\Delta t$ is the time intervals between $t_{0}$ and $t_{f}$ 


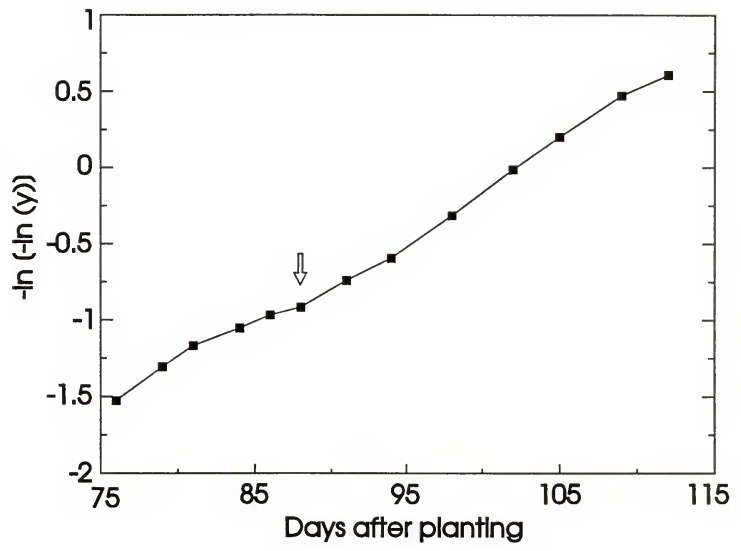

Figure 4.4. Disease wave for an epidemic of helminthosporium leaf spot on nonsprayed rye plants in 1997. The epidemic wave was obtained transforming the disease proportions by $-\ln [-\ln (y)]$. The arrow indicates the end of the epidemic wave. 
Figure 4.5. Disease progress curves (A) and epidemic waves (B) for helminthosporium leaf spot on rye plants sprayed with full and lower rates of mancozeb in 1996. The $0.25 \times$ and $0.5 \times$ rates were sprayed 12 (twice-weekly) and six (weekly) times from day 84 after planting. The standard $1 \times$ treatment was applied on days 91 (growth stage 32 in the decimal code scale; 7 in the Feekes scale), 103, and 114. The epidemic rate was calculated as $r_{G}=$ [gompit $\left(y_{f}\right)$ - gompit $\left.\left(y_{0}\right)\right] / \Delta t$, where gompit is $-\ln [-\ln (y)], y_{0}$ and $y_{f}$ are the initial and the final disease proportions, and $\Delta t$ is the time intervals between $t_{0}$ and $t_{f}$ The epidemic wave shown in B was obtained transforming the disease proportions by - $\mathrm{ln}$ $[-\ln (y)]$. The arrow indicates the end of the epidemic wave. 


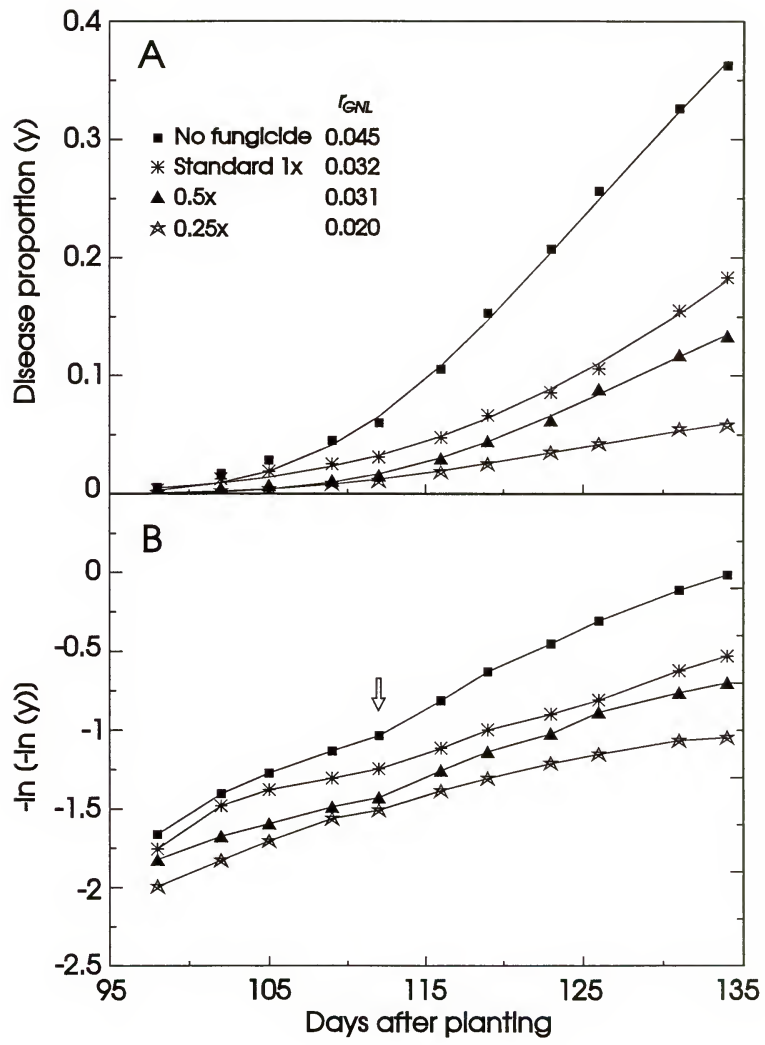


plants subjected to the $0.25 \times$-spray program had the lowest final disease $\left(y_{f}=0.06\right)$ and AUDPC (0.84) in 1996.

Table 4.2. Initial $\left(y_{0}\right)$ and final $\left(y_{f}\right)$ disease proportions, estimated epidemic rates $\left(r_{G N L}\right)$, and areas under the disease progress curves (AUDPC) for epidemics of helminthosporium leaf spot on rye plants sprayed with various rates of the fungicide mancozeb in 1997. The proportions of disease severity were assessed on flag, $f-1, f-2$, and $\mathrm{f}-3$ leaves

\begin{tabular}{lcccc}
\hline & \multicolumn{5}{c}{ Epidemic parameters } \\
\cline { 2 - 5 } Spray program $^{\mathrm{x}}$ & $\boldsymbol{y}_{0}$ & $\boldsymbol{y}_{f}$ & $\boldsymbol{r}_{G N L}{ }^{\mathrm{y}}$ & AUDPC \\
\hline $0.25 \times$ & $0.003 \mathrm{a}^{2}$ & $0.12 \mathrm{a}$ & $0.027 \mathrm{a}$ & $1.38 \mathrm{a}$ \\
$0.5 \times$ & $0.005 \mathrm{a}$ & $0.15 \mathrm{a}$ & $0.029 \mathrm{a}$ & $1.84 \mathrm{a}$ \\
Standard 1× & $0.01 \mathrm{~b}$ & $0.29 \mathrm{~b}$ & $0.039 \mathrm{~b}$ & $3.95 \mathrm{c}$ \\
Nonsprayed & $0.01 \mathrm{~b}$ & $0.58 \mathrm{c}$ & $0.064 \mathrm{c}$ & $8.18 \mathrm{~d}$ \\
\hline$P>F$ & 0.015 & 0.0001 & 0.0001 & 0.0001 \\
\hline
\end{tabular}

${ }^{x}$ The $0.25 \times$ fungicidal rate was sprayed 12 times (twice-weekly) and the $0.5 \times$ rate six times (weekly) from day 68 after planting. The standard $1 \times$ treatment was applied three times on days 82,93 , and 105 .

${ }^{y}$ Epidemic rates were estimated by nonlinear regression of disease proportions against time using the Gompertz model.

${ }^{2}$ Treatment means within columns followed by the same letter do not differ significantly $(P>$ 0.05 ) by Duncan's Multiple Range Test, using the variability pooled among replicates. Even though rate of fungicide is a quantitative factor, the individual rates were applied at different intervals and therefore the treatments were considered unique.

In 1997, the spray programs based on applications of the $0.25 x$ and $0.5 \times$ fungicidal rates reduced $y_{0}$, slowed $r_{G N}$, and decreased $y_{f}$ and AUDPC significantly $(P=$ 0.05 ) in relation to the standard $(1 \times)$ treatment (Table 4.2 and Fig. 4.6). The control of HLS provided by the lower rates, especially 0.5x, was even better in 1997 than in 1996. Overall, the $0.25 \times$ and $0.5 \times$-spray schedules did not differ from each other on the control of HLS in 1997. 
Figure 4.6. Disease progress curves (A) and epidemic waves (B) for helminthosporium leaf spot on rye plants sprayed with full and lower rates of mancozeb in 1997. The $0.25 \times$ and $0.5 \times$ rates were sprayed 12 (twice-weekly) and six (weekly) times from day 68 after planting. The standard $1 \times$ treatment was applied on days 82 (growth stage 32 in the decimal code scale; 7 in the Feekes scale), 93, and 105. The epidemic rate was calculated as $r_{G}=$ [gompit $\left(y_{f}\right)$ - gompit $\left.\left(y_{0}\right)\right] / \Delta t$, where gompit is $-\ln [-\ln (y)], y_{0}$ and $y_{f}$ are the initial and the final disease proportions, and $\Delta t$ is the time intervals between $t_{0}$ and $t_{f}$ The epidemic wave shown in B was obtained transforming the disease proportions by $-\ln [-\ln$ $(v)]$. The arrow indicates the end of the epidemic wave. 


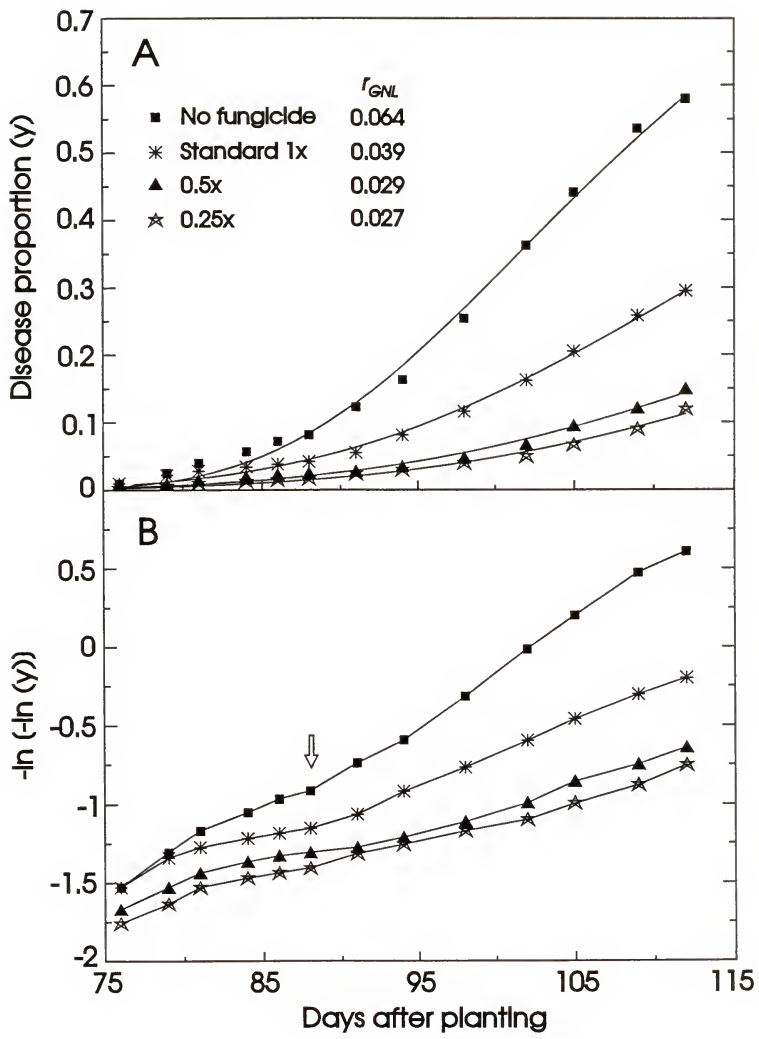


at growth stage 32 , that is, 7 days after the first application of the lower rate, did not reduce $y_{0}$.

Table 4.1. Initial $\left(y_{0}\right)$ and final $\left(y_{f}\right)$ disease proportions, estimated epidemic rates $\left(r_{G N L}\right)$, and areas under the disease progress curves (AUDPC) for epidemics of helminthosporium leaf spot on rye plants sprayed with various rates of the fungicide mancozeb in 1996. The proportions of disease severity were assessed on flag, $f-1, f-2$, and $\mathrm{f}-3$ leaves

\begin{tabular}{|c|c|c|c|c|}
\hline \multirow[b]{2}{*}{ Spray program ${ }^{x}$} & \multicolumn{4}{|c|}{ Epidemic parameters } \\
\hline & $y_{0}$ & $y_{f}$ & $r_{G N L}^{y}$ & AUDPC \\
\hline $0.25 x$ & $0.001 \mathrm{a}^{2}$ & $0.06 \mathrm{a}$ & $0.02 \mathrm{a}$ & $0.84 \mathrm{a}$ \\
\hline $0.5 x$ & $0.002 \mathrm{a}$ & $0.13 \mathrm{~b}$ & $0.031 \mathrm{~b}$ & $1.64 \mathrm{~b}$ \\
\hline Standard 1x & $0.004 \mathrm{~b}$ & $0.18 \mathrm{c}$ & $0.032 \mathrm{~b}$ & $2.32 \mathrm{c}$ \\
\hline Nonsprayed & $0.005 \mathrm{~b}$ & $0.36 \mathrm{~d}$ & $0.045 \mathrm{c}$ & $5.03 \mathrm{~d}$ \\
\hline$P>F$ & 0.001 & 0.0001 & 0.0001 & 0.0001 \\
\hline \multicolumn{5}{|c|}{$\begin{array}{l}\times \text { The } 0.25 \times \text { fungicidal rate was sprayed } 12 \text { times (twice-weekly) and the } 0.5 \times \text { six times (weekly) } \\
\text { from day } 84 \text { after planting. The standard } 1 \times \text { treatment was applied three times on days } 91,103 \text {, } \\
\text { and } 114 \text {. } \\
\text { y Epidemic rates were estimated by nonlinear regression of disease proportions against time using } \\
\text { the Gompertz model. } \\
\text { ' Treatment means within columns followed by the same letter do not differ significantly }(P> \\
\text { 0.05) by Duncan's Multiple Range Test, using the variability pooled among replicates. Even } \\
\text { though rate of fungicide is a quantitative factor, the individual rates were applied at different } \\
\text { intervals and therefore the treatments were considered unigue. }\end{array}$} \\
\hline
\end{tabular}

Reduction of the standard fungicidal rate $(1 \times)$ to $0.5 \times$ to allow for weekly applications of the latter rate did not slow $r_{G N L}$ significantly. Despite this, the $0.5 \times$ schedule resulted in lower final disease $\left(y_{f}=0.13\right)$ and lower AUDPC (1.64) than the standard $1 \times$ treatment, which had $y_{f}=0.18$ and AUDPC $=2.32$ (Table 4.1). The better control of HLS observed on plants sprayed with $0.5 \times$ mancozeb was associated with the reduction in $y_{0}$ provided by early sprays of fungicide.

Sprays of the $0.25 \times$ rate improved the control of HLS two ways. The early applications of fungicide reduced the initial disease $\left(y_{0}=0.001\right)$ while the short spray intervals accounted for slower epidemic rates $\left(r_{G N L}=0.02\right.$ gompits day $\left.{ }^{-1}\right)$. Consequently, 


\section{Effects of Late Initiation of Fungicidal Treatments}

A delay treatment ( $0.25 \times$-delay) was included in both the 1996 and 1997 experiments to evaluate the effect of late fungicidal application on the management of HLS. The delay program began at the plant growth stage 32 (7 in the Feekes scale), which is the growth stage recommended for initiation of the standard $1 \times$ treatment. The delay time between the $0.25 \times$ treatment and $0.25 \times$-delay was 7 days in 1996 and 14 days in 1997.

Table 4.3. Initial $\left(y_{0}\right)$ and final $\left(y_{f}\right)$ disease proportions, estimated epidemic rates $\left(r_{G N L}\right)$, and areas under the disease progress curves (AUDPC) for epidemics of helminthosporium leaf spot on rye plants subjected to early and late sprays of a low rate of the fungicide mancozeb in 1996. The proportions of disease severity were assessed on flag, $\mathrm{f}-1, \mathrm{f}-2$, and $\mathrm{f}-3$ leaves

\begin{tabular}{|c|c|c|c|c|}
\hline \multirow[b]{2}{*}{ Spray program ${ }^{x}$} & \multicolumn{4}{|c|}{ Epidemic parameters } \\
\hline & $y_{0}$ & $y_{f}$ & $\boldsymbol{r}_{G N L}{ }^{\mathbf{y}}$ & AUDPC \\
\hline $0.25 x$ & $0.001 a^{z}$ & $0.06 \mathrm{a}$ & $0.02 \mathrm{a}$ & $0.84 \mathrm{a}$ \\
\hline $0.25 x$-delay & $0.004 \mathrm{~b}$ & $0.21 \mathrm{~b}$ & $0.034 \mathrm{~b}$ & $2.48 \mathrm{~b}$ \\
\hline Standard $1 \times$ & $0.004 \mathrm{~b}$ & $0.18 \mathrm{~b}$ & $0.032 \mathrm{~b}$ & $2.32 \mathrm{~b}$ \\
\hline Nonsprayed & $0.005 \mathrm{~b}$ & $0.36 \mathrm{c}$ & $0.045 \mathrm{c}$ & $5.03 \mathrm{c}$ \\
\hline$P>F$ & 0.001 & 0.0001 & 0.0001 & 0.0001 \\
\hline \multicolumn{5}{|c|}{$\begin{array}{l}\text { The early-spray program }(0.25 \times) \text { was applied } 12 \text { times (twice-weekly) from day } 84 \text { after } \\
\text { planting. The delay treatment }(0.25 \times \text {-delay) was sprayed } 10 \text { times (twice-weekly) from day } 91 \text {. } \\
\text { The standard } 1 \times \text { rate of mancozeb was applied three times on days } 91,103 \text {, and } 114 \text {. } \\
{ }^{y} \text { Epidemic rates were estimated by nonlinear regression of disease proportions against time using } \\
\text { the Gompertz model. } \\
\text { x } \\
\text { Treatment means within columns followed by the same letter do not differ significantly }(P> \\
0.05) \text { by Duncan's Multiple Range Test, using the variability pooled among replicates. Even } \\
\text { though rate of fungicide is a quantitative factor, the individual rates were applied at different } \\
\text { intervals and therefore the treatments were considered unique. }\end{array}$} \\
\hline
\end{tabular}

Delay of spray applications until growth stage 32 affected the efficiency of the fungicidal treatment (Fig. 4.7 and 4.8). In both experiments, the $y_{0}, r_{G N L}, y_{f}$ and AUDPC associated with the $0.25 \times$-delay treatment were significantly higher $(P=0.05)$ than those 
observed for the early sprays of the same fungicidal rate $(0.25 \times)$ (Tables 4.3 and 4.4$)$. The late application of fungicide ( $0.25 \times$-delay) also resulted in epidemic waves (Fig. 4.7B and 4.8-B) whose initial and final disease levels exceeded those of the early treatment $(0.25 \times$ rate $)$.

In the 1996 and 1997 experiments, the delay program behaved like the standard $1 \times$ treatment (Fig 4.7-A and 4.8-A), that is, the $0.25 \times$-delay did not differ from the $1 \times$ rate in any of the epidemic parameters analyzed $\left(y_{0}, y_{f}, r_{G N L}\right.$, and AUDPC). The epidemic waves (Fig. 4.7-B and 4.8-B) for $0.25 \times$-delay and $1 \times$ treatments also had similar initial and final disease levels. Therefore, the late initiation of the spray applications precluded the lower fungicidal rate of improving the management of HLS epidemics.

Table 4.4. Initial $\left(y_{0}\right)$ and final $\left(y_{f}\right)$ disease proportions, estimated epidemic rates $\left(r_{G N L}\right)$, and areas under the disease progress curves (AUDPC) for epidemics of helminthosporium leaf spot on rye plants subjected to early and late sprays of a low rate of the fungicide mancozeb in 1997. The proportions of disease severity were assessed on flag, $\mathrm{f}-1, \mathrm{f}-2$, and $\mathrm{f}-3$ leaves

\begin{tabular}{l}
\multicolumn{5}{c}{ Epidemic parameters } \\
\cline { 2 - 5 } Spray program \\
\cline { 2 - 5 }
\end{tabular}


Figure 4.7. Disease progress curves (A) and epidemic waves (B) for helminthosporium leaf spot on rye plants subjected to early and late sprays of $0.25 \times$ the label rate of mancozeb in 1996. The early spray program $(0.25 \times)$ was applied 12 times (twiceweekly) from day 84 after planting. The delay treatment $(0.25 \times$-delay) was sprayed 10 times (twice-weekly) from day 91 (growth stage 32 in the decimal code scale; 7 in the Feekes scale). The standard $1 \times$ treatment was applied on days 91 (growth stage 32), 103, and 114. The epidemic rate was calculated as $r_{G}=$ [gompit $\left(y_{f}\right)$ - gompit $\left(y_{0}\right)$ ] / $\Delta t$, where gompit is $-\ln [-\ln (y)], y_{0}$ and $y_{f}$ are the initial and the final disease proportions, and $\Delta t$ is the time intervals between $t_{0}$ and $t_{f}$ The epidemic wave shown in B was obtained transforming the disease proportions by $-\ln [-\ln (y)]$. The arrow indicates the end of the epidemic wave. 


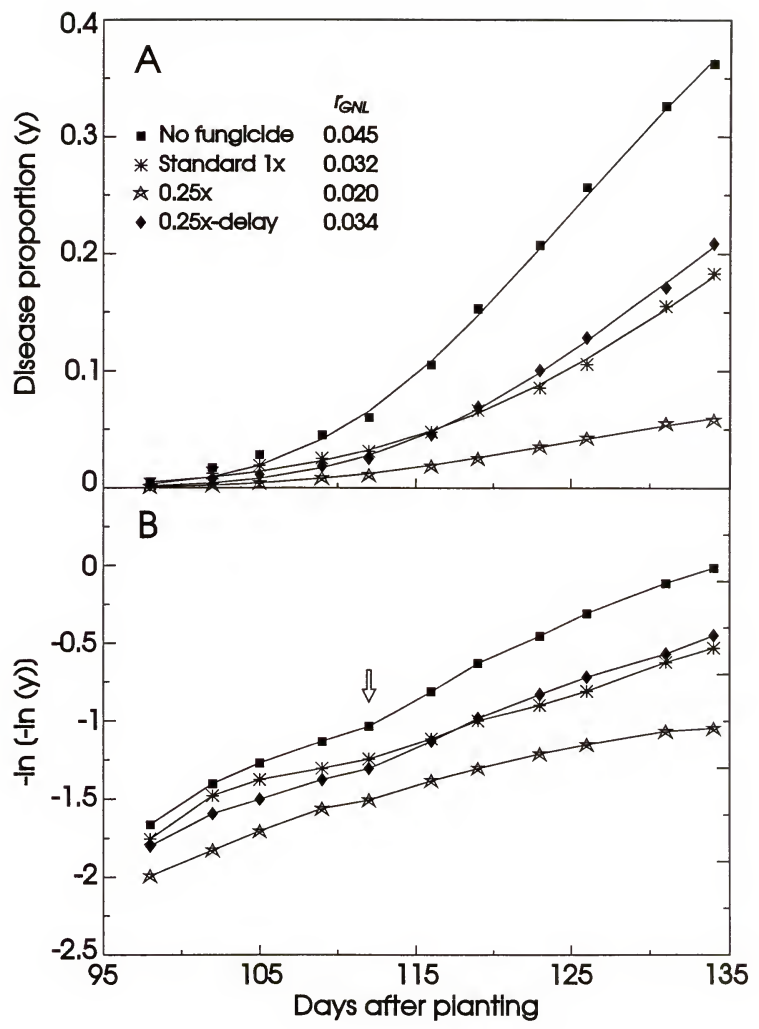


Figure 4.8. Disease progress curves (A) and epidemic waves (B) for helminthosporium leaf spot on rye plants subjected to early and late sprays of $0.25 \times$ the label rate of mancozeb in 1997 . The early spray program $(0.25 \times)$ was applied 12 times (twiceweekly) from day 68 after planting. The delay treatment $(0.25 \times$-delay $)$ was sprayed 10 times (twice-weekly) from day 82 (growth stage 32 in the decimal code scale; 7 in the Feekes scale). The standard $1 \times$ treatment was applied on days 82 (growth stage 32), 93, and 105. The epidemic rate was calculated as $r_{G}=$ [gompit $\left(y_{f}\right)$ - gompit $\left.\left(y_{0}\right)\right] / \Delta t$, where gompit is $-\ln [-\ln (y)], y_{0}$ and $y_{f}$ are the initial and the final disease proportions, and $\Delta t$ is the time intervals between $t_{0}$ and $t_{f}$ The epidemic wave shown in B was obtained transforming the disease proportions by $-\ln [-\ln (y)]$. The arrow indicates the end of the epidemic wave. 


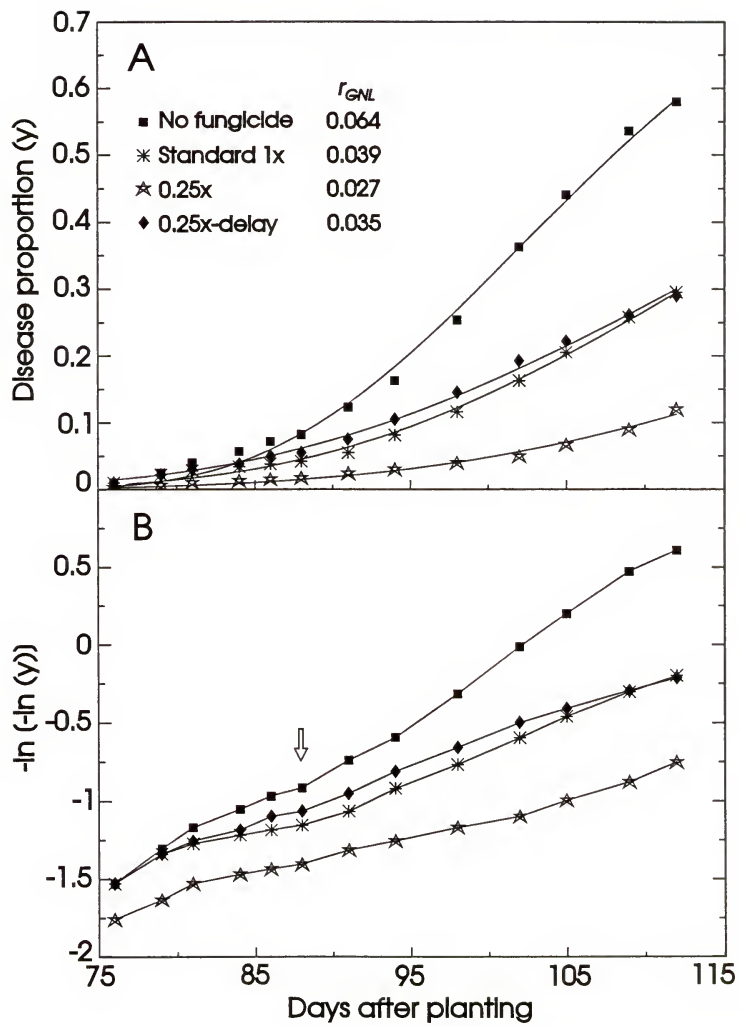




\section{Effects of Discontinued Fungicidal Treatments}

In 1996, two short-term programs of fungicidal sprays were initiated 84 days (2 March 1996) after planting, repeated twice-weekly for 2 (Short I) or 3 weeks (Short II) and then discontinued. The disease progress that followed the loss of fungicidal effectiveness was evaluated and compared to season-long sprays of $0.25 \times$ and $1 \times$ mancozeb.

Table 4.5. Initial $\left(y_{0}\right)$ and final $\left(y_{f}\right)$ disease proportions, estimated epidemic rates $\left(r_{G N L}\right)$, and areas under the disease progress curves (AUDPC) associated with epidemics of helminthosporium leaf spot on rye plants subjected to short-term spray schedules of the fungicide mancozeb in 1996. The proportions of disease severity were assessed on flag, $\mathrm{f}-1, \mathrm{f}-2$, and $\mathrm{f}-3$ leaves

\begin{tabular}{|c|c|c|c|c|}
\hline \multirow[b]{2}{*}{ Spray program ${ }^{x}$} & \multicolumn{4}{|c|}{ Epidemic parameters } \\
\hline & $y_{0}$ & $y_{f}$ & $\boldsymbol{r}_{G N L}$ & AUDPC \\
\hline $0.25 x$ & $0.001 \mathrm{a}$ & $0.06 \mathrm{a}$ & $0.02 \mathrm{a}$ & $0.84 \mathrm{a}$ \\
\hline Standard $1 \times$ & $0.004 \mathrm{~b}$ & $0.18 \mathrm{~b}$ & $0.032 \mathrm{~b}$ & $2.32 \mathrm{~b}$ \\
\hline Short II & $0.001 \mathrm{a}$ & $0.22 \mathrm{~b}$ & $0.042 \mathrm{c}$ & $2.26 \mathrm{~b}$ \\
\hline Short I & $0.001 \mathrm{a}$ & $0.36 \mathrm{c}$ & $0.051 \mathrm{~d}$ & $3.98 \mathrm{c}$ \\
\hline Nonsprayed & $0.005 \mathrm{~b}$ & $0.36 \mathrm{c}$ & $0.045 \mathrm{c}$ & $5.03 \mathrm{~d}$ \\
\hline$P>F$ & 0.001 & 0.0001 & 0.0001 & 0.0001 \\
\hline \multicolumn{5}{|c|}{$\begin{array}{l}{ }^{x} \text { The short-term spray programs, initiated } 84 \text { days after planting and repeated twice-weekly for } 2 \\
\text { (Short I) or } 3 \text { weeks (Short II), were compared to } 12 \text { sprays (twice-weekly) of the } 0.25 \times \text { rate } \\
\text { from day } 84 \text { and three sprays of the standard } 1 \times \text { treatment on days } 91,103 \text {, and } 114 \text {. } \\
\text { y Epidemic rates were estimated by nonlinear regression of disease proportions against time using } \\
\text { the Gompertz model. } \\
\text { x } \\
\text { Treatment means within columns followed by the same letter do not differ significantly }(P> \\
0.05 \text { ) by Duncan's Multiple Range Test, using the variability pooled among replicates. Even } \\
\text { though rate of fungicide is a quantitative factor, the individual rates were applied at different } \\
\text { intervals and therefore the treatments were considered unique. }\end{array}$} \\
\hline
\end{tabular}

The shape of the epidemic waves (Fig. 4.9-B) was affected by duration of spray programs. Sprays of mancozeb for only 2 weeks (Short I) resulted in an irregular epidemic wave, which had initial disease similar to the $0.25 \times$ rate but much higher final disease. Indeed, the amount of disease at the end of the epidemic wave for the Short I 
Figure 4.9. Disease progress curves (A) and epidemic waves (B) for helminthosporium leaf spot on rye plants subjected to short-term sprays of $0.25 \times$ mancozeb in 1996. The short-term treatments were initiated 84 days after planting, repeated twice-weekly, and discontinued after four (Short I) or six applications (Short II). Short I and II were compared to 12 sprays of $0.25 \times$ from day 84 and three applications of $1 \times$ on days 91 (growth stage 32 in the decimal code scale; 7 in the Feekes scale), 103, and 114. The epidemic rate was calculated as $r_{G}=$ [gompit $\left(y_{f}\right)$ - gompit $\left(y_{0}\right)$ ] $/ \Delta t$, where gompit is - $\ln [-$ $\ln (y)], y_{0}$ and $y_{f}$ are the initial and the final disease proportions, and $\Delta t$ is the time intervals between $t_{0}$ and $t_{f}$ The epidemic wave shown in B was obtained transforming the disease proportions by $-\ln [-\ln (y)]$. The arrow indicates the end of the epidemic wave. 


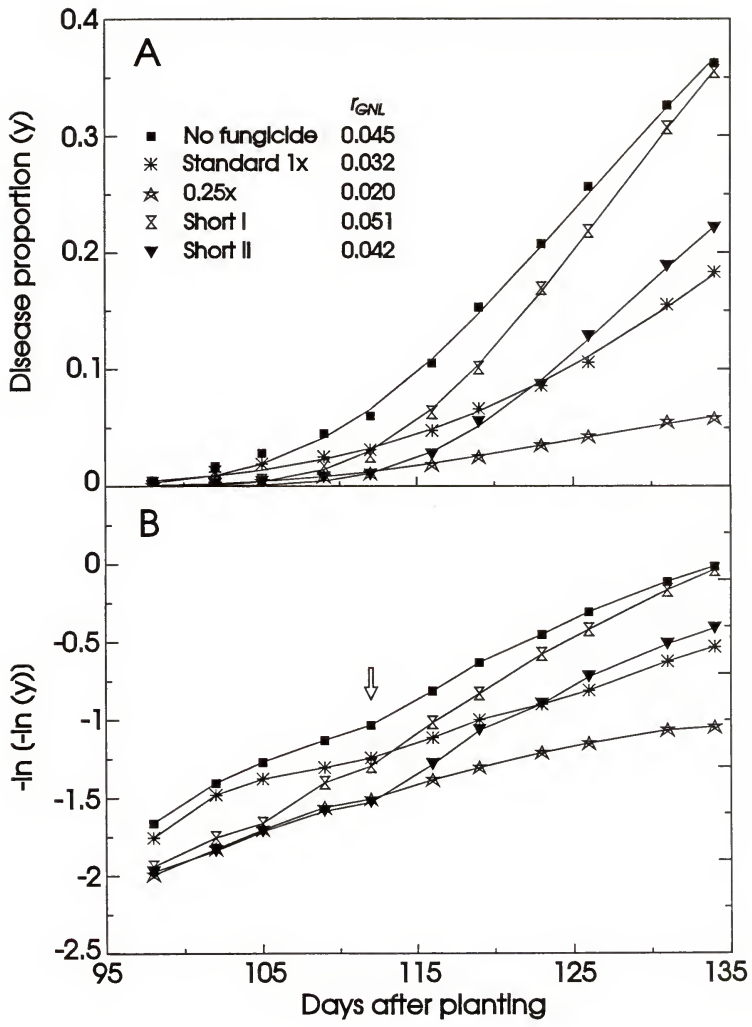


treatment was as high as that of the standard $1 \times$ rate. When the period of fungicidal sprays was extended for 3 weeks (Short II), no differences on the shape of the epidemic waves were observed between Short II and the $0.25 \times$ treatment.

The epidemics increased faster after fungicidal applications had ceased. The epidemic rates for HLS on plants subjected to short-term treatments were similar to or even higher than that of nonsprayed plants (Table 4.5). The epidemic curve and the final amount of disease for Short I approached those of the control treatment (Fig. 4.9-A). The AUDPC was higher in Short I than in the standard $1 \times$ treatment. Application of the $0.25 \times$ rate for 3 weeks (Short II) resulted in final amount of disease (Fig. 4.9-A) and AUDPC (Table 4.5) comparable to those of the standard $1 \times$ rate.

\section{Effects of Mixed Spray Programs}

The fast increase in disease previously reported for the short-term schedules of fungicidal sprays was avoided by using mixed spray programs, in which applications of the $0.5 \times$ or $1 \times$ fungicidal rate followed early sprays of $0.25 \times$ mancozeb. The mixed schedule I had four sprays of the $0.25 \times$ rate plus two sprays of the $1 \times$. The mixed schedule II consisted of four sprays of the $0.25 \times$ rate plus four sprays of the $0.5 \times$ rate. In the mixed schedule III, six sprays of the $0.25 \times$ rate were followed by three sprays of the $0.5 \times$ rate of mancozeb. The mixed spray programs were initiated on day 84 in 1996 and on day 68 in 1997. The spray intervals were twice-weekly for the $0.25 \times$, weekly for the $0.5 \times$, and $10-12$ days for the $1 \times$ rate of mancozeb.

In 1996, the mixed schedules II and III resulted in control of HLS as good as that provided by 12 sprays of $0.25 \times$ mancozeb alone (Table 4.6 and Fig. 4.10 ). In the mixed 
program I, the early sprays of the $0.25 \times$ rate reduced $y_{0}$ significantly $(P=0.05)$, but the following applications of the $1 \times$ rate, at longer spray intervals, were unable to keep the epidemic rate slow. Therefore, the mixed program I had higher $y_{f}$ and AUDPC than the $0.25 \times$ rate alone. Compared to the standard $1 \times$ treatment, however, the mixed program I reduced the final amount of disease and the AUDPC significantly $(P=0.05)$ (Table 4.6). Since the epidemic rates did not differ for rye sprayed with the standard $1 \times$ program and the mixed schedule I, the better control of HLS provided by the latter program was credited to the reduction of $y_{0}$ by the early sprays of the $0.25 \times$ fungicidal rate.

Table 4.6. Initial $\left(y_{0}\right)$ and final $\left(y_{f}\right)$ disease proportions, estimated epidemic rates $\left(r_{G N L}\right)$, and areas under the disease progress curves (AUDPC) associated with epidemics of helminthosporium leaf spot on rye plants subjected to mixed-rate spray schedules of the fungicide mancozeb in 1996. The proportions of disease severity were assessed on flag, $\mathrm{f}-1, \mathrm{f}-2$, and $\mathrm{f}-3$ leaves

\begin{tabular}{lcccc}
\hline & \multicolumn{4}{c}{ Epidemic parameters } \\
\cline { 2 - 5 } Spray program $^{\mathrm{x}}$ & $\boldsymbol{y}_{0}$ & $\boldsymbol{y}_{f}$ & $\boldsymbol{r}_{G N L}{ }^{\mathrm{y}}$ & AUDPC \\
\hline $0.25 \times$ & $0.001 \mathrm{a}^{\mathrm{z}}$ & $0.06 \mathrm{a}$ & $0.02 \mathrm{a}$ & $0.84 \mathrm{a}$ \\
Mixed III & $0.001 \mathrm{a}$ & $0.07 \mathrm{a}$ & $0.021 \mathrm{a}$ & $1.01 \mathrm{ab}$ \\
Mixed II & $0.001 \mathrm{a}$ & $0.08 \mathrm{a}$ & $0.025 \mathrm{a}$ & $0.98 \mathrm{ab}$ \\
Mixed I & $0.001 \mathrm{a}$ & $0.12 \mathrm{~b}$ & $0.029 \mathrm{~b}$ & $1.33 \mathrm{~b}$ \\
Standard 1x & $0.004 \mathrm{~b}$ & $0.18 \mathrm{c}$ & $0.032 \mathrm{~b}$ & $2.32 \mathrm{c}$ \\
Nonsprayed & $0.005 \mathrm{~b}$ & $0.36 \mathrm{~d}$ & $0.045 \mathrm{c}$ & $5.03 \mathrm{~d}$ \\
\hline$P>F$ & 0.001 & 0.0001 & 0.0001 & 0.0001 \\
\hline
\end{tabular}

${ }^{x}$ Mixed-rate programs began 84 days after planting and consisted of four sprays of the $0.25 \times$ rate plus two sprays of the $1 \times$ rate (Mixed $\mathrm{I}$ ), four sprays of the $0.25 \times$ rate plus four sprays of the $0.5 \times$ rate (Mixed II), or six sprays of the $0.25 \times$ rate plus three sprays of the $0.5 \times$ rate (Mixed III). Spray intervals were twice-weekly for the $0.25 \times$ rate, weekly for the $0.5 \times$, and $10-12$ days for the $1 \times$ rate of mancozeb. The $0.25 \times$ treatment was applied 12 times from day 84 . The standard schedule consisted of three sprays of $1 \times$ mancozeb on days 91,103 , and 114 .

${ }^{y}$ Epidemic rates were estimated by nonlinear regression of disease proportions against time using the Gompertz model.

z Treatment means within columns followed by the same letter do not differ significantly $(P>$ 0.05 ) by Duncan's Multiple Range Test, using the variability pooled among replicates. Even though rate of fungicide is a quantitative factor, the individual rates were applied at different intervals and therefore the treatments were considered unique. 
Figure 4.10. Disease progress curves (A) and epidemic waves (B) for helminthosporium leaf spot on rye plants subjected to mixed-rate schedules of fungicidal sprays in 1996. Mixed-rate programs began 84 days after planting and consisted of four sprays of the $0.25 \times$ rate plus two sprays of the $1 \times$ rate (Mixed I), four sprays of the $0.25 \times$ rate plus four sprays of the $0.5 \times$ rate (Mixed II), or six sprays of the $0.25 \times$ rate plus three sprays of the $0.5 \times$ rate (Mixed III). Spray intervals were twice-weekly for the $0.25 \times$ rate, weekly for the $0.5 \times$ rate, and 10-12 days for the $1 \times$ rate of mancozeb. The $0.25 \times$ treatment was applied 12 times from day 84 . The standard schedule had three sprays of $1 \times$ mancozeb on days 91 (growth stage 32 in the decimal code scale; 7 in the Feekes scale), 103, and 114. 


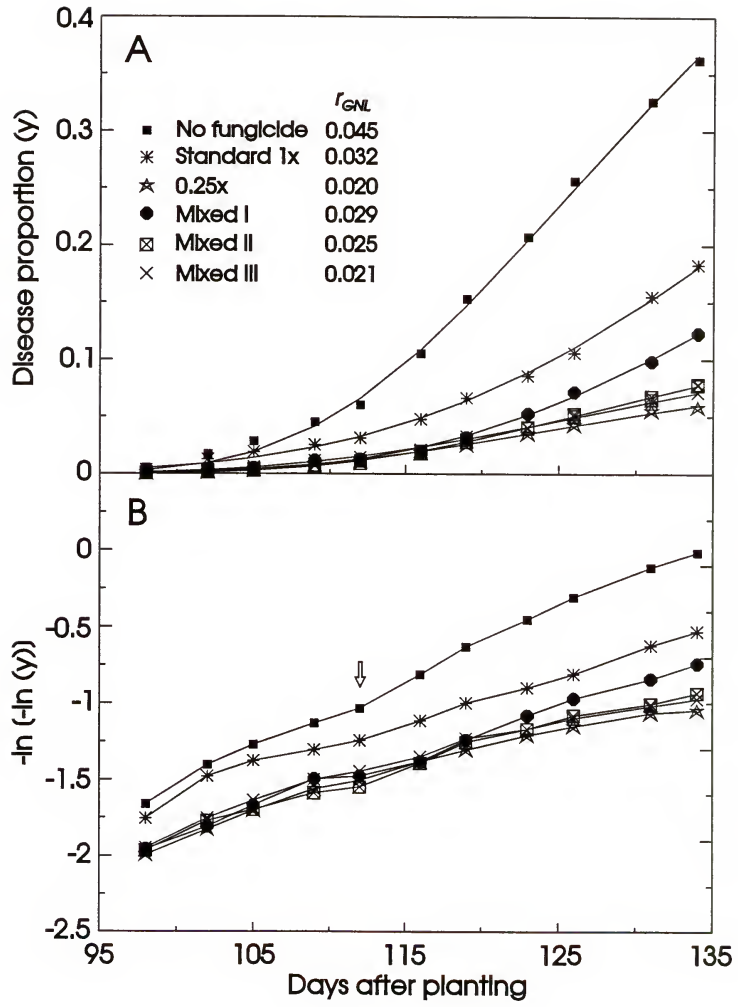


The results obtained in 1997 (Table 4.7 and Fig. 4.11) were similar to those reported for the 1996 experiment. The $y_{0}, r_{G N L}, y_{\beta}$ and AUDPC of the mixed schedules II and II equaled $(P=0.05)$ those of the $0.25 \times$ treatment alone. The mixed schedule I had an AUDPC (2.17) higher than the $0.25 \times$ treatment (1.38) but lower than the standard spray program (3.95).

Table 4.7. Initial $\left(y_{0}\right)$ and final $\left(y_{f}\right)$ disease proportions, estimated epidemic rates $\left(r_{G N L}\right)$, and areas under the disease progress curves (AUDPC) associated with epidemics of helminthosporium leaf spot on rye plants subjected to mixed-rate spray schedules of the fungicide mancozeb in 1997. The proportions of disease severity were assessed on flag, $f-1, f-2$, and $f-3$ leaves

\section{Epidemic parameters}

\begin{tabular}{lllll}
\cline { 2 - 6 } Spray program $^{x}$ & \multicolumn{1}{c}{$\boldsymbol{y}_{0}$} & $\boldsymbol{y}_{f}$ & $\boldsymbol{r}_{G N L}{ }^{\mathrm{y}}$ & AUDPC \\
\hline $0.25 \times$ & $0.003 \mathrm{a}^{\mathrm{z}}$ & $0.12 \mathrm{a}$ & $0.027 \mathrm{a}$ & $1.38 \mathrm{a}$ \\
Mixed III & $0.005 \mathrm{ab}$ & $0.145 \mathrm{a}$ & $0.032 \mathrm{ab}$ & $1.54 \mathrm{ab}$ \\
Mixed II & $0.003 \mathrm{a}$ & $0.16 \mathrm{a}$ & $0.031 \mathrm{ab}$ & $1.86 \mathrm{ab}$ \\
Mixed I & $0.003 \mathrm{a}$ & $0.18 \mathrm{a}$ & $0.033 \mathrm{~b}$ & $2.17 \mathrm{~b}$ \\
Standard 1x & $0.01 \mathrm{~b}$ & $0.295 \mathrm{~b}$ & $0.039 \mathrm{c}$ & $3.95 \mathrm{c}$ \\
Nonsprayed & $0.01 \mathrm{~b}$ & $0.58 \mathrm{c}$ & $0.064 \mathrm{~d}$ & $8.18 \mathrm{~d}$ \\
\hline$P>F$ & 0.015 & 0.0001 & 0.0001 & 0.0001 \\
\hline
\end{tabular}

${ }^{x}$ Mixed-rate programs began 68 days after planting and consisted of four sprays of the $0.25 \times$ rate plus two sprays of the $1 \times$ rate (Mixed I), four sprays of the $0.25 \times$ rate plus four sprays of the $0.5 \times$ rate (Mixed II), or six sprays of the $0.25 \times$ rate plus three sprays of the $0.5 \times$ rate (Mixed III). Spray intervals were twice-weekly for the $0.25 \times$ rate, weekly for the $0.5 \times$, and $10-12$ days for the $1 \times$ rate of mancozeb. The $0.25 \times$ treatment was applied 12 times from day 68 . The standard schedule consisted of three sprays of $1 \times$ mancozeb on days 82,93 , and 105 .

${ }^{y}$ Epidemic rates were estimated by nonlinear regression of disease proportions against time using the Gompertz model.

${ }^{2}$ Treatment means within columns followed by the same letter do not differ significantly ( $P>$ 0.05 ) by Duncan's Multiple Range Test, using the variability pooled among replicates. Even though rate of fungicide is a quantitative factor, the individual rates were applied at different intervals and therefore the treatments were considered unique.

\section{Relationship between AUDPC and Yield}

Helminthosporium leaf spot reduced the rye yield significantly in both the 1996 and 1997 experiments. The relationship of AUDPC to kernel yield (Fig. 4.12) was 
Figure 4.11. Disease progress curves (A) and epidemic waves (B) for helminthosporium leaf spot on rye plants subjected to mixed-rate schedules of fungicidal sprays in 1997. Mixed-rate programs began 68 days after planting and consisted of four sprays of the $0.25 \times$ rate plus two sprays of the $1 \times$ rate (Mixed I), four sprays of the $0.25 \times$ rate plus four sprays of the $0.5 \times$ rate (Mixed $I$ ), or six sprays of the $0.25 \times$ rate plus three sprays of the $0.5 \times$ rate (Mixed III). Spray intervals were twice-weekly for the $0.25 \times$ rate, weekly for the $0.5 \times$ rate, and 10-12 days for the $1 \times$ rate of mancozeb. The $0.25 \times$ treatment was applied 12 times from day 68 . The standard schedule had three sprays of $1 \times$ mancozeb on days 82 (growth stage 32 in the decimal code scale; 7 in the Feekes scale), 93, and 105 . 


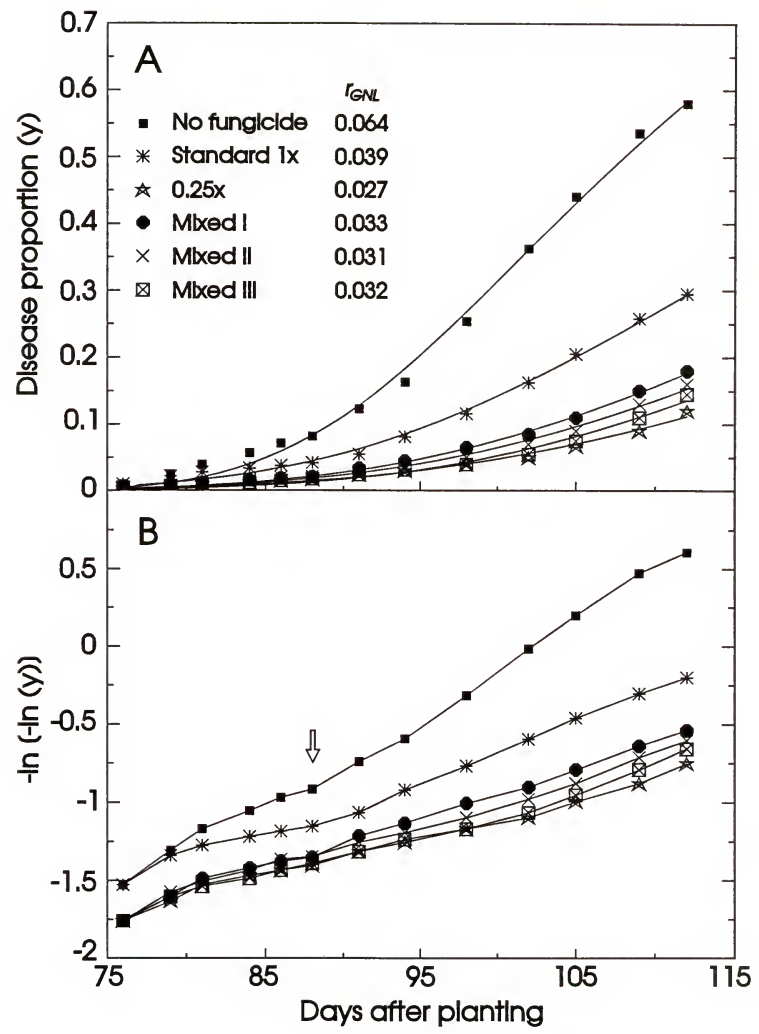


represented by the linear equations $Y_{1996}=3650-241.8 X, R^{2}=0.97$, and $Y_{1997}=2545$ 82.7X, $R^{2}=0.93$, where $Y$ is kernel yield in $\mathrm{kg} \mathrm{ha}^{-1}$ and $X$ is AUDPC. Due to a better stand of plants in the field plots and to an overall lower disease severity, the yield averaged over all treatments was higher in $1996\left(3120 \mathrm{~kg} \mathrm{ha}^{-1}\right)$ than in $1997(2283 \mathrm{~kg}$ $\mathrm{ha}^{-1}$ ). Although a lower range of AUDPC ( 0.84 to 5.03), the range in kernel yield (highest - lowest yield) associated with HLS was greater in $1996\left(1015 \mathrm{~kg} \mathrm{ha}^{-1}\right)(P=$ $0.0003)$ than in $1997\left(620 \mathrm{~kg} \mathrm{ha}^{-1}\right)(P=0.01)$.

Table 4.8. Kernel yield of rye plants subjected to spray programs of the fungicide mancozeb to control helminthosporium leaf spot in 1996 and 1997

\begin{tabular}{llc}
\hline & \multicolumn{2}{c}{ Kernel yield $\left(\mathbf{k g ~ h a}^{-1}\right)$} \\
\cline { 2 - 3 } Spray program & $\mathbf{1 9 9 6}$ & $\mathbf{1 9 9 7}$ \\
\hline $0.25 \times$ & $3427 \mathrm{a}^{\mathbf{2}}$ & $2417 \mathrm{a}$ \\
Mixed III & $3409 \mathrm{a}$ & $2504 \mathrm{a}$ \\
Mixed II & $3399 \mathrm{a}$ & $2369 \mathrm{a}$ \\
Mixed I & $3350 \mathrm{a}$ & $2381 \mathrm{a}$ \\
$0.5 \times$ & $3272 \mathrm{ab}$ & $2357 \mathrm{a}$ \\
Standard 1x & $3147 \mathrm{ab}$ & $2208 \mathrm{ab}$ \\
Short II & $3145 \mathrm{ab}$ & - \\
$0.25 \times$-delay & $2917 \mathrm{bc}$ & $2144 \mathrm{bc}$ \\
Short I & $2722 \mathrm{~cd}$ & - \\
No fungicide & $2412 \quad \mathrm{~d}$ & $1884 \mathrm{c}$ \\
\hline$P>F$ & 0.0003 & 0.01 \\
\hline
\end{tabular}

${ }^{z}$ Treatment means within columns followed by the same letter do not differ significantly $(P>$ 0.05 ) by Duncan's Multiple Range Test. Even though rate of fungicide is a quantitative factor, the individual rates were applied at different intervals and therefore the treatments were considered unique.

Most fungicidal spray programs increased the kernel yield of sprayed plants significantly $(P=0.05)$ (Table 4.8$)$. Delay $(0.25 \times$-delay) or early discontinuation (Short I) of fungicidal sprays, however, resulted in low kernel yield. The differences in yield between the reduced- or mixed-rate programs and the standard fungicidal treatments were not significant. 


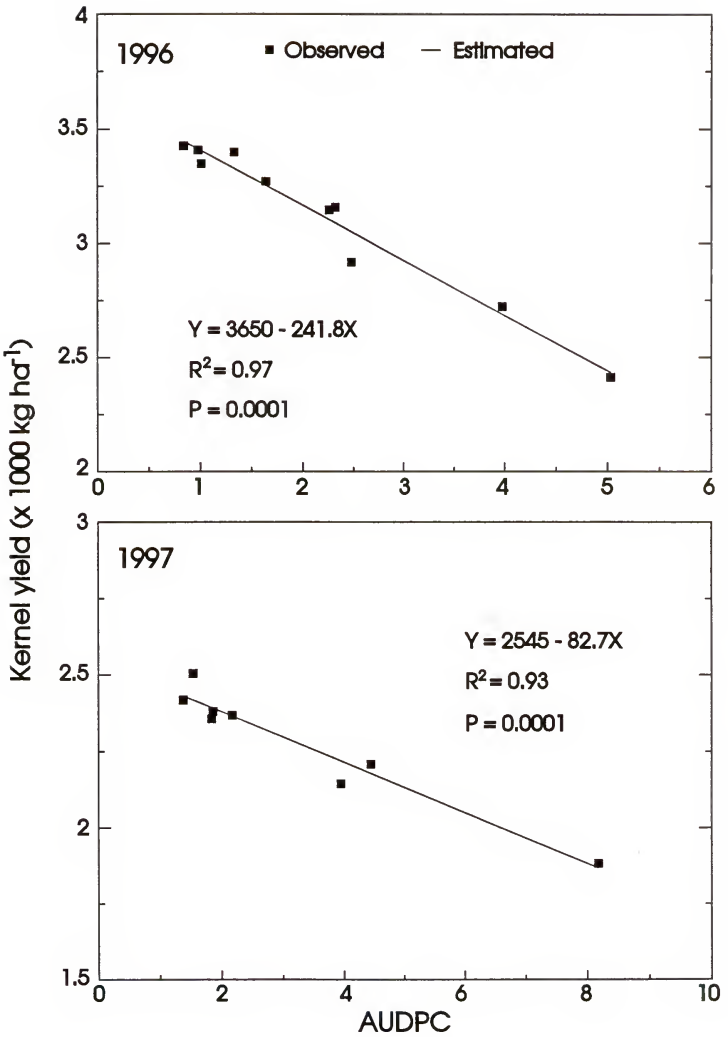

Figure 4.12. Relationship of area under the disease-progress curve (AUDPC) to kernel yield associated with epidemics of helminthosporium leaf spot on rye in 1996 and 1997. 


\section{$\underline{\text { Discussion }}$}

Because little information is available on the control of rye diseases, the Florida disease-control program for wheat (Kucharek and Luke, 1989; Kucharek, 1994c) was adapted to rye and used as the standard spray program for control of HLS. This program is based on three applications of the fungicide mancozeb initiated at the plant growth stage 32 (or 7 in the Feekes scale) and repeated at 10- to 14-day intervals. Although applications of fungicide according to the standard program had no effect on the amount of initial disease $\left(y_{0}\right)$, the final $\left(y_{f}\right)$ and total disease (AUDPC) were significantly lower on sprayed than on nonsprayed plants. Plants treated with fungicide also yielded more than nonsprayed plants.

Modification of the standard (1x) spray schedule to allow for early and shortinterval applications of lower rates $(0.25 \times$ and $0.5 \times)$ of fungicide improved the control of HLS significantly. In both the 1996 and 1997 growing seasons, plants sprayed with the reduced fungicidal rates had lower initial $\left(y_{0}\right)$, final $\left(y_{f}\right)$, and total disease (AUDPC) than plants subjected to the standard treatment. Reduction of $y_{0}$ resulted in a better disease control (lower $y_{f}$ and AUDPC) even when the epidemic rate of HLS did not differ between the low-rate $(0.5 \times$ in 1996$)$ and the full-rate treatments.

The various fungicidal spray programs tested for the control of HLS had no effect on epidemic onset. Bipolaris sorokiniana survives on plant debris left in the field, as well as in volunteer plants (Kucharek, 1994b). In both 1996 and 1997 growing seasons, rye was grown in fields previously planted to the same crop. The presence of inoculum from the previous crop, in addition to using seeds infected with the HLS fungus, provided an early and even distribution of the disease among plants. Consequently, the disease 
was already established in plants when fungicidal treatments were initiated. Berger (1977) pointed out the benefits of sanitation and of the use of disease-free seeds on the management of monocyclic and polycyclic diseases. Kucharek (1994b) recommended crop rotation and use of disease-free seeds to control HLS in rye. Therefore, improved disease control could be obtained if fungicidal spray programs were preceded by crop rotation and the use of disease-free or treated seeds.

In the experiments on management of HLS, a lower-rate spray program $(0.25 \times-$ delay) was delayed in 7 days (1996) or 14 days (1997) to coincide the first spray application with the initiation of the standard $1 \times$ program at the growth stage 32 . The results on $y_{0}, y_{f}$ AUDPC, and $r_{G N L}$ were not different between $0.25 \times$-delay and the standard treatment, that is, both spray programs provided the same disease control. Compared to earlier sprays of the $0.25 \times$ rate, however, the delay treatment had faster $r_{G N L}$ and higher $y_{0}, y_{\beta}$ and AUDPC. Thus, simple replacement of the standard treatment by twice-weekly sprays of the lower rate ( $0.25 \times$-delay) did not improve the control of HLS. To do so, it was also necessary to reduce the initial epidemic wave, which was attained by sprays of fungicide initiated earlier than the growth stage (32) recommended for the standard spray program.

Fry (1977 and 1982) noted that the effects exerted by fungicides on epidemics depend on how often they are used. If the chemical is applied only once or just a few times at the beginning of the epidemic, the major effect is to reduce the initial disease. If the treatment is repeated during the season, however, both the amount of initial disease and the epidemic rate are reduced. In 1996, applications of the $0.25 \times$ rate for only 2 weeks reduced the initial epidemic wave only partially, and these sprays were not able to 
control HLS. After fungicidal sprays have ceased, the disease progressed at an epidemic rate faster than that of nonsprayed plants. Discontinuation of the spray program after 3 weeks reduced the epidemic wave significantly but again resulted in a fast epidemic rate. Berger (1975a, 1977, and 1988) had already warned about this problem. It seemed clear that the reduction of initial disease waves provided by early sprays of fungicide should be complemented with subsequent applications to keep the epidemic rate slow and the disease under control. To account for this problem, mixed-rate schedules of fungicidal sprays were evaluated for the control of HLS. In both 1996 and 1997 experiments, the mixed-rate schedules provided control of disease comparable to that obtained by seasonlong sprays of the $0.25 \times$ rate. Therefore, the mixed-rate approach may be a technically reliable and economically viable strategy to control diseases.

Although differences in yield were significant between sprayed and nonsprayed plants, little variation in kernel yield was observed among the various fungicidal schedules. Rye plants have only few, short, narrow leaves but long stems. The reduction in leaf area caused by HLS might have been compensated for by the photosynthetic activity of the stem tissue. Overall, delay or early discontinuation of fungicidal sprays resulted in low kernel yield. 


\section{CHAPTER V \\ MANAGEMENT OF EPIDEMICS OF RUST AND LEAF SPOTS ON PEANUT}

\section{Introduction}

Early and late leaf spots caused by Cercospora arachidicola S. Hori and Cercosporidium personatum (Berk. \& M. A. Curtis) Deighton, respectively, are two of the most important diseases of peanut throughout the world (Porter et al., 1984). Both diseases cause severe plant defoliation which results in significant yield reduction of the peanut crop (Backman and Crawford, 1984; Gorbet et al., 1982; Kucharek, 1979; Shokes and Gorbet, 1990; Smith and Littrell, 1980). In severely diseased fields, yield reductions of $1,100 \mathrm{~kg} \mathrm{ha}^{-1}$ or higher have been reported (Jacobi and Backamn, 1991). In Florida, annual losses in pod yield attributable to peanut leaf spots vary from 5 to $20 \%$ (Kucharek et al., 1991). These losses can be higher if the management of peanut leaf spots is not adequate (Smith and Littrell, 1980).

Early and late leaf spot are differentially distributed among the peanut-growing regions of the U.S. Early leaf spot is more prevalent in Virginia, North Carolina, Oklahoma, and New Mexico, whereas late leaf spot predominates in Florida. In Texas, Alabama, Georgia, and South Carolina both diseases are equally prevalent (Nutter and Shokes, 1995). The predominance of one disease over the other, however, can shift in any given year or over years in response to changes in climate (Brenneman and Culbreath, 1994; Nutter and Shokes, 1995). 
Peanut leaf spots are primarily controlled by protectant fungicides applied on a calendar schedule. The current recommendation is to initiate fungicide applications 30 to 40 days after planting and continue on a 10- to 14-day interval until 14 days prior to digging (Davis et al., 1993; Jacobi et al., 1995; Kucharek, 1979; Kucharek and Shokes, 1995; Linvill and Drye, 1995; Smith and Littrell, 1980). On average, 7-9 fungicidal sprays are made during the season (Brenneman et al., 1990; Brenneman and Murphy, 1991; Labrinos and Nutter, 1993) and chlorothalonil is used $90 \%$ of the time (Labrinos and Nutter, 1993). Recently, systemic fungicides such as propiconazole and tebuconazole have also been recommended for the control of peanut leaf spots (Brenneman and Murphy, 1991; Brenneman and Culbreath, 1994; Kucharek and Shokes, 1995; Labrinos and Nutter, 1993),

Kucharek et al. (1991) presented a comprehensive review on the control of peanut leaf spots by fungicides. Some variables that enhance the control of peanut leaf spot are early spray initiation, delayed spray termination, short intervals between sprays, use of more efficacious fungicides early in the spray program, and spray nozzle orientation that concentrates spray along the center of rows. Spray pressure and volume, sprayer speed, and nozzle type and size did not influence control of peanut leaf spot (Riabov, 1978; Kucharek et al., 1986).

Additional control measures for peanut leaf spots are elimination of volunteer plants, deep plowing of the crop refuse, crop rotation, and cultivar resistance (Kucharek 1979, Kucharek and Shokes, 1995; Nutter and Shokes, 1995; Porter et al., 1984). Kucharek (1975) reported that crop rotation reduced the early-season incidence of peanut leaf spots by $88-93 \%$. Cultivars with partial resistance have been developed but the levels of resistance are still insufficient to preclude the need for fungicidal applications 
(Gorbet et al., 1986; Johnson and Beute, 1986; Shew et al., 1995; Watson, 1987). The rate-reducing resistance of some peanut cultivars has allowed sprays of fungicide at longer intervals, thus reducing the total fungicide required for disease control (Culbreath et al., 1992; Jacobi and Backman, 1995; Kucharek et al., 1991; Kucharek and Shokes, 1995; Maytac and Bailey, 1988).

Peanut rust, caused by Puccinia arachidis Speg., is another important fungal disease of the peanut crop (Porter et al., 1984). In Florida, this disease occurs sporadically and usually no earlier than August (Kucharek, 1979). However, if rust occurs early in the season, the peanut yield can be reduced $40-70 \%$ Nutter and Shokes, 1995). Peanut rust epidemics have been reported to increase faster than epidemics caused by leaf spotting fungi (Nutter and Shokes, 1995).

Control of peanut rust has been restricted to fungicides since cultivars fully resistant to rust are not available (Nutter and Shokes, 1995). Fungicidal applications to control rust must be initiated as soon as the first pustules are detected in the field, (Kucharek, 1979) and repeated at 7- to 10-day intervals (Nutter and Shokes, 1995). The fungicides chlorothalonil, mancozeb plus sulfur, and tebuconazole have been recommended to control peanut rust (Kucharek, 1979; Kucharek and Shokes, 1995; Nutter and Shokes, 1995).

Because the control of both leaf spots and rust relies largely on chemical control, alternative approaches have been studied to improve fungicidal spray programs. Fixed interval or calendar-based spray programs may schedule fungicidal applications during periods when environmental conditions are unfavorable for infection of peanut plants by the rust or the leaf-spotting fungi. Conversely, during periods favorable for disease establishment, fixed schedules may recommend fungicide applications too infrequently 
(Jacobi and Backman, 1995). For this reason, several weather-based schedules have been developed and tested to time fungicidal sprays on peanuts (Bailey et al., 1994; Brenneman and Culbreath, 1994; Davis et al., 1993; Jacobi and Backman, 1995; Jacobi et al., 1995; Linvill and Drye, 1995; Wu et al., 1996). These advisory programs are based on rainfall amounts, or the combination of relative humidity and temperature, that favors the occurrence of disease. AU-Pnuts, an advisory system developed at Auburn University (Jacobi and Backman, 1995; Jacobi et al., 1995), has been tested and proven reliable for peanut-growing areas in Florida (Kucharek and Shokes, 1995).

Most disease advisory systems, however, are not predictive, so that by the time disease-favorable weather conditions trigger a spray recommendation, disease progress is already under way (Wilks and Schen, 1991). Additionally, the logistics of applying fungicides may further delay action for several more days (Bailey et al., 1994). The applicability of disease advisory systems may also be affected by simultaneous occurrence of other diseases. For instance, no information is available concerning the applicability of the weather-based schedules developed for leaf spots on the control of peanut rust. Finally, neither the calendar- nor the weather-based fungicide programs protect the new plant tissue that develops between sprays.

To improve the control of peanut leaf spots and peanut rust, alternative spray programs, based on applications of low fungicidal rates at short spray intervals, were evaluated in this research. The main objectives of such reduced-rate spray programs were to suppress and to delay the initial waves of disease and thus reduce the total epidemic. 


\section{Material and Methods}

A total of seven experiments on the peanut-rust and peanut-leaf spot pathosystems were developed in the greenhouse (1) and in the field (6) during the cropping seasons of 1995,1996 , and 1997. In the first experiment developed in 1995, spray programs based on applications of reduced rates of fungicide at short time intervals were compared to the AU-Pnuts advisory system and to a standard, calendar-based spray program initiated on either of two times after planting. In 1996, all the reduced-rate treatments and the standard spray schedule were begun at two levels of initial disease to evaluate the effect of action thresholds on the management of peanut leaf spots with fungicides. Because crop rotation is highly recommended to reduce the initial inoculum of both $C$. arachidicola and C. personatum (Kucharek, 1975; Kucharek and Shokes, 1995; Nutter and Shokes, 1995), the 1996 experiment was conducted with monoculture of peanuts and with crop rotation. Also in 1996, an additional experiment was developed to evaluate reduced-rate spray programs for Southern Runner, a peanut cultivar with partial resistance to late leaf spot (Gorbet et al., 1986; Watson, 1987). In 1997, emphasis was given to the study of mixed spray programs, which included early-season sprays of low fungicidal rates $(0.25 \times$ and $0.5 \times)$ followed by applications of the standard $1 \times$ rate. Results on disease intensity and pod yield of peanuts obtained in the various experiments conducted over 3 years were used to study some relationships between disease and yield.

\section{Disease Efficiency of $P$. arachidis and C. arachidicola}

The objective of this experiment was to evaluate the control of rust and early leaf spot of peanuts provided by applications of a lower rate $(0.25 \times)$ of fungicide at a shorter time interval than the standard spray program $(1 \times)$. This experiment was conducted 
under controlled inoculations of the rust and leaf spot fungi. The fungicidal treatments were evaluated based on the reduction they provided on the disease efficiency of $P$. arachidis and $C$. arachidicola.

Seeds of Florunner peanuts were pre-germinated in the laboratory and sown in clay pots $(20 \mathrm{~cm}$ diameter $\times 21 \mathrm{~cm}$ high) containing the growing medium, Metro-Mix 300, on 18 August 1995. Twenty days later, the seedlings were thinned to three plants per pot. For each treatment, four pots were used as replicates. The plants were fertilized weekly by adding $5 \mathrm{~g}$ of a $10-10-10(\mathrm{~N}-\mathrm{P}-\mathrm{K})$ fertilizer to the growing medium. All plants were grown outdoors and exposed to ambient conditions. Sprays of the fungicide chlorothalonil were initiated 35 days after planting and repeated twice-weekly $(0.25 \times$ rate) or biweekly ( $1 \times$ rate). At the time of fungicide application, four unfolded leaves in each plant were marked with a permanent color marker for later disease assessment. Plants not treated were misted with water twice a week. Fungicidal treatments were applied onto plants at least 2 hours prior to inoculations, which were begun 35 days after planting and were repeated every other day. Inoculum suspensions for C. arachidicola were prepared as diseased peanut leaves were washed in distilled water plus Tween- 20 ( $0.05 \mathrm{ml}$ per $100 \mathrm{ml}$ of water) and the inoculum density adjusted to $2 \times 10^{4}$ conidia $\mathrm{ml}^{-1}$. Uredospores of $P$. arachidis were then added to the same spore suspension for a final density of $4 \times 10^{4}$ uredospores $\mathrm{ml}^{-1}$. The volume of inoculum suspension applied to each set of four pots ( 12 plants) ranged from $50 \mathrm{ml}$ to $80 \mathrm{ml}$ depending on plant age and size. Inoculations were always carried out between 19:00 and 20:00 hours. After every inoculation, ten leaflets were carefully collected, slightly dried, and assessed for the number of uredospores or conidia in $1 \mathrm{~cm}^{2}$ on the adaxial leaf surface. After inoculation, 
plants were covered with plastic bags for 16 hours and moved into the greenhouse for incubation. The temperature inside the greenhouse ranged from $20^{\circ} \mathrm{C}$ to $35^{\circ} \mathrm{C}$ during this experiment. Fourteen days after the inoculation, all marked leaves were collected, examined for the presence of pustules or leaf spots, and measured by means of a portable leaf area meter. The disease efficiency (\%) for each pathogen was then calculated based on the ratio of number of lesions per $\mathrm{cm}^{2}$ of leaf $\times 100$ to the number of propagules inoculated per $\mathrm{cm}^{2}$ of leaf tissue.

\section{Experiments in the Field}

Field experiments on peanuts were conducted during the 1995, 1996, and 1997 cropping seasons in a field located southwest of Lake Alice, on the University of Florida campus, in Gainesville. The peanut cultivar Florunner, susceptible to both peanut rust and peanut leaf spots (Norden et al., 1979), was planted in all three seasons. In 1996, an additional experiment was planted to Southern Runner, a cultivar with moderate resistance to leaf spots (Gorbet et al., 1986; Watson, 1987). All experiments followed previous crops of oat or rye. The top soil layer was plowed to $0.20 \mathrm{~m}$ depth, $250 \mathrm{~kg} \mathrm{ha}^{-1}$ of a hand-mixed 0-20-35 (N-P-K) fertilizer was spread, and the soil disked again to a 0.15 $m$ depth. The last two operations were carried out 2 days before planting. Before sowing, peanut seeds were inoculated with Bradyrhizobium bacteria (cowpea group) at the rate of $2 \mathrm{~g}$ of substrate per kilogram of seed. Plot sizes ranged from $1.82 \mathrm{~m} \times 4 \mathrm{~m}$ (1997) to $2.73 \mathrm{~m} \times 3 \mathrm{~m}$ (1996) to $3.64 \mathrm{~m} \times 4 \mathrm{~m}$ (1995). In all seasons, the space between rows and between plots was $0.91 \mathrm{~m}$. Plots were arranged according to a randomized complete block design with five replicates. Peanuts were sown manually 5-7 $\mathrm{cm}$ deep at the rate of 17 seeds per linear meter. A supplement of $650 \mathrm{~kg} \mathrm{ha}^{-1}$ of gypsum ( $\geq 98 \%$ 
$\mathrm{CaSO}_{4}$ ) was spread in an 18 -inch band along rows, 40 days after planting. Supplemental irrigation was applied as needed to maintain optimum plant growth. Weeds were controlled either manually or chemically, and insecticides were applied as needed to control insects. $\mathrm{A} \mathrm{CO}_{2}$ backpack sprayer connected to a 2-meter spray boom with six Conejet (hollow cone spray tip) nozzles was used to deliver the fungicide. The spray pressure (2.8 to $\left.3.5 \mathrm{~kg} \mathrm{~cm}^{-2}\right)$ and the operator's speed were adjusted to deliver about 2361 ha $^{-1}$ (Kucharek et al., 1991; Kucharek and Shokes, 1995). Disease severity was assessed twice a week only from the center rows of each plot. In the early stages of the epidemics, diseased leaf area was estimated by determining the incidence of diseased leaflets per plant which were then graded according to a standard leafspot diagram (Shokes et al., 1987). In later stages, the disease severity was estimated by visual evaluation of plants at three points within the center rows and at three canopy layers (Plaut and Berger, 1980) within each point. The average of these disease assessments was corrected for defoliation according to the formula $y_{t}=\left[(1-d) y_{v}\right]+d$, where $y_{t}$ and $y_{v}$ are the total and visible disease severities, respectively, and $d$ is the defoliation determined by the proportion of abscised leaflets (Plaut and Berger, 1980; Shokes et al., 1987). At maturity, all plants in the center rows were dug manually, dried in the field or in hot-air dryers, and the pods removed by hand. Pods were additionally dried to $10 \%$ moisture and then weighed to determine pod yields.

\section{Effect of low rates of fungicide on control of disease and yield in 1995}

This experiment was planted on 14 May 1995 in a field that had not been planted to peanuts the previous 2 years. Cercospora arachidicola was inoculated 42 days after planting by applying a spore suspension containing at least $2 \times 10^{4}$ conidia $\mathrm{ml}^{-1}$ to two plants in each plot. The inoculum suspension was obtained by washing diseased leaves 
collected from other fields and incubated in a dew chamber for 3 days. Puccinia arachidis was inoculated 21 days later at a minimum density of $4 \times 10^{4}$ uredospores $\mathrm{ml}^{-1}$. The uredospores were collected from volunteer plants found 200 yards northwest of the field plots. Both inoculations were made in late afternoon and inoculated plants remained covered by plastic bags for about 14 hours.

Several fungicidal spray programs were tested in this experiment. The standard treatment with $1 \times$ the label rate $\left(1.25 \mathrm{~kg}\right.$ a.i. ha $\left.{ }^{-1}\right)$ of chlorothalonil (Terranil $90 \mathrm{DF}$, Riverside-Terra Co., Sioux City, IA), applied at 2-week intervals, was compared to weekly sprays of the $0.5 \times$ rate and twice-weekly sprays of the $0.25 \times$ or $0.1 \times$ rates. Fungicidal treatments were initiated 42 days after planting so that the $1 \times$ rate was applied four, the $0.5 \times$ rate seven, and the $0.25 \times$ and $0.1 \times$ rates fourteen times. The standard treatment was also compared to a delay treatment (1x-delay) initiated 7 days later (day 49) and also to another treatment applied based on a forecast system (AU-Pnuts) (Jacobi et al., 1995). The latter spray program was initially sprayed on day 42 and repeated after every three rain events, but not within 10 days of the previous spray. A rain event was a day with rainfall or irrigation $\geq 2.5 \mathrm{~mm}$. The $1 \times$-delay treatment was sprayed four and the AU-Pnuts five times during the season. Plants were harvested on 9 September 95. Management of epidemics with low rates of fungicide with monoculture of peanuts

This experiment was established on 18 May 1996 in an area planted to peanuts the previous year. Peanut plants were not inoculated with the leaf spot fungi because natural inoculum of both $C$. arachidicola and $C$. personatum were available on diseased volunteer plants that developed during the spring months. Diseased leaves collected from the volunteer plants showed conidia of both leaf spot fungi when placed in dew chamber 
for 3 days. A spore suspension containing about $4 \times 10^{4}$ uredospores of $P$. arachidis per $\mathrm{ml}$ was applied to plants in the border rows of each plot 45 days after planting. To vary the amount of leaf spots at the beginning of fungicidal treatments, the $0.25 \times-, 0.5 \times-$, and $1 \times$-spray programs were initiated at 38 and 45 days after planting. The $0.25 \times$ rate was applied 17 times (twice-weekly), the $0.5 \times$ rate nine times (weekly), and the $1 \times$ rate five times (biweekly) during the season. Additional spray programs tested in this experiment included $i$, six sprays of the $1 \times$ rate according to the AU-Pnuts advisory system, $i i$, four sprays of the systemic fungicide tebuconazole (Folicur 3.6 F, Bayer Corporation, Kansas City, MO), at $206 \mathrm{~g}$ a.i. ha ${ }^{-1}$, applied on days $45,59,74$, and 88 , and iii, eight sprays (twice-weekly) of the $0.25 \times$ rate, from day 38 to 63 , followed by two applications (biweekly) of the $1 \times$ rate on days 75 and 89 .

\section{Management of epidemics with low rates of fungicide after crop rotation or fallow}

In this trial, the peanut plants were grown in an area not planted to the same crop the last 3 years. Also, no volunteer peanut plants were detected in the same area during the months preceding the planting. Although the crop rotation experiment was located only a few meters west of the area having a monoculture of peanuts, alloinfection was regarded as negligible since the predominant winds blow from west to east. Peanut plants were inoculated with $P$. arachidis 45 days after planting as described above. The same fungicidal spray programs tested in the monoculture experiment were included in the crop rotation trial, except that all treatments were delayed one week due to late disease occurrence. 
Management of epidemics with low rates of fungicide on a peanut variety with partial resistance to disease

The experiment with the partially resistant variety Southern Runner was planted on 18 May 1996 in the area of monoculture of peanuts. Therefore, plants were not inoculated with the leaf spotting fungi. The rust fungus was applied to plants 45 days after planting as described previously. The number of treatments included in this trial was reduced to six due to limitations in the amount of seed available. All fungicide treatments began 38 days after planting. The $1 \times$ rate of chlorothalonil was tested at five biweekly sprays (standard $1 \times$ treatment) on days $38,52,66,81$, and 95 , and at four triweekly applications ( $1 \times-21$ day treatment) on days $38,59,81$, and 98 . Also, five sprays of the $1 \times$ rate were applied on days $38,48,63,81$, and 95 according to an AU-Pnuts program adjusted to five rain events $\geq 2.5 \mathrm{~mm}$ (Jacobi and Backman, 1995). The $0.25 \times$ and $0.5 \times$ fungicidal rates were applied 10 (weekly) and seven times (10-day intervals) from day 38 .

\section{Management of epidemics with mixed fungicidal schedules}

Two experiments on peanuts were established on 2 June 1997 in areas planted to peanuts the previous year (Experiment A) or the previous 2 years (Experiment B) to subject peanut plants to different levels of inoculum of the leaf-spotting fungi. Plants were not inoculated with $P$. arachidis in the 1997 experiments. The following programs of fungicidal sprays were evaluated in 1997: $i$, the standard 1× rate of chlorothalonil, applied five times from days 35 to $88, i i$, the AU-Pnuts advisory system, which had six sprays from days 35 to 88 , iii, the systemic fungicide tebuconazole, applied four times between 35 and 77 days after planting, $i v$, the $0.25 \times$ rate of chlorothalonil, sprayed 15 times (twice-weekly) from day 35 to day $88, v$, the $0.5 \times$ rate of chlorothalonil, applied 
eight times (weekly) between days 35 and $88, v i$, the mixed-rate schedule I, which included six twice-weekly sprays of the $0.25 \times$ rate from day 35 to 53 plus three applications of the $1 \times$ rate on days 57,73 , and 84 , vii, the mixed schedule II, which comprised eight twice-weekly applications of the $0.25 \times$ rate between days 35 and 60 and three sprays of the $1 \times$ rate on days 63,77 , and 88 , viii, the mixed schedule III, which had three weekly sprays of the $0.5 \times$ rate from day 35 to 50 plus three applications of the $1 \times$ rate on days 57,73 , and 84 , and $i x$, the mixed spray program IV, with four weekly applications of the $0.5 \times$ rate between days 35 and 57 and three sprays of the $1 \times$ rate on days 63,77 , and 88 . The control of leaf-spot diseases and rust was evaluated two times a week from days 36 to 104 in the experiment A, and from days 36 to 87 in the experiment B. Plants were harvested on 3 October 1997 to determine pod yield.

\section{$\underline{\text { Statistical Analyses }}$}

The statistical analysis of the epidemic parameters $t_{0}, y_{0}, y_{\max }, r_{G N L}$, and AUDPC for the experiments on peanuts followed the same steps previously described for oats. The relationship of AUDPC to peanut yield was analyzed by linear or nonlinear regression. The effect of fungicidal spray programs on peanut yield was subjected to analysis of variance as implemented in SAS-PC. Upon significance, the treatment means were compared by the Duncan's Multiple Range Test at the $P=0.05$ type I error rate.

$\underline{\text { Results }}$

\section{Disease Efficiency of $P$. arachidis and $C$. arachidicola}

The disease efficiency (e) of $P$. arachidis (Fig. 5.1) on Florunner peanuts ranged from 2.9 to $4.82 \%$ on nonsprayed plants. Applications of the standard $1 \times$ rate of 


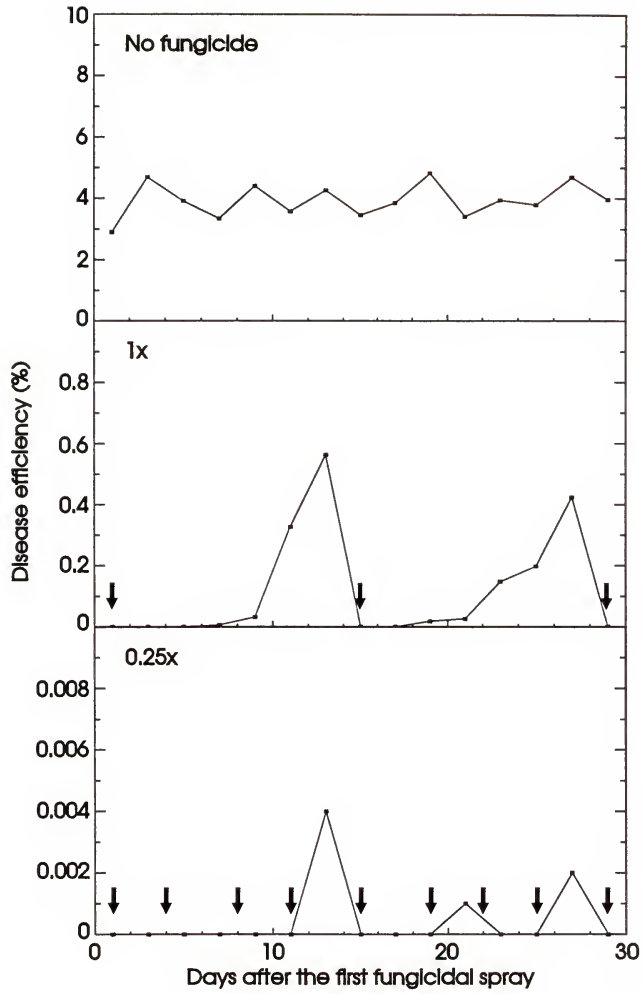

Fig. 5.1. Disease efficiency of Puccinia arachidis on peanuts, cultivar Florunner, subjected to $0 \times$ (no fungicide), $0.25 \times$, or $1 \times$ the label rate of chlorothalonil. Arrows indicate days on which fungicidal treatments were applied. Four plants in each treatment were inoculated daily with $4 \times 10^{4}$ uredospores $\mathrm{ml}^{-1}$. Experiment conducted in outdoor ambient conditions in 1995. 
chlorothalonil protected plants from infection for 6 days after the first fungicidal spray. From day 6 to 13 , however, the disease efficiency increased from 0.006 to $0.563 \%$ as the fungicidal deposit on the leaf surface decayed. Reapplication of the $1 \times$ rate on day 14 reduced $e$ to zero and resulted in a subsequent slower increase in disease efficiency (from 0.018 to $0.424 \%$ ) than that observed after the first fungicidal application. The lower variation in $e$ after the second spray might be associated with accumulation of fungicide on the leaf surface. In the $0.25 x$-rate treatment, reapplication of fungicide at every 3-4 days prevented an increase in disease efficiency, and the maximum $e$ was only $0.004 \%$.

Cercospora arachidicola had a disease efficiency on nonsprayed peanuts ranging from 0.73 to $3.25 \%$ (Fig. 5.2). After day $19, e$ decreased gradually as the viability of conidia obtained from diseased leaves stored in refrigerator was reduced. The disease efficiency of C. arachidicola in the $1 \times$-spray program increased from 0.003 to $0.161 \%$ after the first spray and from 0.001 to $0.066 \%$ following the second spray of fungicide. In the $0.25 \times$-rate treatment, the leaf spot fungus had several, little peaks of infection over time, and the highest $e$ value was only $0.005 \%$.

\section{Effect of Low Rates of Fungicide on Control of Disease and Yield in 1995}

Epidemics of leaf spots on nonsprayed plants in 1995 were initiated 56 days after planting, that is, 14 days after plants were inoculated with leaf-spotting fungi. Although the spore suspension used for inoculation had some conidia of $C$. personatum, early leaf spot predominated over late leaf spot in 1995. Late leaf spot was observed only at the end of the growing season and at low disease levels $\left(y_{f}=0.07\right)$. Peanut plants were inoculated with $P$. arachidis 63 days after planting and rust pustules were visible 10 days later. Even though peanut plants were inoculated with the rust fungus 3 weeks later than 


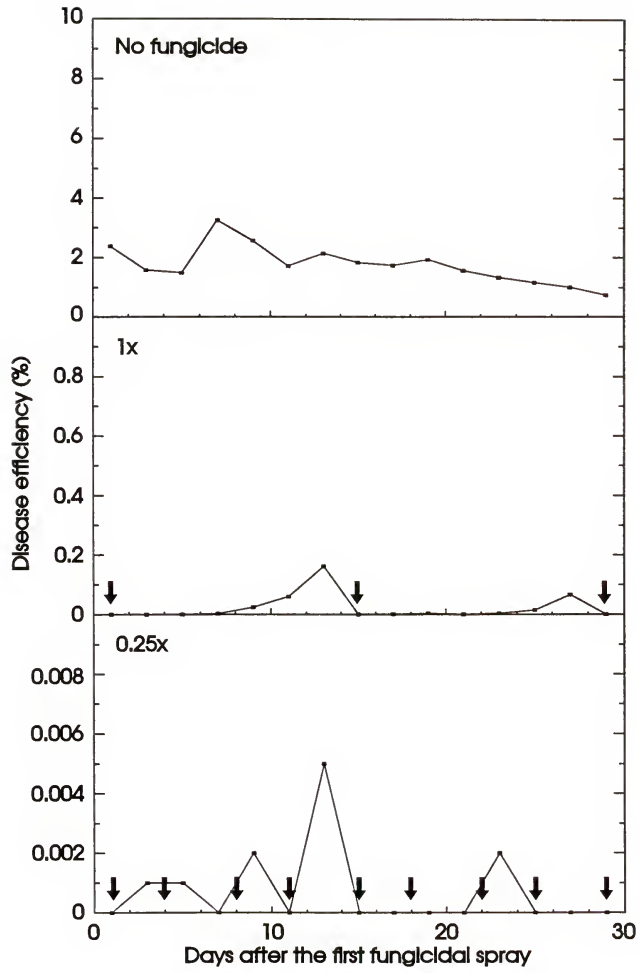

Fig. 5.2. Disease efficiency of Cercospora arachidicola on peanuts, cultivar Florunner, subjected to $0 \times$ (no fungicide), $0.25 \times$, or $1 \times$ the label rate of chlorothalonil. Arrows indicate days on which fungicidal treatments were applied. Four plants in each treatment were inoculated daily with $2 \times 10^{4}$ conidia $\mathrm{ml}^{-1}$. Experiment conducted in outdoor ambient conditions in 1995. 
inoculations with $C$. arachidicola and $C$. personatum, peanut rust progressed very fast and prevailed over leaf spot diseases. The final proportion of the disease complex $y_{f}=$ 0.7 ) was partitioned into 0.07 for late leaf spot, 0.17 for early leaf spot, and 0.46 for rust. The estimated epidemic rate $\left(r_{G N L}\right)$ of the disease complex on nonsprayed plants was 0.074 gompits day ${ }^{-1}$.

The estimated daily increment of disease proportion $\left(d_{y} / d_{t}\right)$ (Fig. 5.3-B) was very low (less than 0.001 ) from day 56 to 71 because of the slow development of early leaf spot. After day 73, however, the mixed epidemics of rust and leaf spots resulted in an increased increment of disease. The maximum $d_{y} / d_{t}(0.033)$ occurred around days 81 and 87.

The plot of disease proportions transformed by $-\ln [-\ln (y)]$ resulted in one epidemic wave (Fig. 5.3-C), which occurred from day 56 to day 71 and corresponded to the initial development of early leaf spot in the field. The progress of the mixed epidemics of rust and leaf spots that occurred after day 73 was nearly a straight line when transformed with gompits (Fig. 5.3-C).

Fungicidal treatments applied prior to the inoculation of peanut plants reduced the proportion of initial disease $\left(y_{0}\right)$ (Table 5.1 and Fig. 5.4-B). This reduction was significant $(P=0.05)$ even when only $10 \%(0.1 \times)$ of the label rate was used to control disease. Applications of $0.25 \times$ chlorothalonil twice a week delayed epidemic onset on additional 3 days. Initiation of fungicidal treatments 7 days after inoculation (1x-delay) had no effect on $y_{0}$. All spray programs, except $1 \times$-delay, also reduced the height of the epidemic waves, that is, the increase in disease between days 56 and 71 was lower on sprayed than on nonsprayed plants. 


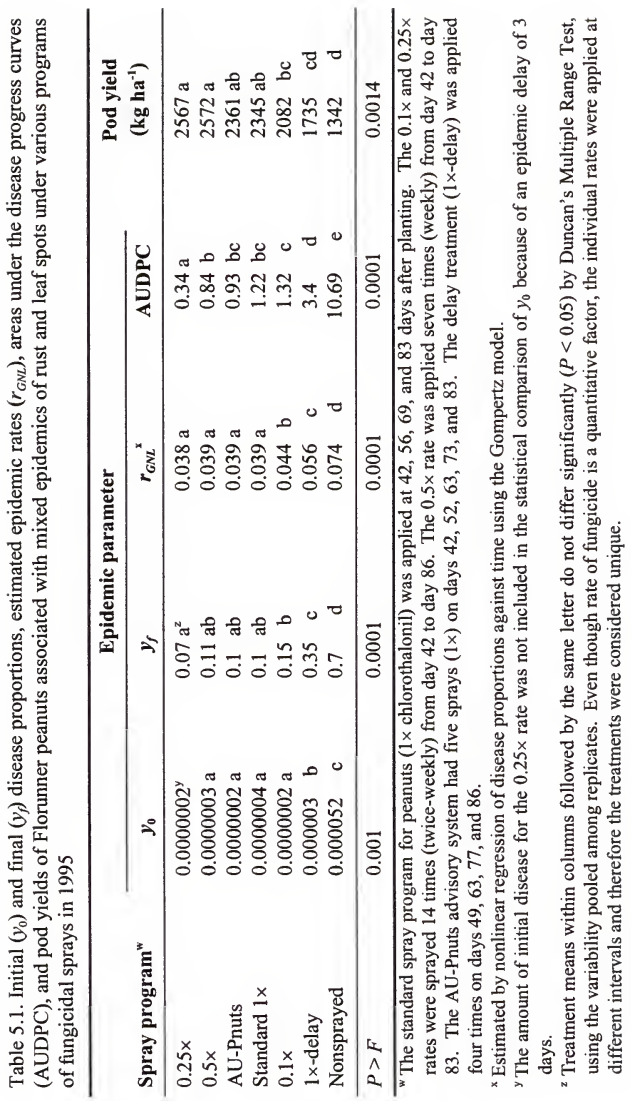




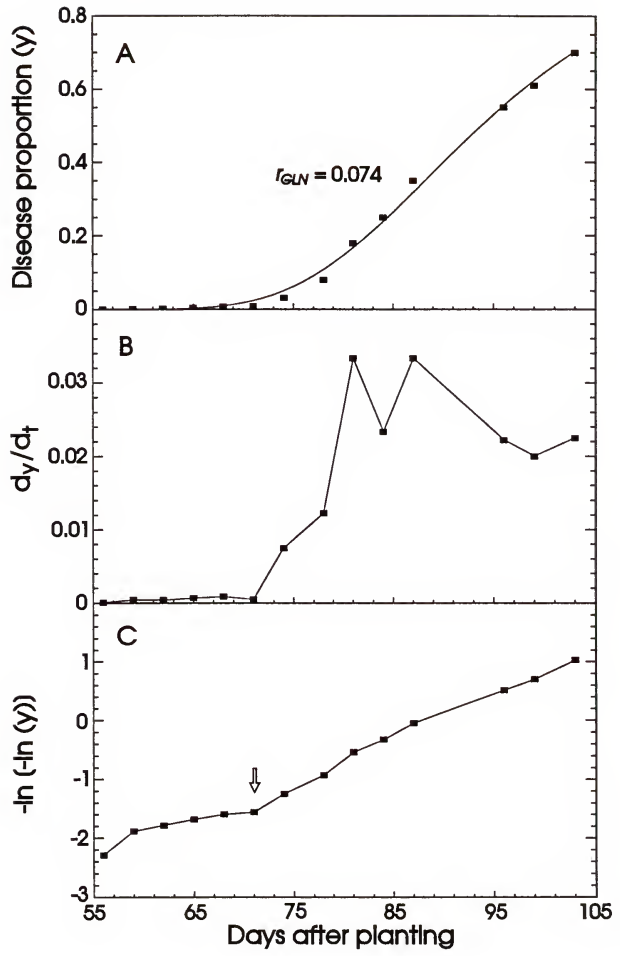

Fig. 5.3. Disease progress curve (A), estimated daily increment in disease severity $\left(d_{y} / d_{t}\right)$ (B), and disease wave (C) for a mixed epidemic of rust and leaf spots on nonsprayed Florunner peanuts in 1995 . The epidemic rate $\left(r_{G N L}\right)$ was estimated by nonlinear regression of disease proportions against time using the Gompertz model. The arrow indicates the end of the epidemic wave. 
Fig. 5.4. Disease progress curves (A) and disease waves (B) for mixed epidemics of rust and leaf spots on Florunner peanuts subjected to full- and reduced-rate programs of fungicidal sprays in 1995 . The standard spray program for peanuts $(1 \times$ chlorothalonil) was applied at $42,56,69$, and 83 days after planting. The delay treatment (1x-delay) was applied on days $49,63,77$, and 86 . The AU-Pnuts advisory system had five sprays (1×) on days $42,52,63,73$, and 83 . The $0.1 \times$ and $0.25 \times$ rates were sprayed 14 times (twiceweekly) from day 42 to day 86 . The $0.5 \times$ rate was applied seven times (weekly) from day 42 to day 83 . The epidemic rates $\left(r_{G N L}\right)$ were estimated by nonlinear regression of disease proportions against time using the Gompertz model. The arrow indicates the end of the epidemic wave. 


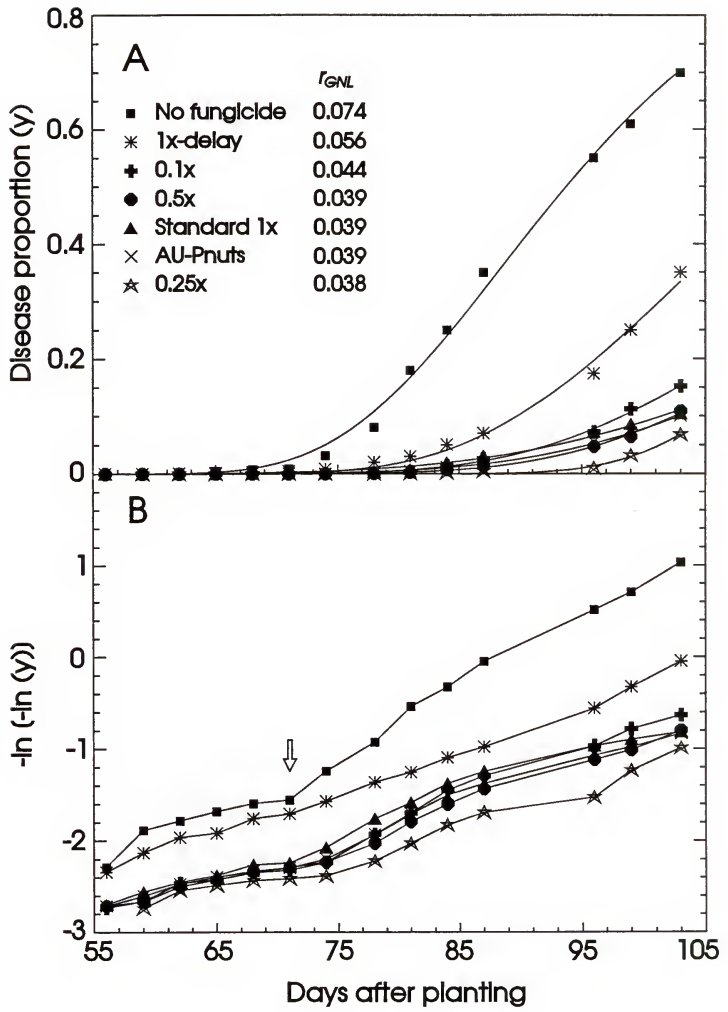


Plants sprayed with fungicide had epidemic rates $\left(r_{G N}\right)$ in the range of 0.038 to 0.056 gompits day ${ }^{-1}$, which were slower $(P=0.05)$ than that of the control treatment (0.074 gompits day $\left.{ }^{-1}\right)$ (Table 5.1 and Fig. 5.4-A). Although the epidemic rates and the final disease proportions $\left(y_{f}\right)$ were similar among the standard $1 \times$, AU-Pnuts, $0.5 \times$, and $0.25 \times$ treatments, the latter spray program had the lowest AUDPC (Table 5.1.). The overall best control of disease provided by twice-weekly sprays of $0.25 \times$ chlorothalonil was credited to the delay in epidemic onset mentioned previously. The $0.1 \times$ and $0.5 \times$ fungicidal rates provided control of rust and leaf spots comparable to the standard $1 \times$ treatment. Five applications of chlorothalonil according to the AU-Pnuts advisory system resulted in similar $y_{f}$ and AUDPC to those associated with four sprays of the same fungicide in the standard spray program. The 1x-delay treatment was the least efficacious of all spray programs. Compared to the standard $1 \times$ spray program initiated 42 days after planting, the $1 \times$-delay treatment resulted in significantly $(P=0.05)$ higher final disease (0.1 vs. 0.35 ), greater AUDPC (1.22 vs. 3.4 ), and a $26 \%$ loss in yield (2345 vs. $1735 \mathrm{~kg} \mathrm{ha}^{-1}$ ). Overall, pod yield was inversely related to AUDPC (Table 5.1). Peanut plants maintained with the $0.25 \times$-spray program $\left(2567 \mathrm{~kg} \mathrm{ha}^{-1}\right)$ yielded twice as much as the control plants (1342 $\left.\mathrm{kg} \mathrm{ha}^{-1}\right)$.

Management of Epidemics with Low Rates of Fungicide with Monoculture of Peanuts

When peanuts were planted in an area used for the same crop the previous year, an early and uniform occurrence of leaf spot diseases was observed. Although $C$. arachidicola had been the prevalent leaf-spotting fungus in the 1995 experiment, $C$. personatum caused more severe epidemics in 1996. The proportion of leaf area affected with the three diseases on nonsprayed plants increased from $y_{0}=0.00007$ to $y_{f}=0.92$ in 
64 days, which resulted in an estimated epidemic rate $\left(r_{G N L}\right)$ of 0.088 gompits day ${ }^{-1}$ (Fig. 5.5-A). Early leaf spot accounted for 0.08 , rust for 0.25 , and late leaf spot for 0.59 of the final disease proportion $\left(y_{f}=0.92\right)$ in 1996.

The predominance of leaf spots, especially late leaf spot, over peanut rust in 1996 was associated with high levels of primary inoculum by the leaf-spotting fungi and with the inoculation procedures used for the rust fungus. The inoculum of $P$. arachidis came from border plants inoculated on day 45 , and as such, peanut rust was not detected in the middle rows of each plot until 65-70 days after planting. At this point, the defoliation caused by leaf spots was already under way and less plant tissue remained available for the rust fungus to infect.

The estimated daily increments of disease severity $\left(d_{y} / d_{t}\right)$ (Fig. 5.5-B) increased fast in the early stages of the epidemic and reached a maximum proportion of 0.037 at 68 days after planting. Most of this disease increase was associated with the two leaf-spot diseases. A second, but lower peak in $d_{y} / d_{t}$ assessed on day 80 was related to the development of peanut rust. One epidemic wave resulted from plotting $-\ln [-\ln (y)]$ against time (Fig. 5.5-C). This wave lasted about 14 days and increased the disease proportion from 0.00007 to 0.058 . The second epidemic wave was not detected because of the long interval (from day 70 to 77 ) between disease assessments.

In this experiment, sprays of fungicide were started 38 days (AU-Pnuts, $0.25 \times$, $0.5 \times$, and $1 \times$ rates) or 45 days (systemic and delay treatments) after planting. Early and late leaf spots, however, were evenly distributed among peanut plants prior to the initiation of spray applications. Therefore, it was not possible to differentiate any effect of fungicides on epidemic onset or on the amount of initial disease. The statistical comparison of $y_{0}$, for example, was not significant among fungicidal spray programs 


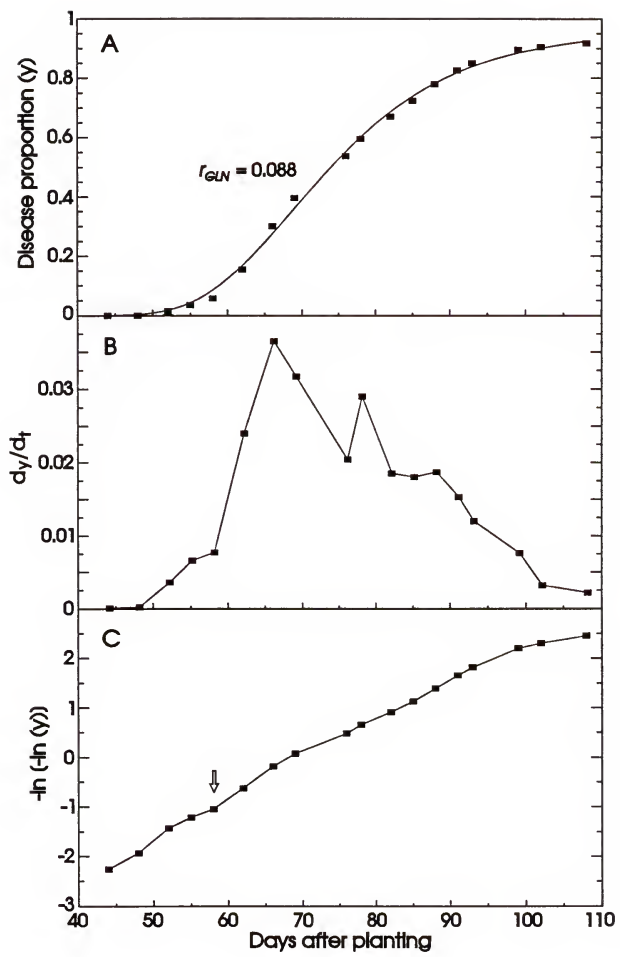

Fig. 5.5. Disease progress curve (A), estimated daily increments in disease severity $\left(d_{y} / d_{t}\right)$ (B), and disease wave (C) for a mixed epidemic of rust and leaf spots on nonsprayed Florunner peanuts in 1996. Experiment developed in field planted to peanuts the previous year. The epidemic rate $\left(r_{G N L}\right)$ was estimated by nonlinear regression of disease proportions against time using the Gompertz model. The arrow indicates the end of the epidemic wave. 
(Table 5.2). The major effect of fungicidal sprays on the epidemic waves was to reduce the height of the plotted epidemic waves. This effect was noticed from day 52 (Fig. 5.6$\mathrm{B}$ and 5.7-B) for treatments initiated 38 days after planting. The time interval between application of fungicide and the first evidence of disease control was about 14 days, which corresponded to the average latent period of peanut leaf spots in the field.

Although fungicides are usually applied on a calendar basis, weather-based schedules and new systemic fungicides are becoming more available. To compare the reduced-rate spray programs to fungicidal schedules used by peanut growers to time spray applications, a systemic (tebuconazole), a calendar- (standard $1 \times$ ), and a weatherbased treatment (AU-Pnuts) were included in this experiment. Tebuconazole, chlorothalonil (standard $1 \times$ rate), and AU-Pnuts were applied four, five, and six times at average intervals of 14,10 , and 14 days, respectively. Although sprays of tebuconazole were initiated 7 days later (day 45) than the standard 1x and AU-Pnuts spray programs (day 38), the effect of the spray applications on the epidemic wave appeared at the same time, about day 52 (Fig. 5.6-B). Therefore, while it took one latent period ( $p=14$ days) for the standard $1 \times$ and AU-Pnuts treatments to show some effect on the epidemic waves, the curative action of tebuconazole allowed this fungicide to reduce the height of the waves about $1 / 2 p$ after fungicidal application. Compared to the nonsprayed control, all three spray programs controlled the disease complex and reduced crop loss significantly $(P=0.05)$ (Table 5.2). Although the standard $1 \times$ treatment tended to be less efficacious than AU-Pnuts and the systemic fungicide, $y_{\rho}, r_{G N L}$, AUDPC, and pod yield did not differ statistically among these spray programs (Table 5.2 and Fig. 5.6-A).

Despite the similar effects of the $1 \times, 0.5 \times$, and $0.25 \times$ treatments on the epidemic waves (Fig. 5.7-B), the lower fungicidal rates provided better control of rust and leaf 


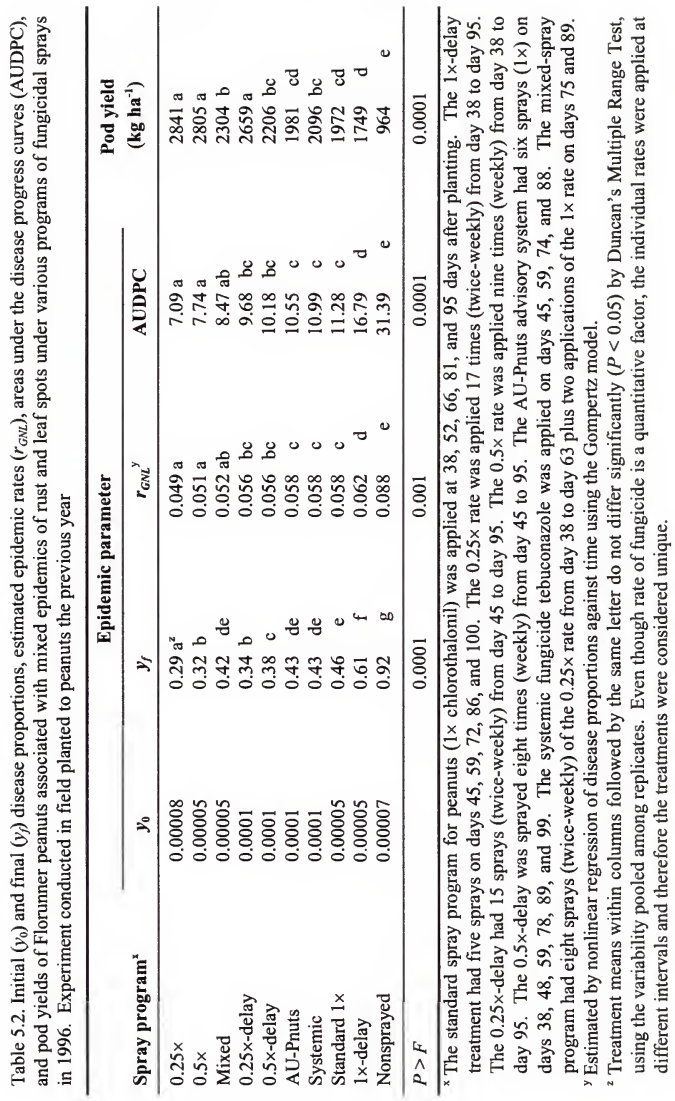


Fig. 5.6. Disease progress curves (A) and disease waves (B) for mixed epidemics of rust and leaf spots on Florunner peanuts subjected to various programs of fungicidal sprays in 1996. The standard spray program for peanuts $(1 \times$ chlorothalonil) was applied at 38,52 , 66,81 , and 95 days after planting. The AU-Pnuts advisory system had six sprays on days $38,48,59,78,89$, and 99 . The systemic fungicide tebuconazole was applied on days 45 , 59, 74, and 88. Experiment developed in field planted to peanuts the previous year. The epidemic rate $\left(r_{G N L}\right)$ was estimated by nonlinear regression of disease proportions against time using the Gompertz model. The arrow indicates the end of the epidemic wave. 


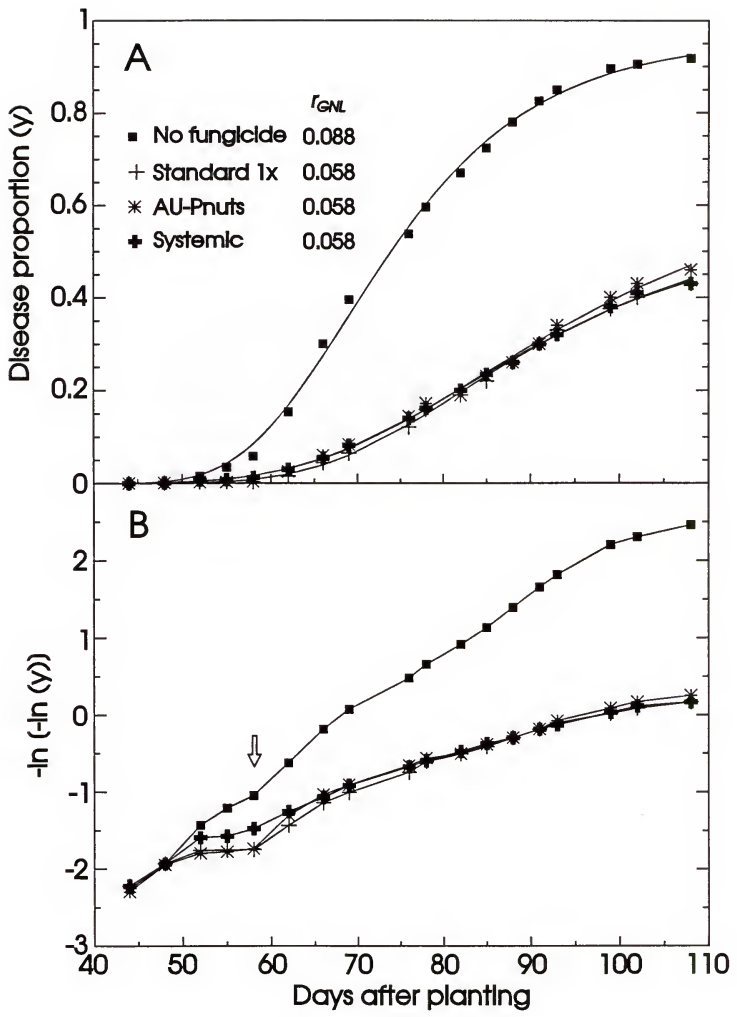


spots (Fig. 5.7-A). Sprays of $0.25 \times$ (twice-weekly) or $0.5 \times$ (weekly) slowed the epidemic rate significantly $(P=0.05)$, which resulted in lower final disease and lower AUDPC (Table 5.2). The pod yield of Florunner was $44 \%$ higher on plants sprayed with the reduced rates of fungicide. Overall, the $0.25 \times$ and $0.5 \times$ rates did not differ in the control of rust and leaf spots.

Initiation of spray applications at 45 instead of 38 days after planting affected the overall efficiency of fungicidal treatments. The delayed applications of fungicide had no effect (1×-delay, Fig. 5.8-B) or a low effect (0.5×, Fig. 5.9-B, and 0.25×, Fig. 5.10-B) on the epidemic waves. The final disease and the AUDPC were always higher $(P=0.05)$ in the delay treatments than in the spray programs initiated one week earlier (Table 5.2). The increase in AUDPC associated with delay of fungicidal sprays was markedly higher for the full rate than it was for the lower rates of fungicide.

The control of rust and leaf spots provided by the mixed-spray program, which included eight twice-weekly sprays of the $0.25 \times$ rate from days 38 to 63 , plus two applications of the $1 \times$ rate on days 75 and 89 , did not differ from that observed for the $0.25 \times$ treatment until 82 days after planting (Fig. 5.11-A). After day 82, the epidemic progressed faster in the mixed treatment as the interval between fungicidal sprays was lengthened. Although the $0.25 \times$ and the mixed treatment had similar AUDPCs, the pod yield of the latter spray program was significantly reduced $(P=0.05)$ (Table 5.2) by the increased disease severity in the late epidemic. Compared to the standard $1 \times$ spray schedule, however, the mixed spray program provided better control of disease and resulted in higher pod yield (Table 5.2). 
Fig. 5.7. Disease progress curves (A) and disease waves (B) for mixed epidemics of rust and leaf spots on Florunner peanuts subjected to a full- and reduced-rate programs of fungicidal sprays in 1996. The standard spray program for peanuts ( $1 \times$ chlorothalonil) was applied at $38,52,66,81$, and 95 days after planting. The $0.5 \times$ rate was sprayed nine times (weekly) from day 38 to day 95 . The $0.25 \times$ rate was applied 17 times (twiceweekly) from day 38 to day 95 . Experiment developed in field planted to peanuts the previous year. The epidemic rate $\left(r_{G N}\right)$ was estimated by nonlinear regression of disease proportions against time using the Gompertz model. The arrow indicates the end of the epidemic wave. 


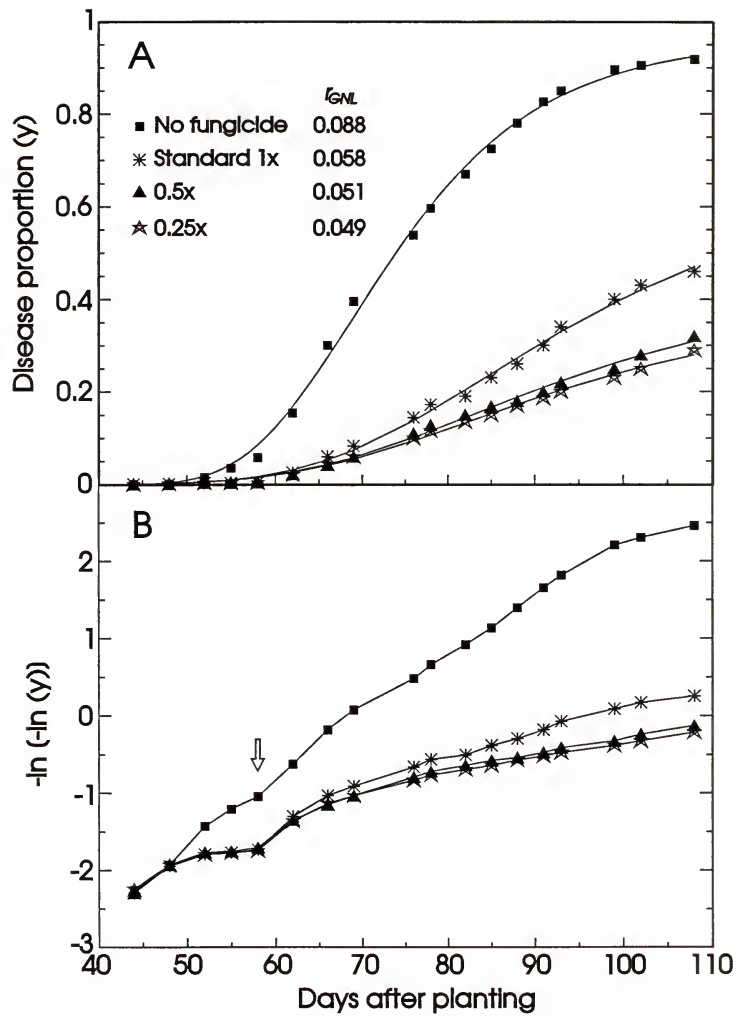


Fig. 5.8. Disease progress curves (A) and disease waves (B) for mixed epidemics of rust and leaf spots on Florunner peanuts subjected to sprays of $1 \times$ chlorothalonil initiated at two times after planting in 1996 . The standard $(1 \times)$ spray program for peanuts was applied at $38,52,66,81$, and 95 days after planting. The delay treatment (1×-delay) was sprayed on days $45,59,72,86$, and 100 . Experiment developed in field planted to peanuts the previous year. The epidemic rate $\left(r_{G N L}\right)$ was estimated by nonlinear regression od disease proportions against time using the Gompertz model. The arrow indicates the end of the epidemic wave. 


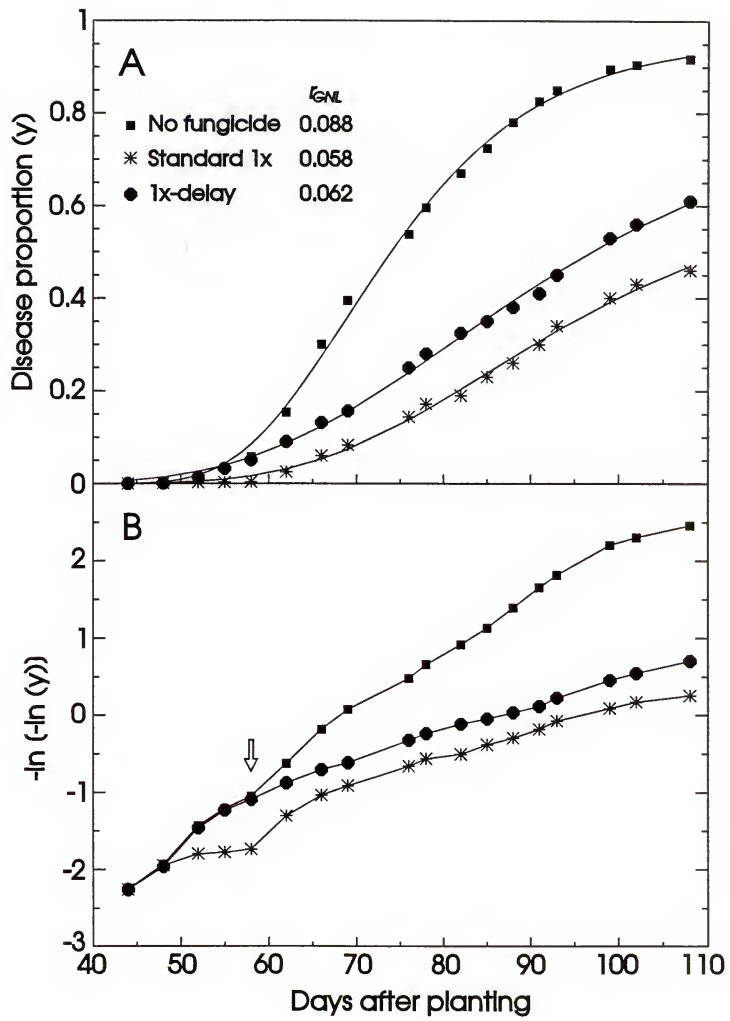


Fig. 5.9. Disease progress curves (A) and disease waves (B) for mixed epidemics of rust and leaf spots on Florunner peanuts subjected to sprays of $0.5 \times$ chlorothalonil initiated at two times after planting in 1996 . The $0.5 \times$ rate was applied nine times (weekly) from day 38 to day 95 . The $0.5 \times$-delay treatment had eight sprays (weekly) from day 45 to day 95 . Experiment developed in field planted to peanuts the previous year. The epidemic rate $\left(r_{G N L}\right)$ was estimated by nonlinear regression of disease proportions against time using the Gompertz model. The arrow indicates the end of the epidemic wave. 


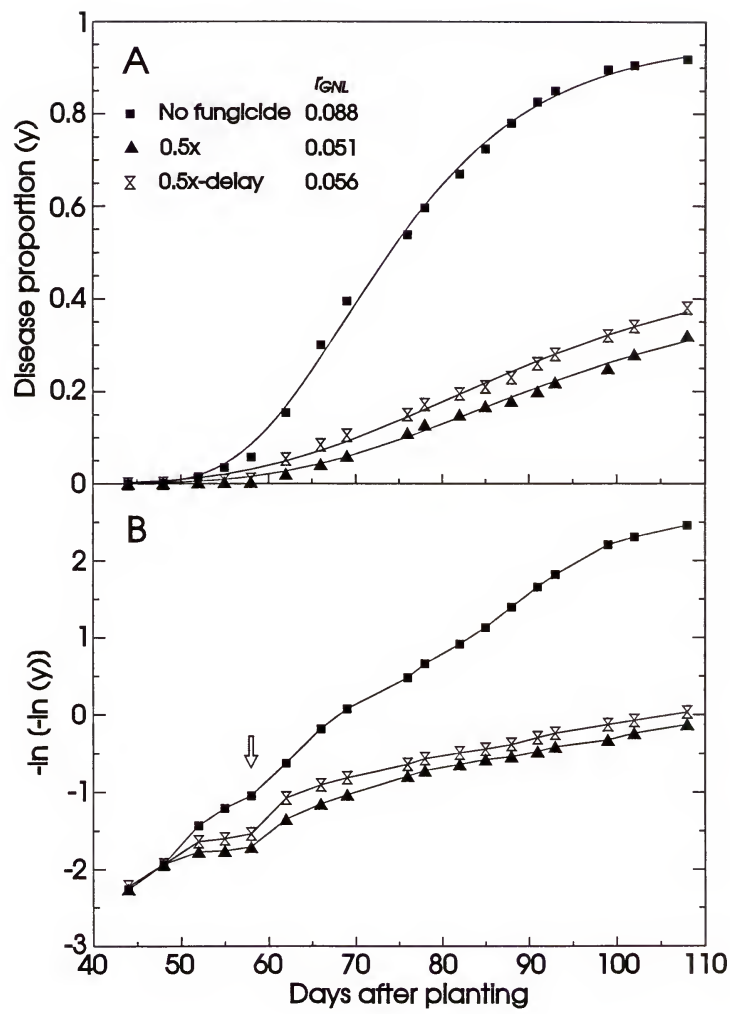


Fig. 5.10. Disease progress curves (A) and disease waves (B) for mixed epidemics of rust and leaf spots on Florunner peanuts subjected to sprays of $0.25 \times$ chlorothalonil initiated at two times after planting in 1996 . The $0.25 \times$ rate was applied 17 times (twice-weekly) from day 38 to day 95 . The $0.25 \times$-delay treatment had 15 sprays (twice-weekly) from day 45 to day 95 . Experiment developed in field planted to peanuts the previous year. The epidemic rate $\left(r_{G N L}\right)$ was estimated by nonlinear regression of disease proportions against time using the Gompertz model. The arrow indicates the end of the epidemic wave. 


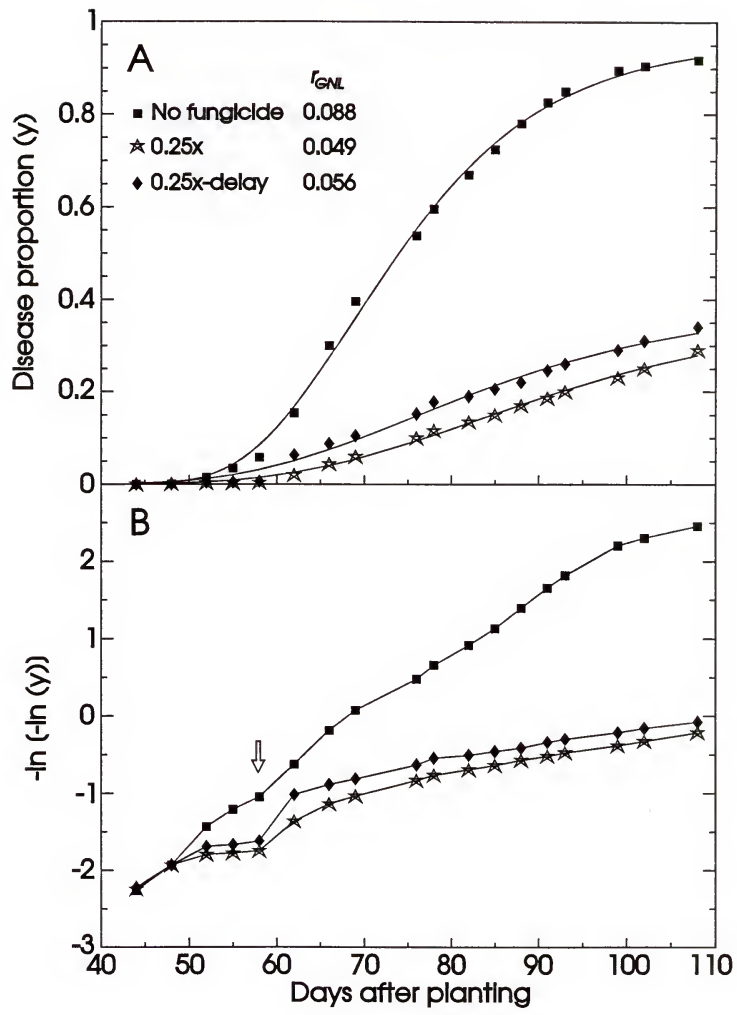


Fig. 5.11. Disease progress curves (A) and disease waves (B) for mixed epidemics of rust and leaf spots on Florunner peanuts subjected to single- or a multiple-rate spray program in 1996. The standard spray program for peanuts ( $1 \times$ chlorothalonil) was applied at 38 , $52,66,81$, and 95 days after planting. The $0.25 \times$ rate was applied 17 times (twiceweekly) from day 38 to day 95 . The mixed-rate program had eight sprays (twice-weekly) of the $0.25 \times$ rate from day 38 to day 63 plus two applications of the $1 \times$ rate on days 75 and 89. Experiment developed in field planted to peanuts the previous year. The epidemic rate $\left(r_{G N}\right)$ was estimated by nonlinear regression of disease proportions against time using the Gompertz model. The arrow indicates the end of the epidemic wave. 


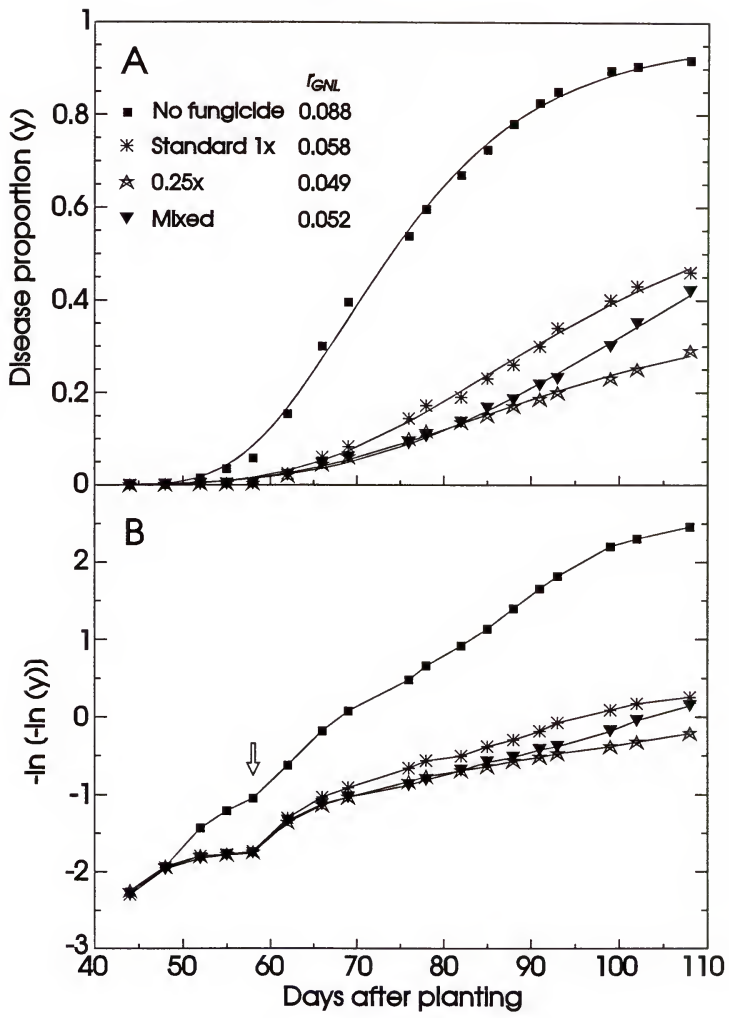


Management of Epidemics with Low Rates of Fungicides after Crop Rotation or Fallow

The low level of primary inoculum of $C$. arachidicola and C. personatum in this experiment resulted in a late and reduced occurrence of leaf spots. The first disease symptoms were observed 52 days after planting, that is, epidemic onset was at least 9 days later in this experiment than in that conducted under a monoculture of peanuts. On nonsprayed plants, the proportion of leaf spot diseases at 108 days after planting was less than 0.05 .

Peanut rust was the prevalent disease in this experiment. The first rust pustules were detected on nonsprayed plants on day 66,21 days after inoculation of plants in the border rows. Although rust appeared later than the leaf spots, the rust epidemic progressed faster and reached a higher proportion of final disease. For example, peanut rust accounted for 0.915 of the final disease proportion on nonsprayed plants $\left(y_{f}=0.96\right)$. The estimated daily increment of disease $\left(d_{y} / d_{t}\right)$ on nonsprayed plants was as high as 0.05 (Fig. 5.12-B) and resulted in an epidemic rate $\left(0.114\right.$ gompits day $\left.{ }^{-1}\right)$ that was faster in this experiment than in the previous trial $\left(0.088\right.$ gompits day $\left.{ }^{-1}\right)$. A flat disease wave (Fig. 5.12-C), which lasted 10 days, was observed for the epidemic on nonsprayed plants. Because of low levels of leaf spots in this experiment, the results presented below were assumed to represent the epidemic of peanut rust.

Among the most common fungicidal schedules used by peanut growers to time spray applications, the systemic fungicide tebuconazole provided better control of peanut rust than AU-Pnuts and the standard (1×) spray program (Fig. 5.13 and Table 5.3). Although these three treatments had similar amounts of initial disease, the systemic fungicide slowed the epidemic rate significantly $(P=0.05)$, which resulted in lower final disease and lower AUDPC. The peanut yield was increased significantly $(P=0.05)$ on 


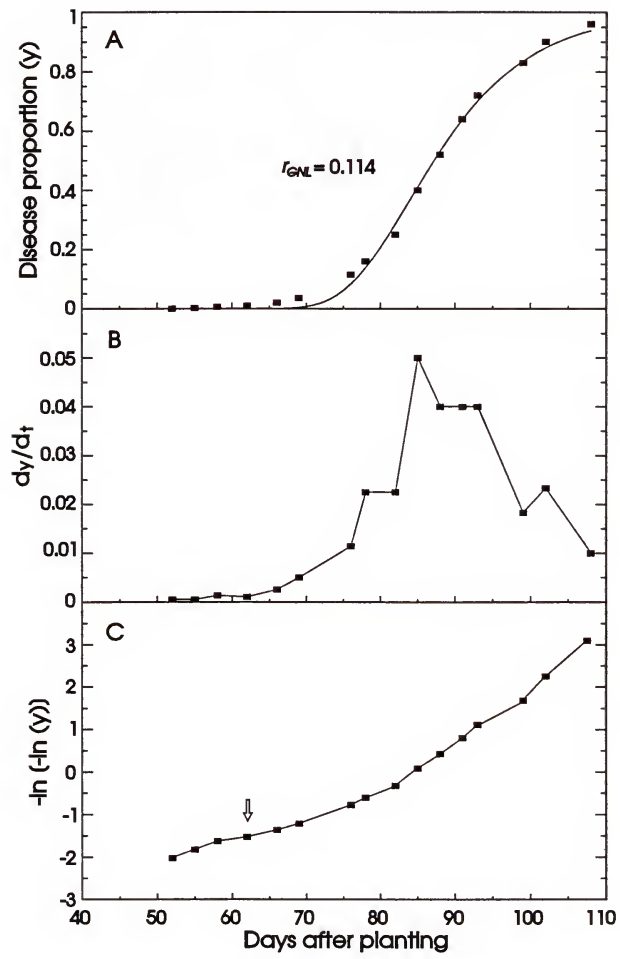

Fig. 5.12. Disease progress curve (A), estimated daily increment of disease severity $\left(d_{y} / d\right)$ (B), and disease wave (C) for a mixed epidemic of rust and leaf spots on nonsprayed Florunner peanuts in 1996. Experiment developed in field not previously planted to peanuts. The epidemic rate $\left(r_{G N L}\right)$ was estimated by nonlinear regression of disease proportions against time using the Gompertz model. The arrow indicates the end of the epidemic wave. 


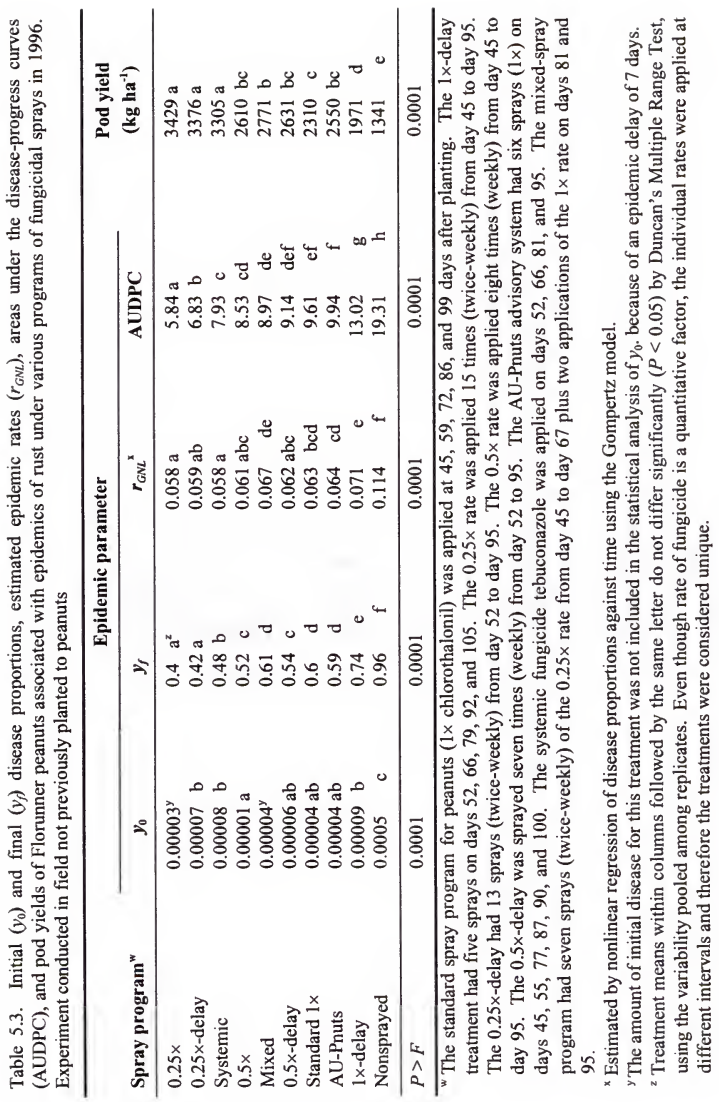


Fig. 5.13. Disease progress curves (A) and disease waves (B) for mixed epidemics of rust and leaf spots on Florunner peanuts subjected to various programs of fungicidal sprays in 1996. The standard spray program for peanuts ( $1 \times$ chlorothalonil) was applied at 45,59 , 72,86 , and 99 days after planting. The AU-Pnuts advisory system had six sprays of $1 \times$ chlorothalonil on days $45,55,77,87,90$, and 100 . The systemic fungicide tebuconazole was applied on days 52,66,81, and 95 . Experiment developed in field not previously planted to peanuts. The epidemic rate $\left(r_{G N L}\right)$ was estimated by nonlinear regression of disease proportions against time using the Gompertz model. The arrow indicates the end of the epidemic wave. 


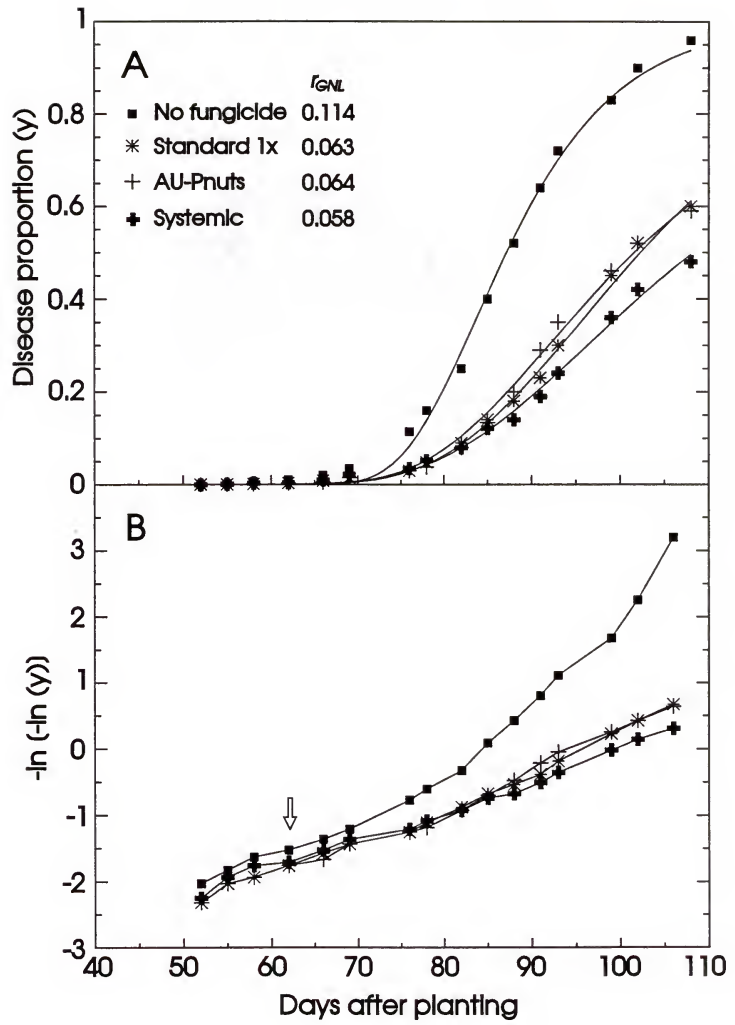


plants sprayed with tebuconazole compared to the AU-Pnuts and the standard $1 \times$ treatments. Six sprays of chlorothalonil according to the AU-Pnuts system did not reduce disease or increase yield significantly when compared to five sprays of the same fungicide at fixed intervals (standard $1 \times$ ).

Significant $(P=0.05)$ differences in disease control and pod yield were associated with applications of fungicide according to the $0.25 x$-spray program (Fig. 5.14 and Table 5.3). Compared to the standard $1 \times$ schedule, the lower-rate treatment delayed epidemic onset 6 days, reduced final disease $33 \%$ and AUDPC $39 \%$, and increased yield $48 \%$. The $0.5 \times$ rate also reduced $y_{f}$ and AUDPC significantly $(P=0.05)$ but pod yield did not differ from that of the standard treatment. Overall, twice-weekly sprays of the $0.25 \times$ provided better control of disease and higher pod yield than weekly sprays of the $0.5 \times$ rate.

Late initiation of fungicidal treatments had less impact on disease and yield in this experiment than it had in the trial under monoculture of peanuts. For example, no difference in $y_{0}$ was observed between the $0.5 \times$ and the $0.5 \times$-delay treatments (Fig. 5.16B and Table 5.3). Puccinia arachidis was inoculated in the border rows 45 days after planting. If an average latent period of 10 days is taken into account, infection of the test plants would not occur before day 55. Although the delay treatments were initiated 7 days after the regular spray schedules (day 45), all spray applications were carried out before plants were infected by rust. Although the $1 \times$ and $1 x$-delay treatments did not differ on $y_{0}$ (Table 5.3), plants in the delay treatment had significantly $(P=0.05)$ faster epidemic rate, higher final and total disease, and lower yield than those in the standard spray schedule. Such differences in disease and yield might be due to latent infections of leaf spots, which became symptomatic after the assessment of $y_{0}$ on the delay treatment. 
Fig. 5.14. Disease progress curves (A) and disease waves (B) for mixed epidemics of rust and leaf spots on Florunner peanuts subjected to full- and reduced-rate programs of fungicidal sprays in 1996. The standard spray program for peanuts (1× chlorothalonil) was applied at $45,59,72,86$, and 99 days after planting. The $0.5 \times$ rate was sprayed eight times (weekly) from day 45 to day 95 . The $0.25 \times$ rate was applied 15 times (twiceweekly) from day 45 to day 95 . Experiment developed in field not previously planted to peanuts. The epidemic rate $\left(r_{G N L}\right)$ was estimated by nonlinear regression of disease proportions against time using the Gompertz model. The arrow indicates the end of the epidemic wave. 


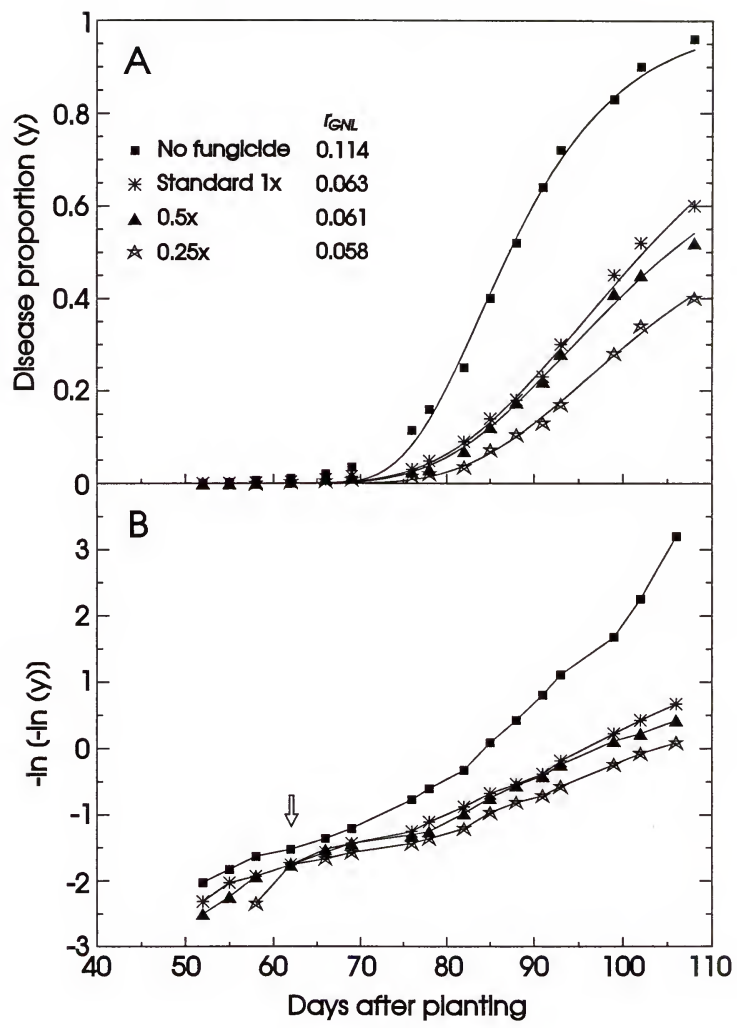


Fig. 5.15. Disease progress curves (A) and disease waves (B) for mixed epidemics of rust and leaf spots on Florunner peanuts subjected to sprays of $1 \times$ chlorothalonil initiated at two times after planting in 1996. The standard (1x) spray program for peanuts was applied at $45,59,72,86$, and 99 days after planting. The delay treatment (1x-delay) was sprayed on days $52,66,79,92$, and 105 . Experiment developed in field not previously planted to peanuts. The epidemic rate $\left(r_{G N L}\right)$ was estimated by nonlinear regression of disease proportions against time using the Gompertz model. The arrow indicates the end of the epidemic wave. 


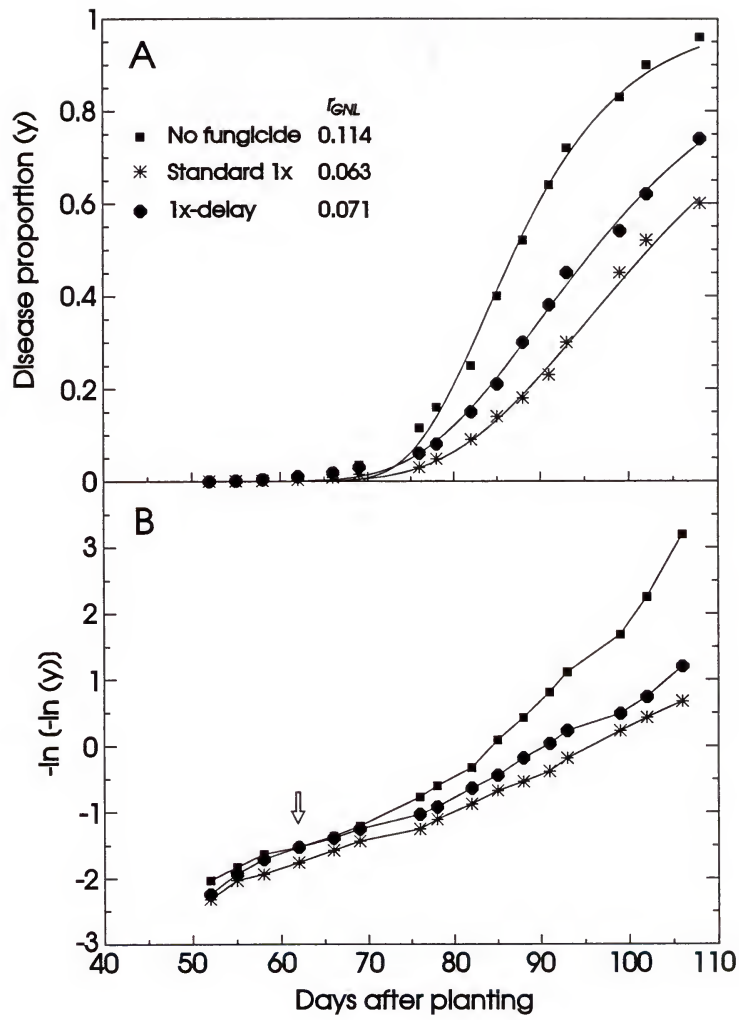


Fig. 5.16. Disease progress curves (A) and disease waves (B) for mixed epidemics of rust and leaf spots on Florunner peanuts subjected to sprays of $0.5 \times$ chlorothalonil initiated at two times after planting in 1996 . The $0.5 \times$ rate was applied eight times (weekly) from day 45 to day 95 . The $0.5 \times$-delay treatment had seven sprays (weekly) from day 52 to day 95. Experiment developed in field not previously planted to peanuts. The epidemic rate $\left(r_{G N L}\right)$ was estimated by nonlinear regression of disease proportions against time using the Gompertz model. The arrow indicates the end of the epidemic wave. 


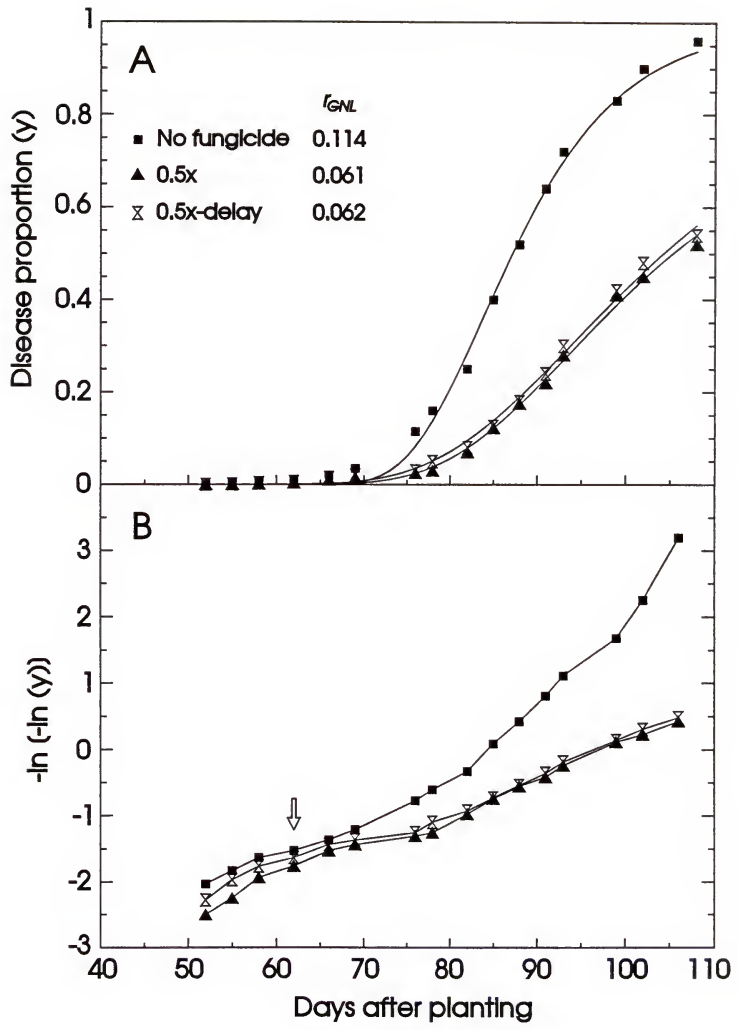


A major difference in the amount of initial disease existed only between the $0.25 \times$ and $0.25 \times$-delay treatments. The delay of epidemic onset provided by early sprays $(0.25 \times$ rate) did not occur when fungicidal applications were initiated 7 days later $(0.25 \times$-delay) (Fig. 5.17-B). The epidemic delay associated with early sprays of the $0.25 \times$ rate referred to the initial stages of leaf spots and not to rust.

Sprays of fungicide according to a mixed schedule (Fig. 5.18) resulted in poor control of disease. The epidemic rate, final disease proportion, and AUDPC of the mixed-spray treatment were significantly $(P=0.05)$ higher than those of the $0.25 \times$ rate (Table 5.3). In error, sprays of the full rate of fungicide were initiated late (day 81) after the last spray of the reduced rate (day 67). This gap in spray application coincided with the initial stages of the rust epidemic, which resulted in severe infection of plants in the mixed-spray treatment.

\section{Management of Epidemics with Low Rates of Fungicide on a Peanut Variety with Partial} Resistance to Disease

Because this experiment was established in a field planted to peanuts the previous year, the leaf-spotting fungi infected plants early in the season. Isolated leaf spots were detected as early as 30 days after planting. The early development of leaf spot diseases on nonsprayed plants was characterized by a well defined epidemic wave (Fig. 5.19-C), which lasted 14 days and increased disease 300-fold (from 0.00005 to 0.0166). Peanut rust was detected at day 69 , that is, there was a 24 -day interval between inoculation of plants in the border rows and the appearance of the first rust pustules in the plants of each plot. The estimated daily increment of disease $\left(d_{y} / d_{t}\right)$ for the mixed epidemic reached a maximum of 0.0275 on day 79 (Fig. 5.19-B) and then decreased gradually as the 
Fig. 5.17. Disease progress curves (A) and disease waves (B) for mixed epidemics of rust and leaf spots on Florunner peanuts subjected to sprays of $0.25 \times$ chlorothalonil initiated at two times after planting in 1996 . The $0.25 \times$ rate was applied 15 times (twice-weekly) from day 45 to day 95 . The $0.25 \times$-delay treatment had 13 sprays (twice-weekly) from day 52 to day 95 . Experiment developed in field not previously planted to peanuts. The epidemic rate $\left(r_{G N L}\right)$ was estimated by nonlinear regression of disease proportions against time using the Gompertz model. The arrow indicates the end of the epidemic wave. 


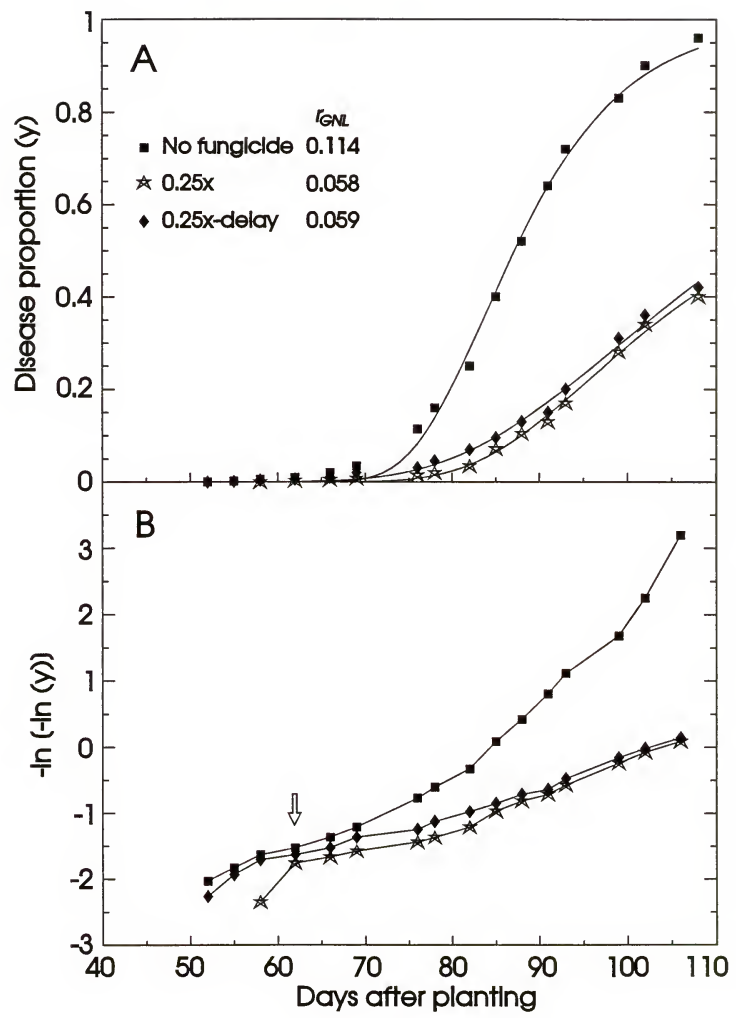


Fig. 5.18. Disease progress curves (A) and disease waves (B) for mixed epidemics of rust and leaf spots on Florunner peanuts subjected to single- or a multiple-rate spray program in 1996. The standard spray program for peanuts ( $1 \times$ chlorothalonil) was applied at 45 , $59,72,86$, and 99 days after planting. The $0.25 \times$ rate was applied 15 times (twiceweekly) from day 45 to day 95 . The mixed-rate program had seven sprays (twiceweekly) of the $0.25 \times$ rate from day 45 to day 67 plus two applications of the $1 \times$ rate on days 81 and 95 . Experiment developed in field not previously planted to peanuts. The epidemic rate $\left(r_{G N}\right)$ was estimated by nonlinear regression of disease proportions against time using the Gompertz model. The arrow indicates the end of the epidemic wave. 


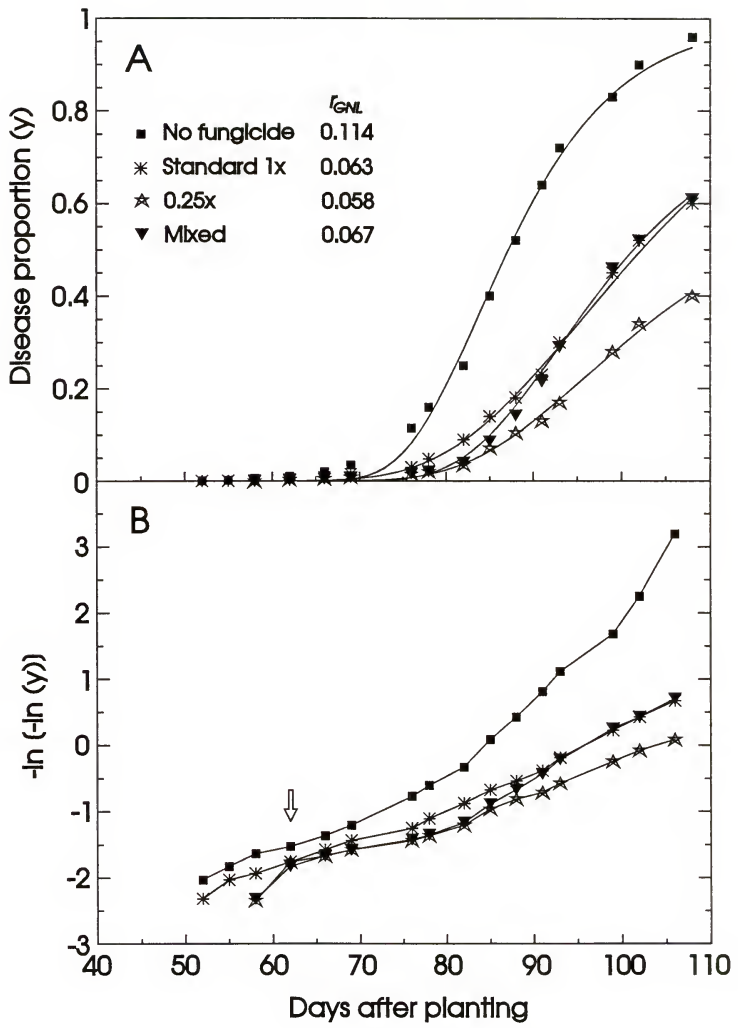




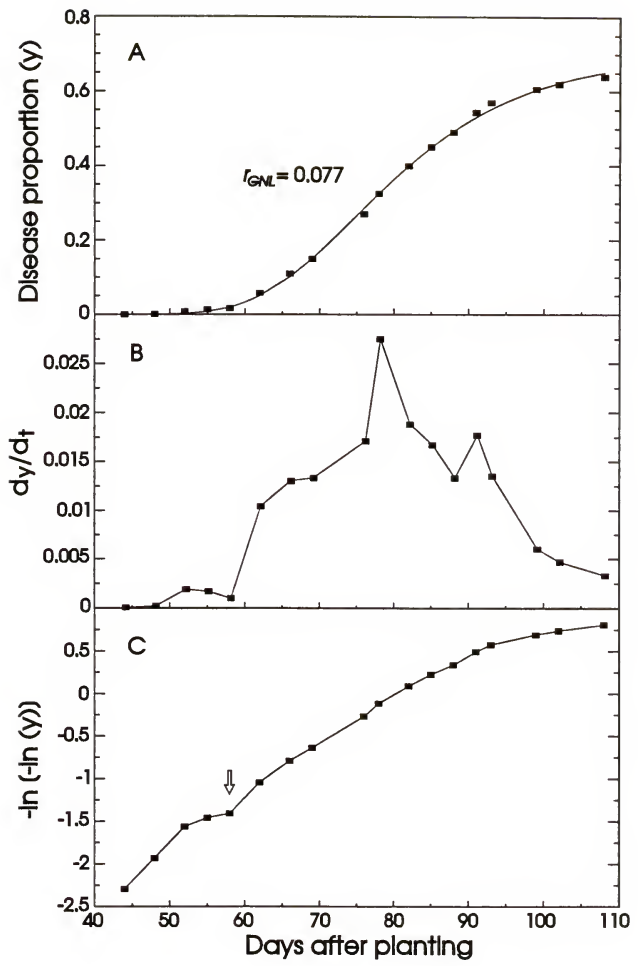

Fig. 5.19. Disease progress curve (A), estimated daily increments in disease severity $\left(d_{y} / d_{t}\right)(\mathrm{B})$, and disease wave (C) for a mixed epidemic of rust and leaf spots on nonsprayed Southern Runner peanuts in 1996. Experiment developed in field planted to peanuts the previous year. The epidemic rate $\left(r_{G N L}\right)$ was estimated by nonlinear regression of disease proportions against time using the Gompertz model. The arrow indicates the end of the epidemic wave. 
availability of healthy tissue diminished. The epidemic rate, including both rust and leaf spot diseases, was 0.077 gompits day ${ }^{-1}$ (Fig. 5.19-A). The final disease severity of the mixed epidemic (0.64) was 0.32 for rust, 0.3 for late leaf spot, and only 0.02 for early leaf spot disease.

Although Southern Runner has partial resistance to leaf-spot diseases, this cultivar did not differ from Florunner on the amount of initial disease when both cultivars were planted in the same field and exposed to similar amounts of inoculum. The $y_{0}$ on nonsprayed plants at 44 days after planting was 0.00005 on Southern Runner and 0.00007 on Florunner. However, the estimated daily increments of disease $\left(d_{y} / d_{t}\right)$ and the epidemic rate were slower in Southern Runner than in Florunner, which agreed with the rate-reducing characteristic of the first cultivar.

Since spray applications were initiated (day 38) after the leaf-spotting fungi infected plants, the various fungicidal treatments had no effect on epidemic onset or on the amount of initial disease (Fig. 5.20-B and Table 5.4). The major effect of fungicidal sprays was to reduce the basic infection rate $(R)$, which was demonstrated by the lower height of the plotted epidemic waves of sprayed plants. The reduction of the height of the epidemic wave was observed from day 52, that is, one latent period (about 14 days) after sprays of fungicide had been initiated.

Although various spray programs did not differ from each other in their epidemic rates, the final amount of disease and the AUDPC varied significantly $(P=0.05)$ among fungicidal treatments (Table 5.4). Applications of the $0.25 \times$ rate weekly resulted in lower AUDPC (7.79) than sprays of the standard $1 \times$ rate applied every 14 days (9.57). The lengthening of the spray interval from 14 (standard $1 \times)$ to 21 days $(1 \times-21$ days) reduced 


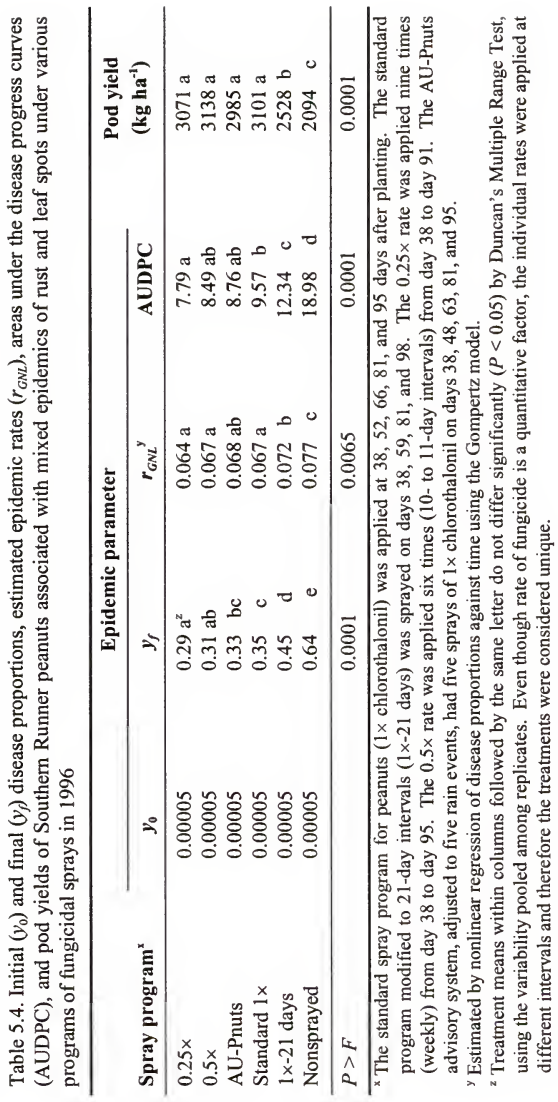


Fig. 5.20. Disease progress curves (A) and disease waves (B) for mixed epidemics of rust and leaf spots on Southern Runner peanuts subjected to various programs of fungicidal sprays in 1996. The standard spray program for peanuts $(1 \times$ chlorothalonil) was applied at $38,52,66,81$, and 95 days after planting. The standard program modified to 21-day intervals ( $1 \times-21$ days) was sprayed on days $38,59,81$, and 98 . The $0.25 \times$ rate was applied nine times (weekly) from day 38 to day 95 . The $0.5 \times$ rate program had six applications (10- to 11-day intervals) from day 38 to day 91 . The AU-Pnuts advisory system, adjusted to five rain events, had five sprays of $1 \times$ chlorothalonil on days 38,48 , 63,81 , and 95 . Experiment developed in field planted to peanuts the previous year. The epidemic rate $\left(r_{G N L}\right)$ was estimated by nonlinear regression of disease proportions against time using the Gompertz model. The arrow indicates the end of the epidemic wave. 


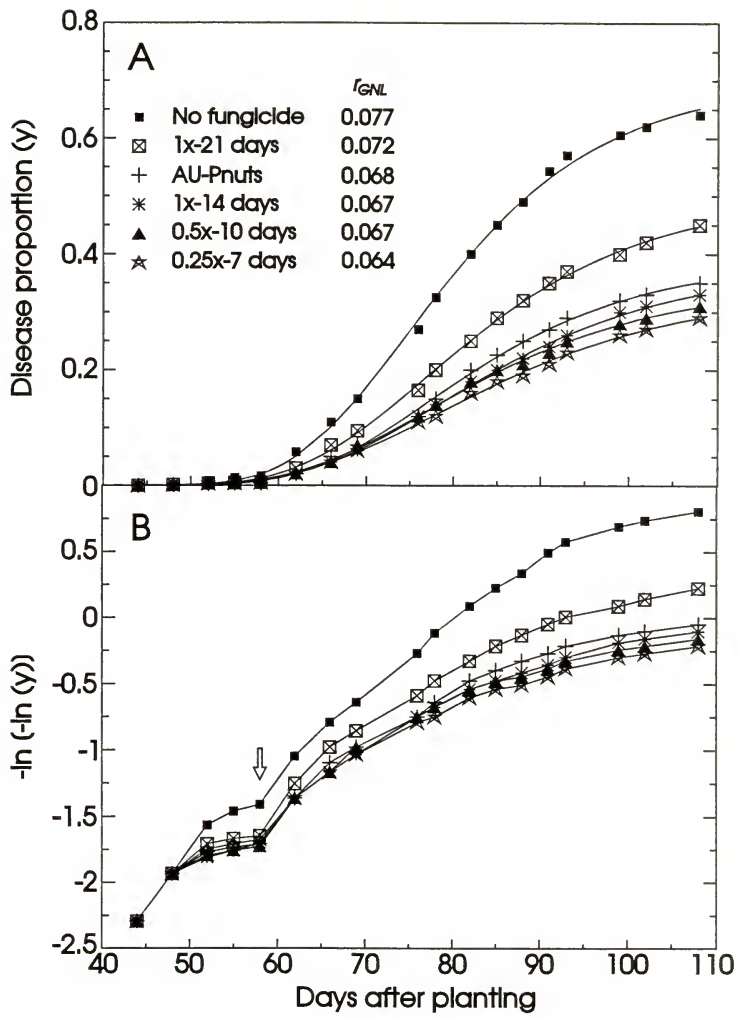


the control efficacy of the full rate of fungicide. The $0.5 \times$ rate, applied at 10 - to 11 -day intervals, did not differ in disease control and pod yield from the standard $1 \times$. However, the lower rate $(0.5 \times)$ reduced AUDPC significantly $(P=0.05)$ when compared to sprays of the full rate in the 21-day schedule. With the AU-Pnuts advisory system, modified to include five rain events, five sprays of fungicide were scheduled at an average interval of 14 days. This schedule reduced AUDPC significantly in relation to sprays of $1 \times$ every 21 days.

\section{Management of Epidemics with Mixed Spray Schedules}

Because the 1997 experiments on peanuts were conducted in areas planted to the same crop the previous year (1997-A) or the previous two years (1997-B), different amounts of initial disease $\left(y_{0}\right)$ were observed in these experiments. The $y_{0}$ averaged over all fungicidal spray programs was lower in 1997-A (0.0013) than in 1997-B (0.005). In both experiments, the late leaf spot prevailed and was equally distributed among the field plots. Early leaf spot was rarely seen in 1997.

The epidemic of peanut rust that developed from early August was not expected since the plants were not inoculated with $P$. arachidis in 1997. Pustules of peanut rust were initially detected in plots located on the west and southwest sides of the experiments, close to volunteer peanut plants. Although no pustules were observed in the plants sampled, it is possible that rust fungus overwintered in the volunteer peanuts grown in or near the experiment area. Peanut rust spread fast over all field plots, but the final proportion of this disease was low compared to late leaf spot. On nonsprayed plants, the $y_{f}$ for rust were less than 0.16 in 1997-A and less than 0.09 in 1997-B. Under

the same circumstances, the late leaf spot reached disease proportions equal to 0.78 in 
1997-A and 0.67 in 1997-B. In the 1997-B experiment, the disease assessments were discontinued at 87 days after planting because raccoons caused severe damage to the test plants.

Despite the lower $y_{0}$ observed in 1997-A, the epidemic progressed faster in this experiment $\left(r_{G N L}=0.074\right)$ than in 1997-B $\left(r_{G N L}=0.06\right)$. On day 87 , for example, the disease proportion on nonsprayed plants in 1997-A (0.75) was as high as that measured in 1997-B (0.76) (Figs. 5.21-A and 5.22-A). The daily increase of disease severity $\left(d_{y} / d_{t}\right)$ was higher in 1997-B between days 35 and 65 (Fig. 5.22-B). After day 65, however, a major increment of disease occurred in 1997-A (Fig. 5.21-B). In both experiments, the highest $d_{y} / d_{t}$ values were observed about 75 days after planting and corresponded to the simultaneous development of late leaf spot and rust. The plot of the disease proportions transformed with gompits resulted in two epidemic waves (Fig. 5.21-C and 5.22-C), which occurred between days 36 and 50, and between days 50 and 65 in both experiments. These epidemic waves corresponded to late leaf spot only, since peanut rust had not yet been detected in the field. In 1997-A, the epidemic increased 23-fold (from 0.001 to 0.023 ) during the first wave and 9-fold (from 0.023 to 0.206 ) during the second wave. In 1997-B, the first epidemic wave increased the disease proportion 16-fold (from 0.005 to 0.08 ) and the second wave only 4 -fold (from 0.08 to 0.335 ).

Significant differences $(P=0.05)$ in control of the disease complex were observed among the standard $1 \times$, the AU-Pnuts, and the systemic treatment (Tables 5.5 and 5.6; Figs. 5.23 and 5.24). Four sprays of the systemic fungicide tebuconazole resulted in the lowest $r_{G N L}, y_{f}$ and AUDPC among all spray schedules. The systemic activity of tebuconazole also allowed this fungicide to reduce the height of first epidemic wave in the 1997-B experiment (Fig. 5.23-B). Applications of chlorothalonil according to the 


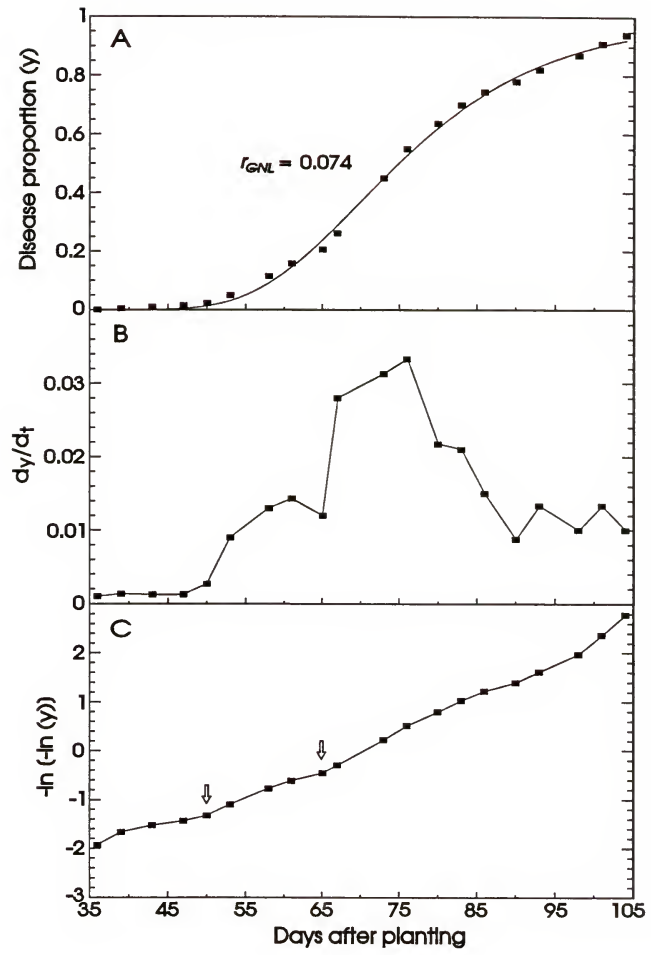

Fig. 5.21. Disease progress curve (A), estimated daily increments in disease severity $\left(d_{y} / d_{t}\right)(\mathrm{B})$, and disease wave (C) for a mixed epidemic of rust and leaf spots on nonsprayed Florunner peanuts in 1997. Experiment developed in field planted to peanuts the previous year. The epidemic rate $\left(r_{G N L}\right)$ was estimated by nonlinear regression of disease proportions against time using the Gompertz model. Arrows indicate the ends of epidemic waves. 


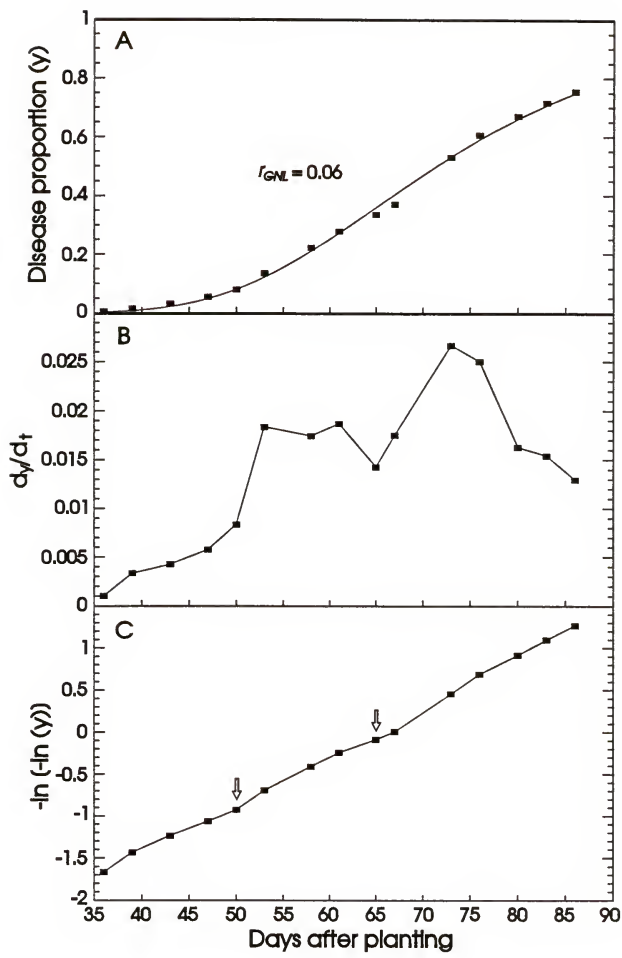

Fig. 5.22. Disease progress curve (A), estimated daily increments in disease severity $\left(d_{y} / d_{t}\right)(\mathrm{B})$, and disease wave (C) for a mixed epidemic of rust and leaf spots on nonsprayed Florunner peanuts in 1997. Experiment developed in field planted to peanuts the previous two years. The epidemic rate $\left(r_{G N L}\right)$ was estimated by nonlinear regression of disease proportions against time using the Gompertz model. Arrows indicate the ends of epidemic waves. 


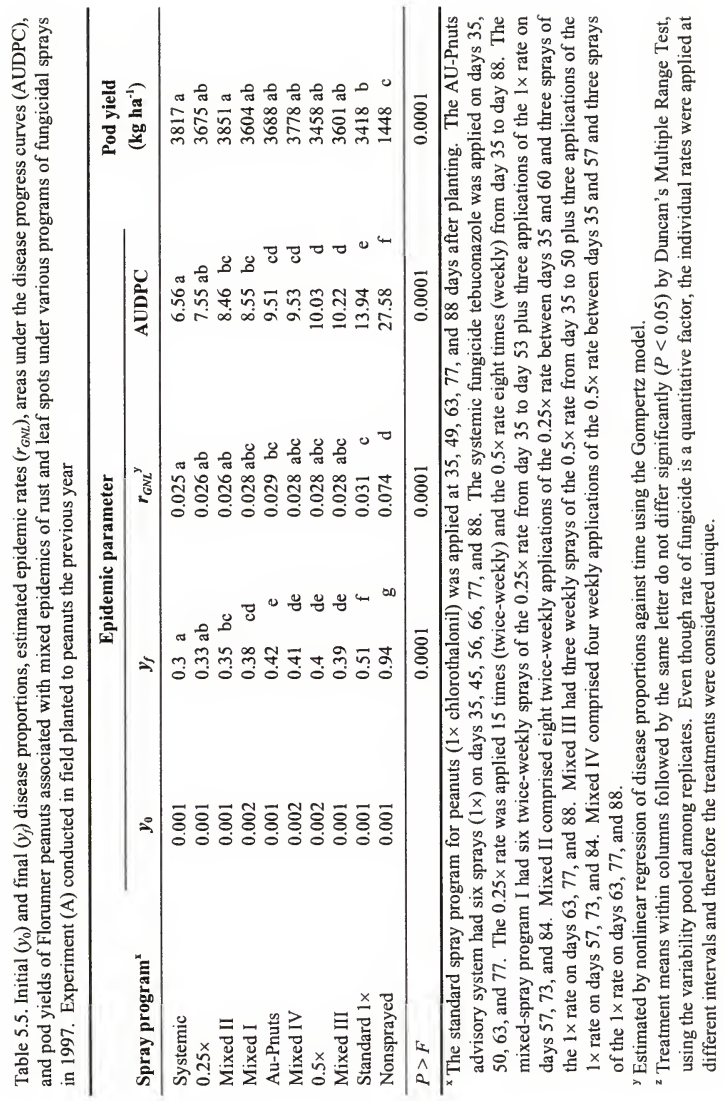




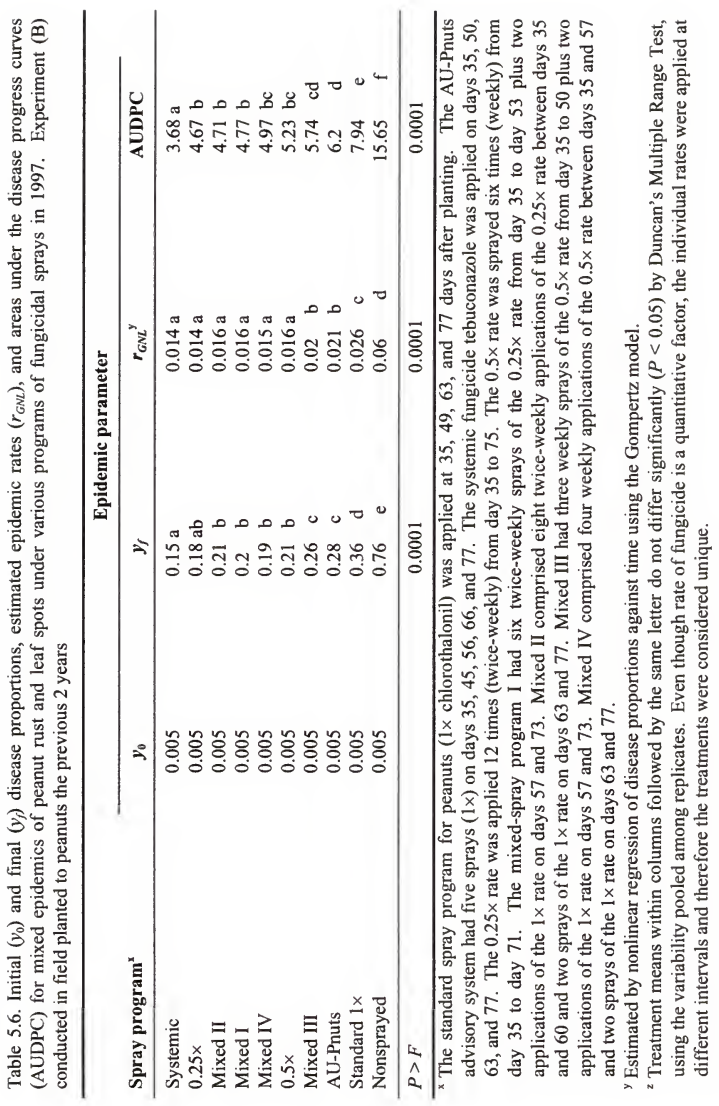


Fig. 5.23. Disease progress curves (A) and disease waves (B) for mixed epidemics of rust and leaf spots on Florunner peanuts subjected to various programs of fungicidal sprays in 1997. The standard spray program for peanuts ( $1 \times$ chlorothalonil) was applied at 35,49 , 63,77 , and 88 days after planting. The AU-Pnuts advisory system had six sprays of $1 \times$ chlorothalonil on days $35,45,56,66,77$, and 88 . The systemic fungicide tebuconazole was applied on days $35,50,63$, and 77 . Experiment developed in field planted to peanuts the previous year. The epidemic rates $\left(r_{G N L}\right)$ were estimated by nonlinear regression of disease proportions against time using the Gompertz model. Arrows indicate the ends of epidemic waves. 


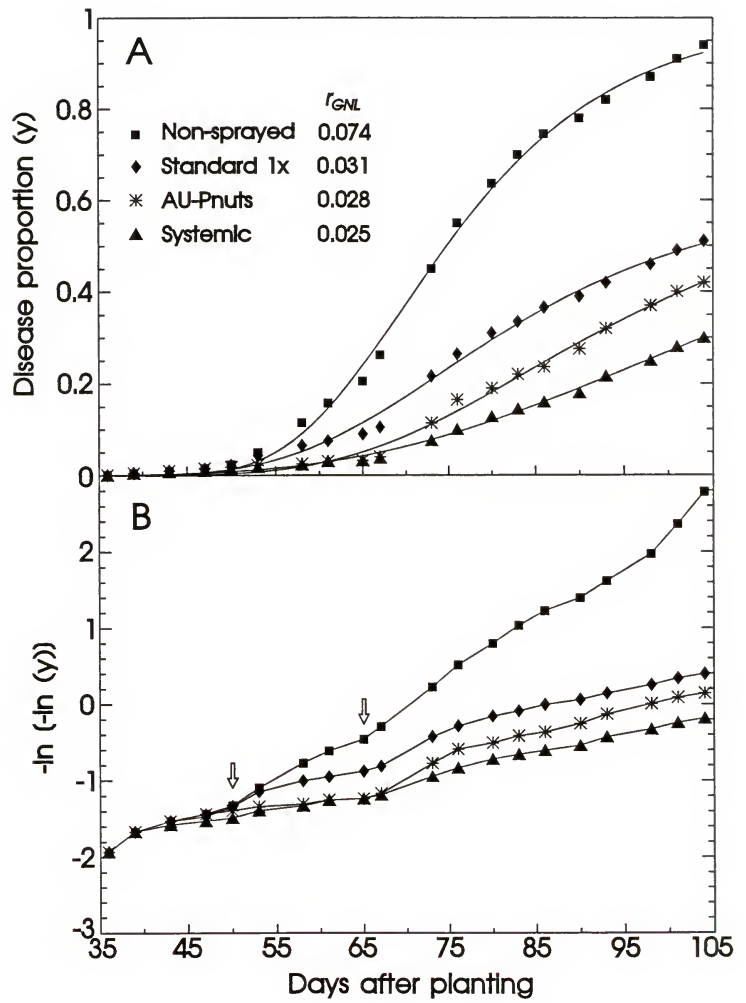


Fig. 5.24. Disease progress curves (A) and disease waves (B) for mixed epidemics of rust and leaf spots on Florunner peanuts subjected to various programs of fungicidal sprays in 1997. The standard spray program for peanuts ( $1 \times$ chlorothalonil) was applied at 35,49 , 63, and 77 days after planting. The AU-Pnuts advisory system had five sprays of $1 \times$ chlorothalonil on days $35,45,56,66$, and 77 . The systemic fungicide tebuconazole was applied on days $35,50,63$, and 77 . Experiment developed in field planted to peanuts the previous 2 years. The epidemic rates $\left(r_{G N L}\right)$ were estimated by nonlinear regression of disease proportions against time using the Gompertz model. Arrows indicate the ends of epidemic waves. 


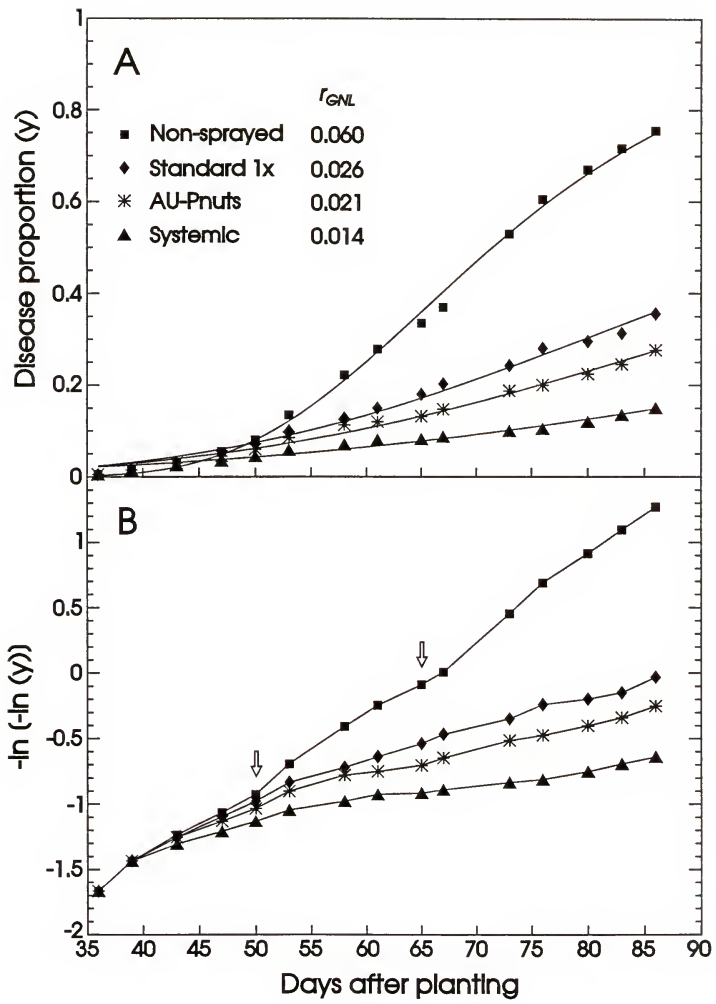


standard $1 \times$ and the AU-Pnuts spray schedules affected disease development after day 50, that is, one latent period ( $p=14$ days) after the first spray application. Therefore, the initial effect of the standard $1 \times$ and AU-Pnuts was to reduce the second epidemic wave, which occurred from day 50 to day 65 (Figs. 5.23-B and 5.24-B). The $y_{f}$ and AUDPC were lower on plants sprayed according to AU-Pnuts than on plants subjected to the standard spray schedule, especially in the 1997-A experiment. It should be noted that the full rate of chlorothalonil was sprayed six times in the AU-Pnuts treatment and five times in the standard spray schedule.

Modification of the standard biweekly sprays of $1 \times$ chlorothalonil to allow for weekly $(0.5 \times$ rate $)$ or twice-weekly $(0.25 \times$ rate $)$ applications of reduced fungicidal rates improved the control of the disease complex significantly $(P=0.05)$. Plants sprayed with $0.25 \times$ and $0.5 \times$ chlorothalonil had lower $y_{f}$ and AUDPC than plants subjected to the standard spray schedule (Table 5.5 and Figs. 5.25 and 5.26). The low rates of chlorothalonil also reduced the second epidemic wave over the standard treatment. Differences in disease control $\left(\gamma_{f}\right.$ and AUDPC) between the low rates $(0.25 \times$ and $0.5 \times)$ of fungicide occurred in 1997-A and were attributed to a higher effect of the $0.25 \times$ rate on the second epidemic wave (Fig. 5.25-B).

In the 1997 experiments on peanuts, the first and second applications of the standard $1 \times$ rate of chlorothalonil were replaced by weekly $(0.5 \times$ rate $)$ or twice-weekly ( $0.25 \times$ rate) sprays of lower rates of the same fungicide. The mixed schedules I and II included six and eight sprays of the $0.25 \times$ rate, respectively, while mixed III and IV had six and eight applications of the $0.5 \times$ rate, respectively. Overall, the mixed-rate schedules improved the control of disease significantly $(P=0.05)$ over the standard $1 \times$ 
Fig. 5.25. Disease progress curves (A) and disease waves (B) for mixed epidemics of rust and leaf spots on Florunner peanuts subjected to full- and reduced-rate programs of fungicidal sprays in 1997 . The standard spray program for peanuts (1× chlorothalonil) was applied at $35,49,63,77$, and 88 days after planting. The $0.25 \times$ and $0.5 \times$ rates were sprayed 15 times (twice-weekly) and eight times (weekly) from day 35 to day 88 . Experiment developed in field planted to peanuts the previous year. The epidemic rates $\left(r_{G N}\right)$ were estimated by nonlinear regression of disease proportions against time using the Gompertz model. Arrows indicate the ends of epidemic waves. 


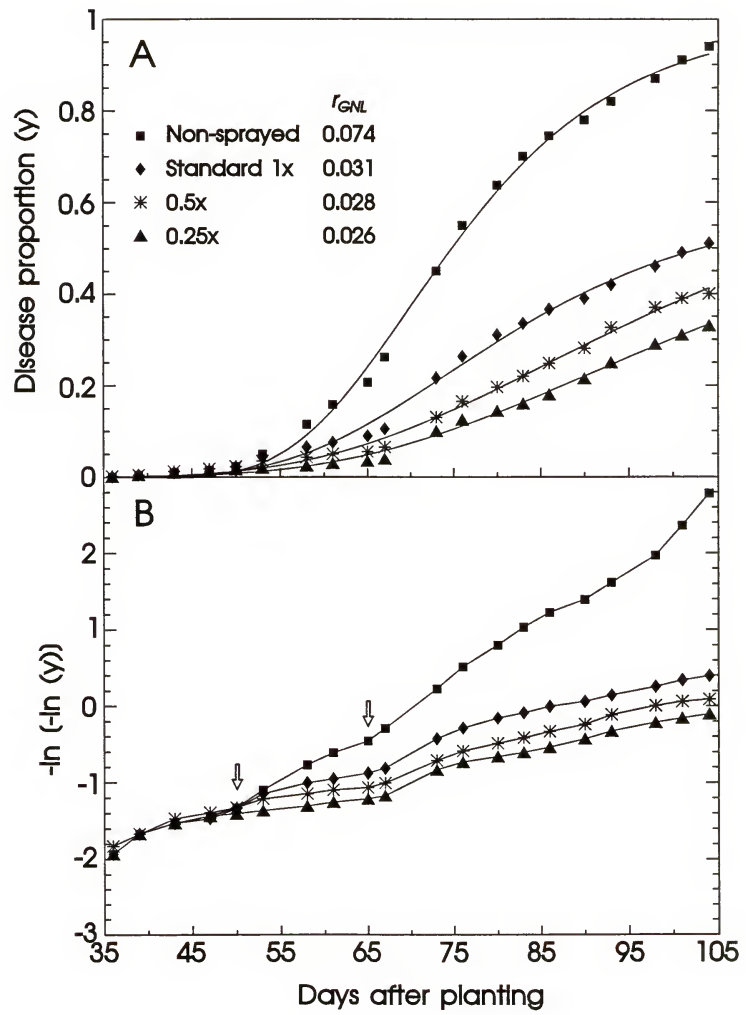


Fig. 5.26. Disease progress curves (A) and disease waves (B) for mixed epidemics of rust and leaf spots on Florunner peanuts subjected to full- and reduced-rate programs of fungicidal sprays in 1997. The standard spray program for peanuts ( $1 \times$ chlorothalonil) was applied at $35,49,63$, and 77 days after planting. The $0.25 \times$ rate was sprayed 12 times (twice-weekly) from day 35 to 75 . The $0.5 \times$ rate was applied six times (weekly) from day 35 to day 71 . Experiment developed in field planted to peanuts the previous 2 years. The epidemic rates $\left(r_{G N L}\right)$ were estimated by nonlinear regression of disease proportions against time using the Gompertz model. Arrows indicate the ends of epidemic waves. 


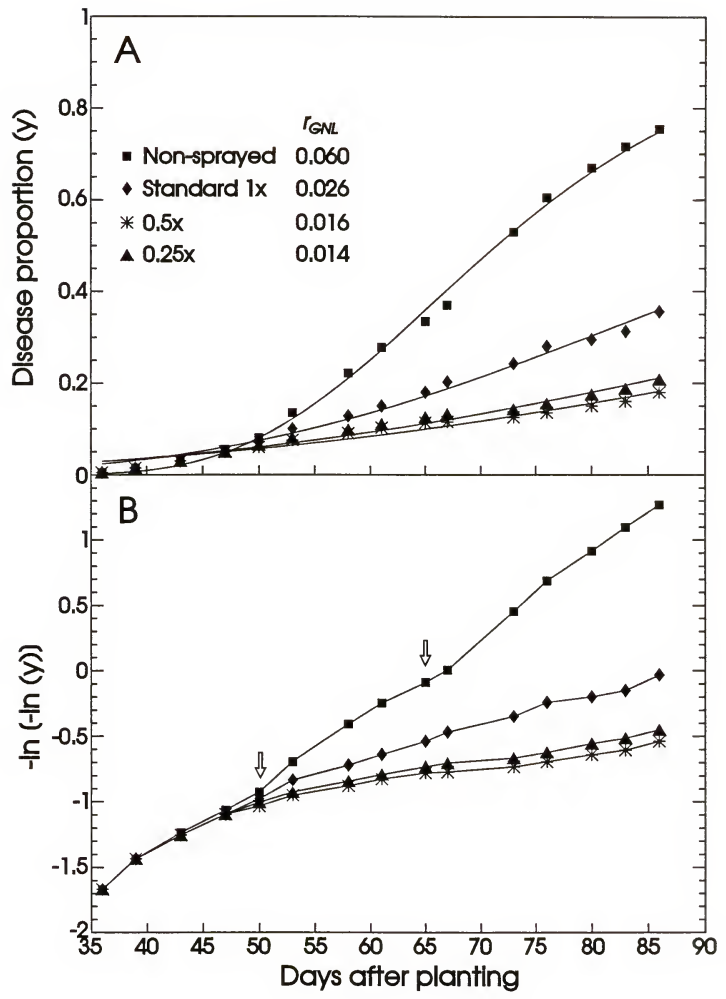


program (Tables 5.5 and 5.6). The mixed schedules I and II were as efficacious on control of disease as sprays of the $0.25 \times$ rate alone (Figs. 5.27 and 5.28). Similar control of disease was also observed between mixed III and IV and season-long sprays of the $0.5 \times$ rate (Figs. 5.29 and 5.30). Among all mixed-spray programs, mixed I and II were significantly $(P=0.05)$ better than mixed III but not different from mixed IV.

Applications of fungicide increased the peanut yield 136 (standard $1 \times$ ) to $166 \%$ (mixed II) over the nonsprayed control in 1997 (Table 5.5). Most of this increase in yield was associated with the control of the late leaf spot, which was the prevailing disease $y_{f}=$ 0.78 ) in 1997. Low variation in yield was observed among spray programs and only the systemic fungicide and the mixed II schedule differed significantly from the standard (1x) treatment.

\section{Relationship of rust and leaf spots to peanut yield}

In all three growing seasons, the pod yield of peanuts was quantified to determine the effects of rust and leaf spots on yield. In 1995 and 1996, the relationship of disease to yield was represented by negative power functions, in which the AUDPC explained $88-93 \%$ the variation in pod yield (Figs. 5.31-5.35). The mixed epidemic of rust and leaf spots in 1995 began only 56 days after planting and resulted in a range in yield (1342 to $2572 \mathrm{~kg} \mathrm{ha}^{-1}$ ) lower than that quantified in 1996 (964 to $2841 \mathrm{~kg} \mathrm{ha}^{-1}$ ), when plants were exposed to the same diseases 12 days earlier (day 44). Peanut rust was the prevailing disease in the experiment established in field not planted to peanut before. Although the rust epidemic began 52 days after planting, the range in yield was as high as $2088 \mathrm{~kg} \mathrm{ha}^{-1}$ (1341 to 3429). Nonsprayed plants of the cultivar Southern Runner yielded twice as much $\left(2094 \mathrm{~kg} \mathrm{ha}^{-1}\right)$ than Florunner $\left(964 \mathrm{~kg} \mathrm{ha}^{-1}\right)$ when planted in the same field 
Fig. 5.27. Disease progress curves (A) and disease waves (B) for mixed epidemics of rust and leaf spots on Florunner peanuts under single- and mixed-rate programs of fungicidal sprays in 1997. The standard spray program for peanuts (1× chlorothalonil) was applied at $35,49,63,77$, and 88 days after planting. The $0.25 \times$ rate was sprayed 15 times (twiceweekly) from day 35 to day 88 . The mixed-spray program I had six twice-weekly sprays of the $0.25 \times$ rate from day 35 to day 53 plus three applications of the $1 \times$ rate on days 57 , 73, and 84. Mixed II comprised eight twice-weekly applications of the $0.25 \times$ rate between days 35 and 60 and three sprays of the full rate on days 63,77 , and 88 . Experiment developed in field planted to peanuts the previous year. The epidemic rates $\left(r_{G N L}\right)$ were estimated by nonlinear regression of disease proportions against time using the Gompertz model. Arrows indicate the ends of epidemic waves. 


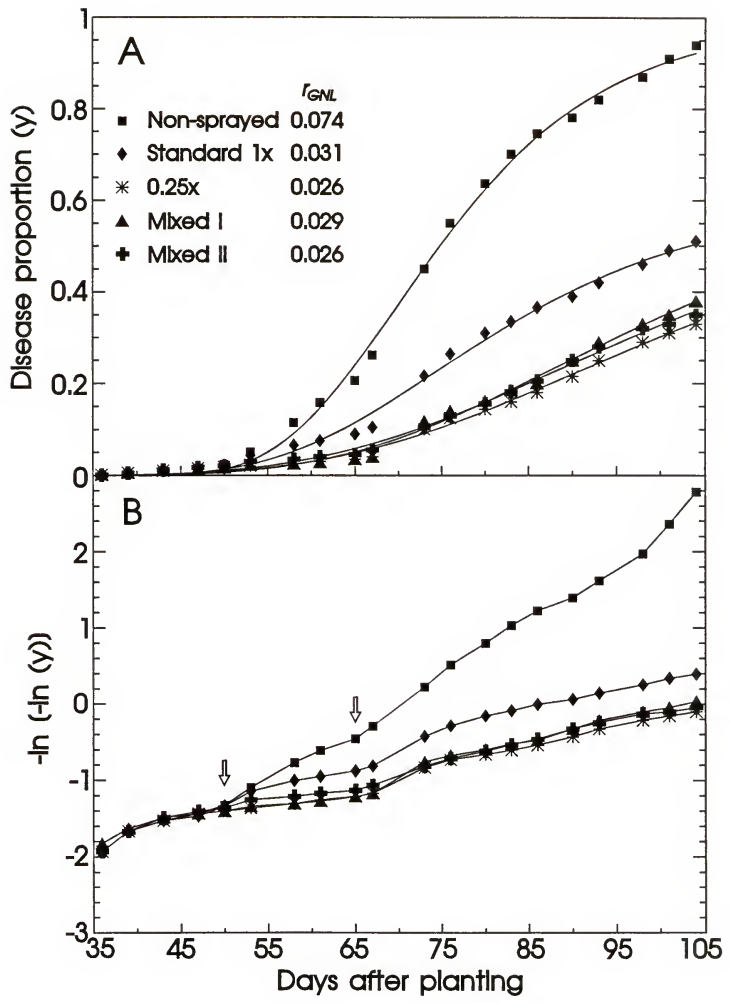


Fig. 5.28. Disease progress curves (A) and disease waves (B) for mixed epidemics of rust and leaf spots on Florunner peanuts under single- and mixed-rate programs of fungicidal sprays in 1997. The standard spray program for peanuts $(1 \times$ chlorothalonil) was applied at $35,49,63$, and 77 days after planting. The $0.25 \times$ rate was sprayed 12 times (twiceweekly) from day 35 to day 75 . The mixed-spray program I had six twice-weekly sprays of the $0.25 \times$ rate from day 35 to day 53 plus two applications of the $1 \times$ rate on days 57 and 73. Mixed II comprised eight twice-weekly applications of the $0.25 \times$ rate between days 35 and 60 and two sprays of the full rate on days 63 and 77. Experiment developed in field planted to peanuts the previous 2 years. The epidemic rates $\left(r_{G N L}\right)$ were estimated by nonlinear regression of disease proportions against time using the Gompertz model. Arrows indicate the ends of epidemic waves. 


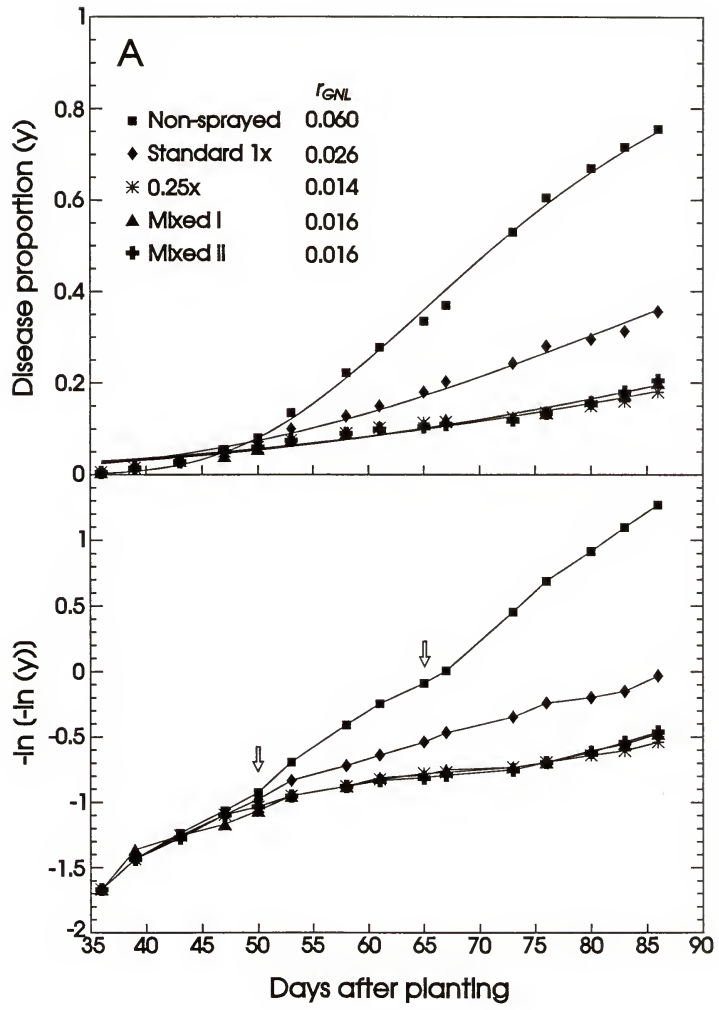


Fig. 5.29. Disease progress curves (A) and disease waves (B) for mixed epidemics of rust and leaf spots on Florunner peanuts under single- and mixed-rate programs of fungicidal sprays in 1997. The standard spray program for peanuts ( $1 \times$ chlorothalonil) was applied at $35,49,63,77$, and 88 days after planting. The $0.5 \times$ rate was sprayed eight times (weekly) from day 35 to day 88 . The mixed-spray program III had three weekly sprays of the $0.5 \times$ rate from day 35 to day 50 plus three applications of the $1 \times$ rate on days 57,73 , and 84 . Mixed IV comprised four weekly applications of the $0.5 \times$ rate between days 35 and 57 and three sprays of the full rate on days 63, 77, and 88. Experiment developed in field planted to peanuts the previous year. The epidemic rates $\left(r_{G N L}\right)$ were estimated by nonlinear regression of disease proportions against time using the Gompertz model. Arrows indicate the ends of epidemic waves. 


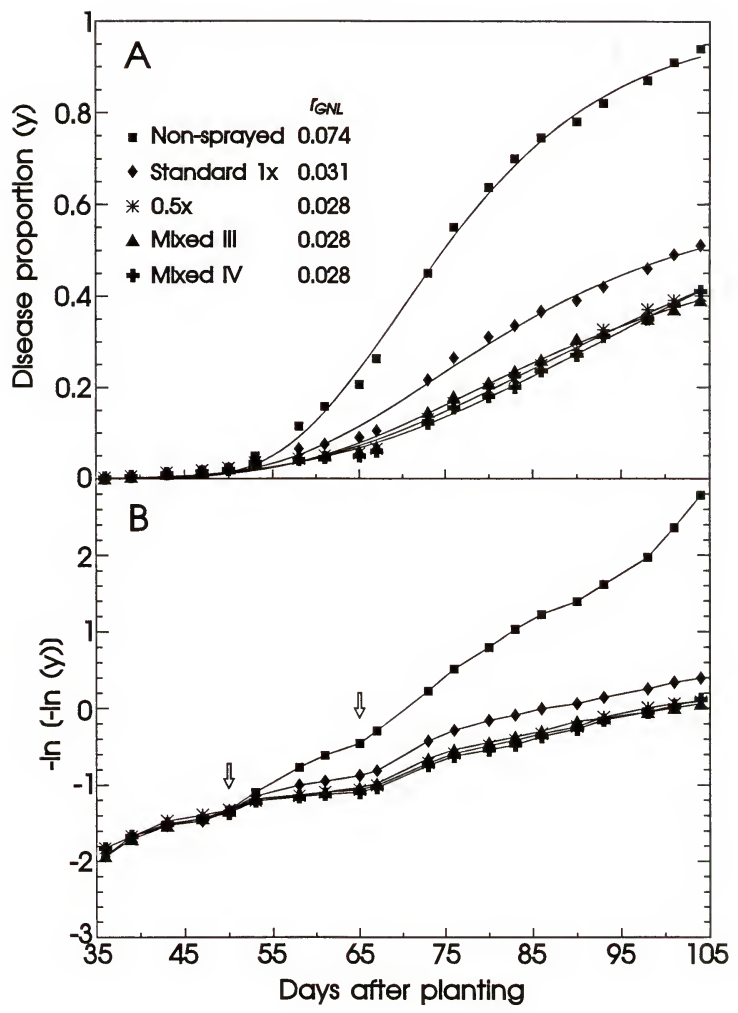


Fig. 5.30. Disease progress curves (A) and disease waves (B) for mixed epidemics of rust and leaf spots on Florunner peanuts under single- and mixed-rate programs of fungicidal sprays in 1997. The standard spray program for peanuts ( $1 \times$ chlorothalonil) was applied at $35,49,63$, and 77 days after planting. The $0.5 \times$ rate was sprayed six times (weekly) from day 35 to day 71 . The mixed-spray program III had three weekly sprays of the $0.5 \times$ rate from day 35 to day 50 plus two applications of the $1 \times$ rate on days 57 and 73 . Mixed IV comprised four weekly applications of the $0.5 \times$ rate between days 35 and 57 and two sprays of the full rate on days 63 and 77 . Experiment developed in field planted to peanuts the previous 2 years. The epidemic rates $\left(r_{G N L}\right)$ were estimated by nonlinear regression of disease proportions against time using the Gompertz model. Arrows indicate the ends of epidemic waves. 


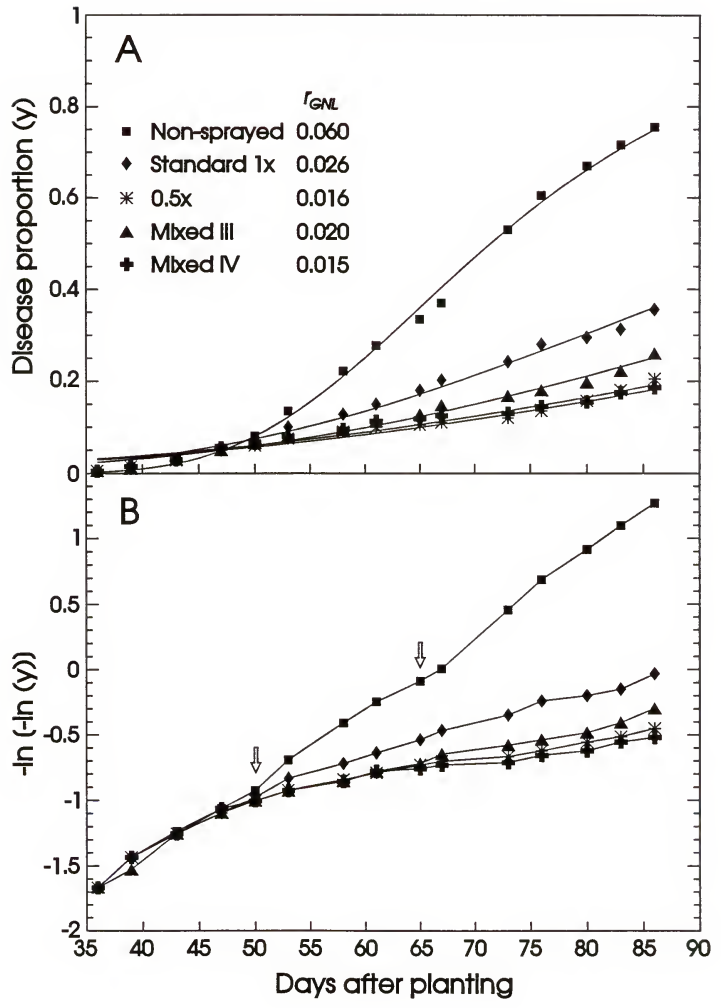




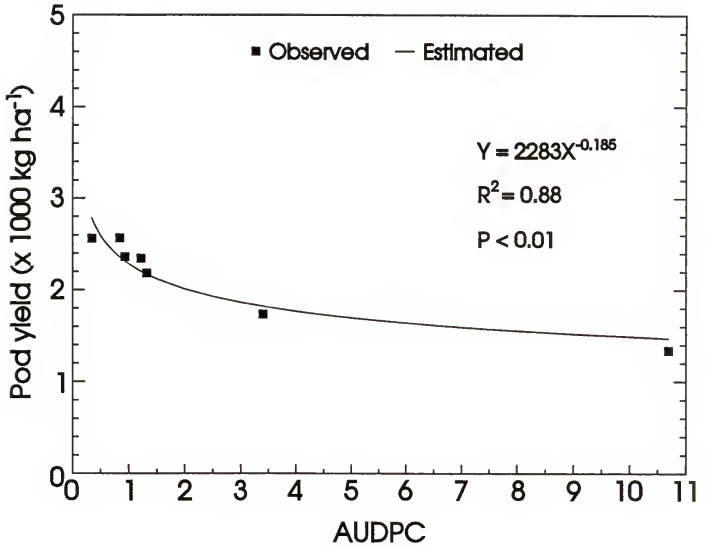

Fig. 5.31. Relationship of area under the disease progress curve (AUDPC) to pod yield by Florunner peanuts associated with mixed epidemics of rust and leaf spots in 1995. The final disease proportion $\left(y_{f}=0.7\right)$ was partitioned into 0.07 for late leaf spot, 0.17 for early leaf spot, and 0.46 for rust. In the negative power function $\mathrm{Y}=2283 \mathrm{X}^{-0.185}, \mathrm{Y}$ is $\mathrm{kg}$ $\mathrm{ha}^{-1}$ and $\mathrm{X}$ is AUDPC. 


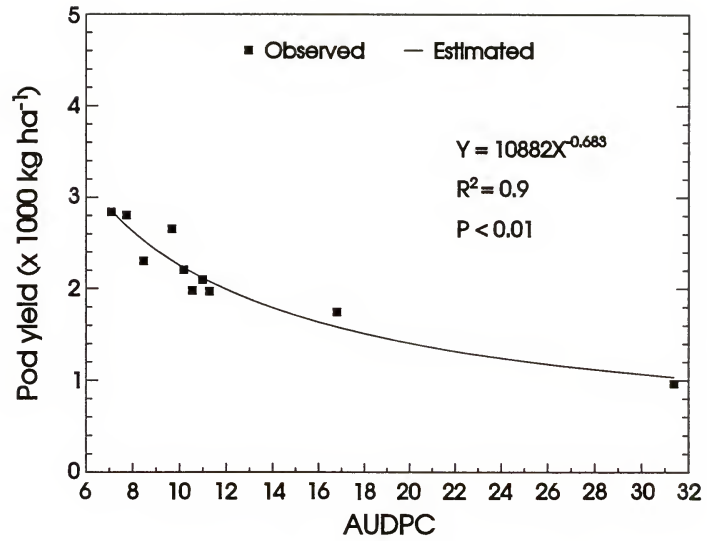

Fig. 5.32. Relationship of area under the disease progress curve (AUDPC) to pod yield by Florunner peanuts associated with mixed epidemics of rust and leaf spots in 1996. The final disease proportion $\left(y_{f}=0.92\right)$ was partitioned into 0.08 for early leaf spot, 0.25 for rust, and 0.59 for late leaf spot. In the negative power function $Y=10882 \mathrm{X}^{-0.683}, \mathrm{Y}$ is $\mathrm{kg}$ $\mathrm{ha}^{-1}$ and $\mathrm{X}$ is AUDPC. 


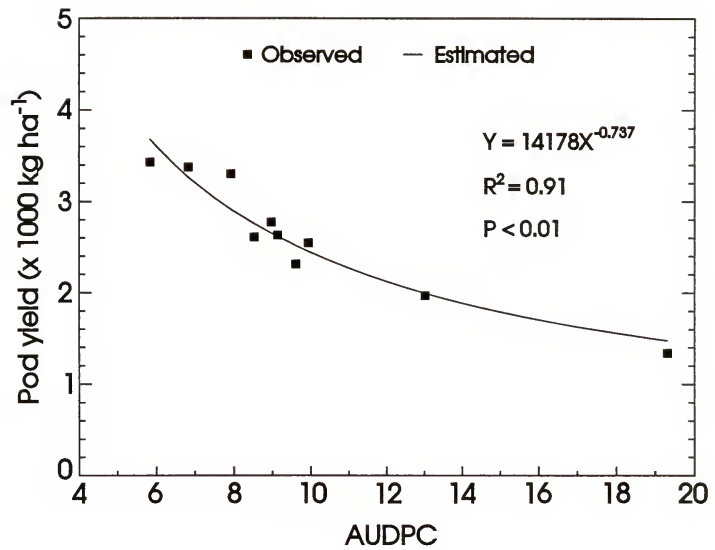

Fig. 5.33. Relationship of area under the disease progress curve (AUDPC) of rust to pod yield of Florunner peanuts in 1996. In the negative power function $\mathrm{Y}=14178 \mathrm{X}^{-0.737}, \mathrm{Y}$ is $\mathrm{kg} \mathrm{ha}^{-1}$ and $\mathrm{X}$ is AUDPC. 


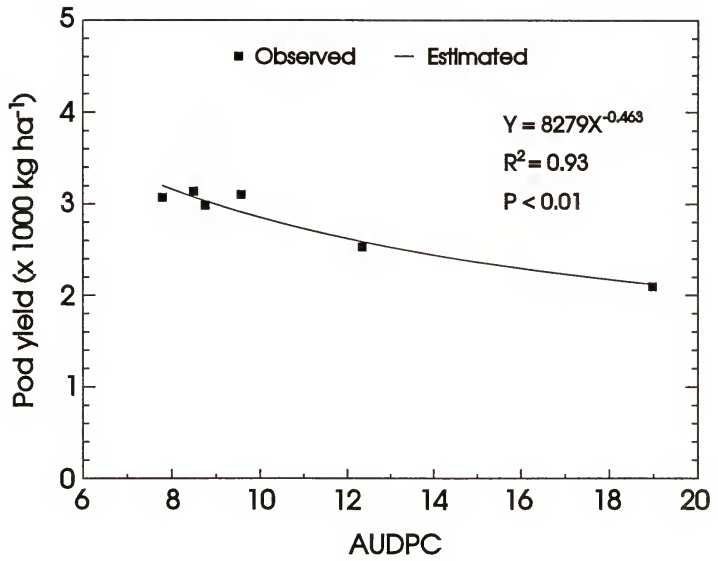

Fig. 5.34. Relationship of area under the disease progress curve (AUDPC) to pod yield by Southern Runner peanuts associated with mixed epidemics of rust and leaf spots in 1996. The final disease proportion $\left(y_{f}=0.64\right)$ was partitioned into 0.32 for late leaf spot and 0.32 for rust. In the negative power function $\mathrm{Y}=8279 \mathrm{X}^{-0.463}, \mathrm{Y}$ is $\mathrm{kg} \mathrm{ha}^{-1}$ and $\mathrm{X}$ is AUDPC. 


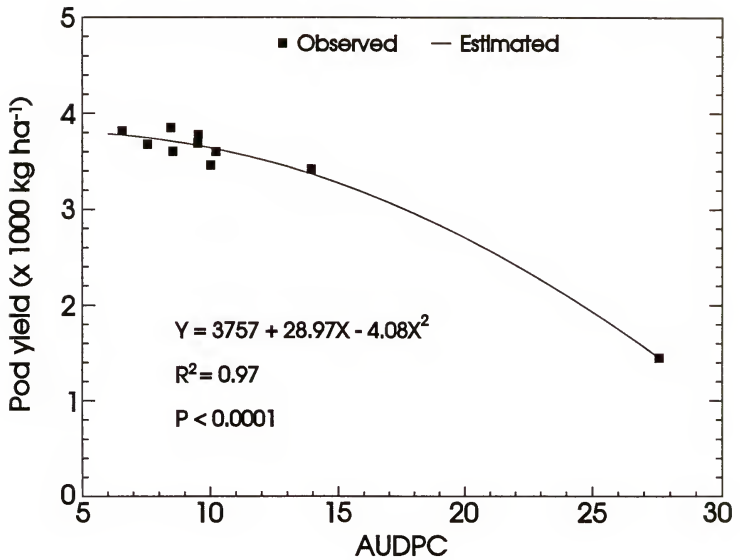

Fig. 5.35. Relationship of area under the disease progress curve (AUDPC) of mixed epidemics of rust and leaf spots to pod yield by Florunner peanuts in 1997. Experiment conducted in field planted to peanuts the previous year. 
and exposed to the same diseases. The range in yield associated with rust and leaf spots was lower in Southern Runner (2094 to $3071 \mathrm{~kg} \mathrm{ha}^{-1}$ ) than in Florunner (964 to $2841 \mathrm{~kg}$ $\left.\mathrm{ha}^{-1}\right)$. In 1997, the late leaf spot was very severe and caused significant yield reduction of the peanut crop. The difference in pod yield between sprayed and nonsprayed plants was as high as $2403 \mathrm{~kg} \mathrm{ha}^{-1}$. In this experiment, the quadratic equation $Y=3757+28.97 X-$ $4.08 X^{2}, R^{2}=0.97$, best fit the relationship of AUDPC to yield (Fig. 5.35).

\section{Discussion}

Although both rust and leaf spots occurred in all six trials on peanuts, the importance of each disease varied depending upon growing season, time of infection, and cultural procedures used in each experiment. Peanut rust was the prevailing disease in 1995 and in one experiment in 1996. The proportion of this disease at the end of the growing seasons ranged from 0.09 to 0.91 . Kucharek (1979) reported that peanut rust occurs sporadically in Florida and usually no earlier than August. Nutter and Shokes (1995) mentioned that rust epidemics might reduce yield $40-70 \%$ if the infection occurs early in the season. In 1995 and 1996, inoculation of plants with P. arachidis in early July resulted in severe epidemics of rust and a yield reduction of $61 \%$. In 1997, the disease occurred naturally from early August and reached a maximum proportion lower than 0.16 . Therefore, the time at which peanut rust infected plants markedly affected the importance of this disease on yield.

Leaf spots, especially late leaf spot, were the major diseases in three of the six field experiments on peanuts. The final proportions of leaf spots ranged from 0.05 to 0.78. Leaf spots increased as peanuts were grown successively in the same areas. For example, the proportion of leaf spots varied from 0.24 in 1995 to 0.67 in 1996 and to 0.73 
in 1997. Although early leaf spot was the major leaf spot in 1995, this disease was rarely seen in 1996 and 1997. In contrast, the late leaf spot increased from 0.07 in 1995 to 0.78 in 1997. The predominance of one disease over the other can shift in any given year or over years in response to changes in climate (Brenneman and Culbreath, 1994; Nutter and Shokes, 1995). However, the increase of the late leaf spot in the experimental area could be explained by the survival ability of $C$. personatum on plant debris. According to Dr. Fred Shokes (personal communication to the author), C. personatum also colonizes the plant stem tissue, which takes long to degrade once buried in, or left on, the soil surface. This assures the pathogen's survival between cropping seasons.

Nutter and Shokes (1995) reported that epidemics of peanut rust increase faster than epidemics caused by leaf-spotting fungi. In 1996, rust and leaf spots differentially affected two experiments planted at the same time and subjected to similar weather. The epidemic rate of rust was higher $\left(0.114\right.$ gompits day $\left.{ }^{-1}\right)$ than that of leaf spots $(0.088$ gompits day $\left.{ }^{-1}\right)$. Under the same conditions, the latent period $(p)$ and the breadth of the epidemic waves were about 10 days long for rust and 14 days for leaf spots. Because of the shorter latent period and the faster epidemic rate of peanut rust, this disease affected the performance of the traditional fungicidal schedules for peanuts, most of them designed and tested to control leaf spots.

Peanut leaf spots are primarily controlled by protectant fungicides applied on a 10- to 14-day schedule, from 30 to 40 days after planting to 14 days prior to digging (Davis et al., 1993; Jacobi et al., 1995; Kucharek, 1979; Kucharek and Shokes, 1995; Linvil and Drye, 1995; Smith and Littrell, 1980). Recently, systemic fungicides (Brenneman and Murphy, 1991; Brenneman and Culbreath, 1994; Kucharek and Shokes, 1995; Labrinos and Nutter, 1993) and weather-based spray programs (Jacobi and 
Backman, 1995; Jacobi et al., 1995) have also been used to control leaf spots on peanuts. The standard calendar schedule, the AU-Pnuts advisory system, and the systemic fungicide tebuconazole were tested in experiments on peanuts developed from 1995 to 1997.

Averaged over 3 years, the standard calendar schedule reduced the AUDPC $64 \%$ compared to the nonsprayed control. The efficacy of the standard schedule decreased from 89 to $49 \%$ as peanuts were planted in the same area and the amount of primary inoculum for the leaf-spotting fungi increased from 1995 to 1997. Although AU-Pnuts averaged one more fungicidal spray than the standard calendar schedule, both treatments provided similar control of the mixed epidemic of rust and leaf spots in 1995 and 1996. AU-Pnuts was significantly better than the calendar schedule in 1997 , in which the proportions of peanut rust in two experiments were less than 0.09 and less than 0.16 . Two main factors might have affected the performance of AU-Pnuts in the presence of peanut rust. First, this advisory system triggered some fungicidal sprays at intervals longer than the latent period ( $p=10$ days) of rust, which allowed the fungus to infect plants before reapplication of fungicide. Second, the amount of moisture $P$. arachidis required to infect plants was lower than the rainfall considered as a rain event in the $\mathrm{AU}$ Pnuts system.

The systemic fungicide tebuconazole was evaluated in four experiments conducted in 1996 and 1997. In both years, sprays of the systemic fungicide were limited to four applications of 0.531 hectare $^{-1}$ per cropping season to prevent development of resistance (Kucharek and Shokes, 1995). To split fungicidal sprays along the season, tebuconazole was initially sprayed one week later than AU-Pnuts and the standard calendar in 1996. In 1997, however, all spray programs began at the same time. The 
systemic fungicide provided better control of rust and higher pod yield than AU-Pnuts and the standard calendar schedule in 1996. Early application of tebuconazole in 1997 improved the control of leaf spots even more. As demonstrated by Barreto et al. (1996), tebuconazole remains active in peanut leaves for more than 14 days, whereas chlorothalonil lasts at most 10 days on the leaf surface. This long-lasting residual of tebuconazole and its ability to reduce disease development soon after application are the major advantages of this fungicide.

Applications of low rates of fungicide at short spray intervals improved the control of peanut rust and leaf spots significantly. Compared to the standard (1x) spray schedule, plants sprayed with $0.5 \times$ and $0.25 \times$ chlorothalonil averaged 27 to $47 \%$ less disease (AUDPC) and 22 to $34 \%$ more yield. The effects of the low fungicidal rates on the epidemics of rust and leaf spots varied depending upon how early the treatments were initiated. For example, delay of epidemic onset and reduction of the amount of initial disease were obtained when the low rates of chlorothalonil were sprayed prior to disease establishment. Otherwise, the major effect of the low fungicidal rates was to slow the epidemic rate $\left(r_{G N L}\right)$. This was possible because the short spray intervals of the low rate schedules maintained enough fungicide on the leaf surface to protect the new plant tissue and to reduce the disease efficiency of the rust and leaf-spotting fungi.

Delay of the first spray of fungicide in one week markedly affected the efficacy of fungicidal treatments. Averaged over three experiments, the AUDPC was $41 \%$ higher and the pod yield $16 \%$ lower on plants sprayed later. The impact of late initiation of spray applications on disease and yield in the experiment under monoculture was twice as much as that observed under crop rotation. For example, a 7-day delay of the first spray of fungicide increased the AUDPC $39 \%$ (averaged over the $0.25 \times, 0.5 \times$, and $1 \times$ rates) 
over the regular applications under monoculture and $20 \%$ after sanitation. Under the same circumstances, pod yield was reduced 16 and $6 \%$. The lower range of disease and yield associated with delay treatments in the crop rotation experiment was due to the low level of primary inoculum for leaf spots in the area planted to peanuts the first time. The full rate of fungicide was more affected by spray delays than the $0.25 \times$ and $0.5 \times$ rates. Although late applications of fungicide did not protect plants from getting infected, weekly or twice-weekly sprays of the low rates reduced the secondary cycles of disease originated from the first infection. This occurred to a less extent on plants sprayed with $1 \times$ chlorothalonil at every 14 days. Overall, the benefits of delaying epidemic onset and reducing initial disease were lost when fungicidal treatments were initiated after disease establishment.

Because season-long sprays of reduced rates of fungicide may not be economically viable or may be difficult to implement, various mixed-rate schedules were tested in 1996 and 1997. The idea behind the mixed schedules was to replace the first and second sprays of fungicide in the standard $(1 \times)$ calendar schedule with applications of low fungicidal rates at short intervals. Such modification of the standard spray program should improve the control of the early stages of peanut rust and leaf spots. Indeed, splitting of two biweekly sprays of the $1 \times$ rate of chlorothalonil into six to eight applications of the $0.25 \times$ rate reduced the AUDPC $39-40 \%$ over the standard treatment. Under the same conditions, three to four weekly sprays of the $0.5 \times$ rate early in the season lowered the AUDPC 29-33\%. In 1997, the mixed-rate schedules that included the $0.25 \times$ rate did not differ statistically from season-long sprays of $0.25 \times$ chlorothalonil alone. Similar results were also obtained between the $0.5 \times$ rate and its mixed spray 
programs. In 1996, by mistake, sprays of the full rate of chlorothalonil were initiated 14 days (instead of 3 days) after applications of the $0.25 \times$ rate had stopped. This affected the efficacy of the mixed-rate treatment, which was statistically less efficacious than applications of the $0.25 \times$ rate alone. Even in this case, however, plants sprayed according to the mixed schedule had $25 \%$ less disease and $17 \%$ more yield than those subjected to the standard $(1 \times)$ spray program for peanuts.

In 1996, various fungicidal spray programs were tested in experiments developed in two adjacent fields, one planted to peanuts the previous year and the other one fallow. These fields were located side-by-side and subjected to similar cultural practices. The area not planted to peanuts before was used to evaluate the interaction between sanitation (by crop rotation or fallow) and fungicidal spray programs on epidemics of peanut leaf spots. The area maintained without cropping was probably not free of inoculum, since dispersal of fungal propagules might have occurred during land preparation or by wind. Despite this fact, the first symptoms of leaf spots were detected at least 9 days later in the experiment under sanitation than in the area under monoculture of peanuts. Also, the progress of leaf spots in the field not planted to peanuts before was very slow and resulted in a proportion of final disease of only 0.05 . In contrast, peanut rust accounted for 0.91 of the final disease proportion $\left(y_{f}=0.96\right)$ in this experiment.

Because of absence of visual symptoms on plants grown in the area not planted to peanuts before, the first sprays of fungicide were carried out 45 days after planting, that is, 7 days later than in the other experiment. While the low rates $(0.25 \times$ and $0.5 \times)$ of fungicide only slowed the epidemic rate $\left(r_{G N}\right)$ in the experiment under monoculture, significant reduction of initial disease $(0.5 \times$ rate) and delay of epidemic onset $(0.25 \times)$ 
were obtained in the area subjected to sanitation (fallow). Therefore, sanitation improved the management of peanut leaf spots three ways: $i$, it delayed occurrence of the diseases; $i i$, allowed the postponement of the first fungicidal spray, and $i i i$, improved the efficacy of fungicidal treatments.

The peanut cultivar Southern Runner, which has partial resistance to leaf spots (Gorbet et al., 1986; Watson, 1987), was included in one experiment developed in 1996. Peanut plants were grown in area with inoculum of the leaf-spotting fungi from the previous year and were also inoculated with $P$. arachidis. At the end of the cropping season, the nonsprayed plants had similar amounts of rust (0.32) and late leaf spot $(0.3)$, and very low proportion of the early leaf spot $(0.02)$. The epidemic rate for this disease complex $\left(0.077\right.$ gompits day $\left.{ }^{-1}\right)$ was lower than that observed for the cultivar Florunner $\left(0.088\right.$ gompits day $\left.{ }^{-1}\right)$ in an experiment developed side-by-side. The average latent period of peanut rust was 2 days longer in Southern Runner plants (12 days) than in Florunner (10 days). Therefore, the cultivar Southern Runner also showed partial resistance to peanut rust.

The rate-reducing resistance of Southern Runner has allowed the fungicides to be sprayed at long intervals and to decrease the total fungicide required for disease control (Culbreath et al., 1992; Jacobi and Backman, 1995; Kucharek et al., 1991; Kucharek and Shokes, 1995; Maytac and Bailey, 1988). In the 1996 experiment on Southern Runner, various fungicidal schedules were tested at spray intervals longer than those used to control rust and leaf spots on Florunner peanuts. With the spray interval lengthened from 14 to 21 days, the efficacy of the standard $(1 \times)$ treatment was reduced. This lower fungicidal efficacy might have resulted from the presence of infected plants prior to initiation of spray applications and from the simultaneous occurrence of peanut rust. 
Applications of fungicide according to the low-rate schedules reduced AUDPC 55\% ( $0.5 \times$ rate $)$ to $59 \%(0.25 \times$ rate $)$ and increased yield $47 \%(0.25 \times$ rate $)$ to $50 \%(0.5 \times$ rate $)$. Fry (1975 and 1978) had already reported that either the reduction of the fungicidal rate or the lengthening of spray interval could be combined with polygenic resistance to control late blight on potato. In this research on peanuts, the rate-reducing resistance of the cultivar Southern Runner allowed low rates and long spray intervals to be used together to control disease.

The relationship of disease to peanut yield was represented by a quadratic equation and negative power functions, in which the AUDPC explained $88-97 \%$ the variation in pod yield. Reductions in yield were higher as the epidemics of rust and leaf spots began earlier. In Florunner peanuts, rust caused more yield losses than leaf spots in two of the tree cropping seasons. Because its partial resistance to rust and leaf spots, the cultivar Southern Runner was less affected by the disease complex and yielded twice as much as Florunner. 


\section{CHAPTER VI \\ MANAGEMENT OF EPIDEMICS OF BLACK SPOT ON ROSES}

\section{Introduction}

Black spot, caused by Diplocarpon rosae, is the most important disease of roses worldwide (Horst, 1986; Leahy, 1990). In the southeastern U.S., roses are often devastated by this disease (Bowen et al., 1995), especially when plants are grown under outdoor ambient conditions (Horst, 1986; Leahy, 1990). Infected leaves develop chlorosis and abscise prematurely, which results in low-vigor plants and reduced production of flowers.

Because resistance in roses to black spot is rare, the control of this disease relies on sanitation, management of irrigation, and largely on applications of fungicides (Bowen et al., 1995; Horst, 1986; Leahy, 1990). Horst (1986) mentioned that, in the northeastern U.S., preventive sprays of fungicide should be initiated around February, repeated bimonthly until leaf emergence, and then continued at weekly intervals. Bowen et al. (1995) observed that weekly applications of fungicides were neither desirable nor convenient for homeowners. Horst (1986) and Bowen et al. (1995), however, recognized that chemical control of black spot was possible only if regular and frequent applications of protectant fungicides were made throughout the growing season.

Under the warmer and wetter weather conditions of the southeast U.S., epidemics of black spot may be fast and severe. For instance, at temperatures of $22-30^{\circ} \mathrm{C}$, the incubation period for black spot can be as short as 3-4 days (Horst, 1986). Additionally, this disease is more severe on young leaves which are produced abundantly during the 
rose-growing season in Florida (McFadden and Conover, 1975). New leaves may also develop during the time interval between fungicide treatments. Under conditions of rapid leaf production, black spot is less likely to be controlled by fungicidal sprays applied at long intervals.

Because of the economic importance of the rose industry in Florida, the losses attributed to black spot, and the importance of fungicides for the control of this disease, this pathosystem was included in this investigation on the management of epidemics with reduced rates of fungicides. Several combinations of rates and spray intervals for fungicide applications were evaluated to reduce initial disease, to delay epidemic onset, and to slow the epidemic rate. This investigation was conducted during summer, fall, and spring conditions, so that disease control was evaluated under a wide range of different environmental situations.

\section{Material and Methods}

Three experiments were conducted successively during the Summer and Fall of 1996 and Spring of 1997, in Gainesville, FL. Individual rose plants of varieties Dolores, John F. Kennedy (JFK), and Oregold, kindly provided by Dewar Nurseries Inc. (Apopka, FL 32703), were grown in 11.4-1 pots kept outdoors under black, $46 \%$-shade cloth. The roses were fertilized every 2 weeks with a 20-20-20 (N-P-K) soluble fertilizer (Peters Professional, Grace-Sierra Horticultural Products Co., Milpitas, CA) at the rate of $5 \mathrm{~g}$ per plant. Water was applied directly to the soil surface daily unless rain occurred. Plants were arranged in a randomized complete block design with seven treatments and three replications, each variety being a replication. The distance between plants within and between blocks was $1 \mathrm{~m}$. The fungicides mancozeb (Manzate $200 \mathrm{DF}$ ) and cupric hydroxide (Kocide 101, Griffin Corporation, Valdosta, GA), recommended for the 
control of major rose diseases (Simone et al., 1994), were applied according to various spray schedules as described below for each treatment. Fungicidal rates were adjusted in terms of milligrams of the active ingredients per 11 of water and sprayed to run-off. Fungicides were applied by means of an 1.51 , hand-held sprayer (Delta Industries, King of Prussia, PA) bearing an $80^{\circ}$ cone nozzle. An average of $150 \mathrm{ml}$ of material was delivered to each plant at each spray, and no adjuvants were added to the fungicidal suspensions. Disease severity was assessed twice a week by visual estimation of the proportion of disease and corrected for defoliation with the formula presented earlier for peanuts (Plaut and Berger, 1980; Shokes et al., 1987). Defoliation was determined based on the proportion of stems without leaves. Lesion expansion ( $\mathrm{mm}$ ) was evaluated in the fall experiment in 1996. Young lesions of black spot were marked in seven leaves of Dolores and five leaves of JFK and assessed for 16 consecutive days. The rate of lesion expansion $(R L E)$ was calculated as $R L E=\left(L D_{f}-L D_{i}\right) / \Delta t$, in which $L D_{i}$ and $L D_{f}$ are initial and final lesion diameters, and $\Delta t$ is the time interval between $L D_{i}$ and $L D_{f}$. Flower production was quantified during the fall and spring experiments by counting flowers per plant at each pruning. Only the tops of the flower-bearing stems were pruned, so that all leaves were preserved for disease assessment.

\section{$\underline{\text { Summer Experiment }}$}

The summer experiment on roses was developed from May to July 1996. To eliminate both symptomatic and asymptomatic infected plant tissue, plants were scouted for disease for 10 days and all diseased leaves were removed from the plants. Fungicidal sprays were then initiated on 1 May 1996 and repeated weekly for $1 \times$ mancozeb $(225 \mathrm{~g}$

a.i. $\left.100 \mathrm{r}^{-1}\right)$ and $1 \times$ cupric hydroxide $\left(231 \mathrm{~g}\right.$ a.i. $\left.100 \mathrm{l}^{-1}\right)$, and semiweekly for $0.25 \times$ 
mancozeb and $0.25 \times$ cupric hydroxide. Two other treatments, both of which included the fungicide chlorothalonil, caused severe phytotoxicity and were subsequently replaced with mixtures of $0.25 \times$ mancozeb plus $0.25 \times$ cupric hydroxide, and $0.1 \times$ mancozeb plus $0.1 \times$ cupric hydroxide. These mixtures were applied to plants at weekly $(0.25 x+0.25 x)$ and semi-weekly intervals $(0.1 \times+0.1 \times)$, respectively. Diplocarpon rosae was inoculated on 2 May 1996 and again on 6 May 1996 on one stem of each plant. The inoculum was prepared by washing diseased leaves in distilled water that contained two drops of Tween 20 per $100 \mathrm{ml}$ of water. Inoculations were made between 19:00 and 20:00 hrs and the inoculated stems remained covered with plastic bags for 12-14 hrs. The statistical analyses of the epidemic parameters $t_{0}, y_{0}, y_{\max }, r_{G N L}$, and AUDPC for black spot on roses followed the same procedures previously described for experiments on oats.

\section{Fall Experiment}

The fall experiment was conducted in September and October of 1996. On 15 August 1996, the stems were pruned to a height of $15-20 \mathrm{~cm}$ and all remaining leaves and other plant debris were removed. New fungicide schedules were then initiated on 3 September 1996. The fungicides mancozeb and cupric hydroxide were applied to plants at $1 \times, 0.5 \times$, and $0.25 \times$ of their label rates. The treatments were repeated weekly for the full rate $(1 \times)$ and semi-weekly for the reduced doses $(0.5 \times$ and $0.25 \times)$. On 15 September 1996, one stem of each plant was inoculated with a spore suspension of $D$. rosae as described above in the summer experiment. Insecticides were applied twice to control aphids and other insects. 


\section{Spring Experiment}

The spring experiment was developed in February-April 1997 under the same conditions described for the previous experiments. All stems were pruned at $15-20 \mathrm{~cm}$ height on 10 February 1997. Fungicidal applications were initiated on 15 February 1997 with the same fungicides, rates, and time intervals tested in the fall experiment. On 7 March 1997, the rose plants were inoculated with a spore suspension of $D$. rosae applied to the whole plant. Disease was assessed at 2- to 3-day intervals between 15 March 1997 and 7 April 1997.

\section{$\underline{\text { Results }}$}

\section{Characteristics of Black Spot Epidemics}

Experiments on management of black spot on roses in three growing seasons resulted in epidemics with different characteristics (Fig. 6.1). In summer, the epidemic began 10 days after inoculation and reached its maximum disease severity $\left(y_{\max }=0.897\right)$ 51 days later. The epidemic rate $\left(r_{G N L}\right)$ was 0.105 gompits day ${ }^{-1}$. In fall, the black spot epidemic began earlier (eight days after inoculation), developed at a faster epidemic rate ( 0.239 gompits day $\left.{ }^{-1}\right)$, and was shorter in duration ( 29 days) than in summer. The maximum disease proportion was 0.733 . The increase of disease after 29 days was limited by cooler temperatures recorded 3 weeks after inoculation (Appendix B). The first symptoms of black spot in the spring experiment were observed 8 days after inoculation. The disease severity increased from 0.005 to 0.94 in only 23 days. The epidemic rate of black spot in this experiment was 0.206 gompits day $^{-1}$.

The estimated daily increments of disease severity $\left(d_{y} / d_{t}\right)$ (Fig. 6.2) were higher in fall and spring than in summer. In the latter growing season, the maximum $d_{y} / d_{t}$ was 0.04 at 30 days after inoculation ( 18 days after appearance of the first symptoms). Forty 


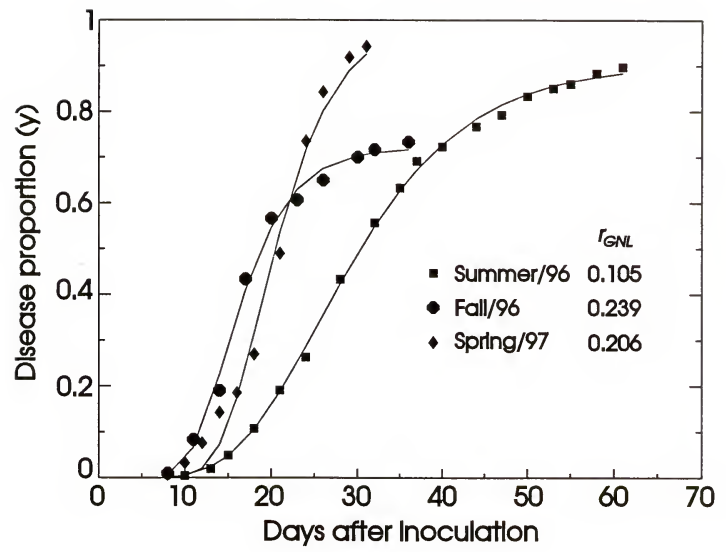

Figure 6.1. Disease progress curves for black spot of rose on nonsprayed plants in Summer and Fall 1996, and Spring 1997. Epidemic rates $\left(r_{G N L}\right)$ were estimated by nonlinear regression of disease proportions against time using the Gompertz model. 


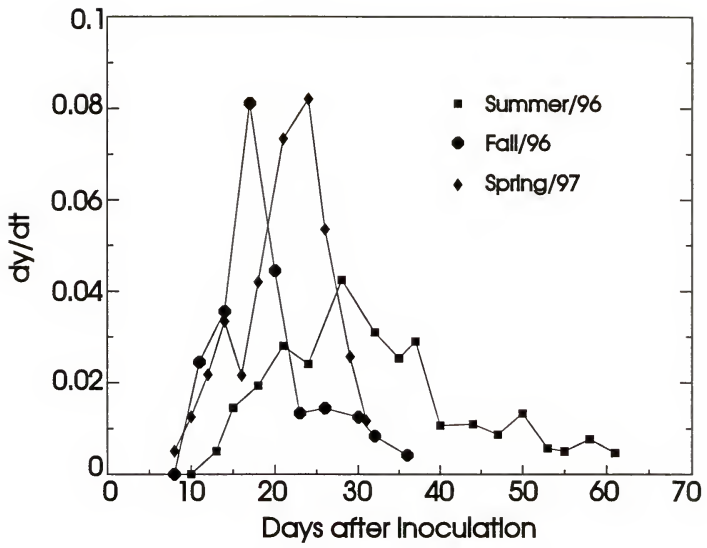

Figure 6.2. Estimated daily increment of disease severity $\left(d_{d} / d_{t}\right)$ for epidemics of black spot of roses on nonsprayed plants. Experiments conducted in Summer and Fall 1996, and Spring 1997. 
days after inoculation, $d_{y} / d_{t}$ decreased gradually as the availability of host tissue was lowered. In the fall experiment, $d_{y} / d_{t}$ was as high as 0.08 at 17 days after inoculation. In the spring experiment, the maximum $d_{y} / d_{t}$ was 0.08 at 25 days. After these peaks of disease increase, $d_{y} / d_{t}$ was greatly reduced because of low availability of host tissue and low temperatures in the fall experiment.

The incubation and latent periods of black spot varied among rose cultivars and growing seasons. In summer, the incubation period was 10 days for Dolores and Oregold and 12 days for JFK. The latent period was 4 days longer than the incubation period for all cultivars, that is, it ranged from 14 to 16 days. In fall, the incubation period ranged from 6 days (Dolores and Oregold) to 8 days (JFK), whereas the latent period was 8 days (Dolores and Oregold) to 10 days (JFK). In spring, the average incubation period was 7 days and the average latent period was 8 days.,

In addition to development of new lesions, lesion expansion and defoliation were also major components of the total disease severity. Although the average rate of lesion expansion (RLE), that is, increase in diameter, was $0.34 \mathrm{~mm}^{-19}{ }^{-1}$ for $\mathrm{JFK}$ and $0.75 \mathrm{~mm}$ day $^{-1}$ for Dolores, RLE was as high as $1.35 \mathrm{~mm} \mathrm{day}^{-1}$ in some leaves. This led to fast and severe defoliation of the rose plants, which occurred from 9 (JFK) to 14 days (Dolores) after the appearance of the first lesions. The variety Oregold had defoliation as severe as that of JFK.

\section{Effects of Fungicidal Spray Programs on Epidemic Onset and Amount of Initial Disease}

In the summer experiment, the first disease symptoms were detected on 12 May 1996, which was considered as epidemic onset $\left(t_{0}\right)$. All plants showed some symptoms on $t_{0}$, so that there was no delay in epidemic onset for any fungicidal treatment. In fall, black spot was initially detected on 23 September 1996 on nonsprayed plants and 
epidemic delays of 7 days were observed on plants treated with $0.25 x$ and $0.5 x$ mancozeb. In spring, the various fungicidal treatments did not affect epidemic onset, which occurred on plants in all treatments on 17 March 1997.

The initial disease severity $\left(y_{0}\right)$ varied statistically among fungicidal treatments (Table 6.1). In summer, the proportion of initial disease was significantly lower $(P=$ 0.05 ) on plants sprayed with mancozeb or mancozeb plus cupric hydroxide. Comparisons of initial disease intensities among rates of fungicide, however, were not significant. Single sprays of cupric hydroxide, even at its full rate, did not reduce $y_{0}$. Initial disease severity measured on the control plants was higher in the fall (0.01) than in the summer experiment (0.005). Even with a higher initial disease intensity, all fungicidal treatments reduced $y_{0}$ significantly $(P=0.05)$. Applications of mancozeb at the $0.25 \times$ or $0.5 \times$ rates provided complete control of the disease. Therefore, these treatments were not included in the statistical comparisons for initial disease shown in Table 6.1. Rates of cupric hydroxide did not differ from each other in reduction of $y_{0}$. In spring, all fungicidal treatments, except $1 \times$ cupric hydroxide, reduced the initial amount of disease significantly $(P=0.05)$. The full and lower rates of mancozeb were equally efficacious in reducing $y_{0}$.

\section{Effects of Fungicidal Spray Programs on the Epidemic Rate}

The epidemic rates for black spot on roses are shown in Table 6.2, in which $r_{G N L}$ refers to epidemic rates estimated by nonlinear regression of disease proportions against time using the Gompertz model. In the summer experiment, black spot progressed at a rate of 0.105 gompits day ${ }^{-1}$ on the control plants. The epidemic rates were significantly slower $(P=0.05)$ on treated plants $\left(0.049\right.$ to 0.061 gompits day $\left.{ }^{-1}\right)$, except for those 


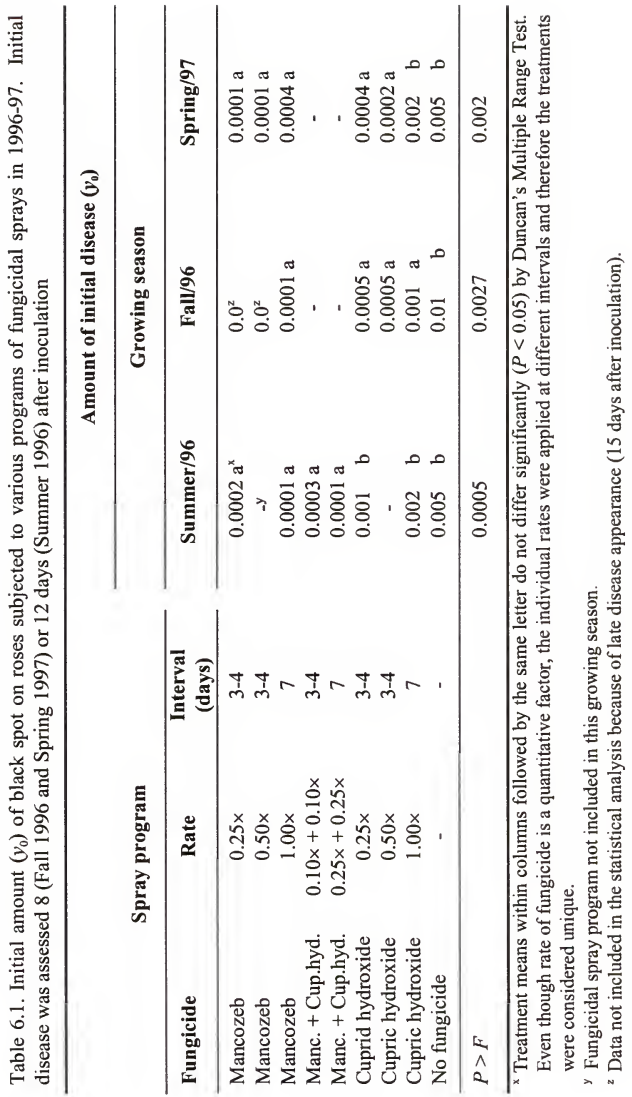




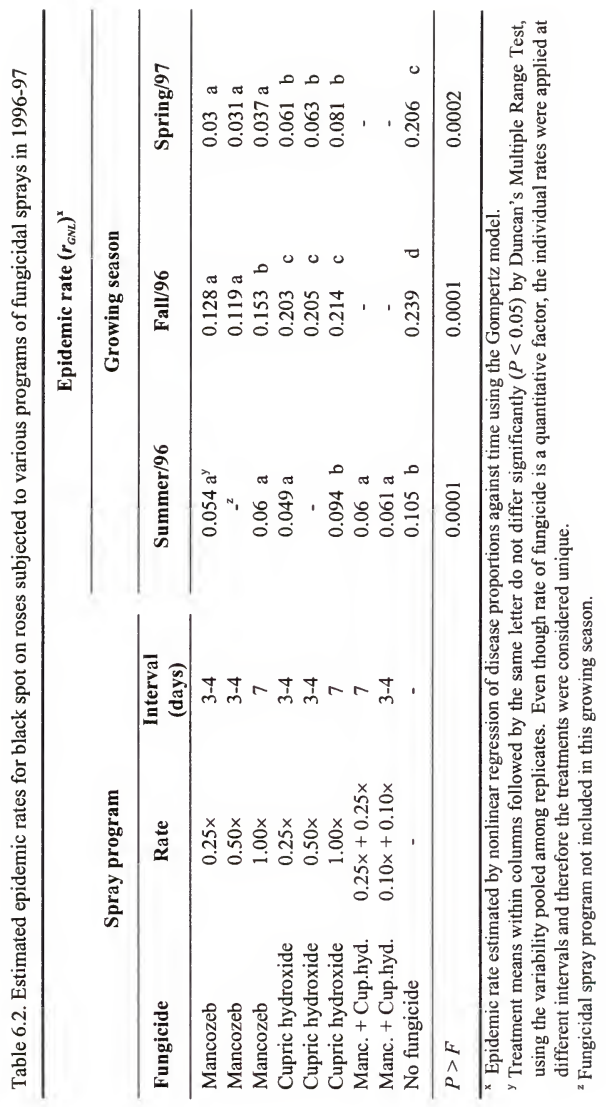


sprayed with $1 \times$ cupric hydroxide, whose epidemic rate did not differ from the nonsprayed control. In the fall experiment, both cupric hydroxide and mancozeb slowed the epidemic progress significantly $(P=0.05)$. Plants treated with mancozeb had slower epidemic rates than plants treated with cupric hydroxide. Sprays of lower fungicidal rates applied twice a week did not affect $r_{G N L}$ on plants treated with cupric hydroxide. In contrast, reduced rates of mancozeb sprayed twice a week significantly $(P=0.05)$ slowed $r_{G N L}$ compared to the full rate sprayed weekly. In spring, $r_{G N L}$ was once again slower for mancozeb than for cupric hydroxide. No differences were observed among rates within each fungicidal treatment applied at their respective intervals.

\section{Effects of Fungicidal Spray Programs on the Amount of Final Disease}

The proportion of final disease $\left(y_{f}\right)$ (Table 6.3) varied among growing seasons and fungicidal treatments. In summer, $y_{f}$ was 0.897 at 53 days after the appearance of the first symptoms on the control plants. All fungicidal treatments reduced $y_{f}$ significantly $(P=$ 0.05). Differences between rates of the same fungicide, applied at their respective intervals, were observed for mancozeb but not for cupric hydroxide. Mixtures of reduced rates of mancozeb and cupric hydroxide, applied twice weekly, had similar $y_{f}$ to that of the full rate of mancozeb applied weekly. In the fall experiment, the epidemic curve of the control treatment reached its $y_{f}(0.733)$ at $t_{29}$. On plants treated with cupric hydroxide, $y_{f}$ ranged from 0.27 to 0.37 for the three rates of application, being significantly lower ( $P$ $=0.05$ ) than that of the control treatment. Mancozeb reduced $y_{f}$ to values as low as 0.003 (for the $0.25 \times$ rate) and differed statistically $(P=0.05)$ from the control and cupric hydroxide treatments. For both mancozeb and cupric hydroxide, however, no differences in $y_{f}$ were attributed to fungicidal rates. Although the epidemic in Spring 1997 was the 


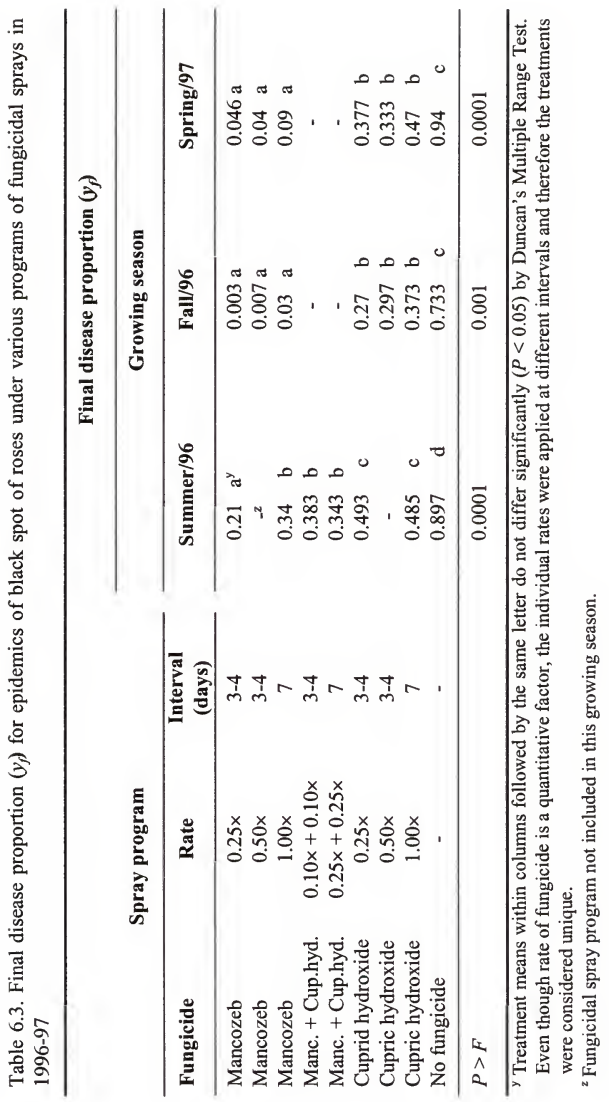


shortest one, the proportion of final disease was the highest (0.94). All fungicidal treatments reduced $y_{f}$ significantly $(P=0.05)$ compared to the control, but, again, no statistical differences were detected among rates within each fungicide.

\section{Effects of Fungicidal Spray Programs on the Epidemic Waves}

Although the disease waves for the various epidemics of black spot on nonsprayed plants were greatly flattened, two waves still could be distinguished for the summer and fall epidemics and one for the spring epidemic (Fig. 6.3). In summer, the first disease wave occurred between days 10 and 25 and the second wave was observed between 25 and 40 days after inoculation. Both epidemic waves lasted about 15 days, which was the average duration of the latent period for black spot in summer. The gompit values at the beginning and the end of the epidemic waves in Summer 1996 were -1.667 and -0.289 for the first wave, and -0.289 and 1.126 for the second wave. On a linear scale black spot progressed from 0.005 to 0.263 during the first wave and from 0.263 to 0.723 during the second wave (Table 6.4).

In the summer all fungicidal treatments reduced the amount of disease at the end of each wave (Table 6.4 and Fig. 6.4-6.6). Sprays of mancozeb or mancozeb plus cupric hydroxide reduced the height of the epidemic waves more efficiently than cupric hydroxide. The lower rate $(0.25 \times)$ of mancozeb sprayed twice weekly had a lower second epidemic wave than the full rate applied weekly. Applications of $0.25 \times$ cupric hydroxide twice a week resulted in lower epidemic waves than sprays of the full rate once a week. The spray programs based on mixtures of mancozeb plus cupric hydroxide had similar wave development and did not differ from each other in disease intensity. 


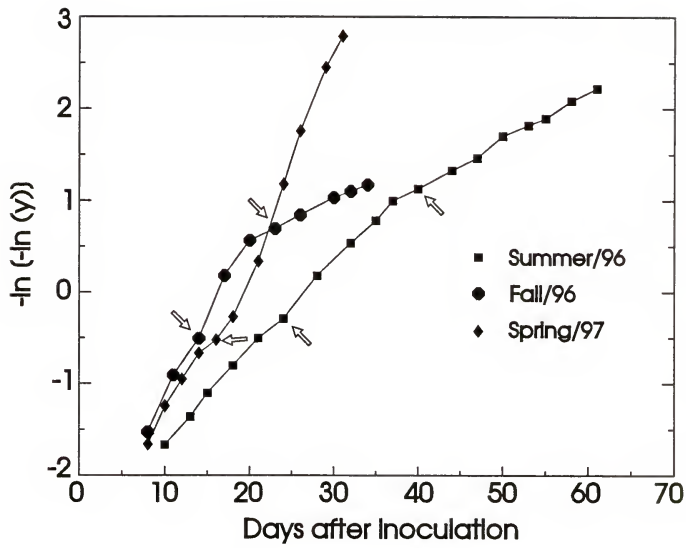

Figure 6.3. Epidemic waves of black spot of roses on nonsprayed plants. Disease proportions $(y)$ were transformed by $-\ln [-\ln (y)]$. Arrows indicate the end of epidemic waves. Experiments developed in Summer and Fall 1996, and Spring 1997. 


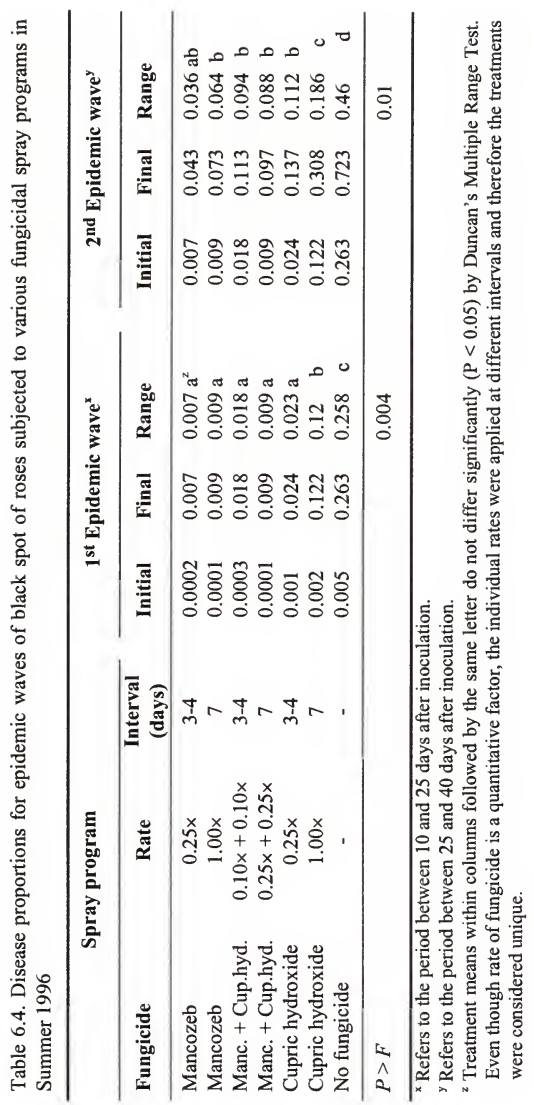




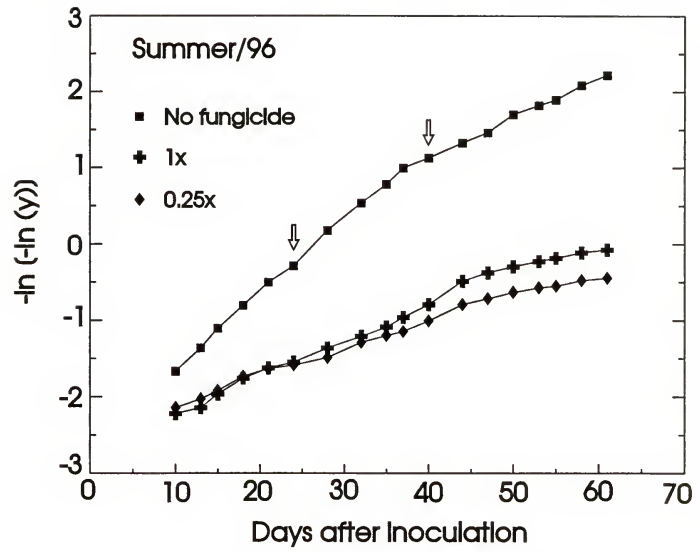

Figure 6.4. Epidemic waves of black spot of roses under weekly (1× rate) or twiceweekly sprays $(0.25 \times$ rate $)$ of the fungicide mancozeb. Disease proportions $(y)$ were transformed by $-\ln [-\ln (y)]$. Arrows indicate the end of epidemic waves. Experiment developed in Summer 1996. 


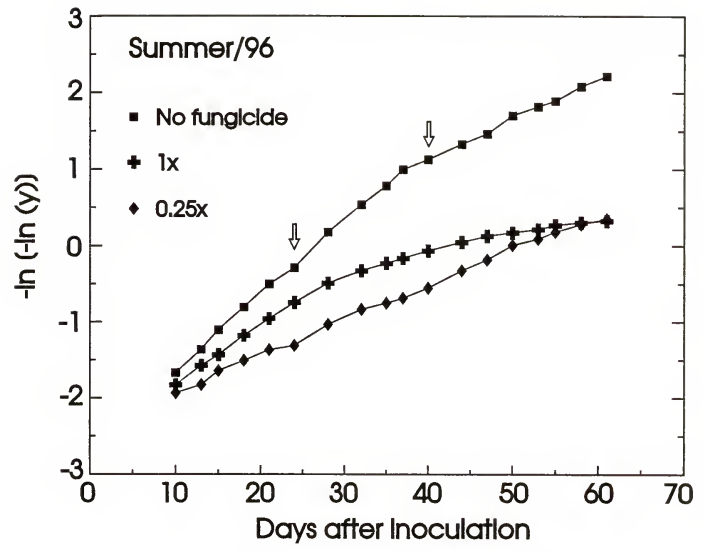

Figure 6.5. Epidemic waves of black spot of roses under weekly (1× rate) or twiceweekly sprays $(0.25 \times$ rate $)$ of the fungicide cupric hydroxide. Disease values $(y)$ were transformed by $-\ln [-\ln (y)]$. Arrows indicate the end of epidemic waves. Experiment developed in Summer 1996. 


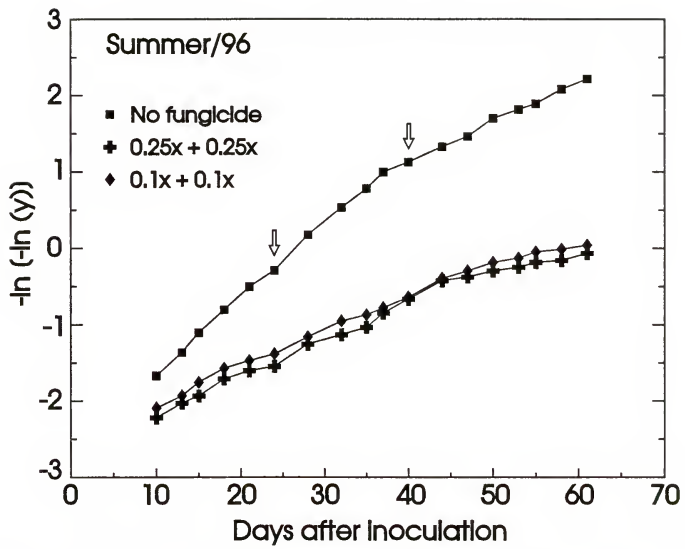

Figure 6.6. Epidemic waves of black spot of roses under weekly $(0.25 x+0.25 x$ rate) or twice-weekly sprays $(0.1 \times+0.1 \times$ rate $)$ of the mixture of mancozeb plus cupric hydroxide. Disease proportions $(y)$ were transformed by $-\ln [-\ln (y)]$. Arrows indicate the end of epidemic waves. Experiment developed in Summer 1996. 
In fall, the first epidemic wave was observed between days 8 and 14 , and the second wave between days 14 and 22 . The average wave length was 7 days. The gompit values ranged from -1.527 to -0.507 for the first, and from -5.507 to 0.694 for the second wave., that is, the variation in disease proportion (Table 6.5) was from 0.01 to 0.19 , and from 0.19 to 0.606 .

In the fall experiment, black spot increased 0.18 and 0.417 during the first and second epidemic waves observed on nonsprayed plants (Table 6.5 and Figs. 6.7-6.8). Although both cupric hydroxide and mancozeb reduced the range of disease increase significantly $(P=0.05)$, mancozeb was far superior. The epidemic waves for the three rates of cupric hydroxide had similar development and did not differ significantly in their disease intensity.

Because of the epidemic delays observed on plants treated with reduced rates $(0.25 \times$ and $0.5 \times)$ of mancozeb (Fig. 6.7), the epidemics for these treatments did not have the first disease wave observed for all other treatments. Also, since typical waves of black spot could not be distinguished for plants sprayed with $0.25 \times$ and $0.5 \times$ mancozeb, the duration of the waves was assumed to be the same as observed for the other treatments, that is, 8 days. The final amount of disease and the range of disease increase did not differ significantly among these rates of mancozeb.

In spring, only one epidemic wave was observed (Fig. 6.9 and 6.10), which occurred between 8 and 16 days after inoculation. After day 16, the plot of disease proportions transformed to gompits resulted in nearly a straight line. The gompit value was -1.667 at day 8 and -0.529 at day 16 , that is, black spot increased from 0.005 to 0.183 (Table 6.6) during the first epidemic wave. All plants sprayed with fungicides had less 


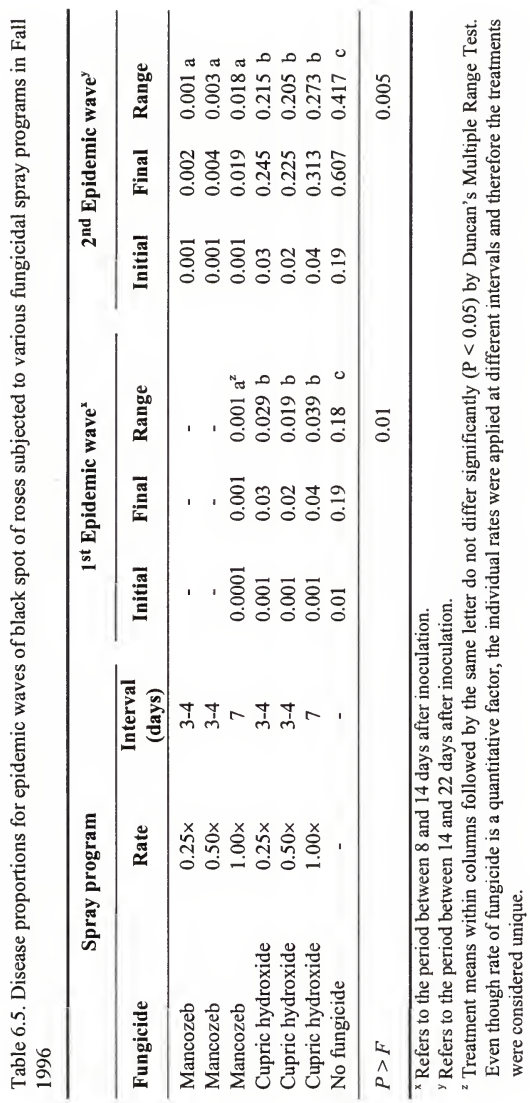




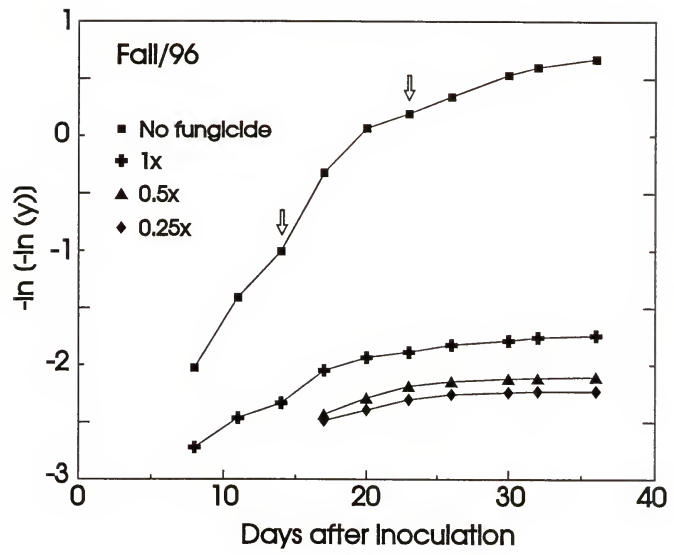

Figure 6.7. Epidemic waves for black spot of roses on plants subjected to weekly (1x rate) or twice-weekly sprays $(0.25 \times$ or $0.5 \times$ rate $)$ of the fungicide mancozeb. Disease proportions $(y)$ were transformed by $-\ln [-\ln (y)]$. Arrows indicate the end of epidemic waves. Experiment developed in Fall 1996. 


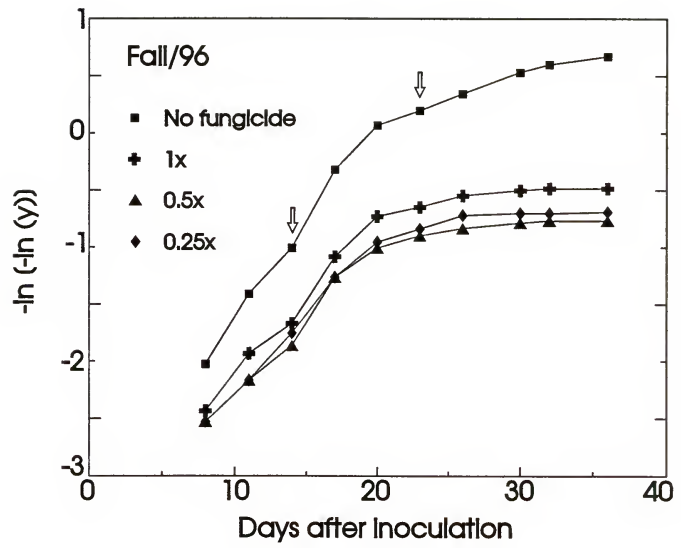

Figure 6.8. Epidemic waves for black spot of roses on plants subjected to weekly (1x rate) or twice-weekly sprays $(0.25 \times$ or $0.5 \times$ rate $)$ of the fungicide cupric hydroxide. Disease proportions $(y)$ were transformed by $-\ln [-\ln (y)]$. Arrows indicate the end of epidemic waves. Experiment developed in Fall 1996. 


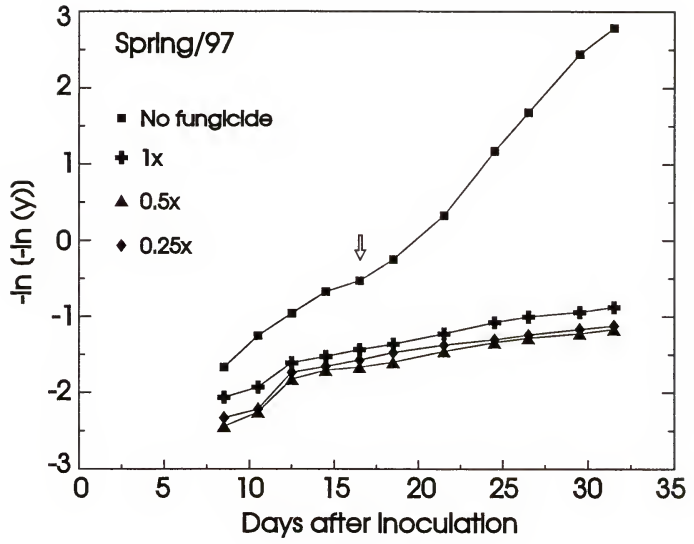

Figure 6.9. Epidemic waves for black spot of roses on plants subjected to weekly (1× rate) or twice-weekly sprays $(0.25 \times$ or $0.5 \times$ rate $)$ of the fungicide mancozeb. Disease proportions $(y)$ were transformed by $-\ln [-\ln (y)]$. Arrows indicate the end of epidemic waves. Experiment developed in Spring 1997. 


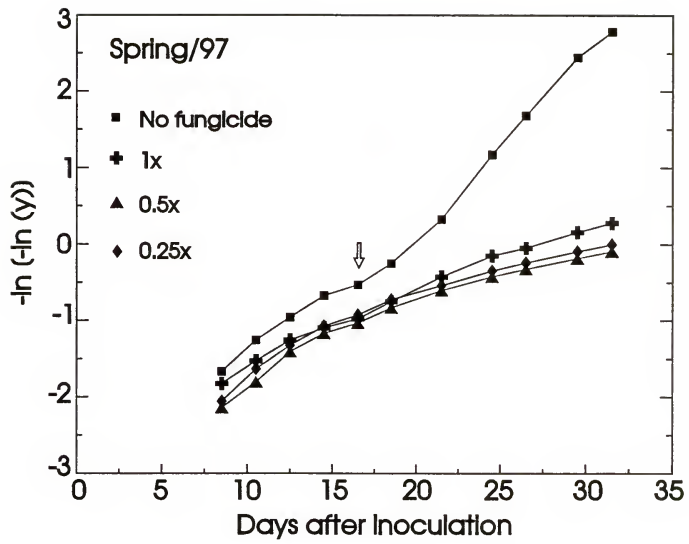

Figure 6.10. Epidemic waves for black spot of roses on plants subjected to weekly (1× rate) or twice-weekly sprays $(0.25 \times$ or $0.5 \times$ rate $)$ of the fungicide cupric hydroxide. Disease proportions $(y)$ were transformed by $-\ln [-\ln (y)]$. Arrows indicate the end of epidemic waves. Experiment developed in Spring 1997. 
increase in disease than the nonsprayed control. Among rates within fungicides, however, the differences on disease intensity were not statistically significant.

Table 6.6. Disease proportions for epidemic waves of black spot of roses subjected to various fungicidal spray programs in Spring 1997

\begin{tabular}{|c|c|c|c|c|c|c|}
\hline \multirow{2}{*}{\multicolumn{3}{|c|}{ Spray program }} & \multicolumn{4}{|c|}{ Disease proportion } \\
\hline & & & \multicolumn{4}{|c|}{ First epidemic wave ${ }^{y}$} \\
\hline Fungicide & Rate & $\begin{array}{c}\text { Interval } \\
\text { (days) }\end{array}$ & Initial & Final & Range & \\
\hline Mancozeb & $0.25 x$ & $3-4$ & 0.0001 & 0.008 & $0.008 \mathrm{a}$ & \\
\hline Mancozeb & $0.50 x$ & $3-4$ & 0.0001 & 0.005 & $0.005 a$ & \\
\hline Mancozeb & $1.00 x$ & 7 & 0.0004 & 0.015 & $0.015 \mathrm{a}$ & \\
\hline Cupric hydroxide & $0.25 x$ & $3-4$ & 0.0004 & 0.08 & 0.08 & $\mathrm{~b}$ \\
\hline Cupric hydroxide & $0.50 x$ & $3-4$ & 0.0002 & 0.06 & 0.06 & $\mathrm{~b}$ \\
\hline Cupric hydroxide & $1.00 x$ & 7 & 0.002 & 0.07 & 0.068 & $\mathrm{~b}$ \\
\hline No fungicide & - & - & 0.005 & 0.183 & 0.178 & c \\
\hline$P>F$ & & & & & 0.002 & \\
\hline
\end{tabular}

${ }^{y}$ Refers to the period between 10 and 18 days after inoculation.

${ }^{2}$ Treatment means within columns followed by the same letter do not differ significantly $(P<$ 0.05 ) by Duncan's Multiple Range Test. Even though rate of fungicide is a quantitative factor, the individual rates were applied at different intervals and therefore the treatments were considered unique.

\section{Effects of Fungicidal Spray Programs on the Disease Progress Curve and AUDPC}

The epidemic curves for black spot on nonsprayed plants were fit to the logistic and Gompertz models by nonlinear regression analysis. The sum of squares for residuals (SSR) that resulted from using the Gompertz equation were lower than those from logistic, thus the Gompertz equation was considered the better fitting model. Also, patterns of residuals (not shown) were commonly observed when residuals of logistic estimates were plotted over time. Therefore, the Gompertz model was chosen to fit the epidemic curves for all treatments. The nonlinear model parameters generated for each epidemic in each experiment are listed in Appendix B. The original disease values and the fitted epidemic curves for the various spray programs are shown in Figures 6.11-6.17. 
In summer, the disease progress curves on plants sprayed with mancozeb at $1 \times$ or $0.25 \times$ rates (Fig. 6.11) were very similar up to 28 days after inoculation. After this point, black spot increased faster on plants sprayed with the $1 \times$ rate and the final disease was nearly twice that observed with the reduced rate. The standardized AUDPC (Table 6.7) for $1 \times$ mancozeb was 0.119 , which was significantly greater $(P=0.05)$ than that calculated for the $0.25 \times$ rate $(0.071)$.

Although the epidemic curves for black spot on plants treated with cupric hydroxide (Fig. 6.12) at $1 \times$ and $0.25 \times$ rates had similar initial and final disease severities, the AUDPC was significantly different $(P=0.05)$ (Table 6.7). At the $1 \times$ rate, black spot developed faster early in the epidemic and this early, severe disease resulted in a greater AUDPC. In the $0.25 \times$-rate treatment, the epidemic was slow to day 30 but fast after that.

Table 6.7. Area under the disease progress curve (AUDPC) for black spot of roses under various programs of fungicidal sprays and different growing seasons in 1996-97

\begin{tabular}{|c|c|c|c|c|c|}
\hline \multicolumn{3}{|c|}{ Spray program } & \multicolumn{3}{|c|}{ AUDPC $^{\mathrm{x}}$} \\
\hline Fungicide & Rate & $\begin{array}{c}\text { Interval } \\
\text { (days) }\end{array}$ & Summer/96 & Fall/96 & Spring/97 \\
\hline Mancozeb & $0.25 x$ & $3-4$ & $0.071 \mathrm{a}^{y}$ & $0.002 \mathrm{a}$ & $0.018 \mathrm{a}$ \\
\hline Mancozeb & $0.50 x$ & $3-4$ & $-z$ & $0.003 \mathrm{a}$ & $0.014 \mathrm{a}$ \\
\hline Mancozeb & $1.00 \times$ & 7 & $0.119 \mathrm{~b}$ & $0.016 \mathrm{a}$ & $0.034 \mathrm{a}$ \\
\hline Manc. + Cup.hyd. & $0.25 x+0.25 x$ & $3-4$ & $0.146 \mathrm{~b}$ & - & - \\
\hline Manc. + Cup.hyd. & $0.25 x+0.25 x$ & 7 & $0.125 \mathrm{~b}$ & - & - \\
\hline Cupric hydroxide & $0.25 x$ & $3-4$ & $0.18 \quad \mathrm{c}$ & $0.178 \mathrm{~b}$ & $0.161 \mathrm{~b}$ \\
\hline Cupric hydroxide & $0.50 x$ & $3-4$ & - & $0.161 \mathrm{~b}$ & $0.14 \quad \mathrm{~b}$ \\
\hline Cupric hydroxide & $1.00 \times$ & 7 & $0.264 \mathrm{~d}$ & $0.229 \mathrm{~b}$ & $0.194 \mathrm{~b}$ \\
\hline No fungicide & - & - & 0.536 & $0.471 \mathrm{c}$ & $0.441 \quad \mathrm{c}$ \\
\hline$P>F$ & & & 0.0001 & 0.0001 & 0.001 \\
\hline
\end{tabular}

${ }^{\mathrm{x}}$ The AUDPC was standardized by dividing the AUDPC values by the total time duration of the epidemics (51 days in Summer/96, 29 days in Fall/96, and 23 days in Spring/97)..

${ }^{y}$ Treatment means within columns followed by the same letter do not differ significantly $(P<$ 0.05 ) by Duncan's Multiple Range Test. Even though rate of fungicide is a quantitative factor, the individual rates were applied at different intervals and therefore the treatments were considered unique.

${ }^{\mathrm{z}}$ Fungicidal spray program not tested in this growing season. 


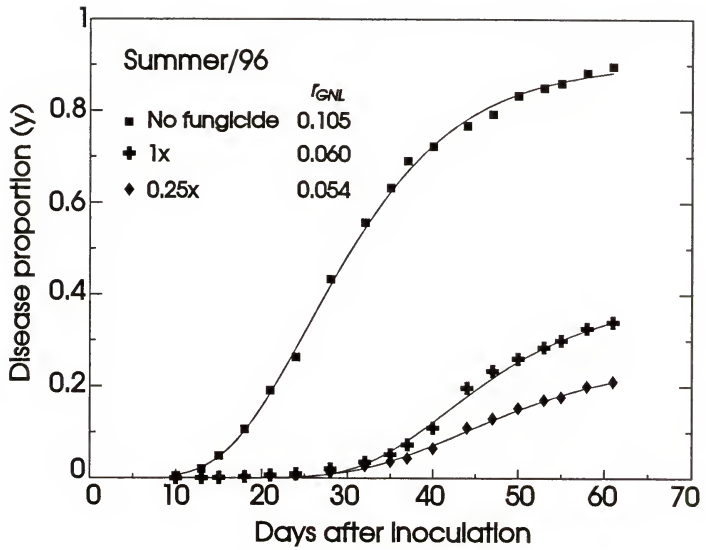

Figure 6.11. Disease progress curves for epidemics of black spot of roses under weekly $(1 \times)$ or twice-weekly sprays $(0.25 \times)$ of fungicide, in which $1 \times$ is the label rate of mancozeb. Epidemic rates $\left(r_{G N L}\right)$ were estimated by nonlinear regression of disease proportions against time using the Gompertz model. Experiment developed in Summer 1996. 


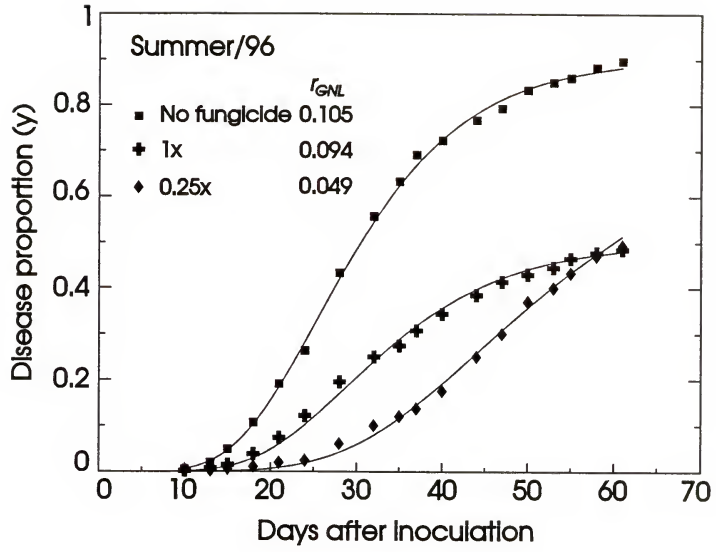

Figure 6.12. Disease progress curves for epidemics of black spot of roses under weekly $(1 \times)$ or twice-weekly sprays $(0.25 \times)$ of fungicide, in which $1 \times$ is the label rate of cupric hydroxide. Epidemic rates $\left(r_{G N L}\right)$ were estimated by nonlinear regression of disease proportions against time using the Gompertz model. Experiment developed in Summer 1996. 
The epidemics for the mixtures of mancozeb plus cupric hydroxide (Fig. 6.13), each at $0.25 \times$ (once a week) or each at $0.1 \times$ (twice a week), had similar development and AUDPCs (Table 6.7). Semi-weekly sprays of mancozeb plus cupric hydroxide, each at $0.1 \times$, resulted in less total disease (AUDPC $=0.146$ ) than twice-weekly applications of cupric hydroxide alone at $0.25 \times$ rate $(\mathrm{AUDPC}=0.18)$, and more disease than twiceweekly sprays of mancozeb alone at $0.25 \times$ rate $($ AUDPC $=0.071)$.

In the fall experiment, although the epidemic of black spot on nonsprayed plants was very fast $\left(0.096\right.$ gompits day $\left.{ }^{-1}\right)$, the disease control provided by sprays of mancozeb was excellent (Fig. 6.14). For example, the AUDPC for black spot under semi-weekly sprays of $0.25 \times$ mancozeb was as low as 0.05 (Table 6.7). Because of such low AUDPC values, significant differences among the three fungicidal rates could not be detected.

The epidemics on plants treated with different rates of cupric hydroxide had similar epidemic rates and disease progress curves (Fig. 6.15). The progress of the epidemics were parallel and the asymptotes were reached 30 days after inoculation. The epidemics for the three rates of cupric hydroxide had similar AUDPCs (Table 6.7).

In the spring experiment, applications of mancozeb or cupric hydroxide reduced the AUDPC for black spot significantly $(P=0.05)$ (Table 6.7). Once again, mancozeb provided better control of black spot (AUDPC $=0.014$ to 0.034 ) than cupric hydroxide (AUDPC $=0.14$ to 0.194 ). For both mancozeb and cupric hydroxide, no differences in AUDPC were associated with the fungicidal rates applied.

\section{Effect of Black Spot on Flower Production}

Flower production of plants subjected to various programs of fungicidal sprays was determined in the fall and spring experiments. In fall, the average number of flowers 


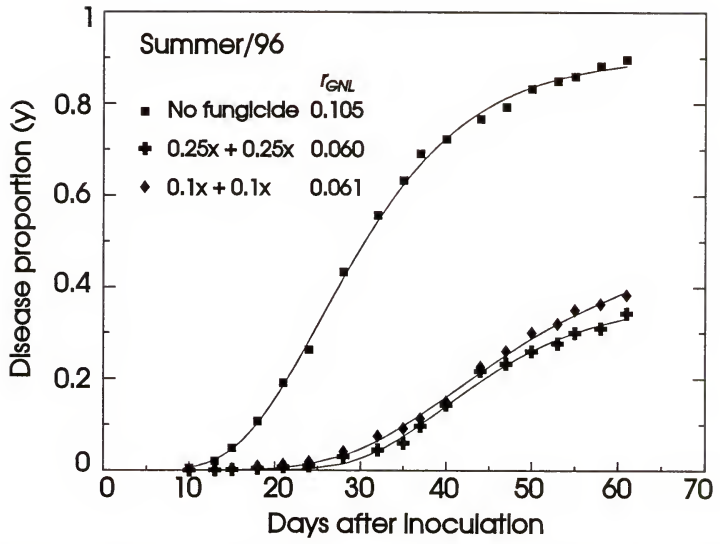

Figure 6.13. Disease progress curves for epidemics of black spot of roses under weekly $(0.25 x+0.25 x)$ or twice-weekly sprays $(0.1 \times+0.1 \times)$ of mancozeb plus cupric hydroxide, in which $1 \times$ is the label rate of each fungicide. Epidemic rates $\left(r_{G N L}\right)$ were estimated by nonlinear regression of disease proportions against time using the Gompertz model. Experiment developed in Summer 1996. 


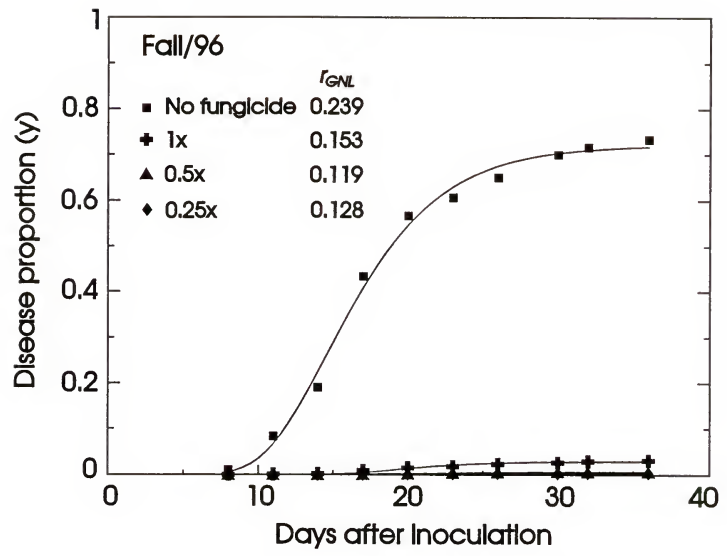

Figure 6.14. Disease progress curves for epidemics of black spot of roses on plants subjected to weekly $(1 \times)$ or twice-weekly sprays $(0.5 \times$ or $0.25 \times)$ of fungicide, in which $1 \times$ is the label rate of mancozeb. Epidemic rates $\left(r_{G N L}\right)$ were estimated by nonlinear regression of disease proportions against time using the Gompertz model. Experiment developed in Fall 1996. 


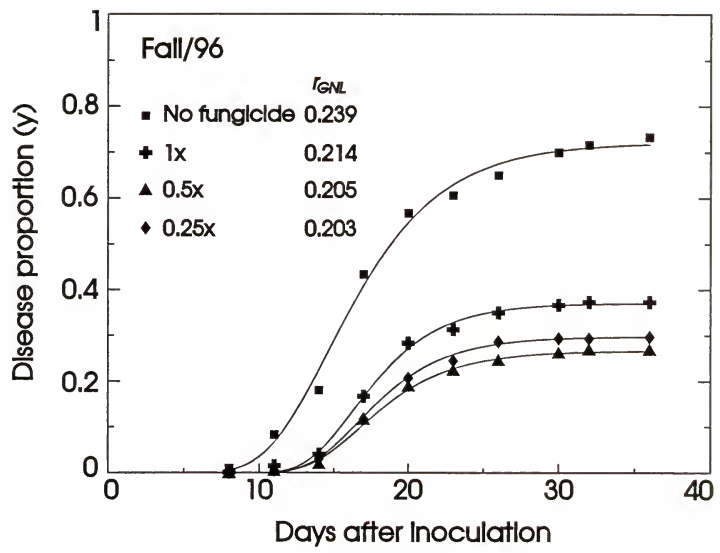

Figure 6.15. Disease progress curves for epidemics of black spot of roses on plants subjected to weekly $(1 \times)$ or twice-weekly sprays $(0.5 \times$ or $0.25 x)$ of fungicide, in which $1 \times$ is the label rate of cupric hydroxide. Epidemic rates $\left(r_{G N L}\right)$ were estimated by nonlinear regression of disease proportions against time using the Gompertz model. Experiment developed in Fall 1996. 


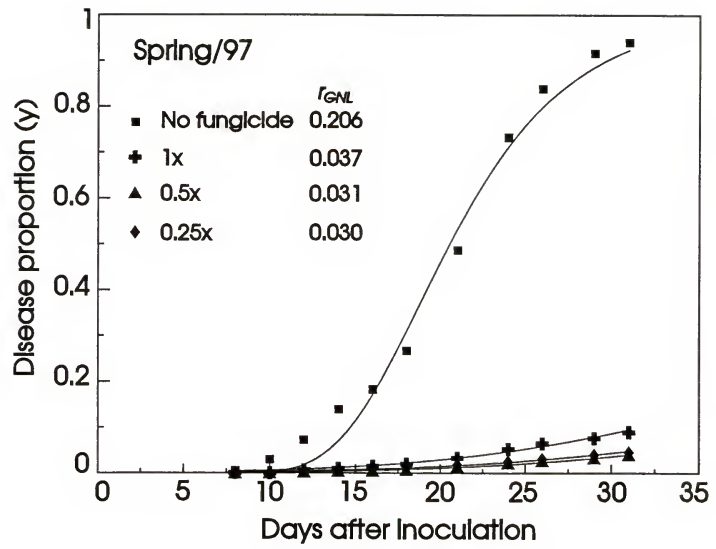

Figure 6.16. Disease progress curves for epidemics of black spot of roses on plants subjected to weekly $(1 \times)$ or twice-weekly sprays $(0.5 \times$ or $0.25 \times)$ of fungicide, in which $1 \times$ is the label rate of mancozeb. Epidemic rates $\left(r_{G N L}\right)$ were estimated by nonlinear regression of disease proportions against time using the Gompertz model. Experiment developed in Spring 1997. 


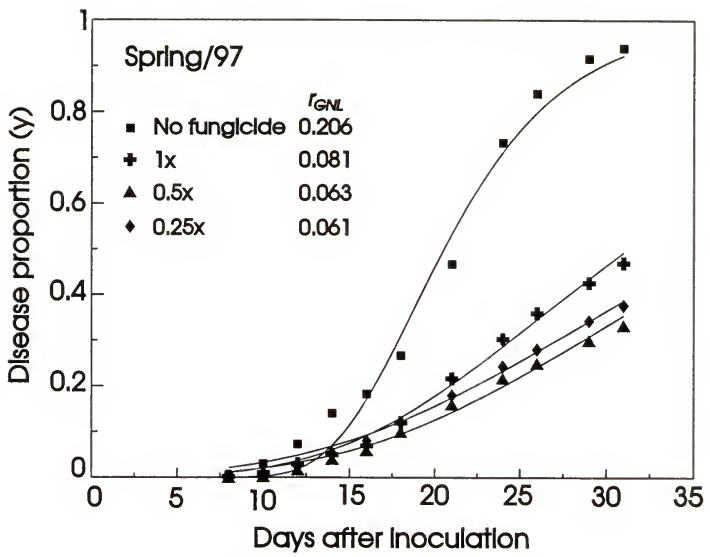

Figure 6.17. Disease progress curves for epidemics of black spot of roses on plants subjected to weekly $(1 \times)$ or twice-weekly sprays $(0.5 \times$ or $0.25 x)$ of fungicide, in which $1 \times$ is the label rate of cupric hydroxide. Epidemic rates $\left(r_{G N L}\right)$ were estimated by nonlinear regression of disease proportions against time using the Gompertz model. Experiment developed in Spring 1997. 


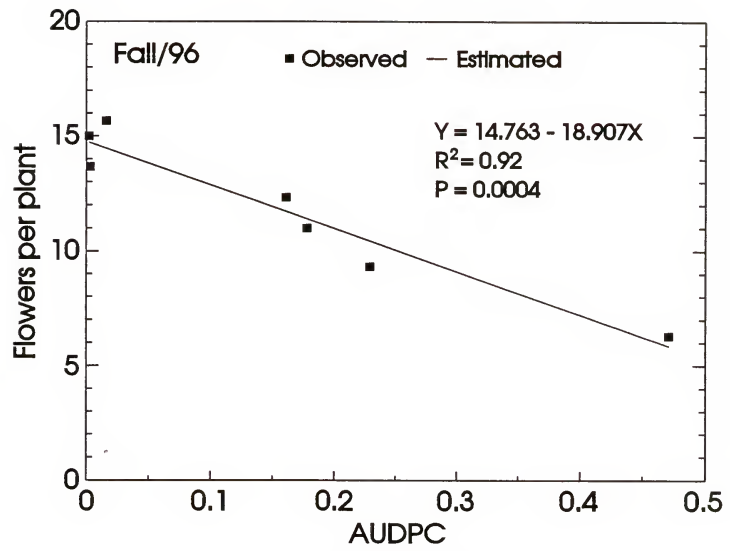

Figure 6.18. Relationship of area under the disease progress curve (AUDPC) for black spot to flower production by rose plants in Fall 1996. 


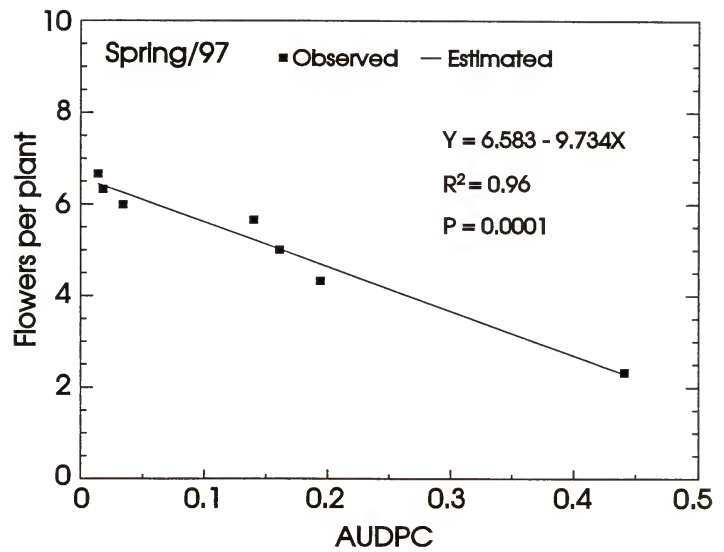

Figure 6.19. Relationship of area under the disease progress curve (AUDPC) for black spot to flower production by rose plants in Spring 1997. 
per plant ranged from 6.3 to 15.7 . Among the rose varieties, the mean production per plant was 9.4 for Oregold, 10.3 for JFK, and 16 flowers for Dolores. The detrimental effect of black spot on flower production (Fig. 6.18) was significant $(P=0.0001)$ and represented by the linear equation $Y=14.763-18.907 X, R^{2}=0.92$, in which $Y$ is the number of flowers per plant and $X$ is the standardized AUDPC.

In spring, the weakened rose plants produced fewer flowers than in the fall experiment. The average number of flowers per plant was 9.6 for Dolores, 4 for JFK, and only 2 for Oregold. Nonsprayed plants of JFK and Dolores yielded no flowers. The linear equation $Y=6.583-9.734 X, R^{2}=0.96$, in which $Y$ is the number of flowers per plant and $X$ is the standardized AUDPC, represented the negative relationship of black spot to flower production (Fig. 6.19).

\section{Discussion}

The wide range of temperatures under which $D$. rosae can infect and colonize rose plants allowed the fungus to cause severe epidemics in all three growing seasons (summer, fall, and spring). Black spot was especially devastating (faster epidemics) in fall and spring because of favorable temperatures, longer dew periods, and more new host tissue. Young rose leaves are markedly more susceptible to black spot than old ones (Horst, 1986). In spring, the progress and spread of black spot was also favored because the initial inoculum of $D$. rosae was applied evenly to the whole plant instead of individual stems as in the summer and fall experiments.

Black spot severely affected all three varieties used in these experiments. Although black spot had latent periods that were 1 or 2 days longer and a rate of lesion expansion that was slower on JFK than on Dolores and Oregold, plants of JFK showed reduced ability to recover (refoliate) from the disease and died at the end of the fall 
experiment. Dolores, despite its high susceptibility to black spot, was able to develop new leaves and produce some flowers. The variety Oregold had also poor development after defoliation, yielded few flowers, and was greatly weakened.

Applications of fungicides reduced the amount of initial disease in all experiments but epidemic onset was delayed only in the fall trial. In summer, fungicides were applied to plants completely foliated. Although plants were scouted for diseased leaves for a period of 10 days, some latent infections possibly remained asymptomatic for longer periods, thus the effects of fungicidal treatments on $y_{0}$ were masked. In spring, some early leaves might have been exposed to the pathogen prior to any spray, so that fungicides had no effect on $t_{0}$. The delay in epidemic onset provided by sprays of the lower rates of mancozeb in fall was probably associated with the protection of the young host tissue at inoculation.

In all three experiments, applications of fungicides reduced the epidemic rate significantly compared to the nonsprayed control. In summer, however, analysis of the epidemic rate alone was not useful to compare fungicides or their rates. For instance, $r_{G N L}$ for mancozeb, at either its full or reduced rate, did not differ from cupric hydroxide, even though the final amount of disease and AUDPC were quite different between the two fungicides. Differences in $r_{G N L}$ among rates of a fungicide were observed in the fall experiment and only for mancozeb. The slower epidemic rates that resulted from applications of $0.25 \times$ and $0.5 \times$ mancozeb to rose plants in fall might have been caused by the delay of epidemic onset provided by the same treatments.

The effects of fungicidal applications on the amount of final disease were greater when the fungicides were applied to plants soon after pruning. For example, $y_{f}$ for $1 \times$ mancozeb varied from 0.34 (summer), in a situation in which fungicides were applied 
initially to plants completely foliated, to 0.03 (fall) in the experiment in which spray treatments were started at the first-bloom stage. These results reinforce the need of early fungicidal applications to achieve successful control of disease. In fall and spring, the final amount of disease was very low and differences were not significant for the various rates of mancozeb.

When gompit values for black spot epidemics were plotted, the disease progress curves had rather flat waves. Short latent periods $(p)$ and long infectious periods $(i)$ (Oort, 1968; Zadoks, 1971), in addition to variable latency (Berger, 1989), are major causes of flattening of epidemic waves. In the fall and spring experiments, $p$ was only 7 to 8 days long. The infectious period for black spot was reported to last from 7 to 10 days (Horst, 1986), but production of conidia associated with lesion expansion may lengthen $i$ considerably. For these reasons, epidemic waves among fungicidal treatments were very difficult to discern and compare for this pathosystem.

The effects of different rates of mancozeb on the epidemic waves varied among experiments. In addition to lowering the height of the epidemic waves, sprays of mancozeb either reduced the initial disease or delayed epidemic onset. Except for the epidemics evaluated in summer, in which the disease progress curves for the $1 \times$ and the $0.25 \times$ rates diverged, black spot had similar disease progress curves, regardless of the rate of fungicide or spray interval used. Therefore, the major effects of the lower fungicidal rates on the epidemic waves were to reduce $y_{0}$ and to delay $t_{0}$.

The low efficiency of cupric hydroxide precluded this fungicide to cause major effects on the epidemic waves. For example, no delay on epidemic onset was observed in any experiment or for any rate of cupric hydroxide. Even when some reduction in initial 
disease was achieved with this compound, it was not enough to provide effective control of black spot in the following weeks.

Black spot caused severe epidemics in all experiments. The fast epidemic rates that resulted from production of new lesions and lesion expansion led to nearly total defoliation of nonsprayed plants. Some susceptible rose varieties even died after being exposed to this disease for three growing seasons. Although black spot was severe, applications of the efficacious fungicide mancozeb provided excellent disease control. In addition to the reduction of the epidemic rate, the preventive applications of mancozeb were able to reduce the amount of initial disease and to decrease the height of the plotted epidemic waves. Lower fungicidal rates were also able to delay epidemic onset in one experiment. No differences in total disease (AUDPC) were attributed to rates of fungicides when sprays were initiated soon after pruning. A delay in the initial fungicidal treatment until after complete leaf expansion resulted in poorer control of disease, especially by the full rate of mancozeb. The fungicide cupric hydroxide was not as efficacious as mancozeb and should not be recommended to control black spot. Mixtures of mancozeb plus cupric hydroxide did not differ from applications of mancozeb alone. 


\section{CHAPTER VII SUMMARY AND CONCLUSIONS}

Fungicides are commonly used to control foliar diseases such as rusts, leaf spots, and downy and powdery mildews. Although three experiments on squash and cucumber could not be used because of lack or low levels of downy and powdery mildews, the additional 18 experiments conducted on two rust and four leaf spot diseases provided a wide variety of situations to study the management of epidemics with low rates of fungicides. Inoculation of plants with the various pathogens, successive plantings in the same fields, and use of infected seeds led to high levels of disease in most experiments. Additionally, the interplot interference (Campbell and Madden, 1990; van der Plank, 1963) caused by the presence of nonsprayed plots and borders, represented an extra pressure of disease for the sprayed plants, which is not common in commercial fields. Therefore, the results obtained in this research provided reliable information on the control of disease by the various fungicidal spray programs tested.

The potential of low rates of fungicides to control disease was demonstrated in both greenhouse and field experiments. In the greenhouse, the low rates protected plants from increased inoculum densities and successive inoculations with rust and leaf-spotting fungi. Also, spray applications at short intervals replaced the fungicide on the leaf surface and kept disease efficiency at $1 / 6$ or less than that measured for the full rate of fungicide. 
In the field, one or two disease waves were observed in the early stages of the epidemics for each pathosystem. Depending upon the latent period of each disease, the epidemic waves were 7 to 14 days long and ended with disease proportions in the range of 0.01 (peanut rust and oat crown rust) to 0.06 (helminthosporium leaf spot and peanut leaf spots), and to 0.21 (black spot of roses). Delay or suppression of the epidemic waves was obtained with applications of fungicides prior to disease establishment. Otherwise, fungicidal treatments only slowed the epidemic rate.

Overall, sprays of the $0.5 \times$ and $0.25 \times$ rates of fungicide, respectively, at average intervals of 6 and 3.5 days, delayed epidemic onset 2 and 6 days, decreased the amount of initial disease 40 and $48 \%$, slowed the epidemic rate 15 and 19\%, reduced the AUDPC 29 and $49 \%$, and increased crop yield 13 and $17 \%$ over applications of the standard (1x) rate at 11-day intervals. Compared to applications of fungicide according to a weather-based schedule (AU-Pnuts), peanut plants sprayed with low rates at frequent intervals averaged $21 \%$ less disease (AUDPC) and $18 \%$ more yield. No significant differences in control of disease and yield on peanuts were observed between low rates of chlorothalonil and the very efficacious systemic fungicide tebuconazole.

Delay of fungicidal applications until action thresholds (disease proportions) in the range of 0.0001 to 0.05 hastened epidemic rates $25 \%$, increased AUDPCs $199 \%$, and reduced crop yields $17 \%$. Additionally, the fast progress of disease in the early epidemic stages and the occurrence of latent infections made the identification of action thresholds very difficult. The use of low rates of fungicide after plants were infected did not improve the control of disease over the standard schedules. On peanuts, the increase in 
AUDPC and the reduction in yield that resulted from late initiation of fungicidal treatments were twice as much under monoculture compared to that after crop rotation.

The application of low fungicidal rates for only short periods early in the season delayed epidemic onset and reduced initial disease but did not keep the epidemic rate slow. The rapid epidemic progress that occurred after sprays of fungicide have ceased markedly increased the amount of disease at the end of the growing season. This problem was prevented by using mixed-rate schedules, which combined early-season sprays of low rates with traditional spray programs used by growers. Mixed-rate schedules improved the control of disease $48 \%$ and increased yield $18 \%$ over regular applications of the standard $(1 \times)$ rate.

Monoculture, the use of diseased seeds, and the presence of infected plant debris were factors that determined early infection of plants and negatively affected the control of peanut leaf spots, helminthosporium leaf spot, and black spot of roses by fungicides. In contrast, sanitation by removal of plant residues, crop rotation, or fallow delayed the occurrence of diseases, allowed the postponement of spray applications, and improved the efficacy of fungicidal treatments. On peanuts, integration of sanitation and sprays of the $0.25 \times$ rate of chlorothalonil resulted in an epidemic delay of 15 days $(9$ days from sanitation and 6 days from early sprays). The rate-reducing resistance of the peanut cultivar Southern Runner allowed the spray intervals of the low rates of fungicide to be lengthened from 3.5 to 7 days $(0.25 \times$ rate $)$ and from 6 to 10 days $(0.5 \times$ rate) without compromising the disease control significantly.

All diseases studied in this research caused severe yield reductions of the host crops. The reduction in yield was higher as plants became infected early in the growing 
season. On peanuts, the cultivar Florunner yielded less than Southern Runner, especially when affected by early epidemics of rust. The relationship of disease to yield was linear for black spot of roses, helminthosporium leaf spot, oat crown rust, and for one epidemic of rust and late leaf spot on peanuts. In most experiments on peanut rust and peanut leaf spots, the diseases reduced crop yield in a nonlinear way. Although integral models that use AUDPC cannot distinguish between early and late occurring epidemics, AUDPC explained $88-96 \%$ of the variation in yield among the various crops. Because the pathosystems included in this investigation were used as disease models to test the potential of low rates of fungicide to manage epidemics, no cost-benefit analyses of the various spray programs were made.

The AUDPC was the most sensitive parameter to compare disease progress curves among the various spray programs. Significant differences in AUDPC occurred in response to variations in epidemic onset $\left(t_{0}\right)$, amount of initial disease $\left(y_{0}\right)$, or epidemic rate $\left(r_{G}\right.$ or $\left.r_{G N L}\right)$. Compared to standard (1x) spray schedules, the AUDPC was $28 \%$ lower when only the epidemic rate was slowed and $43 \%$ lower when $t_{0}$ was delayed or $y_{0}$ was reduced by the low rates of fungicides. The epidemic rate had the least sensitivity $(17 \%)$ to changes in epidemic onset and initial disease. In some epidemics, $r_{G}$ or $r_{G N L}$ was not affected by significant delays in $t_{0}$ or reductions of $y_{0}$. The Gompertz model provided better fitting (lower SSR) of the disease progress curves than the logistic model. The early phase of disease progress curves with typical, high epidemic waves, was not well represented by either the Gompertz or the logistic model. Both models underestimated the real amount of disease. 
In conclusion, the results obtained from this investigation validated the following principles on control of plant diseases by fungicides: $i$ ) preventive applications of low fungicidal rates can delay epidemic onset and reduce the amount of initial disease, ii) delay or suppression of the initial disease waves cause a major reduction of the total epidemic, iii) sprays of fungicide at short intervals maintain enough fungicide on the leaf surface to protect the new plant tissue and to keep infection efficiency low, $i v$ ) application of protectant fungicides after disease establishment has no effect on the epidemic waves, v) the effect of fungicides on delay or suppression of initial epidemic waves for leaf spot diseases can be improved by sanitation or negated by monoculture, $v i$ ) plant varieties with partial resistance to disease may allow the spray interval of low rates of fungicide to be lengthened, vii) inclusion of preventive applications of low fungicidal rates at short spray intervals at the beginning of traditional, calendar-based spray schedules can improve control of disease significantly.

Preventive applications of low rates of fungicide would be especially useful to manage epidemics when the early disease cycles coincide with periods of intense plant growth, when the occurrence of multiple diseases precludes the use of systemic or specific fungicides, where the weather is usually favorable for disease and advisory systems cannot be applied, and for high-value crops such as ornamentals, vegetables, and fruits, whose market value could absorb the cost of extra applications. Because the role of reduced rates of fungicide in the induction of resistance in fungal populations to compounds with specific mode of action is still not clear, the use of low rates should be restricted to broad-spectrum fungicides. 


\section{APPENDIX A \\ SCHEDULES OF FUNGICIDAL SPRAYS}

Table A.1. Spray programs for the fungicide mancozeb evaluated in the 1995 experiments on crown rust of oats

\begin{tabular}{|c|c|}
\hline Spray program & Day of application after inoculation \\
\hline \multicolumn{2}{|c|}{ 1995-A experiment on action thresholds } \\
\hline \multicolumn{2}{|c|}{ Nonsprayed } \\
\hline \multicolumn{2}{|l|}{$0.25 x$} \\
\hline 0 & $0,2,4,7,9,11,14,16,18,21,23,25,28,31,33,35,37,39,42,45,47$, and 49 \\
\hline 0.0001 & $9,11,14,16,18,21,23,25,28,31,33,35,37,39,42,45,47$, and 49 \\
\hline 0.001 & $12,14,16,18,21,23,25,28,31,33,35,37,39,42,45,47$, and 49 \\
\hline 0.01 & $17,19,21,23,25,28,31,33,35,37,39,42,45,47$, and 49 \\
\hline 0.05 & $19,21,23,25,28,31,33,35,37,39,42,45,47$, and 49 \\
\hline \multicolumn{2}{|l|}{$1 \times$} \\
\hline 0 & $0,7,14,21,28,35$, and 42 \\
\hline 0.0001 & $9,16,23,30,37$, and 45 \\
\hline 0.001 & $12,19,26,32,39$, and 45 \\
\hline 0.01 & $17,23,30,37$, and 45 \\
\hline 0.05 & $19,26,32,39$, and 45 \\
\hline \multicolumn{2}{|c|}{ 1995-B experiment on duration of spray programs (days) } \\
\hline \multicolumn{2}{|l|}{ Nonsprayed } \\
\hline \multicolumn{2}{|l|}{$\underline{0.25 x}$} \\
\hline 21 & $0,2,4,7,9,11,14,16,18$, and 21 \\
\hline 28 & $0,2,4,7,9,11,14,16,18,21,23,25$, and 28 \\
\hline 35 & $0,2,4,7,9,11,14,16,18,21,23,25,28,31,33$, and 35 \\
\hline 42 & $0,2,4,7,9,11,14,16,18,21,23,25,28,31,33,35,37,39$, and 42 \\
\hline 49 & $0,2,4,7,9,11,14,16,18,21,23,25,28,31,33,35,37,39,42,45,47$, and 49 \\
\hline \multicolumn{2}{|l|}{$\underline{1 x}$} \\
\hline 21 & $0,7,14$, and 21 \\
\hline 28 & $0,7,14,21$, and 28 \\
\hline 35 & $0,7,14,21,28$, and 35 \\
\hline 42 & $0,7,14,21,28,35$, and 42 \\
\hline 49 & $0,7,14,21,28,35,42$, and 49 \\
\hline
\end{tabular}


Table A.2. Spray programs for the fungicide mancozeb evaluated in the 1996 experiment on crown rust of oats

\begin{tabular}{ll}
\hline Spray program & Day of application after planting \\
\hline Nonsprayed & 109 and 120 \\
Standard $1 \times$ & $100,107,114$, and 121 \\
$0.5 \times$ & $100,104,108,112,116,121$, and 126 \\
$0.25 \times$ & 116,121, and 126 \\
$0.25 \times$-delay & $100,104,108$, and 112 \\
Short I $(0.25 \times)$ & $100,104,108,112,116$, and 121 \\
Short II $(0.25 \times)$ & $100,104,108$, and $112+116$ and 124 \\
Mixed I $(0.25 \times+0.5 \times)$ & $100,104,108$, and $112+116$ \\
Mixed II $(0.25 \times+1 \times)$ & $100,104,108,112,116$, and $121+126$ \\
Mixed III $(0.25 \times+0.5 \times)$ & \\
\hline
\end{tabular}


Table A.3. Spray programs for the fungicide mancozeb evaluated in the 1997 experiment on crown rust of oats

\begin{tabular}{ll}
\hline Spray program & Day of application after inoculation \\
\hline Nonsprayed & 14,25, and 37 \\
Standard $1 \times$ & $0,7,14,21,28$, and 35 \\
$0.5 \times$ & $0,3,7$, and $10+14$ and 25 \\
Mixed I $(0.25 \times+1 \times)$ & $0,3,7,10,14$, and $18+21$ and 33 \\
Mixed II $(.025 \times+1 \times)$ & \\
Action thresholds & \\
$0.25 \times$ & $0,3,7,10,14,18,21,24,28,31,35$, and 37 \\
0 & $10,14,18,21,24,28,31,35$, and 37 \\
0.0001 & $14,18,21,24,28,31,35$, and 37 \\
0.001 & $16,18,21,24,28,31,35$, and 37 \\
0.01 & \\
$1 \times$ & $0,7,14,21,28$, and 35 \\
0 & $10,17,24,31$, and 38 \\
0.0001 & $14,21,28$, and 35 \\
0.001 & $16,23,30$, and 37 \\
0.01 &
\end{tabular}


Table A.4. Spray programs for the fungicide mancozeb evaluated for the control of helminthosporium leaf spot in 1996 and 1997

\begin{tabular}{ll}
\hline Spray program & Day of application after planting \\
\hline$\underline{1996}$ & 91,103, and 114 \\
$1 \underline{x}$ & $84,91,98,106,113$, and 120 \\
$0.5 \underline{x}$ & $84,87,91,94,98,101,106,109,113,117,120$, and 124 \\
$0.25 \underline{x}$ & $91,94,98,101,106,109,113,117,120$, and 124 \\
$0.25 \underline{x}$-delay & $84,87,91$, and 94 \\
Short I $(0.25 \underline{x})$ & $84,87,91,94,98$, and 101 \\
Short II $(0.25 \underline{x})$ & $84,87,91$, and $94+98$ and 120 \\
Mixed I $(0.25 \underline{x}+1 \underline{x})$ & $84,87,91$, and $94+98,106,113$, and 120 \\
Mixed II $(0.25 \underline{x}+0.5 \underline{x})$ & $84,87,91,94,98$, and $101+106,113$, and 120 \\
Mixed III $(0.25 \underline{x}+0.5 \underline{x})$ & \\
1997 & 82,93, and 105 \\
$1 \underline{x}$ & $68,75,82,89,97$, and 105 \\
$0.5 \underline{x}$ & $68,71,75,79,82,85,89,93,97,100,105$, and 109 \\
$0.25 \underline{x}$ & $82,85,89,93,97,100,105$, and 109 \\
$0.25 \underline{x}$-delay & $68,71,75$, and $79+82$ and 93 \\
Mixed I $(0.25 \underline{x}+1 \underline{x})$ & $68,71,75$, and $79+82,89,97$, and 105 \\
Mixed II $(0.25 \underline{x}+0.5 \underline{x})$ & $68,71,75,79,82$, and $85+89,97$, and 105 \\
Mixed III $(0.25 \underline{x}+0.5 \underline{x})$ & \\
\hline
\end{tabular}


Table A.5. Spray programs for the fungicide chlorothalonil evaluated in the 1995 experiment on rust and leaf spots on peanut

\begin{tabular}{ll}
\hline Spray program & Day of application after planting \\
\hline Nonsprayed & $42,56,69$, and 83 \\
Standard $1 \underline{\underline{x}}$ & $42,49,56,63,69,77$, and 83 \\
$0.5 \underline{x}$ & $42,45,49,53,56,60,63,66,69,73,77,80,83$, and 86 \\
$0.25 \underline{x}$ & $42,45,49,53,56,60,63,66,69,73,77,80,83$, and 86 \\
$0.1 \underline{x}$ & $49,63,77$, and 86 \\
$1 \underline{\text {-delay }}$ & $42,52,63,73$, and 83 \\
AU-Pnuts & \\
\hline
\end{tabular}


Table A.6. Spray programs evaluated in the 1996 experiments on rust and leaf spots on peanut

\begin{tabular}{|c|c|}
\hline Spray program & Day of application after planting \\
\hline \multicolumn{2}{|l|}{ Experiment with monoculture } \\
\hline \multicolumn{2}{|l|}{ Nonsprayed } \\
\hline Standard (chlorothalonil) $1 \underline{x}$ & $38,52,66,81$, and 95 \\
\hline 1x-delay & $45,59,72,86$, and 100 \\
\hline $0.5 \underline{x}$ & $38,45,52,59,66,72,81,88$, and 95 \\
\hline $0.5 \underline{x}$-delay & $45,52,59,66,72,81,88$, and 95 \\
\hline $0.25 \underline{x}$ & $\begin{array}{l}38,41,45,48,52,55,59,63,66,69,72,76,81,84,88,91 \text {, } \\
\text { and } 95\end{array}$ \\
\hline $0.25 \underline{x}$-delay & $45,48,52,55,59,63,66,69,72,76,81,84,88,91$, and 95 \\
\hline Mixed $(0.25 \underline{x}+1 \underline{x})$ & $38,41,45,48,52,55,59$, and $63+75$ and 89 \\
\hline AU-Pnuts & $38,48,59,78,89$, and 99 \\
\hline Tebuconazole & $45,59,74$, and 88 \\
\hline \multicolumn{2}{|l|}{ Experiment with crop rotation } \\
\hline \multicolumn{2}{|l|}{ Nonsprayed } \\
\hline Standard $1 \underline{x}$ & $45,59,72,86$, and 99 \\
\hline 1x-delay & $52,66,79,92$, and 105 \\
\hline $0.5 \underline{x}$ & $45,52,59,66,72,81,88$, and 95 \\
\hline $0.5 \underline{x}$-delay & $52,59,66,72,81,88$, and 95 \\
\hline $0.25 \underline{x}$ & $45,48,52,55,59,63,66,69,72,76,81,84,88,91$, and 95 \\
\hline $0.25 \underline{x}$-delay & $52,55,59,63,66,69,72,76,81,84,88,91$, and 95 \\
\hline Mixed $(0.25 \underline{x}+1 \underline{x})$ & $45,48,52,55,59,63$, and $67+81$ and 95 \\
\hline AU-Pnuts & $45,55,77,87,90$, and 100 \\
\hline Tebuconazole & $52,66,81$, and 95 \\
\hline \multicolumn{2}{|c|}{ Experiment with Southern Runner } \\
\hline \multicolumn{2}{|c|}{ Nonsprayed } \\
\hline Standard $1 \underline{x}-14$ days & $38,52,66,81$, and 95 \\
\hline $1 \underline{x}-21$ days & $38,59,81$, and 98 \\
\hline $0.5 \underline{x}$ & $38,48,59,69,81$, and 91 \\
\hline $0.25 \underline{x}$ & $38,45,52,59,66,73,81,88$, and 95 \\
\hline AU-Pnuts & $38,48,63,81$, and 95 \\
\hline
\end{tabular}


Table A.7. Spray programs evaluated in the 1997 experiments on rust and leaf spots on peanut

\begin{tabular}{|c|c|}
\hline Spray program & Day of application after planting \\
\hline \multicolumn{2}{|l|}{ Experiment A } \\
\hline Standard (chlorothalonil) $1 \underline{x}$ & $35,49,63,77$, and 88 \\
\hline $0.5 \underline{x}$ & $35,42,50,57,64,71,79$, and 88 \\
\hline $0.25 \underline{x}$ & $35,38,42,45,49,53,57,60,64,67,71,75,79,84$, and 88 \\
\hline Mixed I $(0.25 \underline{x}+1 \underline{\underline{x}})$ & $35,38,42,45,49$, and $53+57,73$, and 84 \\
\hline Mixed II $(0.25 \underline{\underline{x}}+1 \underline{\underline{x}})$ & $35,38,42,45,49,53,57$, and $60+63,77$, and 88 \\
\hline Mixed III $(0.5 \underline{x}+1 \underline{\underline{x}})$ & 35,42 , and $50+57,73$, and 84 \\
\hline Mixed IV $(0.5 \underline{x}+1 \underline{\underline{x}})$ & $35,42,50$, and $57+63,77$, and 88 \\
\hline AU-Pnuts & $35,45,56,66,77$, and 88 \\
\hline Tebuconazole & $35,50,63$, and 77 \\
\hline \multicolumn{2}{|l|}{ Experiment $B$} \\
\hline \multicolumn{2}{|l|}{ Nonsprayed } \\
\hline Standard (chlorothalonil) $1 \underline{x}$ & $35,49,63$, and 77 \\
\hline $0.5 \underline{x}$ & $35,42,50,57,64$, and 71 \\
\hline $0.25 \underline{x}$ & $35,38,42,45,49,53,57,60,64,67,71$, and 75 \\
\hline Mixed I $(0.25 \underline{x}+1 \underline{x})$ & $35,38,42,45,49$, and $53+57$ and 73 \\
\hline Mixed II $(0.25 \underline{x}+1 \underline{x})$ & $35,38,42,45,49,53,57$, and $60+63$ and 77 \\
\hline Mixed III $(0.5 \underline{x}+1 \underline{x})$ & 35,42 , and $50+57$ and 73 \\
\hline Mixed IV $(0.5 \underline{\underline{x}}+1 \underline{\underline{x}})$ & $35,42,50$, and $57+63$ and 77 \\
\hline AU-Pnuts & $35,45,56,66$, and 77 \\
\hline Tebuconazole & $35,50,63$, and 77 \\
\hline
\end{tabular}


Table A.8. Fungicidal spray programs evaluated for the control of black spot of roses in 1996-97

\begin{tabular}{|c|c|c|}
\hline Fungicide & Rate & Day of application after inoculation \\
\hline \multicolumn{3}{|l|}{ Summer 1996} \\
\hline Nonsprayed & - & \\
\hline Mancozeb & $1 \underline{x}$ & $-1,5,12,19,26,34,41,48$, and 56 \\
\hline Mancozeb & $0.25 \underline{x}$ & $\begin{array}{l}-1,2,5,8,12,16,19,22,26,29,34,37,41,45,48,52 \text {, } \\
\text { and } 56\end{array}$ \\
\hline Cupric hydroxide & $1 \underline{x}$ & $-1,5,12,19,26,34,41,48$, and 56 \\
\hline Cupric hydroxide & $0.25 \underline{x}$ & $\begin{array}{l}-1,2,5,8,12,16,19,22,26,29,34,37,41,45,48,52 \text {, } \\
\text { and } 56\end{array}$ \\
\hline Manc. + Cup. Hyd. & $0.25 \underline{x}+0.25 \underline{x}$ & $-1,5,12,19,26,34,41,48$, and 56 \\
\hline Manc. + Cup. Hyd. & $0.1 \underline{x}+0.1 \underline{x}$ & $\begin{array}{l}-1,2,5,8,12,16,19,22,26,29,34,37,41,45,48,52 \text {, } \\
\text { and } 56\end{array}$ \\
\hline \multicolumn{3}{|l|}{ Fall 1996} \\
\hline Nonsprayed & - & \\
\hline Mancozeb & $1 \underline{x}$ & $-12,-5,1,8,16,23,30,37$ \\
\hline Mancozeb & $0.5 \underline{x}$ & $-12,-9,-5,-2,1,4,8,11,16,20,23,26,30,33$, and 37 \\
\hline Mancozeb & $0.25 \underline{x}$ & $-12,-9,-5,-2,1,4,8,11,16,20,23,26,30,33$, and 37 \\
\hline Cupric hydroxide & $1 \underline{x}$ & $-12,-5,1,8,16,23,30,37$ \\
\hline Cupric hydroxide & $0.5 \underline{x}$ & $-12,-9,-5,-2,1,4,8,11,16,20,23,26,30,33$, and 37 \\
\hline Cupric hydroxide & $0.25 \underline{x}$ & $-12,-9,-5,-2,1,4,8,11,16,20,23,26,30,33$, and 37 \\
\hline \multicolumn{3}{|l|}{ Spring 1997} \\
\hline Nonsprayed & - & \\
\hline Mancozeb & $1 \underline{x}$ & $-20,-13,-6,1,8,15,23$, and 30 \\
\hline Mancozeb & $0.5 \underline{x}$ & $-20,-17,-13,-9,-6,-3,1,5,8,11,15,19,23,26$, and 30 \\
\hline Mancozeb & $0.25 \underline{x}$ & $-20,-17,-13,-9,-6,-3,1,5,8,11,15,19,23,26$, and 30 \\
\hline Cupric hydroxide & $1 \underline{x}$ & $-20,-13,-6,1,8,15,23$, and 30 \\
\hline Cupric hydroxide & $0.5 \underline{x}$ & $-20,-17,-13,-9,-6,-3,1,5,8,11,15,19,23,26$, and 30 \\
\hline Cupric hydroxide & $0.25 \underline{x}$ & $-20,-17,-13,-9,-6,-3,1,5,8,11,15,19,23,26$, and 30 \\
\hline
\end{tabular}




\section{APPENDIX B \\ NONLINEAR REGRESSION PARAMETERS}

Table B.1. Nonlinear regression parameters to fit epidemics of oat crown rust according to the Gompertz model. Experiment on action thresholds conducted in 1995

\begin{tabular}{|c|c|c|c|c|c|c|}
\hline \multirow[b]{2}{*}{ Treatment } & \multicolumn{3}{|c|}{ Model parameter ${ }^{2}$} & \multirow[b]{2}{*}{$R^{2}$} & \multirow[b]{2}{*}{ SSR } & \multirow[b]{2}{*}{$P>F$} \\
\hline & $y_{\max }$ & $B$ & $\boldsymbol{r}_{G N L}$ & & & \\
\hline Nonsprayed & 1.0 & 13.905 & 0.125 & 0.99 & 0.00913 & 0.01 \\
\hline \multicolumn{7}{|l|}{$0.25 \times$ rate } \\
\hline 0.0 & 1.0 & 8.615 & 0.026 & 0.95 & 0.00101 & 0.01 \\
\hline 0.0001 & 0.423 & 7.148 & 0.088 & 0.99 & 0.00134 & 0.01 \\
\hline 0.001 & 0.546 & 6.354 & 0.084 & 0.99 & 0.00258 & 0.01 \\
\hline 0.01 & 0.762 & 6.361 & 0.069 & 0.99 & 0.00293 & 0.01 \\
\hline 0.05 & 0.623 & 8.539 & 0.104 & 0.99 & 0.00328 & 0.01 \\
\hline \multicolumn{7}{|l|}{$1 \times$ rate } \\
\hline 0.0 & 0.459 & 6.114 & 0.058 & 0.99 & 0.00117 & 0.01 \\
\hline 0.0001 & 0.505 & 6.436 & 0.074 & 0.99 & 0.00159 & 0.01 \\
\hline 0.001 & 0.675 & 6.104 & 0.068 & 0.99 & 0.00375 & 0.01 \\
\hline 0.01 & 0.792 & 6.826 & 0.069 & 0.99 & 0.00363 & 0.01 \\
\hline 0.05 & 0.914 & 6.653 & 0.062 & 0.99 & 0.00322 & 0.01 \\
\hline
\end{tabular}

${ }^{z}$ The Gompertz model is $y=y_{\max }\left\{\exp \left[-B \exp \left(-r_{G N L} t\right)\right]\right\}$, in which $y_{\max }=$ proportion of maximum disease, $B=-\ln \left(y_{0}\right)=$ estimated disease at epidemic onset, $r_{G N L}=$ epidemic rate, and $t=$ time in days. 
Table B.2. Nonlinear regression parameters to fit epidemics of oat crown rust according to the Gompertz model. Experiment on duration of spray programs conducted in 1995

\begin{tabular}{|c|c|c|c|c|c|c|}
\hline \multirow[b]{2}{*}{ Treatment } & \multicolumn{3}{|c|}{ Model parameter ${ }^{z}$} & \multirow[b]{2}{*}{$R^{2}$} & \multirow[b]{2}{*}{ SSR } & \multirow[b]{2}{*}{$P>F$} \\
\hline & $y_{\max }$ & $B$ & $r_{G N L}$ & & & \\
\hline Nonsprayed & 1.0 & 10.938 & 0.099 & 0.99 & 0.00614 & 0.01 \\
\hline \multicolumn{7}{|l|}{$\underline{0.25 \times \text { rate }}$} \\
\hline 28 & 1.0 & 37.41 & 0.086 & 0.97 & 0.02083 & 0.01 \\
\hline 35 & 1.0 & 18.555 & 0.058 & 0.98 & 0.00306 & 0.01 \\
\hline 42 & 1.0 & 5.57 & 0.021 & 0.95 & 0.00253 & 0.01 \\
\hline 49 & 0.1 & 14.559 & 0.123 & 0.97 & 0.00021 & 0.01 \\
\hline 56 & 0.212 & 5.482 & 0.038 & 0.99 & 0.00037 & 0.01 \\
\hline \multicolumn{7}{|l|}{$1 \times$ rate } \\
\hline 28 & 1.0 & 19.345 & 0.075 & 0.97 & 0.02485 & 0.01 \\
\hline 35 & 1.0 & 12.076 & 0.054 & 0.98 & 0.00871 & 0.01 \\
\hline 42 & 1.0 & 7.48 & 0.039 & 0.99 & 0.00170 & 0.01 \\
\hline 49 & 1.0 & 5.534 & 0.029 & 0.90 & 0.00260 & 0.01 \\
\hline 56 & 0.33 & 6.07 & 0.062 & 0.98 & 0.00112 & 0.01 \\
\hline
\end{tabular}

${ }^{z}$ The Gompertz model is $y=y_{\max }\left\{\exp \left[-B \exp \left(-r_{G N L} t\right)\right]\right\}$, in which $y_{\max }=$ proportion of maximum disease, $B=-\ln \left(y_{0}\right)=$ estimated disease at epidemic onset, $r_{G N L}=$ epidemic rate, and $t=$ time in days. 
Table B.3. Nonlinear regression parameters to fit epidemics of oat crown rust according to the Gompertz model in 1996

\begin{tabular}{lcccccc}
\hline & \multicolumn{3}{c}{ Model parameter $^{2}$} & & & \\
\cline { 2 - 4 } Treatment & $\boldsymbol{y}_{\max }$ & $\boldsymbol{B}$ & $\boldsymbol{r}_{G N L}$ & $\boldsymbol{R}^{2}$ & SSR & $\boldsymbol{P}>\boldsymbol{F}$ \\
\hline Nonsprayed & 1.0 & 5.04 & 0.048 & 0.99 & 0.00033 & 0.01 \\
Standard 1× & 1.0 & 6.334 & 0.042 & 0.99 & 0.00005 & 0.01 \\
$0.5 \times$ & 1.0 & 6.896 & 0.032 & 0.99 & 0.00001 & 0.01 \\
$0.25 \times$ & 1.0 & 9.315 & 0.059 & 0.99 & 0.00001 & 0.01 \\
$0.25 \times$-delay & 1.0 & 6.048 & 0.042 & 0.99 & 0.00005 & 0.01 \\
\hline
\end{tabular}

${ }^{z}$ The Gompertz model is $y=y_{\max }\left\{\exp \left[-B \exp \left(-r_{G N L} t\right)\right]\right\}$, in which $y_{\max }=$ proportion of maximum disease, $B=-\ln \left(y_{0}\right)=$ estimated disease at epidemic onset, $r_{G N L}=$ epidemic rate, and $t=$ time in days. 
Table B.4. Nonlinear regression parameters to fit epidemics of oat crown rust according to the Gompertz model. Experiment conducted in 1997

\begin{tabular}{lcccccc}
\hline & \multicolumn{3}{c}{ Model parameter $^{2}$} & & & \\
\cline { 2 - 4 } Treatment & $\boldsymbol{y}_{\max }$ & $\boldsymbol{B}$ & $\boldsymbol{r}_{G N L}$ & $\boldsymbol{R}^{2}$ & SSR & $\boldsymbol{P}>\boldsymbol{F}$ \\
\hline Nonsprayed & 1.0 & 20.415 & 0.134 & 0.99 & 0.01432 & 0.01 \\
Standard 1× & 1.0 & 6.986 & 0.052 & 0.99 & 0.00171 & 0.01 \\
$0.5 \times$ & 1.0 & 9.21 & 0.051 & 0.98 & 0.00275 & 0.01 \\
Mixed I & 1.0 & 10.549 & 0.048 & 0.96 & 0.00493 & 0.01 \\
Mixed II & 1.0 & 9.787 & 0.044 & 0.96 & 0.00312 & 0.01 \\
$0.25 \times$ & & & & & & \\
0.0 & 1.0 & 9.586 & 0.042 & 0.98 & 0.00088 & 0.01 \\
0.0001 & 1.0 & 5.995 & 0.05 & 0.99 & 0.00041 & 0.01 \\
0.001 & 1.0 & 6.844 & 0.062 & 0.99 & 0.00055 & 0.01 \\
0.01 & 1.0 & 7.044 & 0.069 & 0.99 & 0.00109 & 0.01 \\
$1 \times$ & & & & & & \\
0.0 & 1.0 & 9.21 & 0.048 & 0.99 & 0.00165 & 0.01 \\
0.0001 & 1.0 & 6.143 & 0.049 & 0.99 & 0.00092 & 0.01 \\
0.001 & 1.0 & 6.525 & 0.058 & 0.99 & 0.00037 & 0.01 \\
0.01 & 1.0 & 7.915 & 0.074 & 0.98 & 0.01017 & 0.01 \\
\hline
\end{tabular}

${ }^{2}$ The Gompertz model is $y=y_{\max }\left\{\exp \left[-B \exp \left(-r_{G N L} t\right)\right]\right\}$, in which $y_{\max }=$ proportion of maximum disease, $B=-\ln \left(y_{0}\right)=$ estimated disease at epidemic onset, $r_{G N L}=$ epidemic rate, and $t=$ time in days. 
Table B.5. Nonlinear regression parameters to fit epidemics of helminthosporium leaf spot on rye according to the Gompertz model

\begin{tabular}{|c|c|c|c|c|c|c|}
\hline \multirow[b]{2}{*}{ Treatment } & \multicolumn{3}{|c|}{ Model parameters $^{2}$} & \multirow[b]{2}{*}{$R^{2}$} & \multirow[b]{2}{*}{ SSR } & \multirow[b]{2}{*}{$P>F$} \\
\hline & $y_{\max }$ & B & $r_{G N L}$ & & & \\
\hline \multicolumn{7}{|l|}{1996} \\
\hline $0.25 x$ & 1.0 & 5.885 & 0.02 & 0.99 & 0.00009 & 0.0001 \\
\hline $0.50 x$ & 1.0 & 6.215 & 0.031 & 0.99 & 0.00014 & 0.0001 \\
\hline $0.25 \times$-delay & 1.0 & 5.56 & 0.034 & 0.98 & 0.00028 & 0.0001 \\
\hline Short I & 1.0 & 6.839 & 0.051 & 0.98 & 0.00067 & 0.0001 \\
\hline Short II & 1.0 & 7.108 & 0.042 & 0.98 & 0.00052 & 0.0001 \\
\hline Mixed I & 1.0 & 6.174 & 0.029 & 0.98 & 0.00021 & 0.0001 \\
\hline Mixed II & 1.0 & 6.237 & 0.025 & 0.98 & 0.00009 & 0.0001 \\
\hline Mixed III & 1.0 & 5.678 & 0.021 & 0.99 & 0.00008 & 0.0001 \\
\hline Standard $1 \times$ & 1.0 & 5.513 & 0.032 & 0.99 & 0.00009 & 0.0001 \\
\hline No fungicide & 1.0 & 5.155 & 0.045 & 0.99 & 0.00057 & 0.0001 \\
\hline \multicolumn{7}{|l|}{1997} \\
\hline $0.25 x$ & 1.0 & 5.998 & 0.027 & 0.99 & 0.00018 & 0.0001 \\
\hline $0.50 x$ & 1.0 & 5.602 & 0.029 & 0.99 & 0.00032 & 0.0001 \\
\hline $0.25 \times$-delay & 1.0 & 4.391 & 0.035 & 0.99 & 0.00042 & 0.0001 \\
\hline Mixed I & 1.0 & 5.862 & 0.033 & 0.99 & 0.00009 & 0.0001 \\
\hline Mixed II & 1.0 & 5.966 & 0.031 & 0.99 & 0.00026 & 0.0001 \\
\hline Mixed III & 1.0 & 6.602 & 0.032 & 0.99 & 0.00036 & 0.0001 \\
\hline Standard $1 \times$ & 1.0 & 5.142 & 0.039 & 0.99 & 0.00046 & 0.0001 \\
\hline No fungicide & 1.0 & 5.688 & 0.064 & 0.99 & 0.00198 & 0.0001 \\
\hline
\end{tabular}

${ }^{2}$ Gompertz model, $y=y_{\max }\left[\exp \left(-\mathrm{B} \exp \left(-r_{G N L} \mathrm{t}\right)\right)\right]$, where $y_{\max }=$ proportion of maximum disease, $\mathrm{B}=-\ln \left(y_{0}\right)=$ estimated disease at the onset of the epidemic, $r_{G N L}=$ epidemic rate, and $t=$ time in days. 
Table B.6. Nonlinear regression parameters to fit mixed epidemics of rust and leaf spots on peanut according to the Gompertz model. Experiment conducted in 1995

\begin{tabular}{lcccccc}
\hline & \multicolumn{9}{c}{ Model parameter $^{2}$} & & & \\
\cline { 2 - 4 } Treatment & $\boldsymbol{y}_{\max }$ & $\boldsymbol{B}$ & $\boldsymbol{r}_{G N L}$ & $\boldsymbol{R}^{2}$ & SSR & $\boldsymbol{P}>\boldsymbol{F}$ \\
\hline Nonsprayed & 1.0 & 12.222 & 0.074 & 0.99 & 0.00289 & 0.01 \\
$0.1 \times$ & 1.0 & 15.697 & 0.044 & 0.99 & 0.00012 & 0.01 \\
$0.25 \times$ & 1.0 & 18.425 & 0.038 & 0.99 & 0.00029 & 0.01 \\
$0.5 \times$ & 1.0 & 15.034 & 0.039 & 0.99 & 0.00011 & 0.01 \\
Standard 1× & 1.0 & 13.783 & 0.039 & 0.99 & 0.00056 & 0.01 \\
1x-delay & 1.0 & 16.243 & 0.056 & 0.99 & 0.00105 & 0.01 \\
AU-Pnuts & 1.0 & 15.053 & 0.039 & 0.99 & 0.00006 & 0.01 \\
\hline
\end{tabular}

${ }^{z}$ The Gompertz model is $y=y_{\max }\left\{\exp \left[-B \exp \left(-r_{G N L} t\right)\right]\right\}$, in which $y_{\max }=$ proportion of maximum disease, $B=-\ln \left(y_{0}\right)=$ estimated disease at epidemic onset, $r_{G N L}=$ epidemic rate, and $t=$ time in days. 
Table B.7. Nonlinear regression parameters to fit mixed epidemics of rust and leaf spots on peanut according to the Gompertz model. Experiment conducted under monoculture of peanuts in 1996

\begin{tabular}{lcccccc}
\hline & \multicolumn{3}{c}{ Model parameter $^{\mathbf{2}}$} & & & \\
\cline { 2 - 4 } Treatment & $\boldsymbol{y}_{\max }$ & $\boldsymbol{B}$ & $\boldsymbol{r}_{G N L}$ & $\boldsymbol{R}^{2}$ & SSR & $\boldsymbol{P}>\boldsymbol{F}$ \\
\hline Nonsprayed & 0.945 & 9.567 & 0.088 & 0.99 & 0.00479 & 0.01 \\
$0.25 \times$ & 0.395 & 7.533 & 0.049 & 0.99 & 0.00103 & 0.01 \\
$0.25 \times$-delay & 0.398 & 6.21 & 0.056 & 0.99 & 0.00281 & 0.01 \\
$0.5 \times$ & 0.428 & 7.903 & 0.051 & 0.99 & 0.00148 & 0.01 \\
$0.5 \times$-delay & 0.451 & 7.21 & 0.056 & 0.99 & 0.00259 & 0.01 \\
Standard 1× & 0.584 & 9.903 & 0.058 & 0.99 & 0.00216 & 0.01 \\
1×-delay & 0.686 & 7.903 & 0.062 & 0.99 & 0.00886 & 0.01 \\
AU-Pnuts & 0.554 & 10.11 & 0.058 & 0.99 & 0.00066 & 0.01 \\
Systemic & 0.541 & 9.21 & 0.058 & 0.99 & 0.00069 & 0.01 \\
Mixed & 0.584 & 10.903 & 0.052 & 0.99 & 0.00357 & 0.01 \\
\hline
\end{tabular}

${ }^{z}$ The Gompertz model is $y=y_{\max }\left\{\exp \left[-B \exp \left(-r_{G N L} t\right)\right]\right\}$, in which $y_{\max }=$ proportion of maximum disease, $B=-\ln \left(y_{0}\right)=$ estimated disease at epidemic onset, $r_{G N L}=$ epidemic rate, and $t=$ time in days. 
Table B.8. Nonlinear regression parameters to fit epidemics of peanut rust according to the Gompertz model. Experiment conducted after fallow in 1996

\begin{tabular}{|c|c|c|c|c|c|c|}
\hline \multirow[b]{2}{*}{ Treatment } & \multicolumn{3}{|c|}{ Model parameter } & \multirow[b]{2}{*}{$R^{2}$} & \multirow[b]{2}{*}{ SSR } & \multirow[b]{2}{*}{$P>F$} \\
\hline & $y_{\max }$ & $\boldsymbol{B}$ & $r_{G N L}$ & & & \\
\hline Nonsprayed & 1.0 & 43.502 & 0.114 & 0.99 & 0.00582 & 0.01 \\
\hline $0.25 x$ & 0.764 & 17.06 & 0.058 & 0.99 & 0.00123 & 0.01 \\
\hline $0.25 x$-delay & 0.784 & 16.561 & 0.059 & 0.99 & 0.00251 & 0.01 \\
\hline $0.5 x$ & 0.877 & 15.458 & 0.061 & 0.99 & 0.00376 & 0.01 \\
\hline $0.5 \times$-delay & 0.879 & 15.009 & 0.062 & 0.99 & 0.00234 & 0.01 \\
\hline Standard $1 \times$ & 0.978 & 17.116 & 0.063 & 0.99 & 0.00208 & 0.01 \\
\hline 1x-delay & 0.976 & 16.431 & 0.071 & 0.99 & 0.00257 & 0.01 \\
\hline AU-Pnuts & 0.927 & 16.25 & 0.064 & 0.99 & 0.00471 & 0.01 \\
\hline Systemic & 0.833 & 14.235 & 0.058 & 0.99 & 0.00191 & 0.01 \\
\hline Mixed & 0.936 & 19.995 & 0.067 & 0.99 & 0.00719 & 0.01 \\
\hline
\end{tabular}

${ }^{z}$ The Gompertz model is $y=y_{\max }\left\{\exp \left[-B \exp \left(-r_{G N L} t\right)\right]\right\}$, in which $y_{\max }=$ proportion of maximum disease, $B=-\ln \left(y_{0}\right)=$ estimated disease at epidemic onset, $r_{G N L}=$ epidemic rate, and $t=$ time in days. 
Table B.9. Nonlinear regression parameters to fit mixed epidemics of rust and leaf spots on peanut according to the Gompertz model. Experiment conducted in 1996 with the cultivar Southern Runner

\begin{tabular}{llccccc}
\hline & \multicolumn{3}{c}{ Model parameter $^{2}$} & & & \\
\cline { 2 - 5 } Treatment & $\boldsymbol{y}_{\max }$ & $\boldsymbol{B}$ & $\boldsymbol{r}_{G N L}$ & $\boldsymbol{R}^{2}$ & SSR & $\boldsymbol{P}>\boldsymbol{F}$ \\
\hline Nonsprayed & 0.702 & 11.502 & 0.077 & 0.99 & 0.00114 & 0.01 \\
$0.25 \times$ & 0.34 & 9.888 & 0.064 & 0.99 & 0.00035 & 0.01 \\
$0.5 \times$ & 0.35 & 9.704 & 0.067 & 0.99 & 0.00031 & 0.01 \\
$1 \times-14$ days & 0.392 & 9.653 & 0.067 & 0.99 & 0.00073 & 0.01 \\
$1 \times-21$ days & 0.502 & 11.439 & 0.072 & 0.99 & 0.00052 & 0.01 \\
AU-Pnuts & 0.38 & 11.242 & 0.068 & 0.99 & 0.00026 & 0.01 \\
\hline
\end{tabular}

${ }^{2}$ The Gompertz model is $y=y_{\max }\left\{\exp \left[-B \exp \left(-r_{G N L} t\right)\right]\right\}$, in which $y_{\max }=$ proportion of maximum disease, $B=-\ln \left(y_{0}\right)=$ estimated disease at epidemic onset, $r_{G N L}=$ epidemic rate, and $t=$ time in days. 
Table B.10. Nonlinear regression parameters to fit mixed epidemics of rust and leaf spots on peanut according to the Gompertz model. Experiment conducted in 1997, in field planted to peanuts the previous year

\begin{tabular}{|c|c|c|c|c|c|c|}
\hline \multirow[b]{2}{*}{ Treatment } & \multicolumn{3}{|c|}{ Model parameter ${ }^{2}$} & \multirow[b]{2}{*}{$R^{2}$} & \multirow[b]{2}{*}{ SSR } & \multirow[b]{2}{*}{$P>F$} \\
\hline & $y_{\max }$ & $B$ & $r_{G N L}$ & & & \\
\hline Nonsprayed & 1.0 & 13.117 & 0.074 & 0.99 & 0.00522 & 0.01 \\
\hline $0.25 x$ & 1.0 & 6.392 & 0.026 & 0.99 & 0.00159 & 0.01 \\
\hline $0.5 x$ & 1.0 & 5.949 & 0.028 & 0.99 & 0.00242 & 0.01 \\
\hline Standard $1 \times$ & 1.0 & 5.363 & 0.031 & 0.98 & 0.00962 & 0.01 \\
\hline Mixed I & 1.0 & 6.768 & 0.029 & 0.99 & 0.00259 & 0.01 \\
\hline Mixed II & 1.0 & 6.085 & 0.026 & 0.99 & 0.00176 & 0.01 \\
\hline Mixed III & 1.0 & 5.886 & 0.028 & 0.98 & 0.00568 & 0.01 \\
\hline Mixed IV & 1.0 & 6.29 & 0.028 & 0.99 & 0.00141 & 0.01 \\
\hline AU-Pnuts & 1.0 & 6.136 & 0.028 & 0.99 & 0.00546 & 0.01 \\
\hline Systemic & 1.0 & 6.53 & 0.025 & 0.99 & 0.00076 & 0.01 \\
\hline
\end{tabular}

${ }^{\mathrm{z}}$ The Gompertz model is $y=y_{\max }\left\{\exp \left[-B \exp \left(-r_{G N L} t\right)\right]\right\}$, in which $y_{\max }=$ proportion of maximum disease, $B=-\ln \left(y_{0}\right)=$ estimated disease at epidemic onset, $r_{G N L}=$ epidemic rate, and $t=$ time in days. 
Table B.11. Nonlinear regression parameters to fit mixed epidemics of rust and leaf spots on peanut according to the Gompertz model. Experiment conducted in 1997, in field planted to peanuts the previous 2 years

\begin{tabular}{lcllccc}
\hline & \multicolumn{3}{c}{ Model parameter $^{2}$} & & & \\
\cline { 2 - 4 } Treatment & $\boldsymbol{y}_{\max }$ & $\boldsymbol{B}$ & $\boldsymbol{r}_{G N L}$ & $\boldsymbol{R}^{2}$ & SSR & $\boldsymbol{P}>\boldsymbol{F}$ \\
\hline Nonsprayed & 1.0 & 6.237 & 0.06 & 0.99 & 0.00273 & 0.01 \\
$0.25 \times$ & 1.0 & 3.57 & 0.014 & 0.94 & 0.00206 & 0.01 \\
$0.5 \times$ & 1.0 & 3.586 & 0.016 & 0.96 & 0.00213 & 0.01 \\
Standard 1× & 1.0 & 3.84 & 0.026 & 0.98 & 0.00211 & 0.01 \\
Mixed I & 1.0 & 3.697 & 0.016 & 0.96 & 0.00182 & 0.01 \\
Mixed II & 1.0 & 3.739 & 0.016 & 0.96 & 0.00184 & 0.01 \\
Mixed III & 1.0 & 3.809 & 0.02 & 0.98 & 0.00166 & 0.01 \\
Mixed IV & 1.0 & 3.498 & 0.015 & 0.94 & 0.00265 & 0.01 \\
AU-Pnuts & 1.0 & 3.84 & 0.021 & 0.98 & 0.00135 & 0.01 \\
Systemic & 1.0 & 3.877 & 0.014 & 0.96 & 0.00092 & 0.01 \\
\hline 2 & &
\end{tabular}

${ }^{z}$ The Gompertz model is $y=y_{\max }\left\{\exp \left[-B \exp \left(-r_{G N L} t\right)\right]\right\}$, in which $y_{\max }=$ proportion of maximum disease, $B=-\ln \left(y_{0}\right)=$ estimated disease at epidemic onset, $r_{G N L}=$ epidemic rate, and $t=$ time in days. 


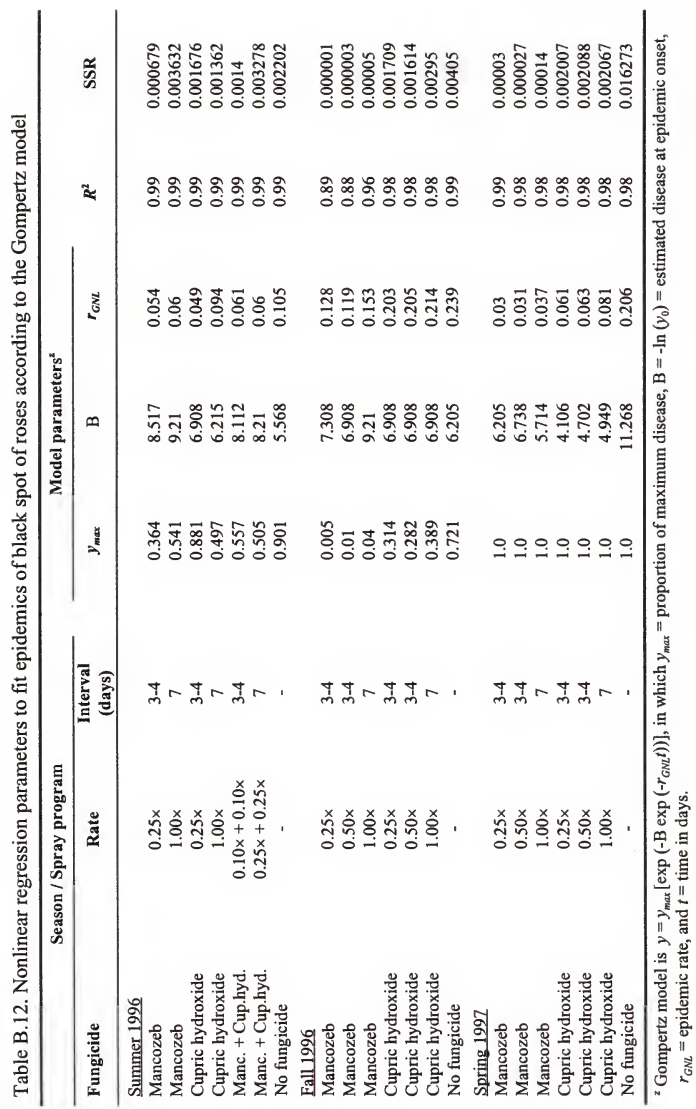


APPENDIX C

WEATHER DATA

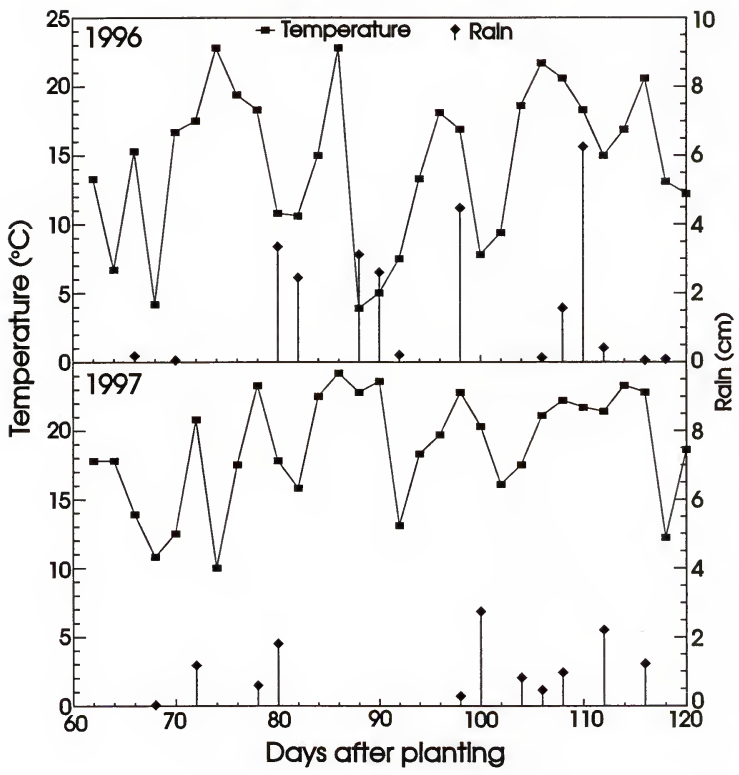

Figure C.1. Average air temperature $\left({ }^{\circ} \mathrm{C}\right)$ and rainfall $(\mathrm{cm})$ during the experiments on Helminthosporium leaf spot on rye in 1996 and 1997. Source: Weather Office, Agronomy Department, IFAS, University of Florida, Gainesville. 


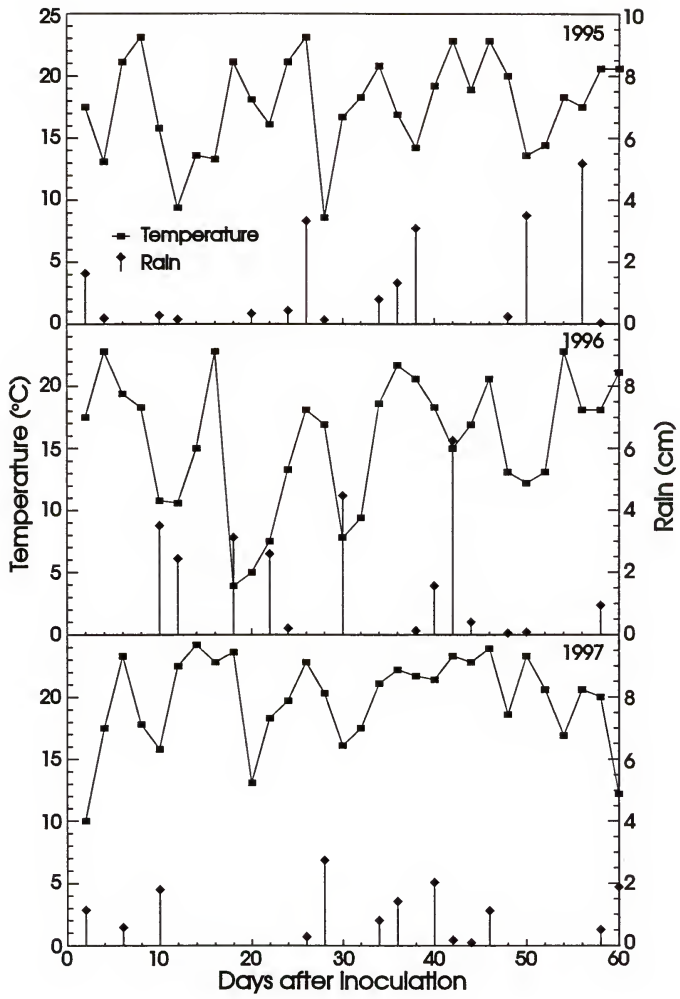

Figure C.2. Average air temperature $\left({ }^{\circ} \mathrm{C}\right)$ and rainfall $(\mathrm{cm})$ during the experiments on crown rust of oats in 1995, 1996, and 1997. Source: Weather Office, Agronomy Department, IFAS, University of Florida, Gainesville. 


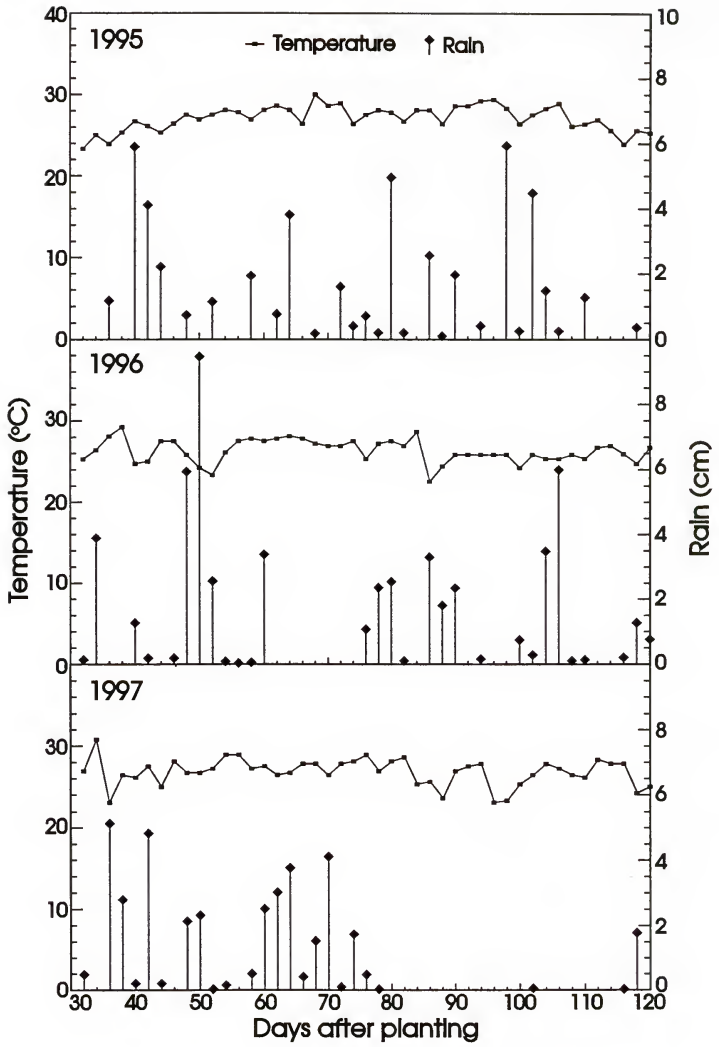

Figure C.3. Average air temperature $\left({ }^{\circ} \mathrm{C}\right)$ and rainfall $(\mathrm{cm})$ during the experiments on peanut rust and peanut leaf spots in 1995, 1996, and 1997. Source: Weather Office, Agronomy Department, IFAS, University of Florida, Gainesville. 


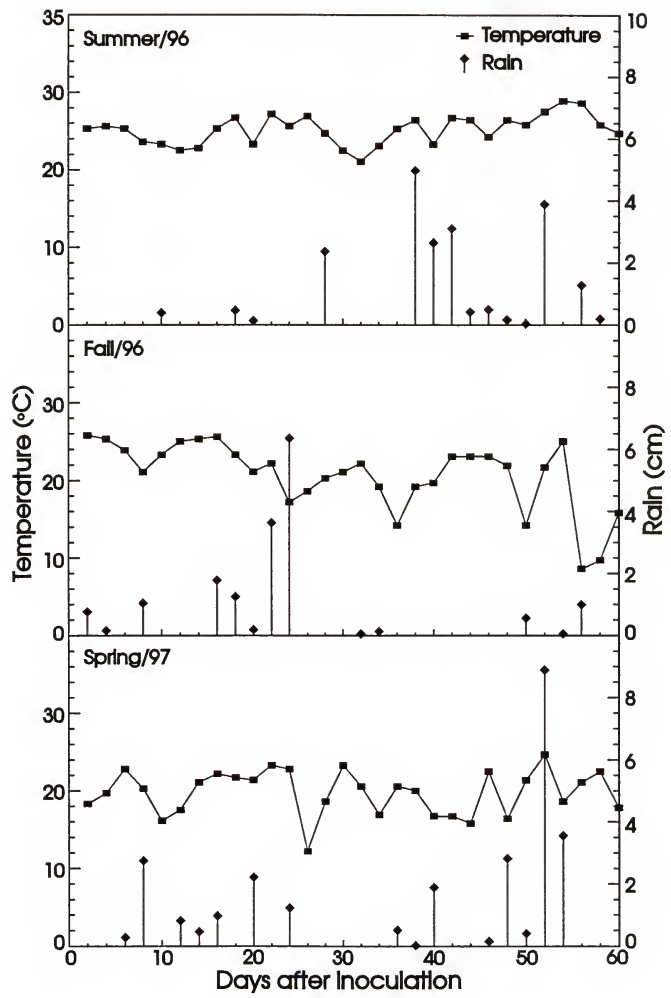

Figure C.4. Average air temperature $\left({ }^{\circ} \mathrm{C}\right)$ and rainfall $(\mathrm{cm})$ during the experiments on black spot of roses in Summer/96, Fall/96, and Spring/97. Source: Weather Office, Agronomy Department, IFAS, University of Florida, Gainesville. 


\section{APPENDIX D \\ COST-BENEFIT ANALYSIS OF FUNGICIDAL SPRAYS FOR PEANUTS}

Table D.1. Estimated costs of applications of the fungicide chlorothalonil according to different rates on peanuts

\begin{tabular}{|c|c|c|c|}
\hline & Fixed & Variable & Total \\
\hline \multicolumn{4}{|l|}{ Cost of application per hour } \\
\hline Tractor $(55 \mathrm{HP})^{1}$ & 6.50 & 3.50 & 10.00 \\
\hline Labor and insurance & & 8.00 & 8.00 \\
\hline Total & & & 18.00 \\
\hline \multicolumn{4}{|l|}{ Cost of application per hectare } \\
\hline $\begin{array}{l}\text { It was assumed that a tractor-mounted sp } \\
\text { four hectares an hour }(18.00 \div 4=4.50)\end{array}$ & brays & & 4.50 \\
\hline \multicolumn{4}{|l|}{ Cost of fungicide per hectare per application ${ }^{2}$} \\
\hline $1 \times$ & & & 21.00 \\
\hline $0.5 x$ & & & 10.50 \\
\hline $0.25 \times$ & & & 5.25 \\
\hline \multicolumn{4}{|l|}{ Total cost per hectare per application } \\
\hline $1 \times$ & & & 25.50 \\
\hline $0.5 x$ & & & 15.00 \\
\hline $0.25 \times$ & & & 9.75 \\
\hline
\end{tabular}

'Source: Hewitt and Shokes, 1996.

${ }^{2}$ Source: Kucharek and Hoover, 1996 (unpublished)

Table D.2. Cost-benefit analysis of applications of the fungicide chlorothalonil on peanuts, cultivar Florunner. Data on number of fungicidal sprays and peanut yield were averaged over four experiments conducted in 1995-97

\begin{tabular}{|c|c|c|c|c|c|c|}
\hline \multirow{2}{*}{$\begin{array}{l}\text { Spray } \\
\text { program }\end{array}$} & \multirow{2}{*}{$\begin{array}{c}\text { Number of } \\
\text { sprays }\end{array}$} & \multicolumn{2}{|c|}{ Cost (U\$/ha) } & \multirow{2}{*}{$\begin{array}{c}\text { Yield } \\
(\mathrm{kg} / \mathrm{ha})\end{array}$} & \multirow{2}{*}{$\begin{array}{c}\text { Additional } \\
\text { revenue } \\
\text { (U\$/ha) }\end{array}$} & \multirow{2}{*}{$\begin{array}{l}\text { Profit } \\
\text { (U\$/ha) }\end{array}$} \\
\hline & & Unity & Total & & & \\
\hline $1 x$ & 4.8 & 25.50 & 122.40 & 2511 & - & - \\
\hline $0.5 x$ & 8.0 & 15.00 & 120.00 & 2861 & 210.00 & 94.00 \\
\hline $0.25 x$ & 15.3 & 9.75 & 149.20 & 3128 & 370.00 & 231.00 \\
\hline
\end{tabular}

${ }^{1}$ Relative to the standard $(1 \times)$ spray program and assuming a peanut price of U $\$ 0.60 / \mathrm{kg}$. 


\section{LIST OF REFERENCES}

Abraham, V., Kushalappa, A. C., Carisse, O., Bourgeois, G., and Auclair, P. 1996. Comparison of decision methods to initiate fungicide applications against Cercospora blight of carrot. Phytoprotection 76:91-99.

Analytis, S. 1973. Methodik der Analyse von Epidemien dargestelt am Apfelschorf (Venturia inaequalis (Cooke) Aderh.). Acta Phytomed. 1:7-75.

Aquino, V. M., Shokes, F. M., Berger, R. D., Gorbet, D. W., and Kucharek, T. A. 1992. Relationships among late leafspot, healthy leaf area duration, canopy reflectance, and pod yield of peanut. Phytopathology 82:546-552.

Backman, P. A., and Crawford, M. A. 1984. Relationship between yield loss and severity of early and late leafspot diseases of peanut. Phytopathology 74:1101-1103.

Bailey, J. E., Johnson, G. L., and Toth, S. J., Jr. 1994. Evolution of a weather-based peanut leaf spot spray advisory in North Carolina. Plant Dis. 78:530-535.

Barreto, M., Suzuki, O. N., Ferreira, M. R., Nakasone, A. K., Scaloppi, E. A. G., and Sanches, A. L. 1996. Persistence of fungicides on peanut leaves (Arachis hypogaea). Fitopatol. Brasil. 21(Suplemento):348 (Abstract).

Bergamin Filho, A., and Amorim, L. 1996. Doenças de Plantas Tropicais: Epidemiologia e Controle Econômico. Ed. Agron. Ceres, São Paulo, SP. 299 pp.

Berger, R. D. 1973. Helminthosporium turcicum lesion numbers related to numbers of trapped spores and fungicide sprays. Phytopathology 63:930-933.

Berger, R. D. 1975a. Rapid disease progress in early epidemic stages. Proc. Am. Phytopathol. Soc. 2:35 (Abstract).

Berger, R. D. 1975b. Predicting the progress of bean rust (Uromyces phaseoli) by average apparent infection rates. Proc. Am. Phytopathol. Soc. 2:73 (Abstract).

Berger, R. D. 1977. Application of epidemiological principles to achieve plant disease control. Ann. Rev. Phytopathol. 15:165-183. 
Berger, R. D. 1980. Measuring disease intensity. In: P. S. Teng and S. V. Krupa (eds.) Proc. E. C. Stakman Commemorative Symposium on Crop Loss Assessment. Univ. of Minnesota Misc. Publ. 7. St. Paul, pp. 28-31.

Berger, R. D. 1981a. Comparison of the Gompertz and logistic equations to describe disease progress. Phytopathology 71:716-719.

Berger, R. D. 1981b. The Gompertz transformation: more appropriate than the logistic to describe disease progress. Phytopathology 71:203 (Abstract).

Berger, R. D. 1988. The analysis of effects of control measures on the development of epidemics. In: J. Kranz and J. Rotem (eds.) Experimental Techniques in Plant Disease Epidemiology. Springer-Verlag. pp 137-151.

Berger, R. D. 1989. Description and application of some general models for plant disease epidemics. In: K. J. Leonard and W. E. Fry (eds.) Plant Disease Epidemiology: Genetics, Resistance, and Management. vol 2. McGraw-Hill. pp 125-149

Berger, R. D., and Luke, H. H. 1979. Spatial and temporal spread of oat crown rust. Phytopathology 69:1199-1201.

Berger, R. D., and Roberts, D. A. 1992. A simple model to describe the peculiar response of latent period to temperature for Uromyces striatus var. medicaginis in alfalfa. Phytopathology 82:1078 (Abstract).

Bissonnette, S. M., D'Arcy, C. J., and Pedersen, W. L. 1994. Yield loss in two spring oat cultivars due to Puccinia coronata f. sp. avenae in the presence or absence of barley yellow dwarf virus. Phytopathology 84:363-371.

Bowen, K. L., Hagan, A. K., Olive, J., and Foster, W. 1994. Application rates and spray intervals of ergosterol-biosynthesis inhibitor fungicides for control of Entomosporium leaf spot of photinia. Plant Dis. 78:578-581.

Bowen, K. L., Young, B., and Behe, B. K. 1995. Management of blackspot of rose in the landscape in Alabama. Plant Dis. 79:250-253.

Brenneman, T. B., and Culbreath, A. K. 1994. Utilizing a sterol demethylation inhibiting fungicide in an advisory program to manage foliar and soilborne pathogens of peanut. Plant Dis. 78:866-872.

Brenneman, T. B., and Murphy, A. P. 1991. Activity of tebuconazole on Cercosporidium personatum, a foliar pathogen of peanut. Plant Dis. 75:699-703.

Brenneman, T. B., Sumner, H. R., and Harrison, G. W. 1990. Deposition and retention of chlorothalonil applied to peanut foliage: Effects of application methods, fungicide formulations, and oil additives. Peanut Sci. 17:80-84. 
Broome, J. C., English, J. T., Marois, J. J., Latorre, B. A., and Aviles, J. C. 1995. Development of an infection model for Botrytis bunch rot of grapes based on wetness duration and temperature. Phytopathology 85:97-102.

Brown, E. M., and Sutton, T. B. 1995. An empirical model for predicting the first symptoms of sooty blotch and flyspeck of apples. Plant Dis. 79:1165-1168.

Bruhn, J. A., and Fry, W. E. 1982. A mathematical model of the spatial and temporal dynamics of chlorothalonil residues on potato foliage. Phytopathology 72:1306-1312.

Campbell, C. L., and Madden, L. V. 1990. Introduction to Plant Disease Epidemiology. Wiley Interscience, NY, $532 \mathrm{pp}$.

Clifford, B. C. 1995. Diseases, pests, and disorders of oats. In: R. W. Welch (ed.) The Oat Crop. Chapman \& Hall, London. pp. 252-278

Cornell, J. A., and Berger, R. D. 1987. Factors that influence the value of the coefficient of determination in simple linear and nonlinear regression models. Phytopathology 77:6370.

Culbreath, A. K., and Brenneman, T. B. 1994. Effect of tank mix combinations of propiconazole and chlorothalonil on late leafspot of peanut. Phytopathology 84:775.

Culbreath, A. K., Brenneman, T. B., and Kvien, C. K. 1992. Use of a resistant peanut cultivar with copper fungicides and reduced fungicide applications for control of late leaf spot. Crop Protection 11:361-365.

Davis, D. P., Jacobi, J. C., and Backman, P. A. 1993. Twenty-four-hour rainfall, a simple environmental variable for predicting peanut leaf spot epidemics. Plant Dis. 77:722-725.

De Waard, M. A., Georgopoulos, S. G., Hollomon, D. W., Ishii, H., Leroux, P., Ragsdale, N. N., and Schwinn, F. J. 1993. Chemical control of plant diseases: problems and prospects. Ann. Rev. Phytopathol. 31:403-421.

Diamond, A. E., Horsfall, J. G., Harberger, J. W., and Stoddard, E. M. 1941. Role of the dosage-response curve in evaluation of fungicides. Conn. Agric. Exp. Stn. Bull. 451.

Drapper, N. R., and Smith, H. 1981. Applied Regression Analysis, $2^{\text {nd }}$ ed. Wiley, New York, 709 pp.

Elliot, V. J., and Spurr Jr, H. W. 1993. Temporal dynamics of chlorothalonil residues on peanut foliage and the influence of weather factors and plant growth. Plant Dis. 77:455460. 
Forcelini, C. A., Reis, E. M., Severo, J. L., and Scheffer, A. C. 1993. Perdas atribuidas à ferrugem da folha da aveia (Puccinia coronata avenae) no Sul do Brasil. Fitopatol. Brasil. 18 (Suplemento):315 (Abstract).

Francl, L. J., Neher, D. A., and Campbell, C. L. 1997. Multiple-point regression models of yield loss. In: L. J. Francl and D. A. Neher (eds.) Exercises in Plant Disease Epidemiology. APS Press, St. Paul, MN. pp 147-151.

Fry, W. E. 1975. Integrated effects of polygenic resistance and a protective fungicide on development of potato late blight. Phytopathology 65:908-911.

Fry, W. E. 1977. Managements with chemicals. In: J. G. Horsfall and E. B. Cowling (eds.) Plant Disease: An Advanced Treatise, vol.1. Academic Press, NY. pp 213-238.

Fry, W. E. 1978. Quantification of general resistence of potato cultivars and fungicide effects for integrated control of potato late blight. Phytopathology 68:1650-1655.

Fry, W. E. 1982. Principles of Plant Disease Management. Academic Press, NY. 378 pp.

Fry, W. E., Bruck, R. I., and Mundt, C. C. 1979. Retardation of potato late blight epidemics by fungicides with erradicant and protectant properties. Plant Dis. Reptr. 63:970-974.

Fulling, B. A., Tigchelaar, E. C., and Latin, R. 1995. Integration of host resistance and weather-based fungicide scheduling for control of anthracnose of tomato fruit. Plant Dis. 79:228-233.

Gilligan, C. A. 1986. Use and misuse of the analysis of variance in plant pathology. In: D. S. Ingram and P. H. Williams (eds.) Advances in Plant Pathology, vol 5. Academic Press, London, NY. pp 225-261.

Gleason, M. L., MacNab, A. A., Pitblado, R. E., Ricker, M. D., East, D. A., and Latin, R. X. 1995. Disease-warning systems for processing tomatoes in Eastern North America: Are we there yet? Plant Dis. 79:113-119.

Gorbet, D. W., Norden, A. J., Shokes, F. M., and Knauft, D. A. 1986. Southern Runner a new leafspot-resistant peanut variety. Univ. of Florida Agric. Expt. Sta, Circ. S-324. 13 pp.

Gorbet, D. W., Shokes, F. M., and Jackson, L. F. 1982. Control of peanut leafspot with a combination of resistance and fungicide treatment. Peanut Science 9:87-90.

Hau, B., and Kranz, J. 1990. Mathematics and statistics for analyses in epidemiology. In: J. Kranz (ed.) Epidemics of Plant Diseases: Mathematical Analysis and Modeling. Springer-Verlag, Berlin. pp 12-52. 
Hewitt, T. D., and Shokes, F. M. 1996. Economics and impacts of decision making in peanut health management. In: H. A. Melouk and F. M. Shokes (eds.) Peanut Health Management. APS Press, St. Paul, MN. Pp 107-113.

Horst, R. K. 1986. Compendium of Rose Diseases. Am. Phytopathol. Soc., St.Paul, MN. $50 \mathrm{pp}$.

Jacobi, J. C., and Backman, P. A. 1991. Evaluation of timing sterol inhibiting fungicides to control peanut diseases. Fungic. Nematicide Tests 46:257.

Jacobi, J. C., and Backman, P. A. 1995. AU-Pnuts Advisory II: Modification of the rulebased leaf spot advisory system for a partially resistant peanut cultivar. Plant Dis. 79:672676.

Jacobi, J. C., Backman, P. A., Davis, D. P., and Brannen, P. M. 1995. AU-Pnuts Advisory I: development of a rule-based system for scheduling peanut leaf spot fungicide applications. Plant Dis. 79:666-671.

Jeger, M. J. 1984. Relation between rate parameters and latent and infectious periods during a plant disease epidemic. Phytopathology 74:1148-1152.

Johnson, C. S., and Beute, M. K. 1986. The role of partial resistance in the management of Cercospora leaf spot of peanut in North Carolina. Phytopathology 76:468-472.

Johnson, D. A., Alldredge, J. R., and Vakoch, D. L. 1996. Potato late blight forecasting models for the semiarid environment of South-Central Washington. Phytopathology 86:480-484.

Johnson, S. B., and Berger, R. D. 1982. On the status of statistics in Phytopathology. Phytopathology 72:1014-1015.

Jong, P. D. de, and Hasper, G. A. 1996. Threshold values for chemical control of powdery mildew (Erysiphe cruciferarum) on brussels sprouts. European Journal of Plant Pathology 102:205-207.

Keinath, A. P. 1995. Fungicide timing for optimum management of gummy stem blight epidemics on watermelon. Plant Dis. 79:354-358.

Ko, W. H., Lin, H., and Kunimoto, R. K. 1975. A simple method for determining efficacy and weatherability of fungicides on foliage. Phytopathology 65:1023-1025.

Kochman, J. K. and Brown, J. F. 1975. Development of the stem and crown rust fungi on leaves, sheaths, and peduncles of oats. Phytopathology 65:1404-1408.

Kranz, J. 1990. Epidemics, their mathematical analysis and modeling: An introduction. In: J. Kranz (ed.) Epidemics of Plant Diseases: Mathematical Analysis and Modeling, $2^{\text {nd }}$ ed. Springer-Verlag, Berlin. pp 1-11. 
Kucharek, T. 1975. Reduction of Cercospora leafspots of peanut with crop rotation. Plant Dis. Reptr. 59:822-823.

Kucharek, T. 1979. Peanut leafspot and rust. Univ. of Florida Coop. Ext. Service, Gainesville, Plant Pathol. Fact Sheet 5. 2 pp.

Kucharek, T. 1988. Diseases of small grains in North and Central Florida. Univ. of Florida Coop. Ext. Service, Gainesville. Plant Pathol. Fact Sheet 38. 6 pp.

Kucharek, T. 1994a. Disease control in oats. In: T. Kucharek and R. Mullin (eds.) 199495 Florida Plant Disease Control Guide, vol. 2. Univ. of Florida Coop. Ext. Service, Gainesville. Special Publication 52. pp. 390-391.

Kucharek. T. 1994b. Disease control in rye. In: T. Kucharek and R. Mullin (eds.) 199495 Florida Plant Disease Control Guide, vol. 2. Univ. of Florida Coop. Ext. Service, Gainesville. Special Publication 52. pp. 406-407.

Kucharek. T. 1994c. Disease control in wheat. In: T. Kucharek and R. Mullin (eds.) 1994-95 Florida Plant Disease Control Guide, vol. 2. Univ. of Florida Coop. Ext. Service, Gainesville. Special Publication 52. pp. 428-433.

Kucharek, T., Cullen, R. E., and Stall, R. E. 1986. Chemical control of foliar diseases of peanuts, peppers, and onions as affected by spray nozzle typles, nozzle orientations, spray intervals, and adjuvants. Plant. Dis. 70:583-586.

Kucharek, T., and Luke, H. H. 1989. Disease control program for wheat. Univ. of Florida Coop. Ext. Service, Gainesville. Ext. Plant Pathol. Rep. 27. 7 pp.

Kucharek, T., and Shokes, F. M. 1995. Disease control program for peanuts. Univ. of Florida Coop. Ext. Service, Gainesville. Ext. Plant Pathol. Rep. 12. 15 pp.

Kucharek, T., Shokes, F. M., and Gobert, D. W. 1991. Considerations for spraying foliar fungicides to control plant diseases as exemplified by studies in Florida from 1968 to 1989 on the control of peanut leafspot. Univ. of Florida Coop. Ext. Service, Gainesville. Bull. 269. 31 pp.

Labrinos, J. L., and Nutter, F. W. 1993. Effects of a protectant versus a systemic fungicide on disease components of peanut late leaf spot. Plant Dis. 77:837-845.

Lacy, M. L. 1994. Influence of wetness periods on infection of celery by Septoria apiicola and use in timing sprays for control. Plant Dis. 78:975-979.

Lacy, M. L., Berger, R. D., Gilbertson, R. L., and Little, E. L. 1996. Current challenges in controlling diseases of celery. Plant Dis. 80:1084-1091.

Large, E. C. 1954. Growth stages in cereals: illustrations of the Feekes scale. Plant Pathol. 3:128-129. 
Leahy, R. M. 1990. Black spot of rose (Rosa sp.). Fla. Dept. Agric. \& Consumer Serv., Gainesville, FL. Circular 327. 2 pp.

Linvill, D. E., and Drye, C. E. 1995. Assessment of peanut leaf spot disease control guidelines using climatological data. Plant Dis. 79:876-879.

Luke, H. H., and Berger, R. D. 1982. Slow rusting in oats compared with the logistic and Gompertz models. Phytopathology 72:400-402.

Madden, L. V. 1980. Quantification of disease progression. Prot. Ecol. 2:159-176.

Madden, L. V. 1986. Statistical analysis and comparison of disease progress curves. In: K. J. Leonard and W. E. Fry (eds.) Plant Disease Epidemiology: Population Dynamics and Management. vol. 1. MacMillan, NY, pp 55-84.

Madden, L. V., and Campbell, C. L. 1990. Nonlinear disease progress curves. In: J. Kranz (ed.) Epidemics of Plant Diseases: Mathematical Analysis and Modeling. Springer-Verlag, Berlin. pp 181-229.

Madden, L. V., Knoke, J. K., and Louie, R. 1982. Considerations for the use of multiple comparison procedures in phytopathological investigations. Phytopathology 72:10151017.

Martinelli, J. A., Reichert, J. L., and Mantese, F. 1984. Evaluation of fungicide efficiency on the control of oat leaf rust (Puccinia coronata). Summa Phytopathologica 10:268-272.

Maytac, C. A., and Bailey, J. E. 1988. Modification of the peanut leaf spot advisory for use on genotypes with partial resistance. Phytopathology 78:640-644.

McFadden, S. E., and Conover, C. A. 1975. Rose culture. Coop. Ext. Service, IFAS, Univ. of Florida, Gainesville. Circular 344-A. 18 pp.

Miller, L. P. 1967. Mechanisms for reaching the site of action. In: D. C. Torgeson (ed.) Fungicides: An Advanced Treatise. Vol, 2. Academic Press, NY. pp 2-59.

Montgomery, D. C. 1991. Design and Analysis of Experiments. $3^{\text {rd }}$ ed. Wiley, NY, 649 pp.

Mudita, I. W., and Kushalappa, A. C. 1993. Ineffectiveness of the first fungicide application at different initial disease incidence levels to manage Septoria blight in celery. Plant Dis. 77:1081-1084.

Neely, D. 1970. Persistence of foliar protective fungicides. Phytopathology 60:15831586.

Neter, J., Wasserman, W., and Kutner, M. H. 1985. Applied Linear Statistical Models. $2^{\text {nd }}$ ed. Irwin, Homewwod, IL, 1127 pp. 
Norden, A. J., Lipscomb, R. W., and Carver, W. A. 1979. Florunner a new peanut variety. Univ. of Florida Circular S-196. 14 pp.

Nutter, F. W., Jr., and Shokes, F. M. 1995. Management of foliar diseases caused by fungi. In: H. A. Melouk and F. M. Shokes (eds.) Peanut Health Management. APS Press, St Paul, MN. pp. 65-73.

Oort, A. J. P. 1968. A model of the early stages of epidemics. Neth. J. Pl. Path. 74:177180.

Pennypacker, S. P., Knoble, H. D., Antle, C. E., and Madden, L. V. 1980. A flexible model for studying plant disease progression. Phytopathology 70:232-235.

Picinini, E. C., and Fernandes, J. M. C. 1989. Effects of fungicides on the control of leaf rust (Puccinia coronata) on oats. Fitopatol. Brasil. 14:343-347.

Plaut, J. L., and Berger, R. D. 1980. Development of Cercosporidium personatum in three peanut canopy layers. Peanut Science 7:46-49.

Politowski, K., and Browning, J. A. 1975. Effect of temperature, light, and dew duration on relative numbers of infections structures of Puccinia coronata avenae. Phytopathology 65:1400-1404.

Porter, D. M., Smith, D. H., and Rodriguez-Kábana, R. 1984. Compendium of Peanut Diseases. The American Phytopathological Society, St. Paul, MN. 73 pp.

Reynolds, K. L., and Neher, D. A. 1997. Statistical comparison of epidemics. In: L. J. Francl and D. A. Neher (eds.) Exercises in Plant Disease Epidemiology. APS Press, St. Paul, MN. pp. 34-37.

Riabov, J. 1978. Effects of spray volume and pressure from a hydraulic boom applicator on severity of peanut leafspot (Cercospora arachidicola, Cercosporidium personatum), peanut yield, and initial spray deposition. M.S. Thesis. Univ. of Florida, Gainesville. 86 pp.

Roelfs, A. P. 1986. Development and impact of regional rust epidemics. In: K. J. Leonard and W. E. Fry (eds.) Plant Disease Epidemiology: Population Dynamics and Management. Vol. 1. Macmillan, NY. pp 129-150.

Sanders, P. L., Houser, W. J., Parish, P. J., and Cole, H. Jr. 1985. Reduced-rate fungicide mixtures to delay fungicide resistance and to control selected turfgrass diseases. Plant Dis. 69:939-943.

Sandler, H. A. 1995. Application of antitranspirant and reduced rate fungicide combinations for fruit rot management in cranberries. Plant Dis. 79:956-961.

SAS Institute, Inc. 1985. SAS/STAT ${ }^{\mathrm{TM}}$ Guide for Personal Computers, Version 6 Edition. SAS Institute Inc., Cary, NC. 378 pp. 
Schein, R. D., Nelson, R. R., Thomas, G. G., Royer, M. H., and Borges, O. 1984. Comparison of the effects of sublethal doses of triadimefon to those of rate-reducing resistance to Erysiphe graminis in wheat. Phytopathology 74:452-456.

Scherm, H., Koike, S. T., Laemmlen, F. F., and van Bruggen, A. H. C. 1995. Field evaluation of fungicide spray advisories against lettuce downy mildew (Bremia lactucae) based on measured or forecast morning leaf wetness. Plant Dis. 79:511-516.

Scherm, H., and Yang, X. B. 1997. Time series analysis. In: L. J. Francl and D. A. Neher (eds.) Exercises in Plant Disease Epidemiology. APS Press, St. Paul, MN. pp. 42-50.

Scientific Programming Enterprises, 1993. Plot IT for Windows, version 3.0. Scientific Programming Enterprises, Haslett, MI. 438 pp.

Severo, J. L., Floss, E. L., and Forcelini, C. A. 1992. Resposta de cultivares de aveia ao control químico da ferrugem da folha. Fitopatol. Brasil. 17:150 (Abstract).

Severo, J. L., Floss, E. L., and Marques, L. 1993. Ensaio de cultivares recomendados de aveia, em Passo Fundo, 1992. In: XIII ${ }^{\text {th }}$ Reunião da comissão sulbrasileira de pesquisa de aveia. Ijui, RS, Brazil. pp 82-86.

Shaner, G. 1991. Fungicide Benefit Assessment: Cereal Crops. National Agricultural Pesticide Impact Assessment Program (NAPIAP), Purdue University, IN. 55 pp.

Shew, B. B., Beute, M. K., Stalker, H. T. 1995. Toward sustainable peanut production: progress in breeding for resistance to foliar and soilborne pathogens of peanut. Plant Dis. 79:1259-1261.

Shokes, F. M., Berger, R. D., Smith, D. H., and Rasp, J. M. 1987. Reliability of disease assessment procedures; a case study with late leafspot of peanut. Oléagineux 42:245-250.

Shokes, F. M., and Gorbet, D. W. 1990. Comparing field performance of leafspotresistant peanut breeding lines. Soil Crop Sci. Soc. Fla. Proc. 49:180-184.

Shtienberg, D. 1995. Rational suppression of sunflower rust: development and evaluation of an action threshold. Plant Dis. 79:506-510.

Simone, G., Kuchareck, T., Elliott, M., and Mullin, R. 1994. 1994-95 Florida Plant Disease Control Guide, vol 1. Univ. of Florida Coop. Ext. Service, Gainesville. Special Publication $52.362 \mathrm{pp}$.

Simons, M. D. 1970. Crown rust of oats and grasses. The American Phytopathological Society, Monograph 5. pp.

Simons, M. D. 1986. Diseases of oats. In: D. A. Lawes and H. Thomas (eds.) Proceedings of the Second International Oats Conference. Martinus Nijhoff Publishers, Dordrecht, The Netherlands. pp. 57-66 
Skylakakis, G. 1983. Theory and strategy of chemical control. Ann. Rev. Phytopathol. 21:117-135.

Smith, D. H., and Littrell, R. H. 1980. Management of peanut foliar diseases with fungicides. Plant Dis. 64:356-361.

Tollenaar, H. 1985. Uredospore germination and development of some cereal rusts from South-Central Chile at constant temperatures. Phytopathologische Zeits. 114:118-125.

van der Plank, J. E. 1963. Plant Diseases: Epidemics and Control. Academic Press, New York. 344 pp.

Van der Plank, J. E. 1967. Epidemiology of fungicidal action. In: D. C. Torgeson (ed.) Fungicides: An Advanced Treatise. Vol. 1. Academic Press, NY. pp 63-92.

van Eeckhout, E., Rush, M. C., and Blackwell, M. 1991. Effects of rate and timing of fungicide applications on incidence and severity of sheath blight and grain yield of rice. Plant Dis. 75:1254-1261.

Waggoner, P. E. 1977. Contributions of mathematical models to epidemiology. Ann. N.Y. Acad. Sci. 287:191-206.

Waggoner, P. E. 1986. Progress curves of foliar diseases: their interpretation and use. In: K. J. Leonard and W. E. Fry (eds.) Plant Disease Epidemiology: Population Dynamics and Management. MacMillan. pp 3-37.

Watson, R. G. 1987. Levels and components of resistance to late leafspot caused by Cercosporidium personatum (Berk. and Curt.) Deighton in the peanut (Arachis hypogaea L.) genotypes Florunner, Southern Runner, and UF81206. Ph.D. Diss., University of Florida, Gainesville. 184 pp.

Wilks, D. S., and Shen, K. W. 1991. Threshold relative humidity duration forecasts for plant disease protection. J. of Appl. Meteorology 30:463-477.

Wu, L., Damicone, J. P., and Jackson, K. E. 1996. Comparison of weather-based advisory programs for managing early leaf spot on runner and Spanish peanut cultivars. Plant Dis. $80: 640-645$.

Yang, X. B., and Zeng, S. M. 1992. Detecting patterns of wheat stripe rust pandemics in time and space. Phytopathology 82:571-576.

Zadoks, J. C. 1971. Systems analysis and the dynamics of epidemics. Phytopathology 61:600-610.

Zadoks, J. C. 1977. On the epidemiological evaluation of fungicide action. Neth. J. Pl. Path. 83(Suppl.):417-426. 
Zadoks, J. C., Chang, T. T., and Konzak, C. F. 1974. A decimal code for the growth stages of cereals. Weed Research 14:415-421.

Zadoks, J. C., and Schein, R. D. 1979. Epidemiology and Plant Disease Management. Oxford Univ. Press, NY. 427 pp. 


\section{BIOGRAPHICAL SKETCH}

Carlos A. Forcelini was born in Passo Fundo, State of Rio Grande do Sul, Brazil, on 28 August 1962. He graduated in Agronomy from the Universidade de Passo Fundo in 1984 and earned a Master of Science degree in Plant Pathology from the Universidade de São Paulo in 1990. Sponsored by the Universidade de Passo Fundo and by the Fundação Capes, Brazil, Carlos Forcelini arrived at the University of Florida in Spring 1994 to pursue a Ph.D. program in Plant Pathology under the supervision of Dr. Richard Berger. For three years in a row, Carlos Forcelini was awarded for outstanding academic achievement by the University of Florida Office for International Students. He also received a first-place award for a paper presented at the student paper competition during the $5^{\text {th }}$ Biennial Meeting of the Florida Phytopathological Society, in 1997. Carlos Forcelini works for the Universidade de Passo Fundo since 1984. He is married to Eveli Forcelini and they have two children, Bruna and Bernardo. 
I certify that I have read this study and that in my opinion it conforms to acceptable standards of scholarly presentation and is fully adequate, in scope and quality, as a dissertation for the degree of Doctor of Philosophy.

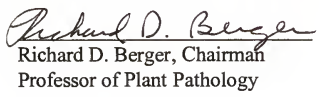

I certify that I have read this study and that in my opinion it conforms to acceptable standards of scholarly presentation and is fully adequate, in scope and quality, as a dissertation for the degree of Doctor of Philosophy.

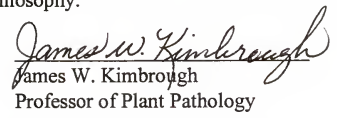

I certify that I have read this study and that in my opinion it conforms to acceptable standards of scholarly presentation and is fully adequate, in scope and quality, as a dissertation for the degree of Doctor of Philosophy.

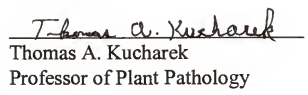

I certify that I have read this study and that in my opinion it conforms to acceptable standards of scholarly presentation and is fully adequate, in scope and quality, as a dissertation for the degree of Doctor of Philosophy.

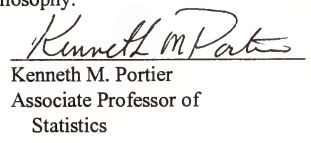

This thesis was submitted to the Graduate Faculty of the College of Agriculture and to the Graduate School and was accepted as partial fulfillment of the requirements for the degree of Doctor of Philosophy.

December, 1997

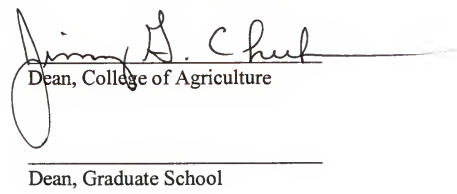

